\title{
Kinetic Studies of Methane-Hydrate Formation from Ice Ih
}

\author{
Dissertation \\ zur Erlangung des Doktorgrades \\ der Mathematisch-Naturwissenschaftlichen Fakultäten \\ der Georg-August-Universiät zu Göttingen
}

vorgelegt von

Doroteya Kancheva Staykova

aus Stara Zagora (BULGARIEN)

Göttingen 2004 
D 7

Referentin/Referent: $\quad$ Prof. Dr. W. F. Kuhs

Korreferentin/Korreferent: Prof. Dr. S. Webb

Tag der mündlichen Prüfung: 20.04.2004 


\section{Abstract}

This thesis work reports on the kinetics of methane hydrate formation from ice Ih powder at conditions of hydrate stability below the quadruple point in order to address open issues on gas hydrate growth. It was conducted in the framework of a DFG-project Ku 920/9 devoted to the study of ice surfaces and ice interfaces.

In this work for the first time results of different experimental methods studying the gas hydrate growth were combined and checked for consistency. Valuable quantitative information of the methane hydrate formation was obtained by neutron diffraction and gas consumption measurements. Similar studies were also attempted on the hydrate growth using X-ray diffraction. An unexpected formation of metastable type II $\mathrm{CO}_{2}$-hydrate together with the ordinary type I was observed by neutron diffraction during the initial growth process. Gas consumption measurements during the methane hydrate growth showed the long-term development reaching an almost full conversion to a hydrate. Scanning electron microscopy provided images of various stages of the transformation of ice to hydrate and guided the development of a phenomenological multistage model of gas hydrate growth from polydisperse ice powder.

For the first time the methane hydrate growth from ice was established as a multistage process of a fast initial coating of the ice surface with a hydrate film (stage I) followed by hydrate growth, limited by reaction at an ice-hydrate interface (stage II), and finally limited by water and gas diffusion through hydrate shells surrounding shrinking ice cores (stage III). For the first time a simulation of methane gas hydrate formation from the beginning to the completion was achieved by means of using the multistage model. The rate constants of the hydrate growth were obtained and used to estimate activation energies of the different stages of the reaction. 


\section{Abstract}

Diese Dissertation berichtet über die Bildungskinetik von Methanhydrat aus pulverförmigem Eis Ih unter Bedingungen der Hydratstabilität unterhalb des Quadrupelpunktes. Sie wurde im Rahmen des DFG-Projektes Ku 920/9 durchgeführt, das sich mit der Untersuchung von Eisgrenzflächen beschäftigte.

Erstmals werden hier Ergebnisse unterschiedlicher experimenteller Methoden zur Erforschung des Gashydratwachstums zusammengeführt und auf Konsistenz überprüft. Quantitative Daten über die Bildung von Methanhydrat wurden durch Neutronendiffraktion und Messungen des Gasverbrauchs gewonnen. Weiterhin wurde versucht, das Hydratwachstum mit Hilfe von Röntgendiffraktion zu untersuchen. Die Anwendung von Neutronenbeugungsverfahren zeigte, dass sich in einem Fall im Anfangsstadium ein metastabiles Typ-II- $\mathrm{CO}_{2}$-Hydrat neben dem üblichen Typ-I-Hydrat bildet. Messungen des Gasverbrauchs während des Wachstumsprozesses zeigten die Neigung zu langen Reaktionsperioden bis zur nahezu vollständigen Umwandlung in Hydrat. Die Rasterelektronenmikroskopie lieferte qualitativ hochwertige Bilder der verschiedenen Transformationsphasen, anhand deren ein phänomenologisches Mehrphasenmodell des Gashydratwachstums von polydispersem Eispulver entwickelt wurde.

So konnte erstmals die Existenz von drei Wachstums-Phasen festgestellt werden: die rasche Bildung eines Hydratfilms über der Eisoberfläche im Anfangsstadium (Phase I), von der Reaktion an der Eis-Hydrat-Grenzfläche gebremstes Hydratwachstum (Phase II) sowie die Diffusion von Wasser und Gas durch die Hydratschicht, von denen die schrumpfenden Eiskerne umgeben sind (Phase III). Zum ersten Mal konnte die Methanhydratbildung vollständig mit Hilfe des Mehrphasenmodells simuliert werden. Die sich daraus ergebenden Reaktionskonstanten des Hydratwachstums wurden zur Einschätzung der Aktivationsenergien der verschiedenen Wachstums-Phasen eingesetzt. 


\section{Contents}

Abstract $\quad$ i

Contents $\quad$ V

1 Introduction 1

1.1 Background...............................................2

1.2 This work ...............................................

References......................................................6

2 Theoretical Section 9

2.1 Thermodynamic Nomenclature................................9

2.1.1 System and Phase Descriptions..........................

2.1.2 Phase Transitions - Basic Concepts.......................11

2.1.3 Driving Force..........................................14

2.1.4 Activation Energy and Nucleation.........................15

2.2 Gas Phase................................................... 18

2.3 Ice Ih Phase.................................................... 19

2.3.1 Bulk Structure and Surface...................................20

2.3.2 Phase Diagram..............................................21

$2.4 \mathrm{CH}_{4}-$ and $\mathrm{CO}_{2}-\mathrm{Gas}$ Hydrate Phases.............................22

2.4.1 Structure..................................................22

2.4.2 Phase Stability............................................24

2.5 Gas Hydrate Formation - Microscopic Conception....................25

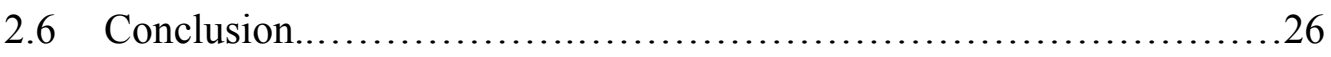

References....................................................28 
3 Experimental Methods 31

3.1 Neutron Powder Diffraction Method.................................31

3.1.1 Neutron Diffraction and Method Description.................31

3.1.2 Rietveld Refinement and GSAS Program......................34

3.1.3 Neutron Diffraction Instrument...............................37

3.1.4 Pressure and Temperature Control.............................39

3.1.5 Ice Powder Starting Material....................................45

3.1.6 Experiments Description.................................47

3.1.7 Data Collection............................................ 49

3.1.8 Data Analyses............................................50

3.2 X-ray Diffraction Method...........................................55

3.2.1 Synchrotron Radiation and Diffraction Set-Up..............55

3.2.2 Long-Time Image Plate Detector Measurements - Problems

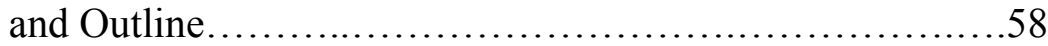

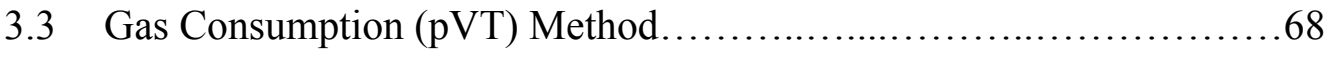

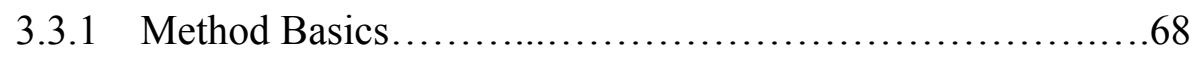

3.3.2 Set-Up and Data Collection..................................68

3.3.3 Sample Recover and Measurement.............................75

3.3.4 Gas-Consumption Data of $\mathrm{CH}_{4}$-clathrate Growth..............76

3.3.5 Data Comparison........................................... 78

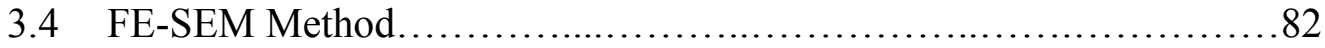

3.4.1 Physics of the Scanning Electron Microscopy ..................82

3.4.2 FE-SEM Instrument - Description and Image Output..........84

3.4.3 Image Data Collection....................................... 88

3.4.4 Starting Material Observations................................... 89

3.4.5 Microstructural Observations................................91

3.4.6 Methane Gas Hydrate Growth - FE-SEM approach............94

$3.5 \quad$ Summary............................................................. 101

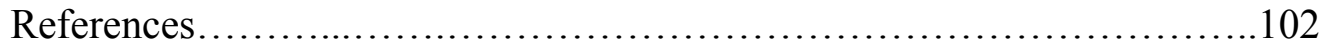


4.1 What is phenomenological modelling?...............................105

4.2 Multistage Model of Gas Hydrate Growth from Ice Powder.............106

4.2.1 Basic Shrinking Ice-Core Model...........................106

4.2.2 Model Extension Including Crack Filling....................108

4.2.3 Analysis of Permeation Resistance of Hydrate Layer........110

4.2.4 Polydisperse Variant of Shrinking Ice-Core Model..........111

4.3 Conclusion................................................................ 114

References......................................................... 116

5 Interpretation of $\mathrm{CH}_{4}$-Gas Hydrate Kinetic Data 119

5.1 Methane Clathrate Formation - Data and Results.....................119

5.1.1 Neutron Diffraction Kinetic Data - Short-term Growth.....119

5.1.2 Gas-Consumption Kinetic Data - Long-term Growth........124

5.1.3 Multistage Model Fit of Data - Results and Discussion.....128

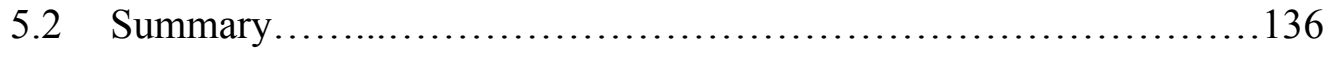

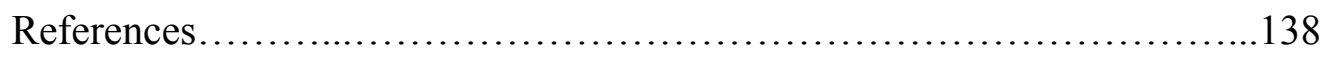

$\begin{array}{ll}\text { Summary } & 139\end{array}$

$\begin{array}{ll}\text { Appendices } & 141\end{array}$

Appendix A.1 ...................................................... 143

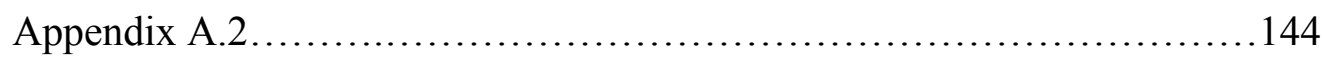

Appendix A.3 .................................................... 145

Appendix A.4 ........................................................ 147

Appendix A.5 ................................................... 150

Appendix B....................................................... 153

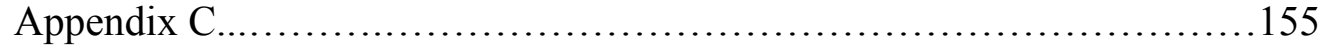

Appendix D.........................................................

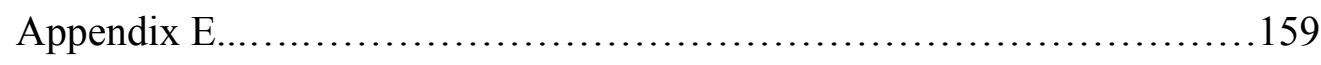

Appendix F...................................................... 161 
Appendix G................................................... 163

Acknowledgements

Lebenslauf 


\section{Chapter 1}

\section{Introduction}

Since their discovery in XIX ${ }^{\text {th }}$ century until now, the gas hydrates are a subject of research with an ever-increasing intensity depending on the people's interest. In the corresponding literature terms of gas hydrates, clathrates, clathrate hydrates, gas clathrate hydrates, solid hydrates and etc. are used to express nonstoichiometric inclusion compounds encaging small guest molecules in a framework of hydrogen bonded water molecules. Likewise, these terms are used as synonyms within the presented work. The phenomena of gas hydrate formation grew from a pure scientific curiosity to an important factor in the gas industry, economy and ecology. Nowadays, for a number of reasons, a challenging topic is the time dependant phenomena of gas hydrate formation and decomposition. For example, if hydrate stability conditions occur within gas and oil transport systems they could be completely blocked. Knowledge of the formation and decomposition kinetics of $\mathrm{CH}_{4}$-gas hydrate would allow for a future extraction of gas from natural gas hydrates, as well as a possible economic transport and storage of gas in the form of hydrate and eventually to clarify the role of methane gas in the climate change. Gas hydrates are also believed to play a role in processes running not only on the Earth but also on some other places in the Solar system. For instance, they are assumed to be involved in some terraforming processes on Mars. Moreover, a methane hydrate existence is suspected on the Saturn's satellite Titan. Consequently, a number of questions concerning the gas hydrate kinetics were posed, like for example:

- How to promote or inhibit the gas hydrate formation?

- How fast is the formation of gas hydrate and how to model and predict it?

- What processes control the gas hydrate formation? 
- What knowledge about the natural gas hydrate formation could be deduced from studies of the growth kinetics of synthetic gas hydrates?

A common feature of all these problems is the necessity of understanding the kinetics of gas hydrate formation, and in particular the one of methane clathrate, with respect to the thermodynamic conditions. It is difficult to obtain such information because the gas hydrate kinetics is a complex multiphase process. Thus, a need of fundamental understanding of the process in a well-defined reacting system appears. Then this knowledge can serve as a reference for further studies of the gas hydrate formation kinetics in more complex systems - for example formation in sediments or systems that include kinetic inhibitors. In order to obtain this knowledge experimental information has to be collected and analysed.

\subsection{Background}

It was recently discovered that some clathrate-hydrate crystals possess a sponge-like porous microstructure. Under certain conditions laboratory-made gas hydrates showed unique porous microstructures on a nanometric scale observed directly by a cryo field-emission scanning electron microscopy (Kuhs et al., 2000; Klapproth, 2002; Klapproth et al., 2003; Staykova et al., 2002, 2003). Similar porous microstructures were found also in the sea-floor gas hydrates (Suess et al., 2002). The sub-micron porous structure, revealed by the gas hydrates, makes them unique crystalline compounds and general physical concepts of their growth are believed to be similar in different situations. In order to constrain better the formation conditions and the time scales for the hydrate formation in geological settings it is necessary to quantify the conditions leading to such microstructures by well-controlled laboratory experiments.

Based on experimental studies of $\mathrm{CO}_{2}$ and fluorocarbon hydrate growth at liquid-liquid interfaces (Aya et al., 1992; Uchida and Kawabata, 1995; Sugaya and Mori, 1996), Mori proposed a porous microstructure of the hydrate layers, intervening the two liquid phases and developed capillary permeation model of water transport across the films during their growth (Mori and Mochizuki, 1997, 2000; Mori, 1998).

The kinetics of gas hydrate formation in aqueous laboratory systems was reviewed by Sloan (1998). It was established that the gas hydrate growth from water, shown by a typical curve (Fig.1), reveals different steps. 


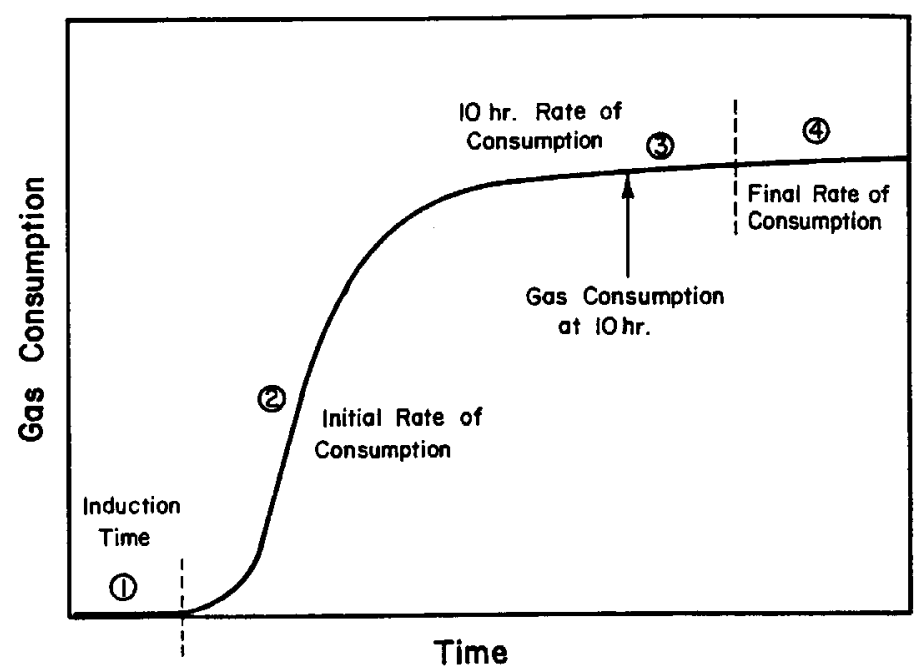

Figure 1. Scheme of the gas hydrate growth from water (Sloan, 1998)

The gas hydrate formation in aqueous systems undergoes an initial induction period (minutes to hours) of formation of critical gas hydrate nuclei. It is followed by a fast initial growth, which is slowing down with time.

Less work was devoted to the formation of gas hydrates from ice. Barrer and Edge (1967) emphasised the role of gas-ice surface area for argon, krypton and xenon gas hydrate formations considered as surface reactions. Regarding ice crystals as initially coated by a gas-impermeable clathrate, the authors performed mechanically shattering of their samples to provide a fresh ice surface promoting the reaction. Likewise, Hwang et al. (1990) studied the methane-hydrate growth on ice as an interfacial (heterogeneous) phenomenon taking place when melting ice is involved and observed an increase of the clathrate formation rates with an increase of pressure. The authors assumed the growth rates were predominantly determined by the rate of mass and heat transfer with respect to the growth surface. Using the technique of Magnetic Resonance Microimaging (MRM), Moudrakovski et al. (2002) observed an occurrence of thin hydrate coverage on the ice surface when studying the reaction between ice particles and $\mathrm{CO}_{2}$. Unfortunately, they could not deduce quantitative information on the formation process from their images. In accordance with experimental observations (Uchida et al., 1992, 1994; Stern et al., 1998; Henning et al., 2000; Takeya et al., 2000; Staykova et al., 2002, 2003), a thin gashydrate film rapidly spread initially over the ice surface. Subsequently, the only possibility to maintain the clathration reaction was the transport of gas molecules through the growing hydrate 
layer to the ice-hydrate interface and transport of water molecules from the ice core to the outer hydrate-gas interface. With the assumption of diffusion-limited clathrate growth the in situ kinetic data of $\mathrm{CO}_{2}$-gas hydrate obtained by laboratory energy dispersive X-ray diffraction (Takeya et al. (2000)) and time-of-flight neutron powder diffraction (Henning et al. (2000, 2001)) were interpreted on the basis of shrinking-core models formulated for a single ice particle. Later, Wang et al. (2002) used the same approach to fit in situ kinetic data of $\mathrm{CH}_{4}$-hydrate formation obtained by a time-of-flight neutron powder diffraction technique. Based on the observations of porous sub-micron structure of gas hydrates (see above), Salamatin and Kuhs, 2002 assumed the gas and water mass transport through the hydrate layer to be much easier. Thus the clathration reaction itself together with the gas and water transfer over the phase boundaries may well become the rate-limiting step(s) at the later stage of hydrate formation that proceeds after the icegrain coating. Subsequently it was expected an onset of a further stage of the hydrate formation controlled by the gas and water diffusion through the clathrate phase, especially when a highly consolidated ice-hydrate structure develops with thick and dense hydrate shells surrounding the ice cores.

\subsection{This work}

This thesis work presents fundamental studies on the kinetics of methane gas hydrate formation starting from ice Ih. The surface of ice appears to play a major role in the hydrate formation in which gas molecules interact in a presently unknown way with the (disordered) water molecules at the ice surface. By studying the reaction kinetics on the ice-gas or ice-hydrate interface it was also hoped to learn more about the ice interface properties, in particular what concerns the mobility of the water molecules. As it was discussed above, this is necessary for the understanding of the process and as a guide to further investigations. It was already established that the nucleation of hydrate formation was very fast starting from ice (Kuhs et al. (1992), Stern et al. (1998)) and considerably slower starting from a liquid (Sloan, 1998). A way to produce a starting sample with a large surface area, promoting the hydrate formation reaction, was found in the preparing of ice powder at physico-chemical laboratory - GZG, Göttingen. Thus, in situ kinetic studies were confined to particular thermodynamic conditions of $\mathrm{CH}_{4}$ - and $\mathrm{CO}_{2}$ - hydrate formation on ice-grains surfaces, under relatively high pressures, exceeding the dissociation 
pressure at fixed temperatures, below the quadruple point. In addition, basic knowledge and experience on the gas hydrates, formed at GZG, Göttingen (Chazallon, 1999; Klapproth, 2003) was available prior to the present work and helped its start.

This thesis work will present the studies which attempt to describe quantitatively the formation process of $\mathrm{CH}_{4}$-gas hydrates as followed by in situ diffraction- and gas consumption experiments starting from a well-characterized ice powder of known structure, grain size, and specific surface area. Additionally to the kinetic studies, ex-situ FE-SEM observations on the gas hydrate formation will be presented (Ch. 3.4). The deduced results will lead to a construction of phenomenological multistage model of gas-hydrate growth from ice powders (Ch. 4). In particular, the model takes into account the observed hydrate-phase expansion beyond the initial ice-grain boundaries into the voids of the initial ice-grain packing and the corresponding reduction of the specific surface of the hydrate shells exposed to the ambient gas. The state-ofthe-art in the understanding of the process of $\mathrm{CH}_{4}$-gas hydrate growth from ice will be given according to the experimental results and their interpretation by the multistage model (Ch. 5). 


\section{References}

Aya, K.; Yamane, K.; Yamada, N. (1992). Stability of clathrate hydrate of carbon dioxide in highly pressurized water. In Fundamentals of Phase Change: Freezing, Melting, and Sublimation - 1992 HTD 215 (P.E. Kroeger and Y. Bayazitogu, eds.), The American Society of Mechanical Engineers, New York, 17-22.

Barrer, R.M.; Edge, A.V.J. (1967). Gas hydrates containing argon, krypton and xenon: kinetics and energetics of formation and equilibria. Proceedings of the Royal Society London A 300, 1-24.

Chazallon, B. (1999). Clathrates d'hydrate d'azote, d'oxigène et d'air - Etude par diffraction neutronique et spectroscopie Raman, Dissertation, Universität Göttingen.

Henning, R.W.; Schultz, A.J.; Thien, Vu; Halpern, Y. (2000). Neutron diffraction studies of $\mathrm{CO}_{2}$ clathrate hydrate: formation from deuterated ice. Journal of Physical Chemistry A, 104, 5066-5071.

Henning, R.W.; Wang, X; Schultz, A.J. (2001). Gas clathrate hydrate research using the high intensity powder diffractometer. IPNS Progress Report, Scientific Instruments \& Highlights, 24-27.

Hwang, M.J.; Wright, D.A.; Kapur, A.; Holder, G.D. (1990). An experimental study of crystallization and crystal growth of methane hydrates from melting ice. Journal of Inclusion Phenomena, 8, 103-116.

Kuhs, W.F.; Dorwarth, R.; Londono D.; Finney, J.L. (1992). In-situ Study on the Composition and Structure of Ar-clathrate. Physics and Chemistry of Ice, (Eds.N.Maeno and T.Hondoh), Hokkaido University Press, Sapporo, 126-130.

Kuhs W.F.; Klapproth, A.; Gotthardt, F.; Techmer, K.; Heinrichs, T. (2000). The formation of meso- and macroporous gas hydrates. Geophysical Research Letters 27(18), 2929-2932.

Klapproth, A.; Goreshnik, E.; Staykova, D.; Klein H.; Kuhs W.F. (2003) Structural Studies of Gas Hydrates. Canadian Journal of Physics, 81, 503-518. 
Klapproth, A. (2002). Strukturuntersuchungen an Methan- und Kohlenstoffdioxid-ClathratHydraten. Dissertation, Universität Göttingen

Mori, Y.H. (1998). Clathrate hydrate formation at the interface between liquid $\mathrm{CO}_{2}$ and water phases: a review of rival models characterizing "hydrate films". Energy Conversion and Management 39(15), 1537-1257.

Mori, Y.H.; Mochizuki, T. (1997). Mass transport across clathrate hydrate films - a capillary permeation model. Chemical Engineering Science 52(20), 3613-3616.

Mori, Y.H.; Mochizuki, T. (2000). Modelling of simultaneous heat and mass transfer to/from and across a hydrate film. Ann. NY Acad. Sci., 912, 633-641.

Moudrakovski, I. L.; C. I. Ratcliffe; J. A. Ripmeester (2002). Application of Magnetic Resonance Microimaging (MRM) to monitor the formation of gas hydrate. Proc. 4th Int. Conf. Gas Hydrates, 444-448.

Salamatin, A. N.; Kuhs W.F. (2002). Formation of porous gas hydrates. Proceedings of the Fourth International Conference on Gas Hydrates, Yokohama, May 19-23, 2002, pp. 766770.

Sloan E. D., Jr. (1998). Clathrate hydrates of natural gases. Second edition, Marcel Dekker Inc.:New York.

Staykova, D.K.; Hansen, T.; Salamatin, A.N.; Kuhs, W.F. (2002). Kinetic diffraction experiments on the formation of porous gas hydrates. Proc. 4th Int. Conf. Gas Hydrates, 537-542.

Staykova, D.K.; Kuhs, W.F.; Salamatin, A.N.; Hansen, Th. (2003). Formation of porous gas hydrates from ice powders: Diffraction experiments and multistage model. Journal of Physical Chemistry B, 107, 10299-10311.

Stern, L.A.; Hogenboom, D.L.; Durham, W.B.; Kirby, S.H.; Chou, I-M. (1998). Optical-cell evidence for superheated ice under gas-hydrate-forming conditions. Journal of Physical Chemistry B 102, 2627-2632. 
Suess, E.; Bohrmann, G.; Rickert, D.; Kuhs, W.F.; Torres, M.E.; Trehu, A.; Linke, P. (2002). Properties and fabric of near-surface methane hydrates at Hydrate Ridge, Cascadia Margin. Proc. 4th Int. Conf. Gas Hydrates, 740-744.

Sugaya, M.; Mori, Y.H. (1996). Behavior of clathrate hydrate formation at the boundary of liquid water and fluorocarbon in liquid or vapor state. Chemical Engineering Science 51(13), $3505-3517$.

Takeya, S.; Hondoh, T.; Uchida, T. (2000). In-situ observations of $\mathrm{CO}_{2}$ hydrate by X-ray diffraction. Annals of the New York Academy of Sciences, 912, 973-982.

Uchida, T.; Hondoh, T.; Mae, S.; Duval, P.; Lipenkov V.Ya. (1992). In-situ observations of growth process of clathrate air-hydrate under hydrostatic pressure. In Physics and Chemisrty of Ice (N. Maeno \& T. Hondoh, eds.), Hokkaido University Press, Sapporo, Japan. 121-125.

Uchida, T.; Hondoh, T.; Mae, S.; Duval, P.; Lipenkov V.Ya. (1994). Effects of temperature and pressure on transformation rate from air bubbles to air-hydrate crystals in ice sheets. Annals of Glaciology 20, 143-147.

Uchida, T.; Kawabata, J. (1995). Observations of water droplets in liquid carbon dioxide. Proceedings of the MARIENV'95 Conference, 906-910.

Wang, X.; Schultz, A.J.; Halpern, Y. (2002). Kinetics of ice particle conversion to methane hydrate. Proc. 4th Int. Conf. Gas Hydrates, 455-460. 


\section{Chapter 2}

\section{Theoretical Section}

\subsection{Thermodynamic Nomenclature}

The process of gas hydrate growth, which can be regarded as a multiphase transition process, is still not well understood. Two main approaches can be used to describe the gas hydrate formation kinetics - a phenomenological, which deals with the properties of material objects at a macroscopic or mesoscopic level, and the microscopic approach, based on an atomic or a molecular conception of mass. The microscopic state of a thermodynamic system reflects the macroscopic behaviour. The basic thermodynamic variables of phase transition processes and parameters necessary for a later discussion of the gas hydrate growth phenomena are given first.

\subsubsection{System and Phase Descriptions}

A thermodynamic (or macroscopic) nomenclature can be used to describe a gas hydrate-forming system. Thermodynamic rules are applicable to macroscopic systems consisting of huge number of particles. Each system and its relation to the surrounding media are described by macroscopic parameters. A set of independent parameters defines a state of a system and determines so called state functions, which are variables independent from the history of the system.

A system, exchanging energy and matter with the surroundings, is an open system. If the system exchanges only energy, it is defined as a closed system. If the system does not interact with the surroundings (isolated system) it has a tendency to reach a state of equilibrium whose parameters do not change with time. This is the most probable macroscopic state which an 
isolated system reaches with time. The state of the system is determined by continuously moving particles that cause a spontaneous deviation from the state of equilibrium (fluctuations). They are neglected within the macroscopic description and cannot be described by the thermodynamic approach. The fluctuations of the macroscopic state of the system can be described only by the microscopic approach. In this sense the descriptions given by the thermodynamics and statistical physics complement each other.

When the system reaches the state of equilibrium, the values of many quantities, uniquely defined by the state, can be measured. Examples of such state variables are the temperature, measuring the intensity of the thermal motion in the system; pressure, dependent on the movement and space distribution of the particles in the system; volume, determined by the outer bodies and so on.

The thermodynamic systems are divided into two classes: homogeneous and heterogeneous systems. A homogeneous system is characterized by a continuous change of its properties during transition between different places within the system. A heterogeneous system consists of a set of homogeneous ones causing a discontinuous change of the system properties. The homogeneous part or phase is a distinct state of the matter in the system. Identical in a chemical composition and physical state, the phase is separated from other material by a phase boundary where a change in a system property and related parameters occurs. The phase boundary has a thickness of about $\mathrm{nm}$, which is comparable to the radius of the molecular interactions. As a result the molecules of the interface interact with the ones of both contacting phases. This makes the properties of the phase boundary different from the ones of the phase volume.

The equilibrium state of one heterogeneous system is characterized by an equivalence of the temperature $T$, pressure $P$ and chemical potential $\mu$ (see Chapter 2.1.2) of the matter in the different phases. For the sake of simplicity the following descriptions are given for two-phase approach but they are applicable for $n$-phase case as well. In case of two-phase system the equilibrium conditions are written as:

$$
\left\{\begin{array}{l}
T_{1}=T_{2} \\
P_{1}=P_{2} \\
\mu_{1}(P, T)=\mu_{2}(P, T)
\end{array}\right.
$$

where 1 and 2 are the phase indices. Detailed analysis of the phase equilibrium and corresponding equations can be found in the book of Landau and Lifshitz (1958). 
The last equation in (1) can be transformed to the equation:

$$
P=P(T)
$$

giving a functional relation between the pressure and temperature of the phases in equilibrium. In the pressure-temperature co-ordinate system the points at which phase equilibrium is possible lie on a curve called phase equilibrium curve (Figure 1).

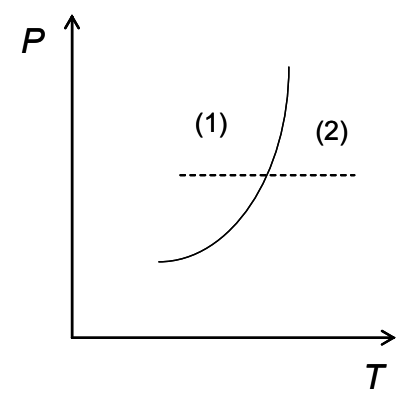

Figure 1 Simplified draw of a phase equilibrium curve in case of thermodynamic system consisting of phases (1) and (2).

When the state of the system considered changes along the dashed line, crossing the curve of equilibrium, a coexistence of both phases in equilibrium occurs at the cross-point and after that the system undergoes a transition to another phase. The phase equilibrium curve can be regarded as a stability limit of a phase with respect to its discontinuous changes.

Diagrams containing information about phases, which are present at various conditions, are called phase diagrams. A driving force for a phase transformation appears if the conditions of a system are changed in such a way that the system moves from one phase field into another in the phase diagram.

\subsubsection{Phase Transitions - Basic Concepts}

Some additional thermodynamic parameters have to be introduced in relation to the basic descriptions of the phase transition processes.

The internal energy $U$, described microscopically as a sum of the energies of different motions and interactions of the molecules of a system, is an important state function. Thermodynamics describes the internal energy through its change $\Delta U$ which is defined as the difference between the heat absorbed by the system $Q$ and the external work done by the system $W$ (first law of thermodynamics). The internal energy is a state function of temperature and pressure which are variables controlled in the laboratory. The equation 


$$
U=U(T, P)
$$

called caloric equation of state together with the equation

$$
V=V(T, P)
$$

called thermal equation of state describe the simplest thermodynamic system which is one-phase system determined by two parameters.

A closed system can undergo an irreversible (reversible) process of transition from one state to another if the system can return to its initial state with (without) a change in the surroundings, respectively. A measure of the irreversibility of such a process is the change of a state function called entropy $S$, related to the heat received by the system:

$$
\Delta S=Q / T
$$

According to the statistical physics the entropy of the system in a certain state characterizes the probability of this state:

$$
S=k_{B} \ln w
$$

where $k_{B}$ is Boltzmann constant and $w$ is determined by the number of microstates realising a macrostate. As a result of a trend from a less probable to a more probable state of a closed system its entropy increases to a maximum in equilibrium state.

Other state functions, describing systems at different conditions, are the thermodynamic potentials (or characteristic state functions) introduced on the basis of the first law of thermodynamics.

A characteristic function of the independent variables $V$ and $T$ is determined as: $F=U-T S$ called Helmholtz energy (or Free energy). In case of simple system, a characteristic function of independent variables $T$ and $P$ is introduced as: $G=U-T S+P V$, called Gibbs energy $G$, whose differential is: $d G=-S d T+V d P$. This function is important because $T$ and $P$ are the variables, which are most easily controlled experimentally, and the equilibrium is reached when $G$ reaches its minimum at a constant temperature and pressure. In case of system, consisting of variable amount of particles, the differential of the Gibbs energy has the following form: $d G=-S d T+V d P+\sum_{i} \mu_{i} d N_{i}$, where $\mu_{i}$ is called chemical potential of $i$-type particles. For a system of one-type particles the chemical potential $\mu=\left(\frac{\partial G}{\partial N}\right)_{P, T}=f(T, P)$ is equal to the 
Gibbs energy of one particle and it depends on the temperature and pressure but not on the number of the particles.

A change of the ambient conditions of a heterogeneous system in equilibrium could cause transitions of the matter from one phase to another, i.e. phase transitions. For a body in equilibrium as well as in a state of phase transition, the temperature, pressure and chemical potential of the matter in the different phases are constant. The behaviour of the derivatives of the Gibbs energy determines the type of an occurring phase transition. Discontinuous (or first-order) phase transitions are characterized by jumps in the first-order derivatives of the Gibbs energy: $V=\left(\frac{\partial G}{\partial P}\right)_{T}, S=-\left(\frac{\partial G}{\partial T}\right)_{P}$. These transitions are accompanied by a discontinuous change of the relative volume or the density of matter and consumption or release of heat. Continuous (or second-order) phase transitions keep continuous the first-order derivatives but cause jumps in the second order derivatives of the Gibbs energy: heat capacity at constant pressure $C_{P}=-T\left(\frac{\partial^{2} G}{\partial T^{2}}\right)_{P}$, isothermal compressibility $\beta_{T}=-\frac{1}{V}\left(\frac{\partial^{2} G}{\partial P^{2}}\right)_{T}$, and thermal expansivity $\alpha=\frac{1}{V}\left(\frac{\partial^{2} G}{\partial T \partial P}\right)$. When these derivatives become infinite the transitions are called critical.

The first-order phase transitions are described by a differential equation known as Clausius-Clapeyron equation. It is obtained as a result of the differentiation of the equilibrium condition for the chemical potentials (the last equation of (1)) and it is regarded as a differential equation of the phase equilibrium curve:

$$
\frac{d P}{d T}=\frac{q}{T\left(v_{2}-v_{1}\right)}
$$

where $q=T\left(s_{2}-s_{1}\right)$ is the latent heat of the transition from one phase to another; and $v_{2}-v_{1}$ is the resulting volume change per unit mass of a substance. The Clausius-Clapeyron equation written in the form:

$$
\frac{d T}{d P}=\frac{T\left(v_{2}-v_{1}\right)}{q}
$$

determines the variation of the phase-transition temperature with the pressure.

Equations for the second order and critical phase transitions are described in Landau \& Lifshitz (1958) and Bazarov (1986). 


\subsubsection{Driving Force}

The irreversible processes, defined in the previous section, are studied by thermodynamics of irreversible processes - an extension of the classical thermodynamics, using the following assumptions. The first one supposes the entropy produced by an irreversible process determines the driving force of this process. The second one defines the driving force of an irreversible process as a thermodynamic property of a state of non-equilibrium. The third one is the assumption that the rate of a process is proportional to the driving force, at least for low values of the driving force.

When an irreversible process occurs inside the system, internal entropy is produced

$$
(d S)_{i p}>0
$$

where ip indicates the internal process. If there is a simultaneous heat exchange with the surroundings, the total change in the system during the irreversible process is described by:

$$
d S=d Q / T+(d S)_{i p}>d Q / T
$$

which in case of reversible process $(d S)_{i p}=0$ transforms to $d S=d Q / T$.

An internal process will continue as long as $(d S)_{i p}>0$ (second law of thermodynamics). It will stop when the internal entropy becomes $(d S)_{i p} \leq 0$. The driving force $f$ for any internal process is:

$$
f=T \cdot \frac{(d S)_{i p}}{d \chi}
$$

where $\chi$ is a variable describing the extent of a certain internal process; and $(d S)_{i p} / d \chi$ is the derivative of the internal entropy produced by this process. For a system, which is not in equilibrium, $(d S)_{i p}>0$ and thus $f d \chi>0$. In case of $i$ number of internal processes $f d \chi$ has to be replaced by $\sum f_{i} d \chi_{i}$. If the rate of reaction is denoted by $d \chi_{i} / d t$ it is proportional to the driving force $\frac{d \chi_{i}}{d t}=k_{i} \cdot f_{i}$ with a coefficient $k_{i}$. The entropy of a process is produced with a rate deduced from $T \cdot \frac{(d S)_{i p}}{d t}=f_{i} \cdot \frac{d \chi_{i}}{d t}=k_{i} \cdot f_{i}^{2}$. A process will proceed when $d \chi$ and $f$ have the same sign. By convention $d \chi$ has a positive value in the studied direction and $f>0$ for a spontaneous process in that direction. The process proceeds until it approaches a state of equilibrium where $f=0$. The 
driving force refers to the difference between the system's state and the equilibrium one. The higher the difference the higher rate of transition to the equilibrium is.

The driving force is given by the change of characteristic state function of independent state variables, which are constants during the reaction. Reaction occurring in every point of a system with a constant rate everywhere is classified as a homogeneous reaction. Reaction, occurring at the phase boundaries of a system, is called heterogeneous reaction or phase transformation. It results in growth of some phase and shrinking of another. In most cases, a heterogeneous reaction is accompanied by a change in the system composition and occurs under diffusion. In general, it occurs at the places of composition difference. As result of the diffusion the local differences decrease and eventually disappear.

\subsubsection{Activation Energy and Nucleation}

When the particles of a reacting system (reactant) undergo a transformation to a product system (product) they follow a path of least resistance. This path is energetically favourable but may still require a lot of energy. The energy required by a system to undergo a particular process is called activation energy and it is measured as an excess energy over the one of the ground state of the system. In allusion to the mechanical kinetic and potential energy the energy of a system consists of free energy and energy of bonds between the particles. In this sense the activation energy is minimum amount of free energy necessary to break the bonds between the reactant particles and to form new bonds of the product. The following figure shows a drawing of the reaction path in case of exothermic reaction (reactant energy > product energy). It is accompanied by a release of heat equal to the energy difference between the reactant and the product.

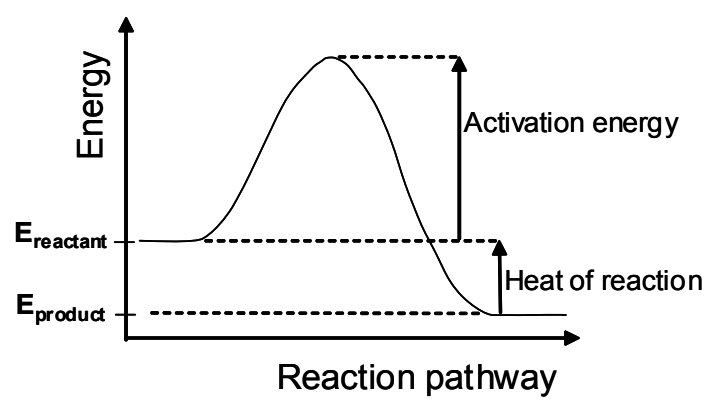

Figure 2. Pathway of an exothermic reaction. The activation energy is determined by the energy difference between the reactants and the activated species. 
The reaction path controls the rate of one reaction. One path with high activation energy would lead to a relatively slow reaction. A relatively fast reaction would proceed along a path with small activation energy. One reaction with a pathway, consisting of few steps with different activation energies (Figure 3 ), is called a multi-step reaction.

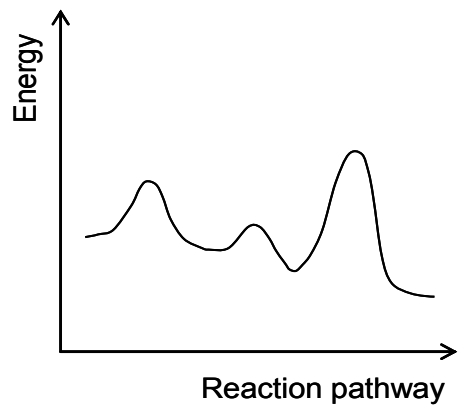

Figure 3. Possible pathway of a multi-step exothermic reaction. The third step with the highest activation energy is the rate-determining step.

One sequence of steps through which the reaction proceeds is called reaction mechanism. The reaction step with the smallest rate caused by the highest activation energy controls the overall rate of the process. This step is called rate-determining step. For example when a phase transformation proceeds together with diffusion and the process occurring at a phase interface is rapid in comparison with the diffusion then the rate-determining step is the diffusion. In this case the transformation process is diffusion-controlled.

The reaction rate is also influenced by the temperature. The temperature effect on the reaction rate originates from the temperature effect on the equilibrium constant:

$$
\frac{d(\ln K)}{d(1 / T)}=-\frac{H}{R}
$$

where $R$ is the gas constant and $H$ is the heat of reaction. The equilibrium constant $K$ is defined as $K=k_{1} / k_{2}$, where $k_{1}$ and $k_{2}$ is the rate constant for the forward and the reverse reaction, respectively. The total reaction can be schematically written as $A+B \underset{k_{2}}{\stackrel{k_{1}}{\Leftrightarrow}} A B$, where $A$ and $B$ are the reactants and $A B$ is the product. Substituting $K$, the equation (12) transforms to:

$$
\frac{d\left(\ln k_{1}\right)}{d(1 / T)}-\frac{d\left(\ln k_{2}\right)}{d(1 / T)}=-\frac{H}{R}
$$

Arrhenius (1889) divided Eq. (13) into two parts, each having a form: 


$$
\frac{d(\ln k)}{d(1 / T)}=-\frac{E_{a}}{R}
$$

where $E_{a}$ is the activation energy. Taking $E_{a}$ as a constant, Eq. (14) can be integrated. As a result:

$$
\ln k=\ln A-E_{a} / R T
$$

where $\ln A$ is a constant of integration. Finally this relation can be transformed to:

$$
k=A \exp \left(-E_{a} / R T\right)
$$

known as Arrhenius equation. The term $A$ is a pre-exponential factor. The rate constant of the reaction at each temperature is determined by Eq. (16) resulting from the linear relation between the logarithm of the rate constant and the reciprocal value of the temperature (Eq. (15)). The values of $A$ and $E_{a}$ can be calculated from the linear fit of minimum three rate constants determined at three different temperatures. Figure 4 shows an example plot that can give the rate constant $k_{0}$ at any temperature of interest $\mathrm{T}_{0}$ by the Arrhenius equation.

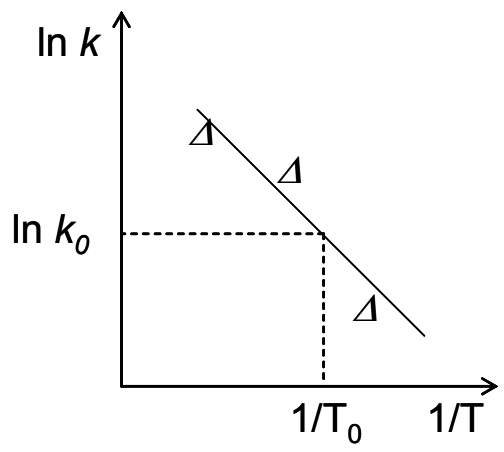

Figure 4. Logarithmic plot of example rate constants $k$ obtained experimentally (triangles) can be used to calculate the parameters in the Arrhenius equation (linear fit).

One phase transition proceeds through nucleation and growth. The first energetic barrier, which a system has to pass in order to undergo a phase transition process, is the nucleation barrier. It is related to the interface between the different phases in the system. A one-phase system, having free energy larger than the one of a multi-phase system of the same particles, can exist in a fixed volume for a certain time period. Such a state is called metastable and the system tends to leave it in order to reach a stable multi-phase state of a minimum free energy. The change from the metastable to the stable state occurs as a result of fluctuations in the homogeneous medium forming small quantities of the new phase, or nuclei. As a result the Gibbs energy of the system changes in two directions: it decreases, proportionally to the nuclei volume; and increases, proportionally to the nuclei surface. One process of creation of an interface, 
causing an increase of the free energy of the system, is energetically unfavourable. As a result the nuclei, which are very small, are unstable and disappear and the origin phase is stable with respect to the internal fluctuations. For each metastable state a minimum size of a nucleus, which is stable within the initial phase, exists. It is called critical size $a_{c r}$ and the nuclei of this size are called critical nuclei. The critical size of the nucleus is related to the change of the Gibbs energy as it shown schematically in Figure 5.

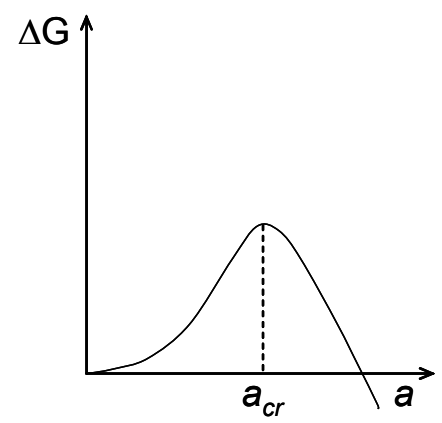

Figure 5. Nuclei of size below the critical one are energetically unfavourable and disappear. Only the bigger nuclei can continue to grow.

A nucleus of size $a<a_{c r}$ is unstable because its appearance causes an increase of the Gibbs energy of the system. Only a nucleus of size $a>a_{c r}$, decreasing the Gibbs energy, is stable and continues to grow spontaneously. The critical value of the nucleus size can be obtained from the condition:

$$
\left.\frac{\partial(\Delta G)}{\partial a}\right|_{a=a_{c r}}=0
$$

This interfacial energy effect, which the system has to overcome in the phase transition process, can be regarded as a nucleation barrier. Once the system passed this barrier the thermodynamically favourable new phase will start to grow.

\subsection{Gas Phase}

The behaviour of an ideal gas is a good approximation of the one of a real gas at low pressures. An ideal gas phase consists of atoms or molecules of negligible sizes, which do not exert intermolecular forces and interact through elastic collisions. The internal energy of an ideal gas is its kinetic energy and any change in the internal energy leads to a change in the temperature. The 
state of the ideal gas is characterised by the state variables - absolute pressure $P$, volume $V$ and absolute temperature $T$, related in Ideal Gas Law:

$$
P V=n R T=N k_{B} T
$$

where $n$ and $\mathrm{N}$ are numbers of moles and molecules, respectively. The pressure $P$ of an ideal gas at a given temperature $T$ is proportional to the concentration of the gas molecules or the gas density $N / V$.

The ideal gas is a theoretical abstraction, which would obey Eq. (18) under all conditions. Only at low pressures the behaviour of a real gas is close to the one of an ideal gas. Thus, a variable fugacity is a substitute for pressure in the thermodynamic equations of the real gases. The fugacity has units of pressure and it is a function of pressure. As it is given by Sloan (1998) the fugacity of component $i$ in a phase $z\left(f_{i}^{z}\right)$ is related to the pressure $P$ through the equation $f_{i}^{z}=w_{i} \phi_{i}^{z} P$, where $w_{i}$ is the mole fraction of the component $i$ and $\phi_{i}^{z}$ is the fugacity coefficient. For pure gases, like for example methane and carbon dioxide, the fugacity can be deduced from tabulated values of fugacity/pressure ratio $(f / P)$ calculated at different pressures and temperatures using the corresponding equations of state (Intern. Thermodyn. Tables, 1973, 1976).

\subsection{Ice Ih Phase}

The solid state of the water, ice, consists of water molecules arranged in a way that each water molecule is connected to four others by hydrogen bonds in a tetrahedral framework. An elementary arrangement is shown in Figure 6, giving a spatial distribution of five water molecules in the ice.

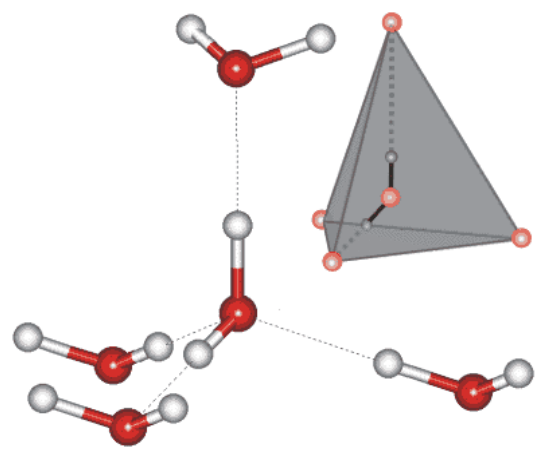

Figure 6. Water molecules of the ice phase make a tetrahedral arrangement. The image source is: http://www.lsbu.ac.uk/water/hbond.html 
Each water molecule has an oxygen atom (larger ball, Fig.6) connected to two hydrogen atoms (smaller balls, Fig.6) by covalent bonds. The hydrogen bond (marked as a dotted line on the Fig. 6), connecting two water molecules, has an essential electrostatic character. It binds the hydrogen nucleus, which belongs to one molecule (hydrogen donor) to lone-pair electrons of oxygen from another molecule (hydrogen acceptor). Each oxygen atom, having six outer electrons, uses two of them to make covalent bonds and four (as two lone-pairs) to make two hydrogen bonds. Each hydrogen atom participates in one covalent and one hydrogen bond. The tetrahedral arrangement of the water molecules in this state is a result of the interactions of the oxygen and hydrogen atoms with the atoms of the neighbouring molecules.

\subsubsection{Bulk Structure and Surface}

One stable at an ambient pressure crystalline form of ice, in which the oxygen atoms of the water molecules are arranged in layers of hexagonal rings, is known as a hexagonal ice Ih with a space group $\mathrm{P}_{3} / \mathrm{mmc}$ (see Fig. 7). The atoms of each hexagonal ring are displaced with respect to each other alternately in two planes. The resulting hexagonal channels make ice Ih an open structure.
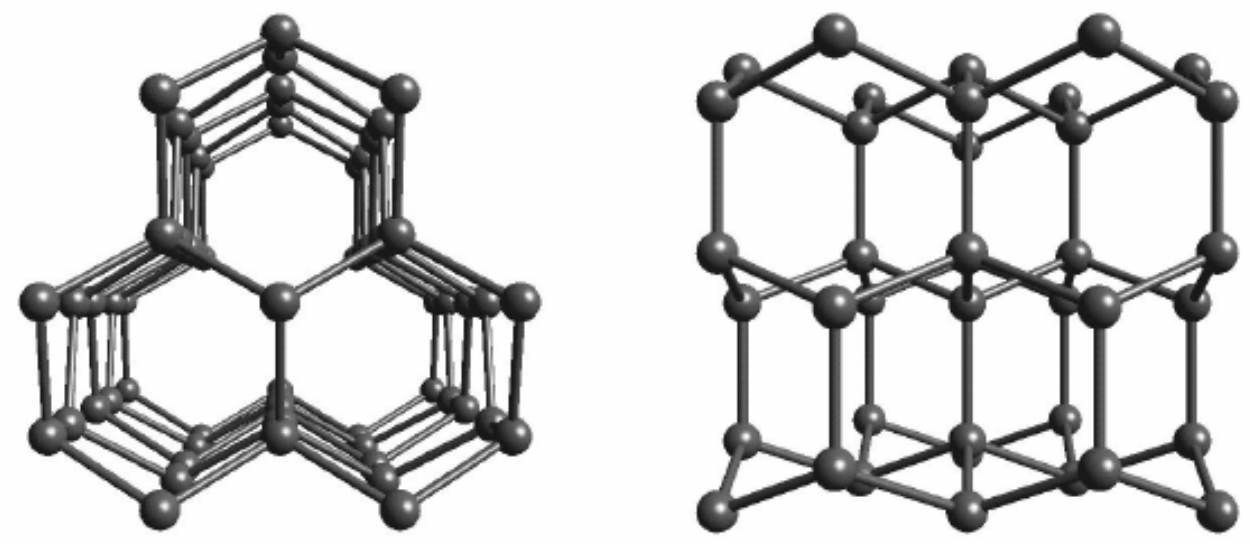

Figure 7. Structure of ice Ih, given by Lobban (1998). Right and left draw show a structure view parallel and perpendicular to the hexagonal channels, respectively.

In reality the water molecules undergo small displacements from the shown positions. The arrangement on Fig. 7 should be regarded as an average space- and time- structure. More details 
about the hexagonal ice structure and its properties are described by Kuhs and Lehmann (1986); Petrenko and Whitworth (1999).

The surface of ice is an essential parameter in the kinetics of the ice-phase transition processes. The structure and the mobility of the water molecules of the ice surface may play an important role also in the kinetics of the clathrate formation at temperatures below $0^{\circ} \mathrm{C}$. The water molecules in the ice surface are poorly bounded because they experience bonding forces to the other molecules only from one side. It makes the structure of the free surface to some extent different from the one of the bulk. A number of experimental and theoretical studies on the structure and the physical properties of the ice surface, reviewed by Petrenko and Whitworth (1999), showed the importance and complexity of such investigations, especially close to the melting point of ice. Dash (1995) and Wettlaufer (1997) discussed theoretically the phenomenon of surface premelting or the existence of a liquid-like surface film at temperatures and pressures below the phase boundaries, corresponding to the bulk transitions. The importance of the thermodynamical properties of a water layer at the ice surface for the kinetic processes of growth of snow crystals and frost heaving is discussed by Kuroda (1987). Bluhm et al. (2002) presented experimental observations on the premelting of ice showing an existence of a liquid-like film at a temperature interval of $-20^{\circ} \mathrm{C}$ to $0^{\circ} \mathrm{C}$. When the temperature approached the melting point of ice the film was about $20 \AA$ thick and at $253 \mathrm{~K}$ - almost $0 \AA$. Likewise, considering the papers of Mizuno and Hanafusa (1987), Wei et al. (2000) and Sloan and Fleyfel (1991), it can be concluded that the existence, structure and the temperature dependence of the quasi-liquid layer of water molecules at the ice interfaces may play a key role in the rearrangement of the water molecules during hydrate growth. Thus, the ice surface is considered as an essential factor in the formation of gas hydrates below $0^{\circ} \mathrm{C}$.

\subsubsection{Phase Diagram}

The water molecules in a solid state form different crystallographic structures depending on the thermodynamic conditions. The P-T conditions of stability of the different crystalline phases of ice are shown on the phase diagram (Figure 8). 


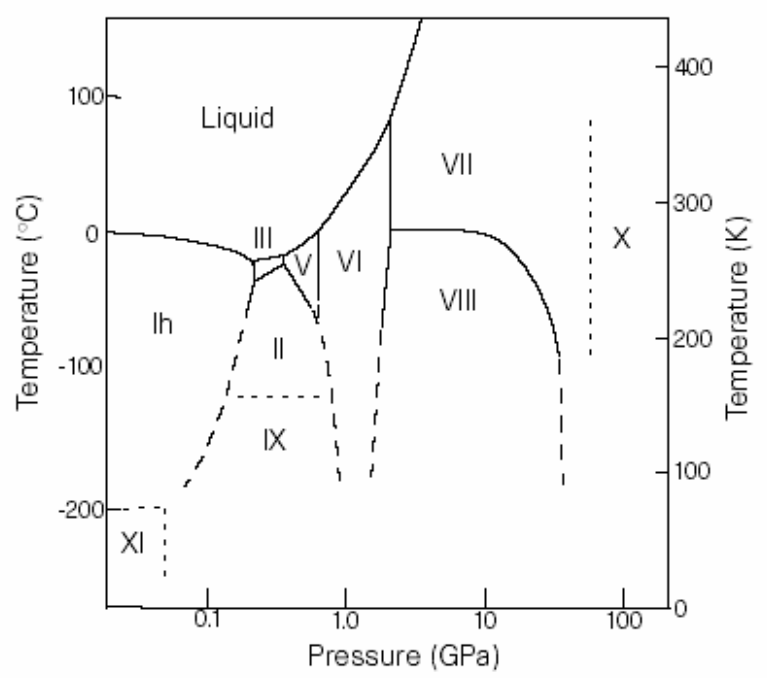

Figure 8. Ice phase diagram taken from Lobban et al., 1998. Solid lines represent measured transitions; dashed lines, transitions extrapolated to low temperatures; dotted lines, predicted transitions.

Each line gives the conditions of coexistence of two ice phases. The point where three lines join, called triple point, denotes the coexistence of three phases of ice. The ice phase, used to form gas hydrates during kinetic experiments, was the hexagonal ice Ih that is stable at temperatures below $273 \mathrm{~K}$ and an ambient pressure.

\section{4 $\mathrm{CH}_{4}^{-}$and $\mathrm{CO}_{2}$ - Gas Hydrate Phases}

Gas hydrates or clathrate hydrates are ice-like crystalline solids consisting of hydrogen bonded water molecules arranged in a host lattice encaging small, usually apolar guest molecules. These solid phases exist as stable compounds at high gas pressures and/or low temperatures. The following sections introduce the phases whose growth kinetics was observed experimentally during $\mathrm{CH}_{4-}$ and $\mathrm{CO}_{2-}$ gas hydrate formation.

\subsubsection{Structure}

The majority of gas hydrates are observed to form two main crystallographic structures - von Stackelberg cubic structures I and II of space group $\mathrm{Pm} \overline{3} \mathrm{n}$ and $\mathrm{Fd} \overline{3} \mathrm{~m}$, respectively (Stackelberg and Müller, 1954) (see Fig. 9). 


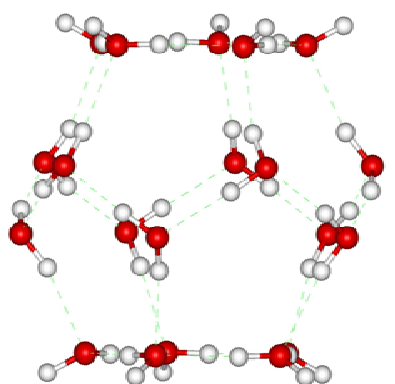

$5^{12}$

(Small cage)
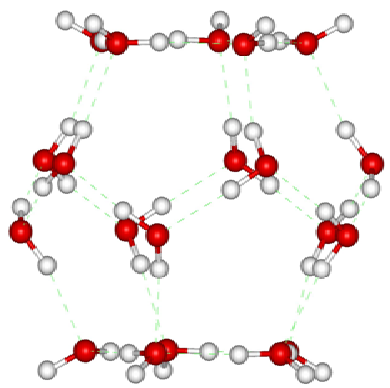

$5^{12}$ (Small cage)

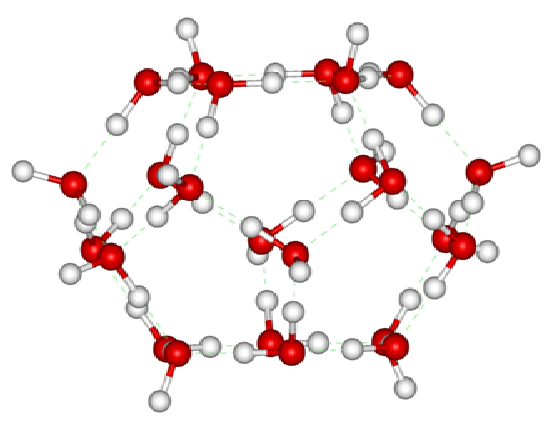

$5^{12} 6^{2}$

Structure I

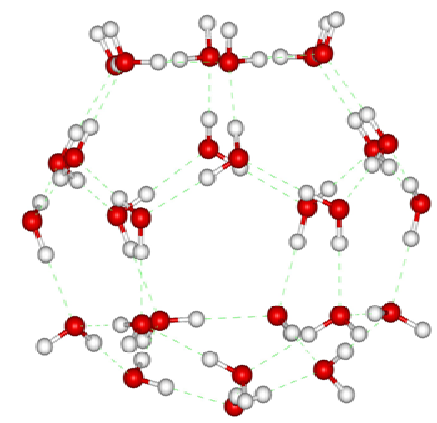

51264

Structure II $\quad$ (Large cage)

Figure 9. Water molecules build small and large cavities in the gas hydrate structure I and II. Oxygen atoms at the vertices have three hydrogen bonds along the edges of the cage (dashed lines). The remaining hydrogen bond is a part of another cage. The picture is provided by Dr. V. Chihaia, GZG-Abt. Kristallographie.

The unit cell of structure I (type I) has 46 water molecules forming two types of cavities as follows - 2 small cages of pentagonal dodecahedron $\left(5^{12}\right)$ and 6 large cages of tetrakaidecahedron $\left(5^{12} 6^{2}\right)$. Each $5^{12}$ unit is built by twelve pentagons. The $5^{12} 6^{2}$ cavity is formed by two opposite hexagonal faces and twelve pentagonal faces. The unit cell of structure II (type II) is made by 136 water molecules arranged in a framework with two types of cavities as well 16 small cages of pentagonal dodecahedron and 8 larger ones of hexakaidecahedron $\left(5^{12} 6^{4}\right)$. The $5^{12} 6^{4}$ cavity has four hexagonal faces and twelve pentagonal faces.

The cavities of the structures I and II can encage different guest molecules. For example the methane (see Fig. 10) and carbon dioxide molecules can be incorporated in the cavities of gas hydrate structure I. 

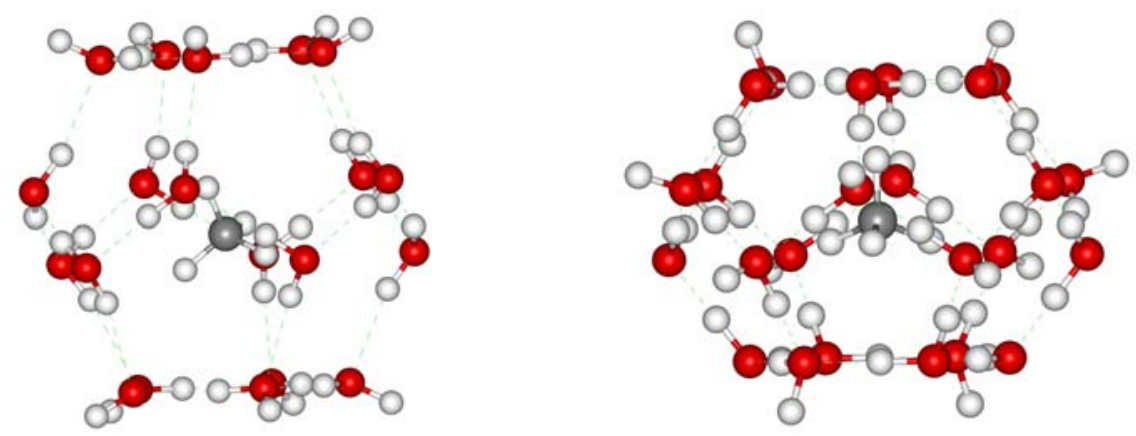

Figure 10. Methane molecules trapped in the cavities of structure I. The carbon atom (a big grey ball) connected to four hydrogen atoms (small white balls) show the methane molecule inside the small (left image) and large (right image) cages. The picture is provided by Dr. V. Chihaia, GZG-Abt. Kristallographie.

Structure II is favourable for hydrate formers as the nitrogen and oxygen and for larger hydrocarbons. Recent intensive studies of the structures I and II and their physico-chemical properties are presented in the PhD Thesis works of Klapproth (2002) and Chazallon (1999), respectively.

\subsubsection{Phase Stability}

The structures described above are stable at different temperature and pressure conditions depending on the gas hydrate former. The thermodynamic conditions of stability of the gas hydrate structures formed by methane and carbon dioxide are shown on Figure 11 and 12, respectively.

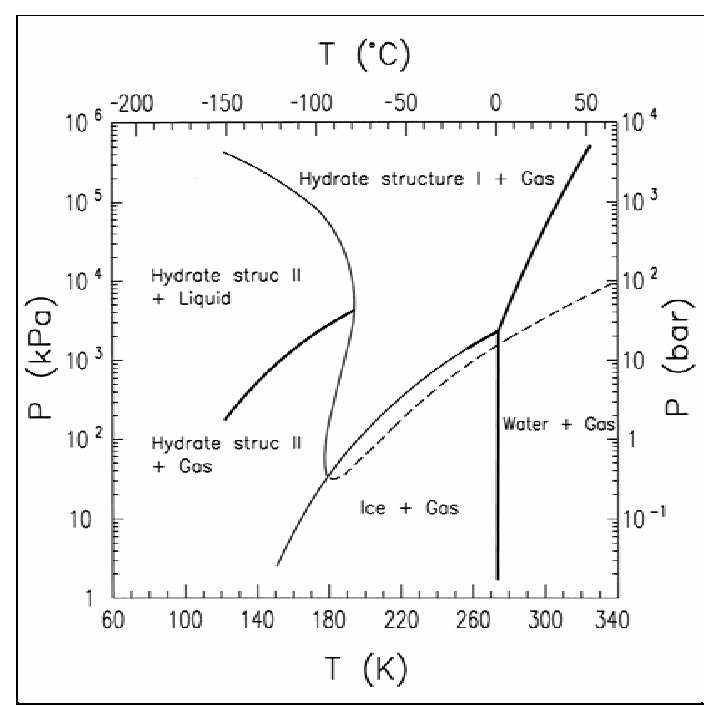

Figure 11. Phase diagram of the methane gas hydrate. Bold lines represent well-established phase boundaries. Thin lines are calculated boundaries as obtained from the statistical thermodynamic theory of van der Waals and Platteeuw (1959) and the separation of the methane hydrate typeI/typeII as calculated by Lundgard and Mollerup (1992). 
The quadruple point $\left(\mathrm{I}-\mathrm{L}_{\mathrm{w}}-\mathrm{H}-\mathrm{V}\right)^{*}$ of methane hydrate is $(\mathrm{T}=272.9 \mathrm{~K} ; \mathrm{P}=2.563 \mathrm{MPa})$ (Sloan, 1998).

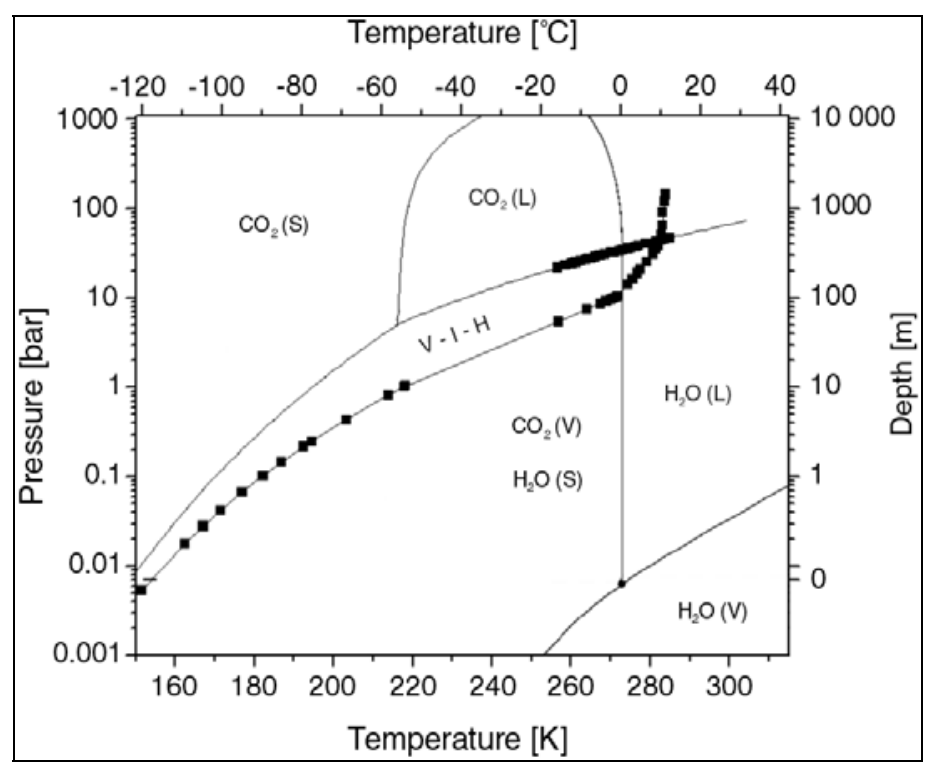

Figure 12. $\mathrm{CO}_{2}$ gas hydrate phase diagram. Black squares show measured data (after Sloan, 1998), which mark the lower stability limit of the $\mathrm{CO}_{2}$ gas hydrate. The lines drawing $\mathrm{CO}_{2}$ phase boundaries are calculated according to the Intern. Thermodyn. Tables (1973). The water phase boundaries are only schematically shown. Picture is provided by G.Genov, GZG-Abt. Kristallographie.

The $\mathrm{CO}_{2}$-hydrate has two quadruple points as follows: $\left(\mathrm{I}-\mathrm{L}_{\mathrm{w}}-\mathrm{H}-\mathrm{V}\right)(\mathrm{T}=273.1 \mathrm{~K} ; \mathrm{P}=1.256 \mathrm{MPa})$ and $\left(\mathrm{L}_{\mathrm{w}}-\mathrm{H}-\mathrm{V}-\mathrm{L}_{\mathrm{HC}}{ }^{*}\right)(\mathrm{T}=283.0 \mathrm{~K} ; \mathrm{P}=4.499 \mathrm{MPa})$ (Sloan, 1998). Thus, an interval of pressure providing the hydrate stability is much more narrow for $\mathrm{CO}_{2}$ - than for $\mathrm{CH}_{4}$-hydrates.

\subsection{Gas Hydrate Formation - Microscopic Conception}

One process of crystallization involves two steps: nucleation and growth. A unified molecular picture of the formation of gas hydrate structure is not available yet and it is still object of a research.

Labile cavity-like clusters of water molecules around apolar molecules, formed in a liquid film at the ice-gas interface, are considered as basic units for a subsequent nucleation of the hydrate structures. Using ab initio calculations Chihaia et al., (2004) obtained that water clusters

\footnotetext{
${ }^{*} \mathrm{I}$-ice, $\mathrm{L}_{\mathrm{w}}$-water, H-hydrate, $\mathrm{V}$-vapor

${ }^{*} \mathrm{~L}_{\mathrm{HC}}$-hydrocarbon liquid phase
} 
$\left(\mathrm{H}_{2} \mathrm{O}\right)_{n}, \mathrm{n}=20$ and $\mathrm{n}=24$, in dodecahedral and tetrakaidecahedral arrangement appear as stable isolated structures. If they were in a water phase they would start to interact with the neighbour molecules, which would destroy their shape. Some of the clusters have a higher or a lower stability depending on the arrangement of their protons. Likewise, Belosludov et al. (2002) studied different cage-like water clusters and showed that the first step of formation of methane hydrate is the formation of small $\left(5^{12}\right)$ water cavities filled with guest molecules. Moreover, it was evidenced by a NMR work of Pietrass et al. (1995) and Moudrakovski et al. (2001) that the small cages seem to be preferred for the hydrate nucleation. In his labile cluster hypothesis Sloan (1998) considered a possible nucleation pathway: labile clusters, made by one guest molecule encaged by 20 to 24 water molecules, diffuse in a liquid phase and agglomerate causing formation of a critical nucleus. However, the labile cluster hypothesis is nowadays considered as an unlikely scenario for hydrate formation. Radhakrishnan and Trout (2002) studied the free energy surface for nucleation of $\mathrm{CO}_{2}$ clathrate at the liquid $\mathrm{CO}_{2}$-liquid $\mathrm{H}_{2} \mathrm{O}$ interface and presented a mechanism of hydrate nucleation according to local structuring hypothesis. It assumed a local ordering of water molecules around $\mathrm{CO}_{2}$ molecules, leading to the formation of a critical clathrate nucleus. Both hypotheses described the water clusters but in different possible arrangements. The second one considers water clusters, arranged in a stable configuration, which are rigid (or structured). The first one regards such arrangements of water clusters, which are not so stable (labile). One hypothetical picture of gas hydrate crystal growth from solution by means of an incorporation of clusters into the growing crystal surface is discussed by Sloan (1998). The nucleation and the induction period of the gas hydrate formation in aqueous solutions are theoretically formulated in the papers of Kashchiev and Firoozabadi (2002, 2003).

More experimental and theoretical work is necessary to provide one detailed molecular picture of the self-controlled gas hydrate nucleation and growth. It would clarify the macroscopic mechanisms determining the clathrate formation kinetics in different thermodynamic systems.

\subsection{Conclusion}

The phase transition process of gas hydrate growth from ice requires water molecules to change their arrangement from one of the hexagonal ice to that of the cubic clathrate structure cages surrounding guest molecules. It needs the hydrogen bonds between the water molecules in the ice 
structure to be destroyed and the new ones in the clathrate structure to be made, i.e. activation energy is required. How it is happening on a molecular level is still an open question. A macroscopic investigation of the kinetics of $\mathrm{CH}_{4}$ - and $\mathrm{CO}_{2}$ - clathrate growth from ice Ih is the object of the following experimental studies. The temperature dependence of the studied process can be used to obtain activation energy of each rate-determining step. This knowledge could help to clarify what mechanism controls the kinetics of gas hydrate formation within a particular thermodynamic system. 


\section{References}

Arrhenius, S. A. (1889). Über die Reaktionsgeschwindigkeit bei der Inversion von Rohrzucker durch Säuren. Z. Phys. Chem., 4, 226-248.

Bazarov, I.P.; Gevorkian, E.V.; Nikolaev, P.N. (1986). Thermodinamika i Statisticheskaya Fizika. Teoria ravnovesnah sistem. Izdatelstvo Moskovskovo Gosudarstvenovo Universiteta (in Russian).

Belosludov R.V.; Kawazoe, Y.; Belosludov V.R.; Kuznetsov, F. A. (2002). Role of guest molecules on the stability of different cagelike structures of large water clusters investigated by first-principles calculations. Proc. 4th Int. Conf. Gas Hydrates, 683-686.

Bluhm, H.; Ogletree, D. F.; Fadley, Ch. S.; Hussain, Z.; Salmeron, M. (2002). The premelting of ice studied with photoelectron spectroscopy. J. Phys.: Condens. Matter, 14, L227-L233.

Chazallon, B. (1999). Clathrates d'hydrate d'azote, d'oxigène et d'air - Etude par diffraction neutronique et spectroscopie Raman. Dissertation, Universität Göttingen.

Chihaia, V.; Adams, S.; Kuhs, W. F. (2004). Influence of water molecules arrangement on structure and stability of $5^{12}$ and $5^{12} 6^{2}$ buckyball water clusters. A theoretical study. Chemical Physics, 297, 271-287.

Dash, J. G.; Fu, Haiying ; Wettlaufer, J. S. (1995). The premelting of ice and its environmental consequences. Rep. Prog. Phys., 58, 115-167.

International thermodynamic tables of the fluid state. [Vol.] 3: Carbon Dioxide (1973). International Union of Pure and Applied Chemistry, Pergamon Press.

International thermodynamic tables of the fluid state. [Vol.] 5: Methane (1976). International Union of Pure and Applied Chemistry, Pergamon Press.

Kashchiev D.; Firoozabadi, A. (2002). Driving force for crystallization of gas hydrates. Journal of Crystal Growth, 241, 220-230.

Kashchiev D.; Firoozabadi, A. (2002). Nucleation of gas hydrates. Journal of Crystal Growth, 243, 476-489. 
Kashchiev D.; Firoozabadi, A. (2003). Induction time in crystallization of gas hydrates. Journal of Crystal Growth, 250, 499-515.

Klapproth, A. (2002). Strukturuntersuchungen an Methan- und Kohlenstoffdioxid ClathratHydraten. Dissertation, Universität Göttingen.

Kuhs, W. F.; Lehmann, M. S. (1986). The structure of ice-Ih. In Water Science Reviews 2, F. Franks, ed., Cambridge University Press, 1-65.

Kuroda, T. Role of water layer at an ice surface in the kinetic processes of growth of ice crystals - growth of snow crystals and frost heaving. Journal de Physique, Colloque C1, Supplement au N3, 48, C1-487-C1-493.

Landau, L.D.; Lifshitz, E.M. (1958). Statistical Physics. Vol. 5 of Course of theoretical physics. Pergamon press Ltd.

Lobban C. (1998) Neutron diffraction studies of ices. Ph.D. Thesis, University College London.

Lobban, C; Finney, J. L.; Kuhs, W.F. (1998). The structure of a new phase of ice. Nature 391, $268-270$.

Lundgaard, L.; Modellrup, J. (1992). Calculation of phase diagrams of gas-hydrates. Fluid Phase Equilibria, 76, 141-149.

Mizuno, Y.; Hanafusa, N. (1987). Studies of surface properties of ice using nuclear magnetic resonance. Journal de Physique, Colloque C1, Supplement au N3, 48, C1-511-C1-517.

Moudrakovski, I.L.; Sanchez, A.A.; Ratcliffe, C.I.; Ripmeester, J.A. (2001). Nucleation and growth of hydrates on ice surfaces: new insights from ${ }^{129} \mathrm{Xe}$ NMR experiments with hyperpolarized xenon. J. Phys. Chem. B 105, 12338-12347.

Petrenko, V. F.; Whitworth, R. V. (1999). Physics of ice. Oxford University Press.

Pietrass, T.; Gaede, H.C.; Bifone, A.; Pines A.; Ripmeester, J.A. (1995). Monitoring xenon clathrate hydrate formation on ice surfaces with optically enhanced ${ }^{129}$ Xe NMR. Journal of American Chemical Society 117, 7520-7525. 
Radhakrishnan R.; Trout, B. L. (2002). A new approach for studying nucleation phenomena using molecular simulation: Application to $\mathrm{CO}_{2}$ hydrate clathrates. Journal of Chemical Physics, 117, No4, 1786-1796.

Sloan E. D., Jr. (1998). Clathrate hydrates of natural gases. Second edition, Marcel Dekker Inc.:New York.

Sloan, E.D., Jr.; Fleyfel, F. (1991). A molecular mechanism for gas hydrate nucleation from ice. American Institute of Chemical Engineering Journal 37, 1281-1292.

van der Waals, J. H. and Platteeuw, J. C. (1959) Clathrate solutions. Adv. Chem. Phys. 2, 1-57.

von Stackelberg, M. and Müller, H. R. (1954) Feste Gashydrate II. Structur und Raumchemie. $Z$. Electrochem. 58, 25-39.

Wei, Xing; Miranda, P.B.; Shen, Y. R. (2001). Surface vibrational spectroscopic study of surface melting of ice. Physical Review Letters, 86, No 8, 1554 - 1557.

Wettlaufer, J. S.; Worster, M. G.; Wilen, L. A. (1997). Premelting dynamics: geometry and interactions. J. Phys. Chem. B, 101, 6137-6141. 


\section{Chapter 3}

\section{Experimental Methods}

\subsection{Neutron Powder Diffraction Method}

The special thermodynamic conditions of gas hydrate stability (high gas pressure/low temperature) complicate in situ studies of the gas hydrate kinetics. The ability of neutron diffraction to work in a complex sample environment, with a time resolution of few seconds, makes it the best choice of experimental technique for following in situ the gas hydrate growth.

\subsubsection{Neutron Diffraction and Method Description}

Neutron diffraction phenomena can be described using the main characteristics of a neutron and its interaction with crystalline matter. The neutron has a zero electric charge and a spin of 1/2. The neutron rest mass of about $1.67 \times 10^{-27} \mathrm{~kg}$ is much higher in comparison to the electron rest mass of about $9.11 \times 10^{-31}$ (Walker, 1995). According to the corpuscular-wave theory the neutron can be described as a classical particle with a mass $m$ and velocity $v$, as well as a wave with a de Broglie wavelength $\lambda=\frac{h}{m v}$. The constant $h$ is Planck's constant. The wave vector $\vec{k}=\frac{2 \pi}{\lambda}$ and the momentum $\vec{p}=h \vec{k}$ are derived from these basic quantities. The relation between the kinetic energy of the neutron and its wavelength can be written as: $E=\frac{m v^{2}}{2}=\frac{h^{2}}{2 m \lambda^{2}}$. According to their kinetic energy, the neutrons can be classified as follows (Table 1): 


\begin{tabular}{|c|c|}
\hline Neutrons & Energy range \\
\hline Thermal & $\sim 0.025 \mathrm{eV}$ \\
\hline Epithermal & $0.025 \mathrm{eV}-100 \mathrm{eV}$ \\
\hline Intermediate & $100 \mathrm{eV}-20 \mathrm{keV}$ \\
\hline Fast & $20 \mathrm{keV}-10 \mathrm{MeV}$ \\
\hline Ultra fast & $>10 \mathrm{MeV}$ \\
\hline
\end{tabular}

Table 1. A free neutron is attributed to the different groups according to its energy. The classification is taken from Walker, 1995.

The nuclear force of interaction between neutrons and atomic nuclei has an extremely short range and an interaction occurs when a neutron almost directly hits a nucleus (Fig. 1), which causes a change of the neutron's wave vector.

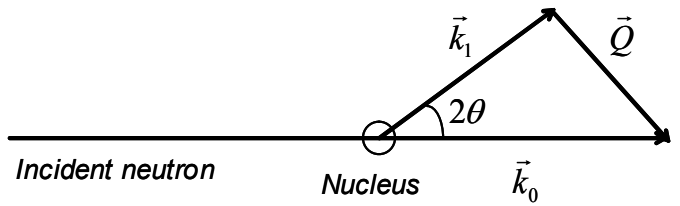

Figure 1. Wave vector transfer $\vec{Q}=\vec{k}_{1}-\vec{k}_{0}$ in a neutron nucleus scattering event. $\vec{k}_{0}$ and $\vec{k}_{1}$ are wave vectors of an incident and a scattered neutron, respectively.

Since the atomic nuclei are much smaller than the atoms, the probability that neutron hits a nucleus is very small and consequently the neutrons can penetrate deeply in the matter before being stopped or scattered. The neutron diffraction method uses an elastic scattering of thermal neutrons at which no energy is transferred $\left(\left|\vec{k}_{1}\right|=\left|\vec{k}_{0}\right|\right.$, Fig. 1). The thermal neutrons can be produced in nuclear reactors where fast neutrons loose their energy in a moderator of heavy water or graphite. Then a monochromator selects a desired wavelength $\lambda$.

The thermal neutrons have a wavelength of $\sim 10^{-10} \mathrm{~m}$, which is comparable to the interatomic distances. The nuclear radius $\left(\sim 10^{-17} \mathrm{~m}\right)$ and the range of the neutron-nucleus interactions $\left(\sim 10^{-15} \mathrm{~m}\right)$ are several times smaller than the wavelength of the incident neutrons. Consequently the atomic nuclei behave as point scatterers with respect to the incident neutron beam.

When an incident beam of neutrons reaches a crystal lattice coherent elastic scattering can occur. An interference image of a crystal can be obtained because the wavelength of incident 
neutrons has the same order of magnitude as the distances between the crystal planes. Bragg's law gives a condition for diffracted beams of maximum intensity to occur in those directions in which a constructive interference takes place between the neutrons scattered by successive layers of atomic planes. A simplified drawing of two such planes satisfying Bragg's equation is given in Fig. 2.

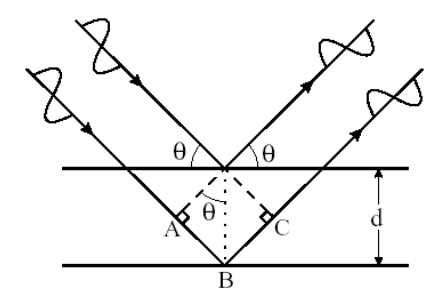

Figure 2. Layout of two planes satisfying Bragg's law.

If $d$ is the interplanar distance and $\theta$ is the angle between the incident and diffracted beams and the planes, the waves scattered by the different planes will have a path difference $A B+B C=2 d \sin \theta$. When it is equal to an integral number of wavelengths: $\mathrm{AB}+\mathrm{BC}=n \lambda$ it gives the Bragg's law: $2 d \sin \theta=n \lambda$.

The diffraction spot, arising from one set of planes in a crystal ((hkl) planes), is called (hkl) reflection. The diffractions, coming from a set of (hkl) planes, randomly oriented in a powder sample, form a diffraction cone with a semi-apex angle $2 \theta$ (Fig. 3a).

a

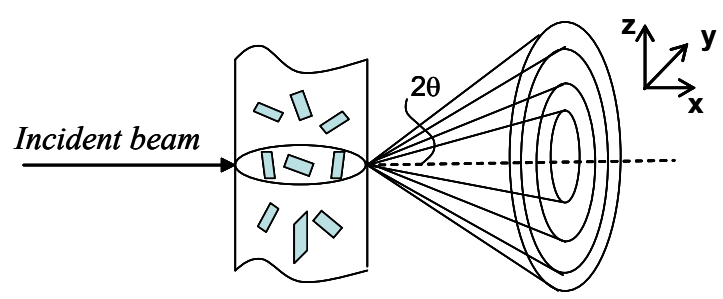

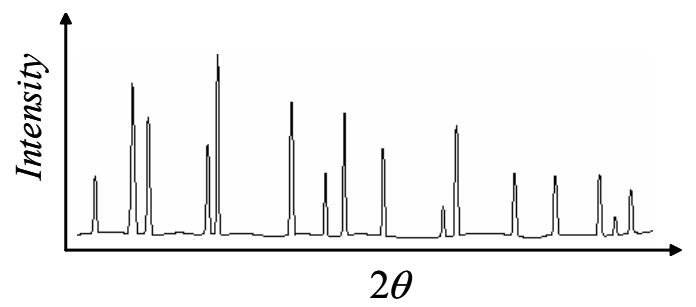

Figure 3. Diffraction of an incident neutron beam from randomly oriented crystallites in a powder sample (a) produces a Bragg-reflections pattern in a one dimensional detector array (b).

The different sets of (hkl) planes make different cones, which are registered as a $2 \theta$ angledispersive powder pattern by one-dimensional detector arrays intercepting the cones (Fig. 3b). 
The diffraction pattern, resulting from the elastic neutron interaction with the crystal lattice, consists of many Bragg reflections. Corresponding to different crystal planes in the sample, they permit in principle a deduction of the crystal structures.

\subsubsection{Rietveld Refinement and GSAS Program}

The need of procedures for extraction of structural information from powder samples led in the late 1960 s to popularization of a computer-based method developed by Rietveld $(1967,1969)$ to analyse powder diffraction patterns by means of a curve-fitting procedure. The method consists of a least-squares refinement, which modifies the input structural model in order to minimize the difference between the observed and calculated profiles. In this sense the Rietveld method is a structure refinement method. The Rietveld method needs a good starting model to calculate a diffraction pattern (Fig. 4) using:

- lattice parameters to calculate the positions of the reflections;

- coordinates and temperature factors of the atoms to determine the intensities of the reflections in an asymmetric unit;

- $\quad$ peak profile functions to model the profile of reflections;

- background functions to model the background.

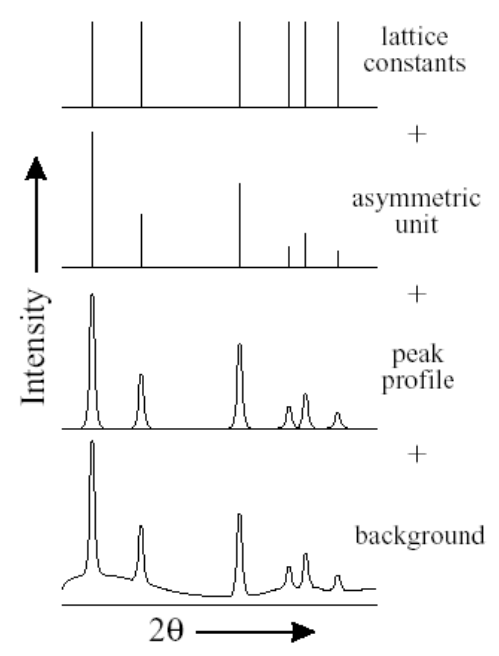

Figure 4. Constructing of a diffraction pattern based on input model information. 
One diffraction pattern can be described numerically by a number of intensity values $y_{i}$ related to a consequence of equal steps $i$, which, for example, can be the scattering angle $2 \theta$. The least-squares refinement minimizes the residual $\Phi_{y}$, calculated as follows:

$$
\Phi_{y}=\sum_{i=1}^{N} w_{i}\left(y_{i}-y_{c i}\right)^{2}
$$

where $w_{i}=\frac{1}{y_{i}}$

$y_{i}$ and $y_{c i}$ are the measured and the calculated intensity at the $i^{\text {th }}$ point, respectively; $N$ is the number of the data points.

As a result of a successive refinement of the model parameters a minimum of the residual (1) is reached and the entire calculated pattern is fitted to the measured pattern. There are a number of criteria of fit, which are helpful to check the quality of the fit. For example $R_{p}=\frac{\sum\left|y_{i o}-y_{i c}\right|}{y_{i o}}$ is a profile $R$-factor, $R_{w p}=\left\{\frac{\sum w_{i}\left(y_{i o}-y_{i c}\right)^{2}}{\sum w_{i} y_{i o}^{2}}\right\}^{1 / 2}$ is a weighted profile $R$-factor, where $y_{i}$ is the intensity at a step $i$, and $w_{i}$ is the related weight factor. The subscripts $o$ and $c$ note the observed and the calculated values, respectively. The progress of the refinement is shown by the weighted profile $R$-factor, $R_{w p}$, which is proportional to the residual (1), being minimised during the refinement. Nevertheless, the quality of the fit, estimated by $R$-factors, has to be always checked by a graphical plot of the data and their fit.

The refinement procedure is controlled by criteria of convergence. The refinement continues until the changes, made in each refined parameter, become smaller than the uncertainty given by an estimated standard deviation. A refinement has reached convergence when during the refinement each parameter stays within its interval of uncertainty. Mathematically this condition is written as:

$$
\sum_{j}\left(\frac{\Delta x_{j}}{\sigma_{j}}\right)^{2}<\varepsilon
$$

where $\Delta x_{j}$ is the change in the parameter $x_{j} ; \sigma_{j}$ is the estimated standard deviation of $x_{j}$; and $\varepsilon$ is the value determining when the refinement will reach a convergence. 
When the convergence is achieved a minimum of the residual (1) is obtained. Figure 5 shows how the residual could change with change of one parameter, for example $x$, which is refined.

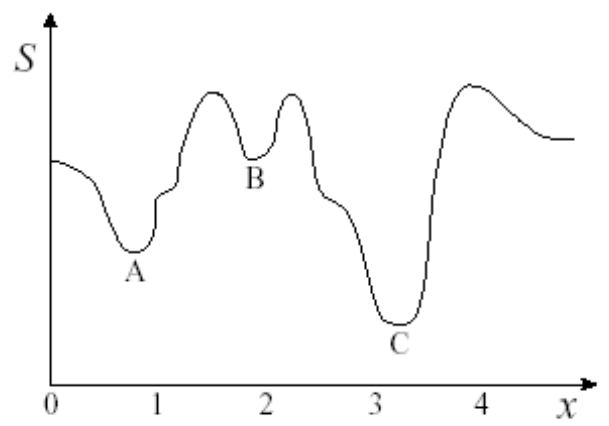

Figure 5. The residual S can reach different minimums (A, B or C) if the refined parameter $x$ has different initial values.

Starting from different values of $x$ (i.e. $0,1.5$ or 3 ) the refinement would lead to different minima of the residual $\mathrm{S}$ at a point $\mathrm{A}, \mathrm{B}$ or $\mathrm{C}$, respectively. The lowest possible value of $\mathrm{S}$ (point $\mathrm{C}$ ) is called a global minimum, which differs from the one at the points A and B, called local (or false) minimum. Therefore it is essential to start the refinement at such parameter's value (for example $x$ $=3$, Fig. 5), which would make the refinement to reach the global minimum. It means the initial model has to describe reasonably well the real crystal structure.

Larson and von Dreele (1990) developed a powerful program package carrying out the Rietveld least-squares refinement of diffraction data. GSAS (Generalized Structure Analysis System) is a multitask software, which has versions running under different operation systems (UNIX, LINUX, Windows) and it is widely used for analyses of powder diffraction data. Kinetic diffraction data of gas hydrate formations were processed using the Rietveld refinement method, implemented in GSAS program, to model the (hkl) reflections, which belong to all crystalline phases in the diffracting sample.

The intensity at any point in a powder diffraction pattern has contributions from nearby reflections and a background scattering. The reflection contributions are calculated from the structure factors and the background is modeled by empirical functions (Larson and von Dreele, 1990). Thus, the calculated intensity $y_{c}$ is determined as:

$$
y_{c}=s_{h} \sum_{p} s_{p h} Y_{p h}+y_{b}
$$


where $s_{h}$ is a histogram scale factor, applied to the reflections from all phases in the sample; $s_{p h}$ is an individual phase scale factor, applied only to the reflections from the $p^{\text {th }}$ phase; $Y_{p h}$ is the contributed intensity from $h^{\text {th }}$ Bragg reflection of the $p^{\text {th }}$ phase; $y_{b}$ is the background value.

The contributed intensity $Y_{p h}$ from a Bragg peak to particular profile intensity is determined as:

$$
Y_{p h}=F_{p h}^{2} \phi\left(\theta-\theta_{p h}\right) K_{p h}
$$

where $F_{p h}$ is the structure factor for a particular reflection; $\phi\left(\theta-\theta_{p h}\right)$ is the value of a profile peak shape function for that reflection at position $\theta$, displaced from its expected position $\theta_{p h}$; $K_{p h}$ is an intensity correction factor for that reflection. Each scale factor $s_{p h}$ is proportional to a number of unit cells of the related phase that is present in the sample. The scale factor can be converted to a weight fraction $W_{p}$ of a phase in a multiphase composition:

$$
W_{p}=\frac{s_{p h} m_{p}}{\sum_{p=1}^{N_{p}} s_{p h} m_{p}}
$$

where $m_{p}$ is a unit cell mass for a phase $p$, computed from the atom site multiplicities and fractions, which are present for that phase. The weight fractions for multiphase mixtures are automatically computed during the least-squares refinement in GSAS (program GENLES) and are given together with their estimated standard deviations in an output ASCII file.

\subsubsection{Neutron Diffraction Instrument}

In situ kinetic experiments on the gas hydrate formation were performed using the neutron powder diffraction method at High-Intensity 2-Axis Neutron Diffractometer D20 (Figure 6), Institute Laue-Langevin (ILL) in Grenoble, France. A description of D20 is available at http://www.ill.fr/YellowBook/D20 and in the papers of Convert et al. $(1998,1999)$. 


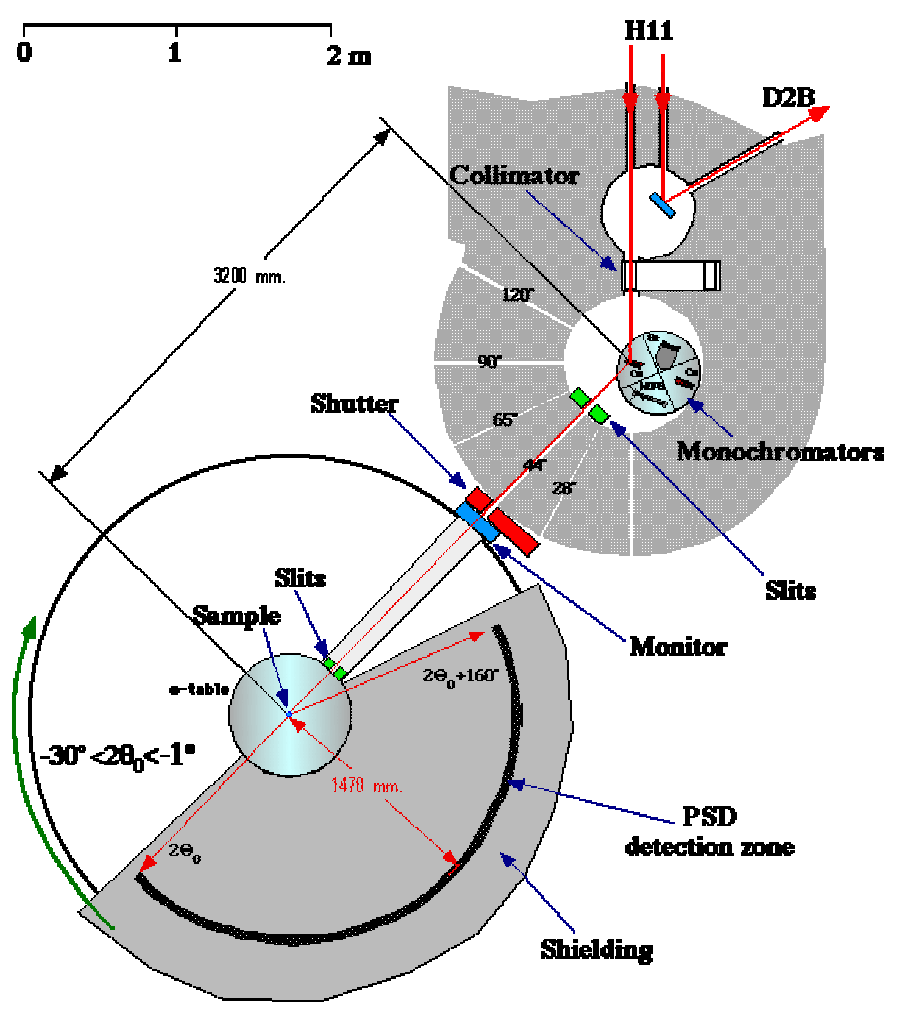

Figure 6. Schematic drawing of the D20 set-up.

At it is shown with the geometry of the measurement a monochromatic neutron beam reaches the sample located at $3200 \mathrm{~mm}$ from the monochromator. The neutrons, diffracted by the sample, are registered by a large-area Position Sensitive Detector. It covers a part of a circle with a centre in the sample position.

D20 uses a monochromatic thermal neutron beam with a very high flux. A monochromator of pyrolitic graphite HOPG (002) in reflection position with fixed vertical focusing, giving a wavelength of $2.4 \AA$ at a take-off angle of $42^{\circ}$, was chosen to carry out the kinetic experiments presented in this work. The beam flux, coming after the monochromator, is about $4.2 \cdot 10^{7} \mathrm{n} \cdot \mathrm{cm}^{-2} \cdot \mathrm{s}^{-1}$.

The Position Sensitive Detector (PSD), used in D20, provides a detection zone of about 4 $\mathrm{m}$ long and $150 \mathrm{~mm}$ high. The PSD is filled with 3.1 bar ${ }^{3} \mathrm{He}$ and 0.8 bar $\mathrm{CF}_{4}$. This large PSD is made by using of a specially developed micro-strip gas chamber (MSGC) technology. It consists of sputtered chromium on a polished surface of electronically conducting glass plates. Then the chromium is etched to create conductive micro-strip electrodes (alternately 4 cathodes and 4 thin anodes per detection cell). The small distance between the anode and cathode $(170 \mu \mathrm{m})$ provides 
fast evacuation of the positive ions, and allows very high counting rates. Each detection plate, consisting of 32 cells, covers $3.2^{\circ}\left(0.1^{\circ}\right.$ per cell). The PSD covers $153.6^{\circ}$ with 48 plates mounted on a distance of $1471 \mathrm{~mm}$ from the sample. The 32 cells of one plate have 32 independent outputs from the detector through a metal-ceramic plug. All 1536 cells are connected to an identical number of amplifiers, followed by an identical number of anti-coincidence logic units. After the amplifier signal first passed the discriminator threshold for a particular cell, its neighboring cells are prevented from counting the same event second or third time during $1.5 \mu \mathrm{s} ; 2.5 \mu \mathrm{s}$ after having passed the threshold a cell may count again an event. The limit of the counting rate is about $50000 \mathrm{~s}^{-1}$ per cell. This micro-strip detection system has a precise and very stable geometry.

D20 is equipped also with a data acquisition system (DAS), which has a parallel input for up to 1600 cells. The instrument allows for measuring of a series of short diagrams, each lasting typically few seconds, to observe irreversible kinetic phenomena. In addition D20 is equipped with a Silicon Graphics workstation for an instrument and an acquisition control and with a large array manipulation program - LAMP (Richard et al.) - for an efficiency correction and a two- and three-dimensional plot of the collected data.

The high flux at the sample position, the large stationary PSD and the fast DAS make D20 an ideal tool for the purposes of studying the gas hydrate kinetics.

\subsubsection{Pressure and Temperature Control}

High pressure/low temperature conditions, providing the process of gas hydrate formation, required the use of high pressure and cryogenic equipment (see Fig. 7). High pressure at the sample position was obtained by equipment built in Göttingen. It consisted of high-pressure lines, valves and a piezoelectric pressure sensor ASHCROFT Type KXD of a range (0-60 bar) with accuracy of $0.25 \%$. A pressure regulator (Fig.7) of type FM61 (Messer Cutting \& Welding $\mathrm{GmbH}$ ) was used to reduce the inlet pressure, coming from the gas bottle, to the desired outlet pressure and to keep it at a constant value. 


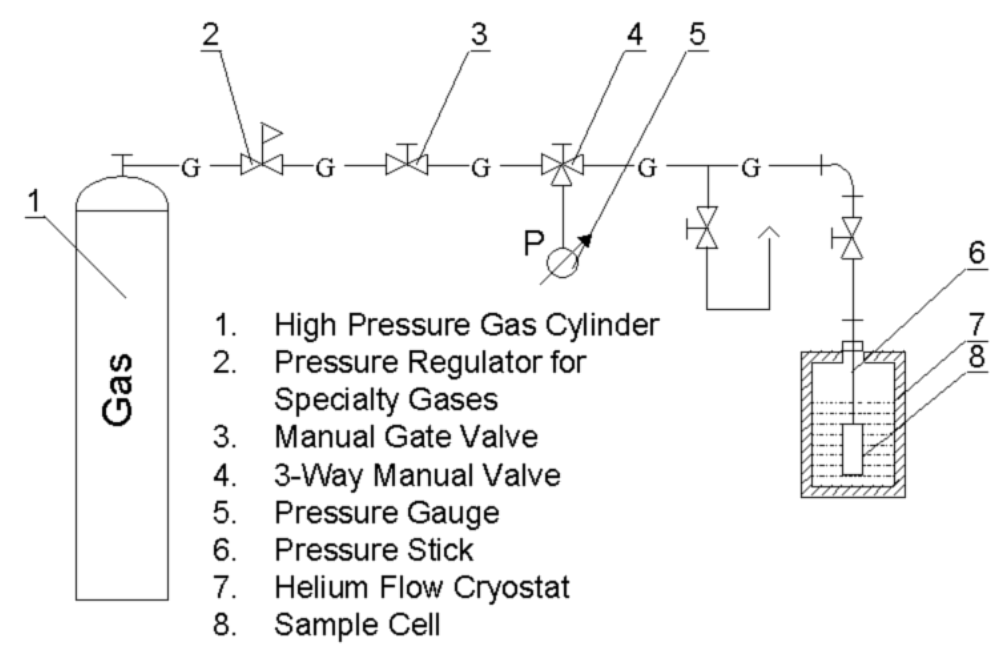

Figure 7. Scheme of the high-pressure set-up used for the in situ kinetic experiments at D20 (provided by Georgi Genov-GZG, Göttingen).

The sample temperature reading (Fig. 8) was obtained from a calibrated temperature sensor fixed to the wall of the pressure cell. In addition, the pressure stick was equipped with a capillary heating coil, controlling the temperature of the capillary close to the one of the reaction. The aluminium gas pressure cells used in the neutron diffraction experiments were manufactured at GZG, Göttingen (Fig. 9). 
1. Sample temperature control socket

2. Capillary heating socket

3. Stick height adjusting unit

4. Helium flow cryostat

5. Centering plugs

6. Capillary heating (COWAX)

7. Gas capillary

8. Pressure cell

9. Bridgman seal

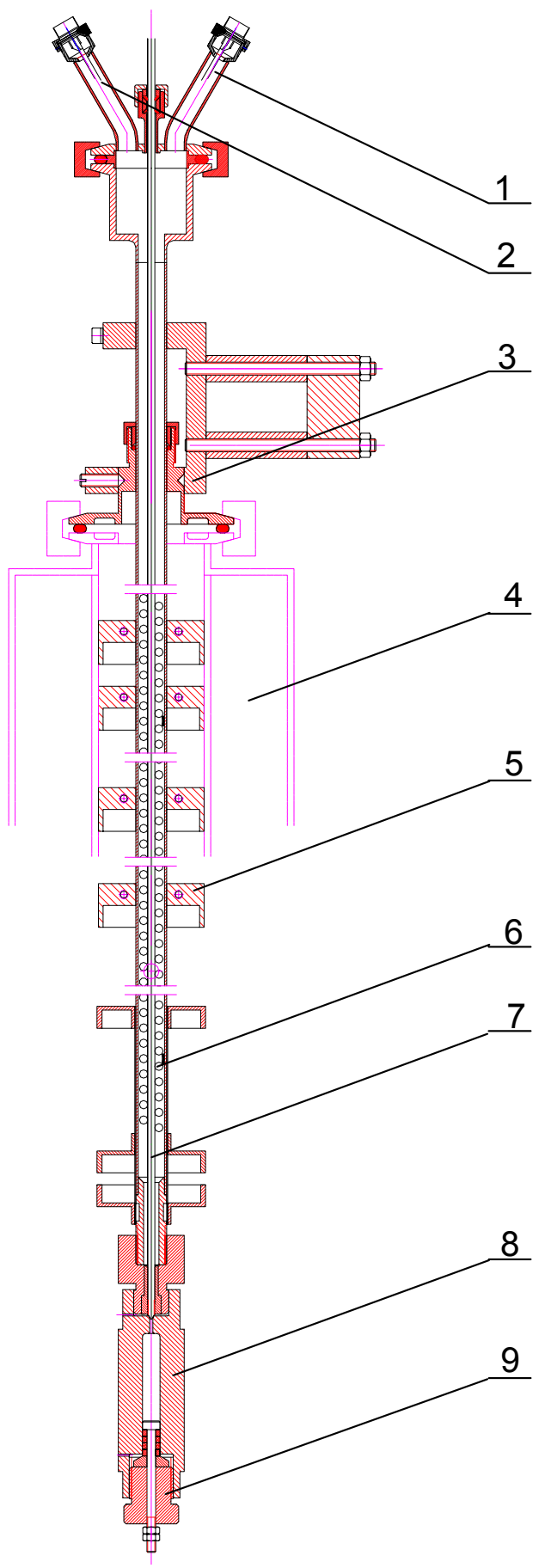

Figure 8. Scheme of the pressure stick used at ILL-Grenoble (provided by Klaus Haepe-GZG, Göttingen). 


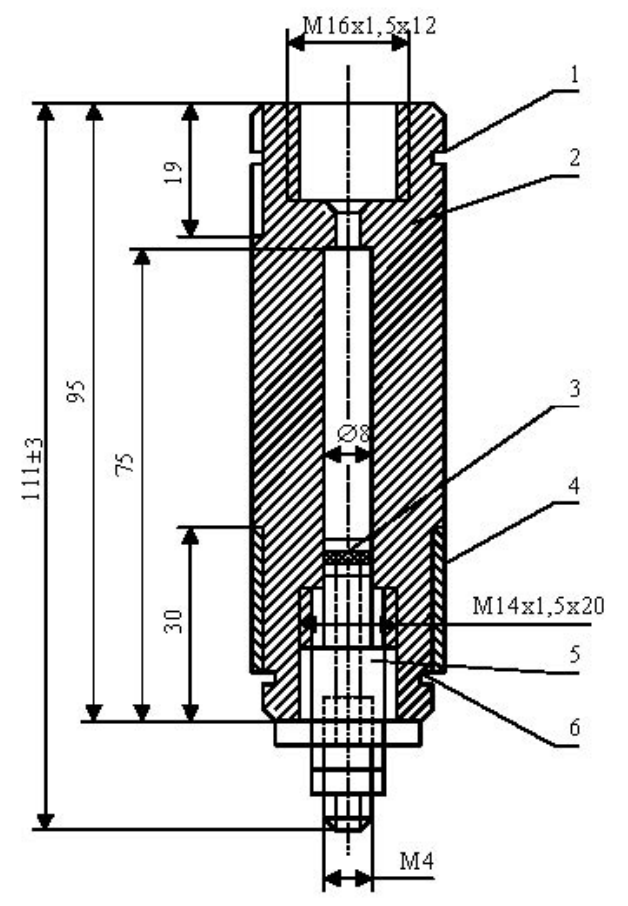

1. Spring lock washer SW 22

2. Aluminum corps

3. Bridgman seal

4. Cadmium cylinder $(0,5 \mathrm{~mm}$ thick)

5. Sealing device

6. Spring lock washer SW 20

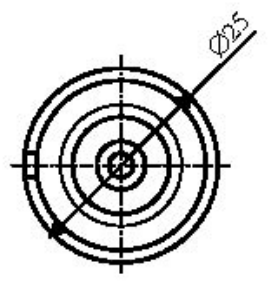

Figure 9. Scheme of the pressure cell used in the neutron diffraction experiments (provided by Georgi Genov GZG, Göttingen).

The figure shows the pressure cell with its cadmium cylindrical shield $\left(\mathrm{N}^{\circ} 4\right)$ made to prevent the Bridgman seal from irradiation and to prevent parasitic scattering into the detector.

The temperature control during the in situ kinetic experiments was provided by a Helium Flow Cryostat ("Orange cryostat") specially modified for D20 (Fig. 10.1-10.2). The pressure cell, connected to the pressure stick, was placed in a chamber with only 10-20 mbar of helium exchange gas. It made the thermal contact between the cell and the surrounding sample chamber. The chamber temperature was controlled by opening the "cold" valve, which changed the helium gas flow through the chamber. The fine temperature control was done by the heating coil of the heat exchanger. In addition a thermocouple was placed at the sample-chamber wall to provide a 
reference temperature control. The DTI temperature control interface adjusts automatically the cryostat temperature with precision of a fraction of the degree, using the previously mentioned reference temperature.

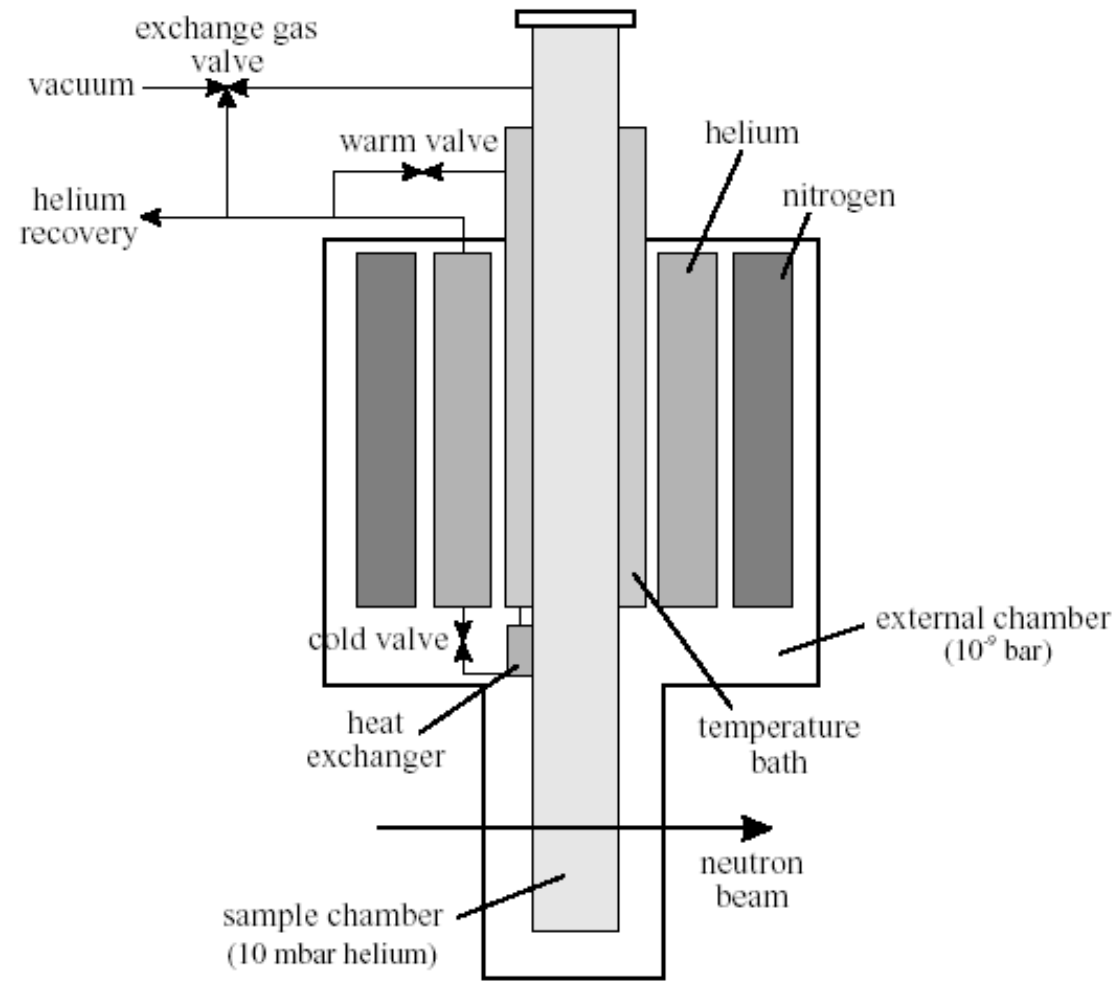

Figure 10.1. Scheme of helium flow cryostat (“Orange cryostat”) (provided by Klaus Haepe-GZG, Göttingen).

Figure 10.2 shows a plane and cross-section drawing of the D20 cryostat. It is designed to reduce the detected background produced by the interaction between the high-intensity neutron beam and the cryostat walls. 


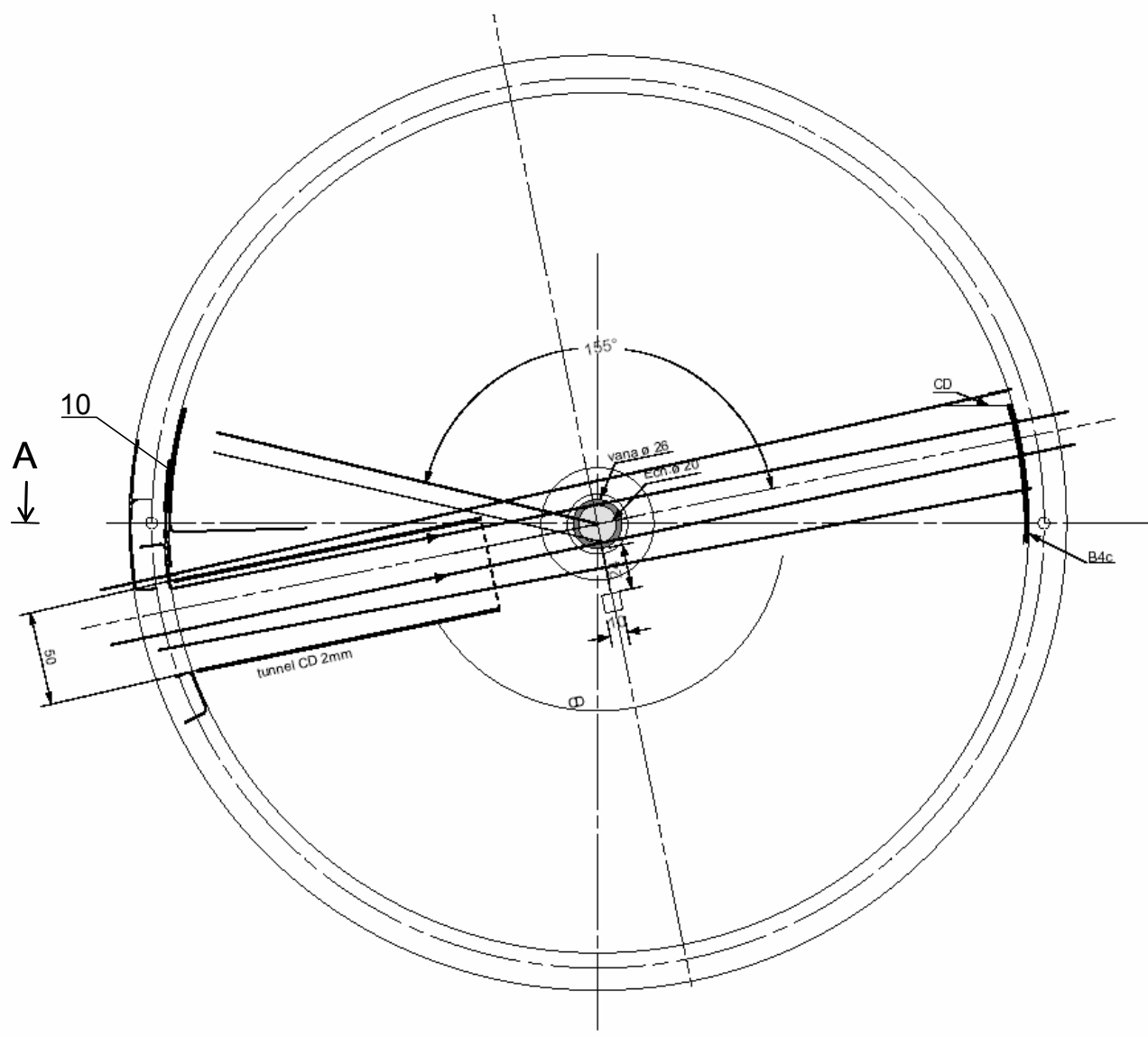

A

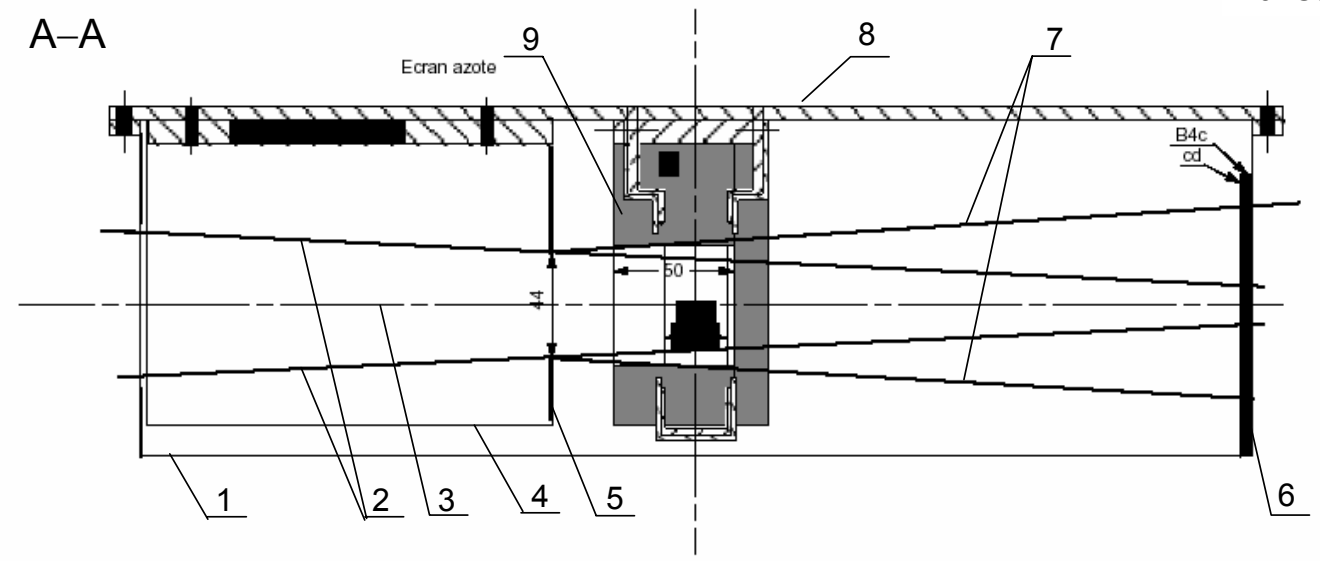

1. Al shielding at room $T$

2. Total neutron beam

3. Centre of the beam

4. Cadmium tunnel

5. Cadmium window

6. Beam stop

7. Divergent beam

8. Al shielding at $\mathrm{T}$ of liquid $\mathrm{N}_{2}$

9. Vanadium calorimeter

10. Cadmium masking

Collimation cryo. d20 Ech. $1 / 2$

Figure 10.2. Drawings of the cryostat located at D20-ILL (provided by Thomas C. Hansen-D2O, ILLGrenoble). 


\subsubsection{Ice Powder Starting Material}

An ice Ih powder was used as a starting material for the in situ neutron diffraction studies of the gas hydrate formation. The interaction of the neutrons with the isotopes, hydrogen and deuterium, is different. The coherent neutron scattering gives the wanted diffraction pattern of a crystalline phase. The high cross-section of an incoherent scattering of the hydrogen (Table 2) produces a large background in the neutron diffraction pattern.

\begin{tabular}{|c|c|c|}
\hline Symb & $\sigma_{\text {coh }}$ & $\sigma_{\text {inc }}$ \\
\hline $\mathrm{H}$ & $1.7583(10)$ & $80.27(6)$ \\
\hline $\mathrm{D}$ & $5.592(7)$ & $2.05(3)$ \\
\hline
\end{tabular}

Table 2. Scattering cross-sections of two hydrogen isotopes: protium $\mathbf{H}$ and deuterium $\mathbf{D}$. Symb is the element symbol, $\sigma_{\text {coh }}$ - coherent cross section in barn and $\sigma_{\text {inc }}$ - incoherent cross section in barn. The values are taken from Dianoux and Lander (2002).

As can be seen from Table 2 this makes the peak-to-background ratio for the hydrogen very small. Thus, a deuterated sample is preferred for the studies of powders. The present work shows mainly results of in situ kinetic studies of the gas hydrate formation from $\mathrm{D}_{2} \mathrm{O}$ ice. A sample holder, specially designed to decrease the incoherent scattering in the sample by a decrease of its volume (see below), was used to attempt an in situ hydrate formation from $\mathrm{H}_{2} \mathrm{O}$ ice.

The starting material was produced in the physico-chemical laboratory at GZG-Göttingen, using a spraying/shock-freezing method. The ice Ih powder was made by a spraying of water in liquid nitrogen. Accidentally produced grains, bigger than $200 \mu \mathrm{m}$, were removed by a sieving of the material. The remaining powder was collected and filled into thin-walled Al-cans (Fig. 11a) with a measured packing density of $65-70 \%$. The sample of $\mathrm{H}_{2} \mathrm{O}$ ice powder used in the neutron diffraction experiments was filled in the Al cans with placed small Al inserts (Fig. 11b). 
a
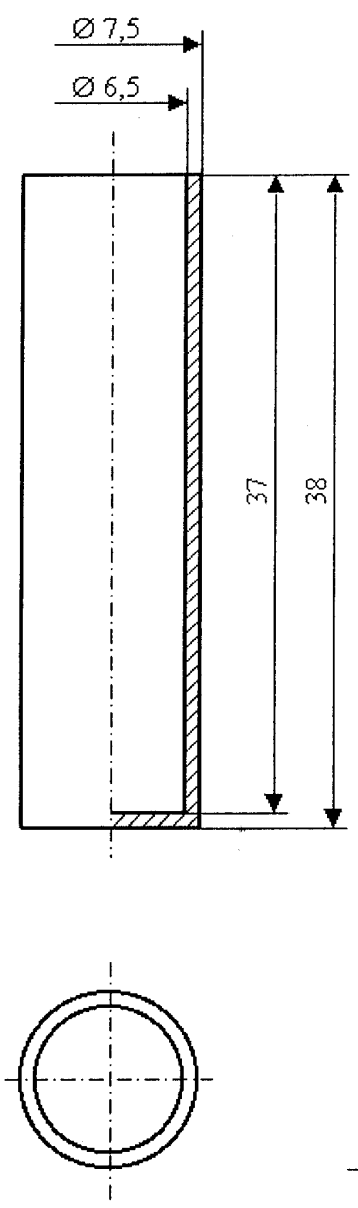

b
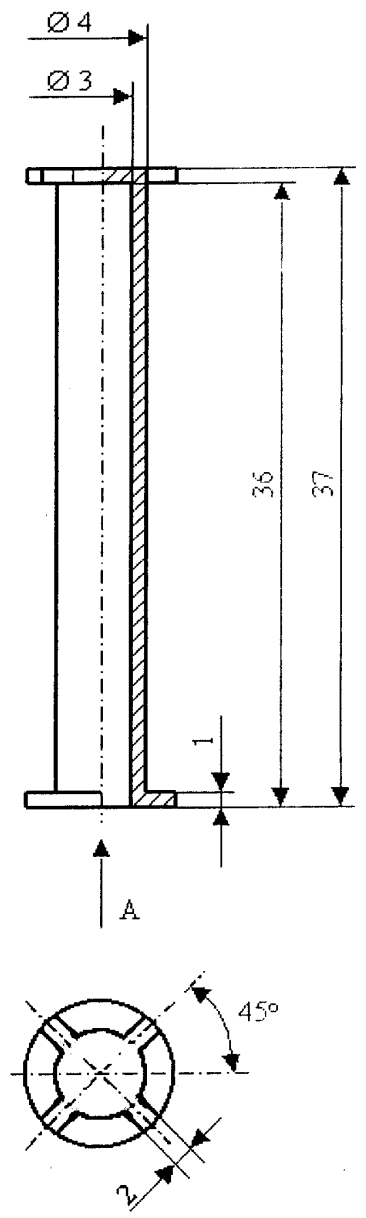

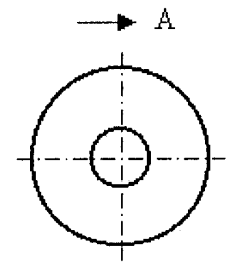

Figure 11. Schematic draw of the Al can used as a sample holder for the kinetic studies of the gas hydrate formation (a). For the neutron kinetic experiments each hydrogenated sample was filled in a can with an Al insert in it (b). Drawings are provided by Georgi Genov, GZG-Göttingen.

For the experiments No. 2-4 (Table 3, Ch. 3.1.6) the prepared sample was annealed for $3 \mathrm{~h}$ in a fridge at temperature of $-28^{\circ} \mathrm{C}$ in order to anneal the stacking faults in the ice Ih introduced by the production method of the spheres. In order to quantify the morphology of the starting material a representative part of the sample was investigated by a cryo field-emission scanning electron microscopy (FE-SEM). The obtained pictures were used to estimate a size distribution of the ice spheres produced by the spraying technique. Measurements on different batches showed that the size distribution of the ice spheres sprayed with the same nozzle was well reproducible and had a 
lognormal shape. Details about the FE-SEM method, obtained pictures and their statistical processing are described in Chapter 3.4.4. Samples with different particle size can be produced using different nozzles. For example, in the four series of experiments, described in Chapter 3.1.6, the mean particle diameter was about 70 and $54 \mu \mathrm{m}$.

\subsubsection{Experiments Description}

The Al-cans with the prepared ice samples were transported in a dry- $\mathrm{N}_{2}$ portable Dewar to ILL/Grenoble. The pressure cell, already fixed to the sample stick (Fig. 9), was cooled by dry ice. The Al cans were inserted into the pressure cell at an applied small stream of gas to ensure a complete filling of the system with the selected gas. Than the Bridgman seal was closed. The pressure stick was inserted into the "Orange cryostat" and the temperature was equilibrated at a chosen value. After the temperature was adjusted, high gas pressure was applied within a few seconds while the data collection was started concomitantly. According to the security rules at the instrument the high-pressure gas bottle should be closed if there was nobody at the experiment. This was not a problem because the pressure lines of large volume supplied the sample with high gas pressure of almost constant value during the runs. Only from time to time, when the pressure decreased with a fraction of the bar, it was readjusted manually.

Series of in situ neutron diffraction experiments were performed to study the kinetics of gas hydrate formation from deuterated and hydrogenated ice (see Table 3). The reaction runs usually lasted 8-23h. Two long intermittent runs covering a reaction time of 5-6 days were performed as follows. Each reaction was started in the "Orange cryostat" and recorded for about 20h. Then the data acquisition was stopped and the reaction was continued out of the beam for a few days in a bath adjusted to the temperature of the reaction. Within this period each stick was taken twice back into the "Orange cryostat" and the reaction degree was measured - once in the middle of the reaction time and then at the end.

The mean radius of the ice grains in the first set of experiments was larger $(35 \mu \mathrm{m})$ than in the other ones $(27 \mu \mathrm{m})$. The thermodynamic conditions and the duration of the observed runs are presented in Table 3. 


\begin{tabular}{|c|c|c|c|c|c|c|c|c|}
\hline \multirow{2}{*}{$\begin{array}{c}\text { Series } \\
\text { No. }\end{array}$} & \multicolumn{8}{|c|}{ Conditions of experiments } \\
\hline & $\begin{array}{l}r_{i 0}, \\
\mu \mathrm{m}\end{array}$ & Ice & Gas & $\begin{array}{l}T, \\
\mathrm{~K}\end{array}$ & $\begin{array}{l}P(f) \\
\mathrm{MPa}\end{array}$ & $\begin{array}{c}P_{d}\left(f_{d}\right), \\
\mathrm{MPa}\end{array}$ & $\begin{array}{c}\text { Excess } \\
\text { fugacity, } \\
\left(f-f_{d}\right) / f_{d}\end{array}$ & $\begin{array}{c}\text { Duration, } \\
\mathrm{h}\end{array}$ \\
\hline \multirow{3}{*}{1} & \multirow{3}{*}{35} & \multirow{3}{*}{$\mathrm{D}_{2} \mathrm{O}$} & $\mathrm{CO}_{2}$ & \multirow{3}{*}{272} & $2.00(1.73)$ & $1.08(1.00)$ & 0.73 & 18 \\
\hline & & & & & $3.50(3.22)$ & $2.50(2.36)$ & 0.36 & 23 \\
\hline & & & $\mathrm{CH}_{2}$ & & $6.00(5.20)$ & $2.50(2.36)$ & 1.2 & 18 \\
\hline \multirow{4}{*}{2} & \multirow{4}{*}{27} & \multirow{4}{*}{$\mathrm{D}_{2} \mathrm{O}$} & \multirow{4}{*}{$\mathrm{CH}_{4}$} & 268 & $6.00(5.16)$ & $2.19(2.07)$ & 1.49 & 8 \\
\hline & & & & 230 & $3.50(3.02)$ & $0.59(0.58)$ & 4.21 & 11.5 \\
\hline & & & & 230 & $6.00(4.62)$ & $0.59(0.58)$ & 6.97 & 11 \\
\hline & & & & 272 & $6.00(5.20)$ & $2.50(2.36)$ & 1.2 & 1.5 \\
\hline \multirow[t]{2}{*}{3} & \multirow[t]{2}{*}{27} & \multirow[t]{2}{*}{$\mathrm{D}_{2} \mathrm{O}$} & \multirow[t]{2}{*}{$\mathrm{CH}_{2}$} & 270 & $6.00(5.19)$ & $2.33(2.20)$ & 1.36 & $135.4^{*}$ \\
\hline & & & & 263 & $6.00(5.11)$ & $1.84(1.75)$ & 1.94 & $104.5^{*}$ \\
\hline \multirow{3}{*}{4} & \multirow{3}{*}{27} & \multirow{3}{*}{$\mathrm{H}_{2} \mathrm{O}$} & \multirow{3}{*}{$\mathrm{CH}_{4}$} & 264 & $6.00(5.12)$ & $1.90(1.81)$ & 1.83 & 17.5 \\
\hline & & & & 253 & $6.00(4.99)$ & $1.34(1.29)$ & 2.87 & 23 \\
\hline & & & & 230 & $6.00(4.62)$ & $0.59(0.58)$ & 6.97 & 14 \\
\hline
\end{tabular}

* total period of interrupted runs

Table 3. Experimental p-T conditions of $\mathrm{CO}_{2}$ and $\mathrm{CH}_{4}$ gas hydrate formations studied in situ by the neutron diffraction method.

Each experiment was done at a certain position in the field of thermodynamic stability of the gas hydrate phase. This location can be described in an absolute scale (as a position (MPa, K) in the phase diagram (Fig. 11-12, Ch. 2)), as well as in a scale with respect to the gas hydrate decomposition curve (as an excess fugacity). Both descriptions are shown in the table. The values of the decomposition pressure $P_{d}$ of $\mathrm{CH}_{4}$-gas hydrate at those temperatures were calculated as follows:

- for the temperatures up to $265 \mathrm{~K}$ using equation (6) (see Appendix A.1)

$$
P_{d}=\exp (8.448-2064.4080 / T)
$$

- for the temperatures between $265 \mathrm{~K}$ and $273.15 \mathrm{~K}$ using linear approximations between the literature values given by Sloan (1998) for this temperature interval.

The decomposition pressure of $\mathrm{CO}_{2}$-gas hydrate at temperature of $272.15 \mathrm{~K}$ was obtained using the program CSMHYD.EXE (Sloan, 1998).

Fugacity values at the experimental thermodynamic conditions were obtained from fugacity/pressure ratios calculated as linear approximations of the closest available values taken from the International Thermodynamic Tables (1973 and 1976) (Eq. 1, Appendix A.2). They 
were used to calculate the parameter of excess fugacity $\frac{f-f_{d}}{f_{d}}$ used in the data interpretation (Ch. 5).

\subsubsection{Data Collection}

Diffraction patterns were collected during the gas hydrate kinetic runs, described above, using D20 at its highest intensity setting, at $\lambda=2.414 \AA$. The gas and water molecules in the ice Ih - gas system start to form new clathrate phase at the thermodynamic conditions of its stability. The gas hydrate growth was observed as an increase of the Bragg intensities originating from the hydrate phase while the Bragg intensities of ice Ih decreased. The clathrate structure started to form immediately after high gas pressure was applied. Each reaction of the gas (at constant pressure and temperature) with the ice grains was followed continuously over a period of typically 10 to $20 \mathrm{~h}$. One set of powder diffraction data, recorded during a reaction of methane hydrate growth, is shown with an example plot of D20 program LAMP (Fig. 12). It illustrates the most relevant appearing Bragg reflections of the clathrate phase and their intensities increase with the time.

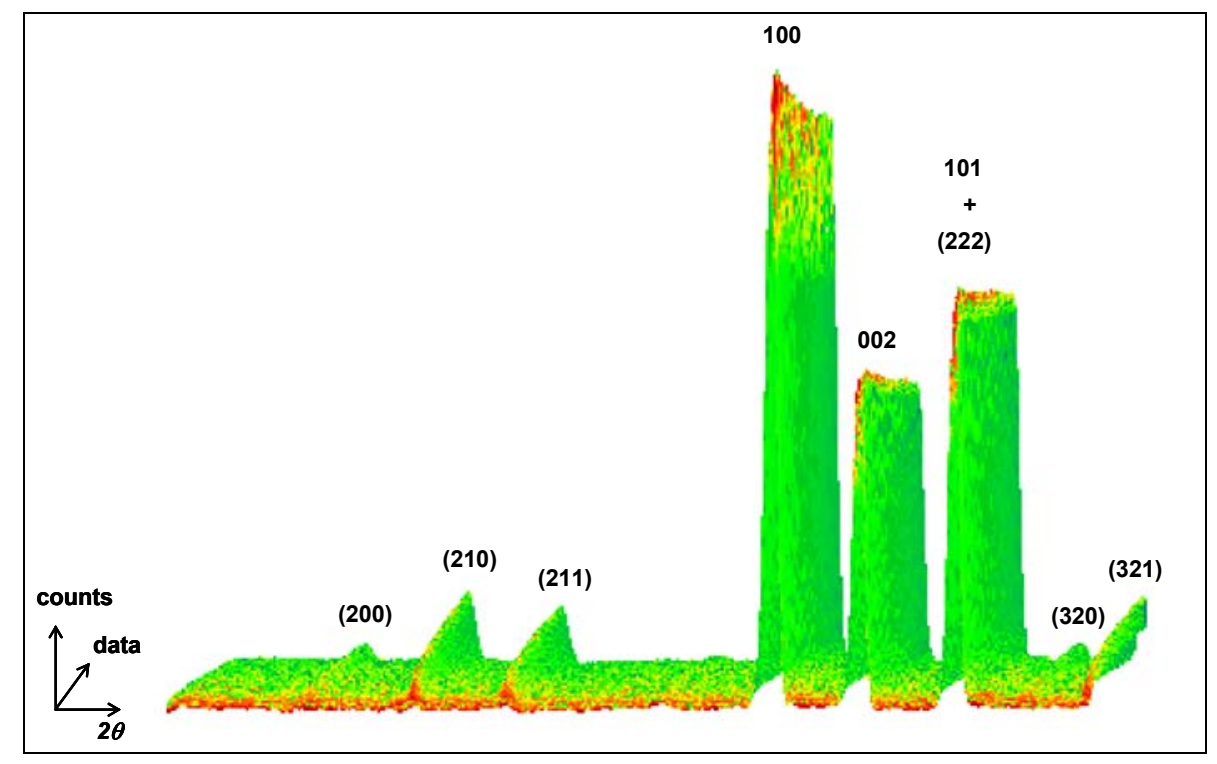

Figure 12. A LAMP plot of one set of neutron diffraction data collected during an in situ kinetic run: 2 $\theta$ region covering the most intense Bragg reflections of Ice Ih and the growing $\mathrm{CH}_{4}$-hydrate crystal structure $\mathrm{I}$. The (hkl) Miller indices of the Bragg reflections shown are marked with numbers with and without brackets for type-I and ice Ih, respectively. The followed reaction was $\mathrm{CH}_{4}-\mathrm{D}_{2} \mathrm{O}$ gas hydrate formation for $24 \mathrm{~h}$ at temperature of $-10^{\circ} \mathrm{C}$ and gas pressure of $6.0 \mathrm{MPa}$. 
The neutron diffraction data were collected with a time resolution of $30 \mathrm{~s}$ or $1 \mathrm{~min}$ for the initial fast reaction and with a resolution of 5 min for the slower later part of the reaction. Data of very good statistical precision were obtained suggesting that even time slices of $10 \mathrm{~s}$ would deliver useful information. It was observed that all gas hydrate reactions started immediately (within the diffractometer time resolution of a few seconds) after applying gas pressure higher than the decomposition one, i.e. no induction time was found. After an efficiency correction the data were transformed to GSAS format and transferred to a Linux PC in Göttingen.

\subsubsection{Data Analyses}

The Rietveld refinement method realized by GSAS program (Ch. 3.1.2) was used to analyse the collected neutron diffraction data. It was found that some of the raw data of GSAS format had an error in the header (see Fig. 13). It occurred when they were produced from the raw experimental data by the LAMP program at the instrument.

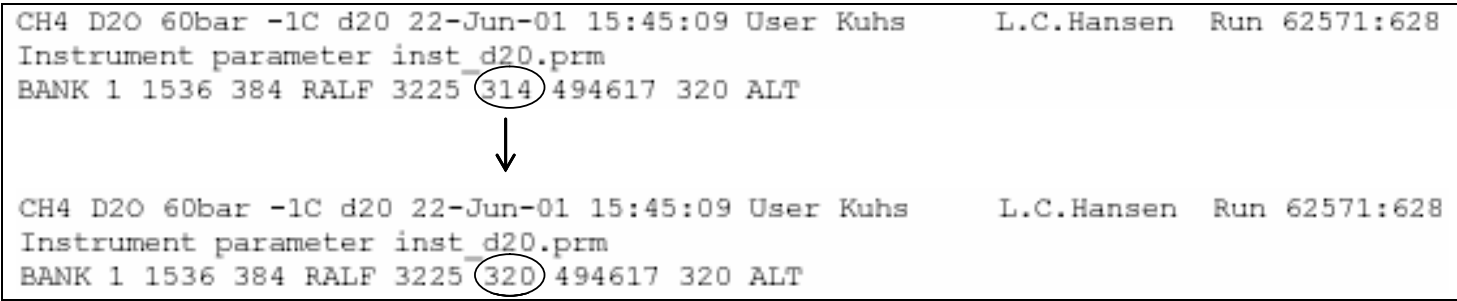

Figure 13. Example of a found error in the headers of the neutron diffraction data of GSAS format which did not allow the data processing before its repair. The number surrounded by a circle (above) varied in the range of 300-900 for the different data files. It had to be replaced by a value in the circle (below) to permit a data treatment (see the text).

The input powder data file used in the GSAS program has a header depending on the type of the following data. For a standard type of data the header is:

('BANK', 3I, A, 4F, A) IBANK, NCHAN, NREC, BINTYPE, (BCOEF (I), I=1, 4), TYPE

where IBANK is a bank number (e.g. 1); NCHAN is the number of data points in the block of data (e.g. 1536); NREC is the number of records (e.g. 384 records of 4points/record). The form of header with a TYPE of 'ALT' shows that the data records include the position of each point in somewhat unusual units. A BINTYPE of 'RALF' makes GSAS to use a BCOEF (2) (marked in the circle, Fig. 13) to control the steps between the data points within the data block. An error in the BCOEF (2), which occurred when GSAS powder diffraction data were made, caused problem to 
process the data. When the value of BCOEF (2) was changed (Fig. 13) the neutron diffraction data were processed.

As it was mentioned before each obtained neutron diffraction pattern was analysed by means of a full-pattern least squares Rietveld refinement performed by GSAS program. Using mainly the models deduced by Klapproth (2002), the structure model of the growing gas hydrate phase was introduced for each kinetic run. The structure of type $\mathrm{II} \mathrm{CO}_{2}$-hydrate was built using an existing model of type II $\mathrm{N}_{2}$-hydrate (Chazallon, 1999). The models used in the Rietveld refinement of all neutron diffraction data (Table 3, Ch.3.1.6) are given in Table 1-4, Appendix A.3-A.5. The atomic positions and the displacement parameters of the input models were kept fixed during the refinement of the gas hydrate kinetic data. For each data set the lattice constants of ice Ih and gas hydrate were kept fixed at values, refined from the last recorded diffraction pattern. The background, coming from the incoherent scattering, air scattering, and the flat parts of thermal diffuse scattering was modelled using cosine Fourier series (Larson and Von Dreele, 1990)

$$
y_{b}(Q)=B_{1}+\sum_{j=2}^{N} B_{j} \cos (Q(j-1))
$$

where $B_{j}, j=1 . . N$ are determined by the Rietveld refinement background parameters and $Q$ is a position, in degrees $2 \theta$, along the diffraction profile. This mathematical description of the background can be applied in case of a slowly changing generally flat background. Cosine Fourier series with five parameters were used to describe the background in the collected neutron diffraction data. The reflection profiles were modelled by the GSAS program using a profile peak shape function (No 2) based on the works of Howard (1982) and Thompson at al. (1987).

For each kinetic run an experimental (EXP) file making two-phase (ice Ih + gas hydrate) Rietveld fit was prepared. Examples of a performed full-pattern Rietveld refinement of $\mathrm{CH}_{4}$ - and $\mathrm{CO}_{2}$-hydrate diffraction data are presented in Appendix A.3-A.5 (Fig. 2-4). For each kinetic run the refinement started with the data of the last recorded time slice. Thus, the lattice constants, the profile parameters and the phase fractions of ice Ih and gas hydrate, corresponding to the end of the reaction, were refined as well the background parameters. Once the successive refinement was obtained and convergence was achieved, the lattice constants and the profile parameters were fixed in the EXP file. This file was copied to a backup file (BAC) and used as an 'initial' EXP file (see below). 
All sets of collected data were analysed with a Linux version of GSAS. It was done in an automated way using Linux command lines. The algorithm of the automatic data processing (see Fig.14) included a GSAS refinement of each diffraction pattern by one and the same EXP file ('initial file'). This was necessary to avoid a crash of the automatic procedure if some raw data files create problems in the refinement. Before the start of the automatic refinement the header of each data file was corrected (see Fig.13). Then the corrected file was copied in a buffer file, which was the raw histogram input file of the 'initial' EXP file. After the GSAS program was started a program (EXPNAM) introduced the EXP file for a processing. Then the BAC file, which has the content of the 'initial file', is copied to the EXP file. After GSAS processing (by POWPREF and GENLES programs) an output file (LST) of the results of the Rietveld fit was produced. Then the name of the refined data was written in the same LST file. The procedure described above was repeated in a cycle until all data files were processed. The results of their refinement were collected in one (LST) file. Then the values of the gas hydrate and the ice weight fraction, corresponding to each refined diffraction pattern, were extracted from the LST file using Linux command lines. 


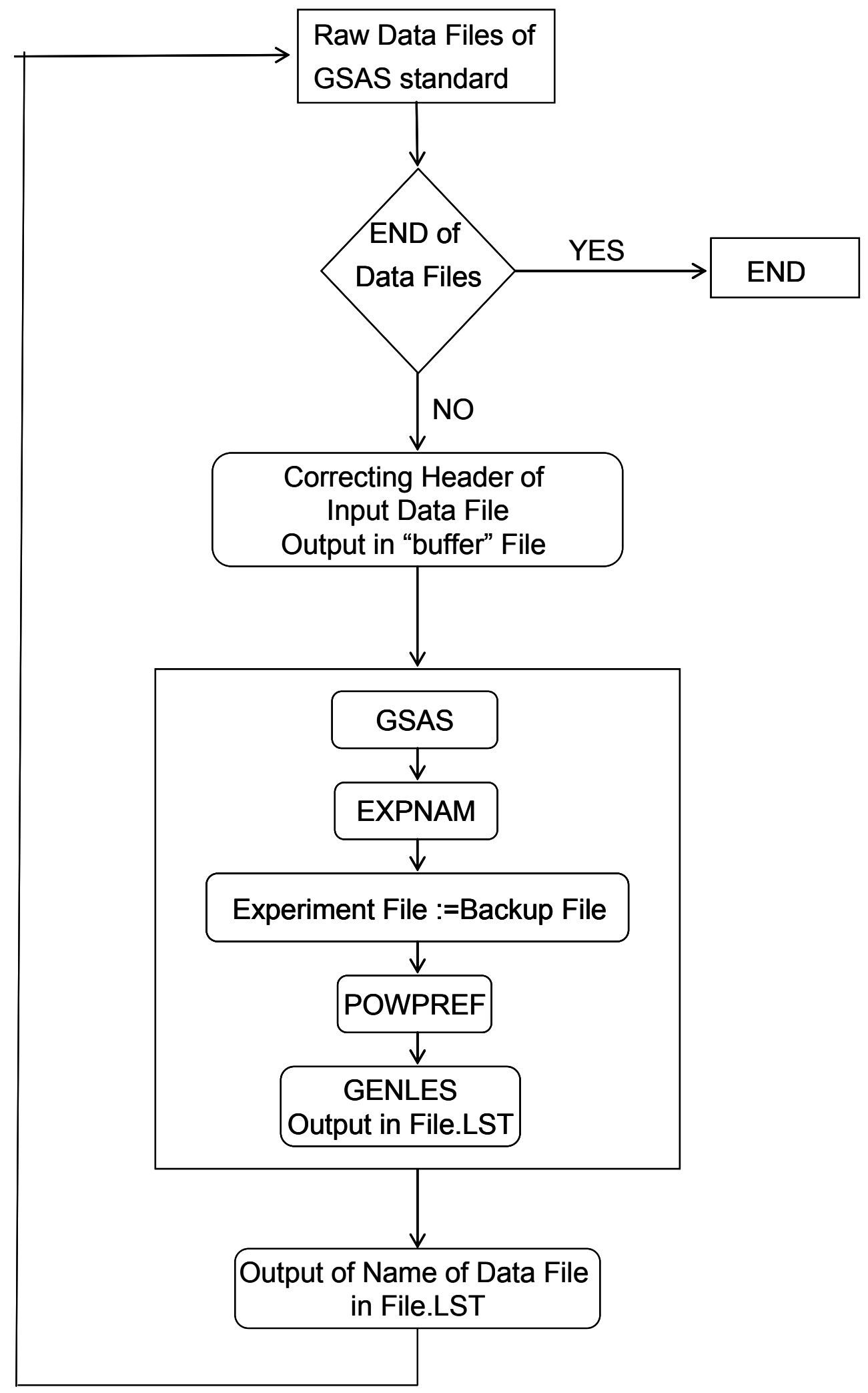

Figure 14. Algorithm of the automatic processing of the kinetic data 
The automatically obtained gas hydrate fraction was plotted versus time and showed the trend of the hydrate formation. An example of the measured kinetics of the methane hydrate growth in deuterated and hydrogenated systems is shown in Fig. 15.
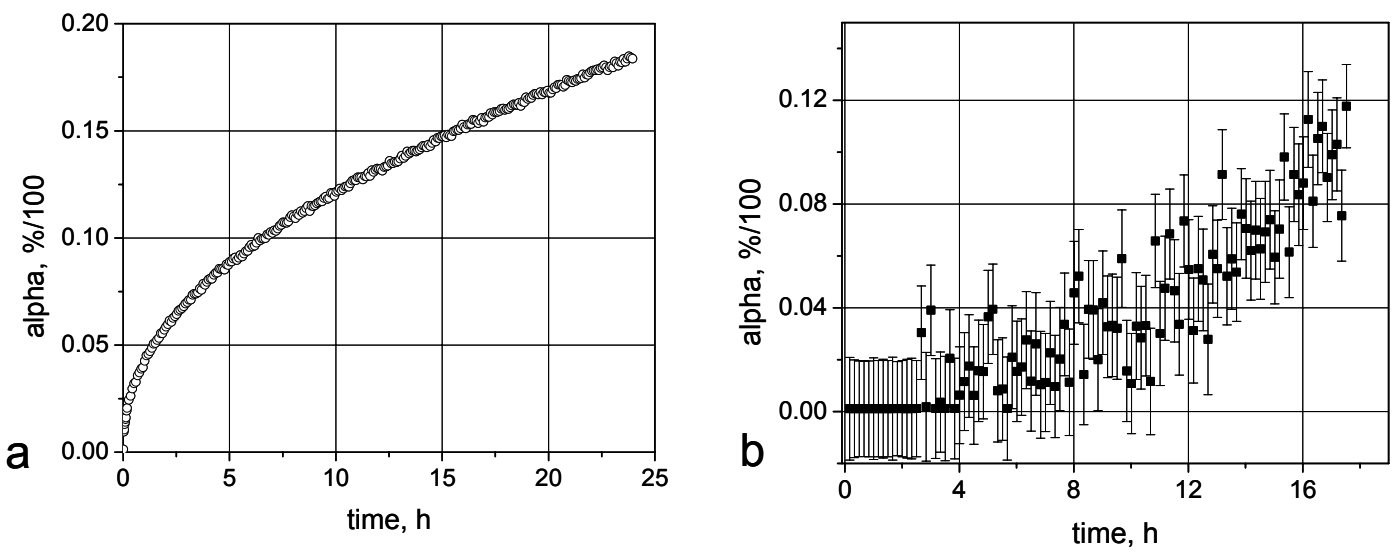

Figure 15. Plots of the weight fraction of methane clathrate (alpha) versus time obtained by the Rietveld refinement of the neutron diffraction data on the growth of (a) $\mathrm{CH}_{4}-\mathrm{D}_{2} \mathrm{O}$ at $6.0 \mathrm{MPa}$ and $263 \mathrm{~K}$ and (b) $\mathrm{CH}_{4}-$ $\mathrm{H}_{2} \mathrm{O}$ at 6.0 MPa and 264K. Each symbol shows a value of type-I weight fraction obtained from one data file. The estimated standard deviations of the refined values were in the limits of the plotted symbols (a) or denoted by error bars (b).

The relative amount of clathrate in the deuterated samples was obtained with eight times better precision than in the hydrogenated ones (Appendix A.3, A.5). The high incoherent scattering cross-section of the hydrogen (see Table 2, Ch. 3.1.5) and the small amount of $\mathrm{H}_{2} \mathrm{O}-\mathrm{CH}_{4}$ phase during its initial formation made it difficult to use the neutron diffraction method for in situ measurements of the gas hydrate growth in hydrogenated systems. Admittedly with a low precision, the relative amount of the forming hydrogenated clathrate was deduced in situ for the first time. 


\subsection{X-ray Diffraction Method}

High-energy synchrotron radiation registered by an area detector was the tool chosen to perform in situ time-resolved X-ray diffraction studies of the methane clathrate growth.

\subsubsection{Synchrotron Radiation and Diffraction Set-Up}

An acceleration of charged particles, guided by a magnetic field in a circular path, causes the emission of electromagnetic radiation, called synchrotron radiation. The acceleration of the particles is provided at certain points of their trajectory by a high frequency electric field. The wavelength and the intensity of the synchrotron radiation depend on the type of emitting particles and their energy. The obtained electromagnetic radiation of a very intense, highly collimated, polarized beam with an wavelength ranging from $10^{-2} \mathrm{~mm}$ to $10^{-2} \mathrm{~nm}$, is a convenient tool for studies of the condense matter. A coherent elastic scattering of X-ray photons, incident to a target, is detected as an X-ray diffraction pattern, which can give the atomic structure of the target.

The synchrotron radiation used in the diffraction experiments is produced in storage rings. They provide a long-term containment of particle beams in high-vacuum beam tubes having a roughly circular shape. Fig. 1 shows a storage ring of positrons DORIS III, Hamburg.

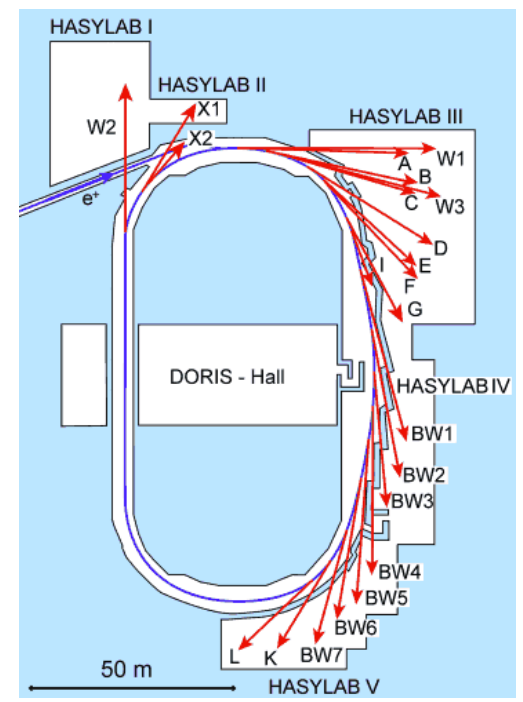

Figure 1. Rays-track geometry of DORIS III storage ring, DESY-HASYLAB, Hamburg. Injected particles are positrons with energy of $4.5 \mathrm{GeV}$. 
In situ studies of the gas hydrate kinetics required an equipment of high gas pressure and cryogenic devices (see Fig.2a). The set-up chosen for the X-ray measurements was similar to the one used for in situ neutron diffraction experiments (see Ch. 3.1.4). The thick-walled pressure cells (see Fig.2b), in which the samples were placed during the measurements, required a use of a hard X-ray diffraction technique.

Time-resolved X-ray diffraction data on the $\mathrm{CH}_{4}$-clathrate growth were collected at a beamline BW5 using photons emitted by an acceleration of positrons in the storage ring DORIS III, DESY-HASYLAB. During the beamline operation the intensity of the primary X-ray beam decreases as particles are lost from the ring and a periodical refilling with positrons is required.
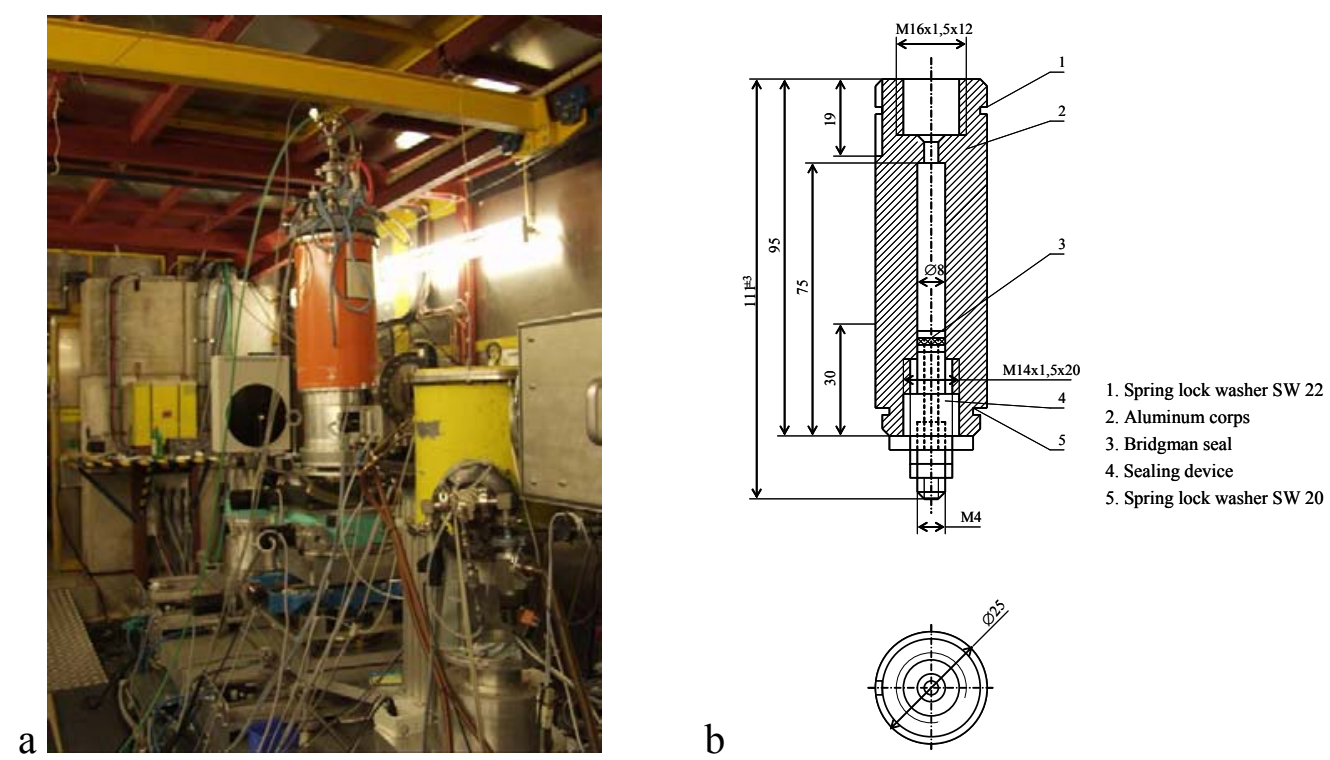

$\mathrm{b}$

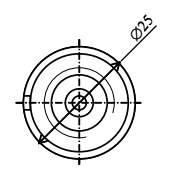

Figure 2. Standard "Orange cryostat" and high-pressure lines (a), and a sample placed in a pressure cell (b) were used for in situ $\mathrm{X}$-ray measurements of the methane clathrate kinetics.

The set-up made at the beamline BW5 to measure time-resolved diffraction data during methane clathrate growth is shown in Fig.3. A monochromized and collimated photon beam of about $100 \mathrm{keV}(\sim 0.124 \AA)$ was used as an incident X-ray beam that had a size of $1 \mathrm{~mm}^{2}$ at the sample position. An image plate detector, placed at 120-140 cm from the sample, was used to register the X-ray powder diffraction cones. One Bragg-angle absorber ring consisting of an Al$\mathrm{Pb}-\mathrm{Al}$ sandwich, positioned between the sample and the X-ray detector, absorbed the photons scattered by the Aluminium pressure cell at Bragg angles higher than $4-4.5^{\circ} 2 \theta$. 


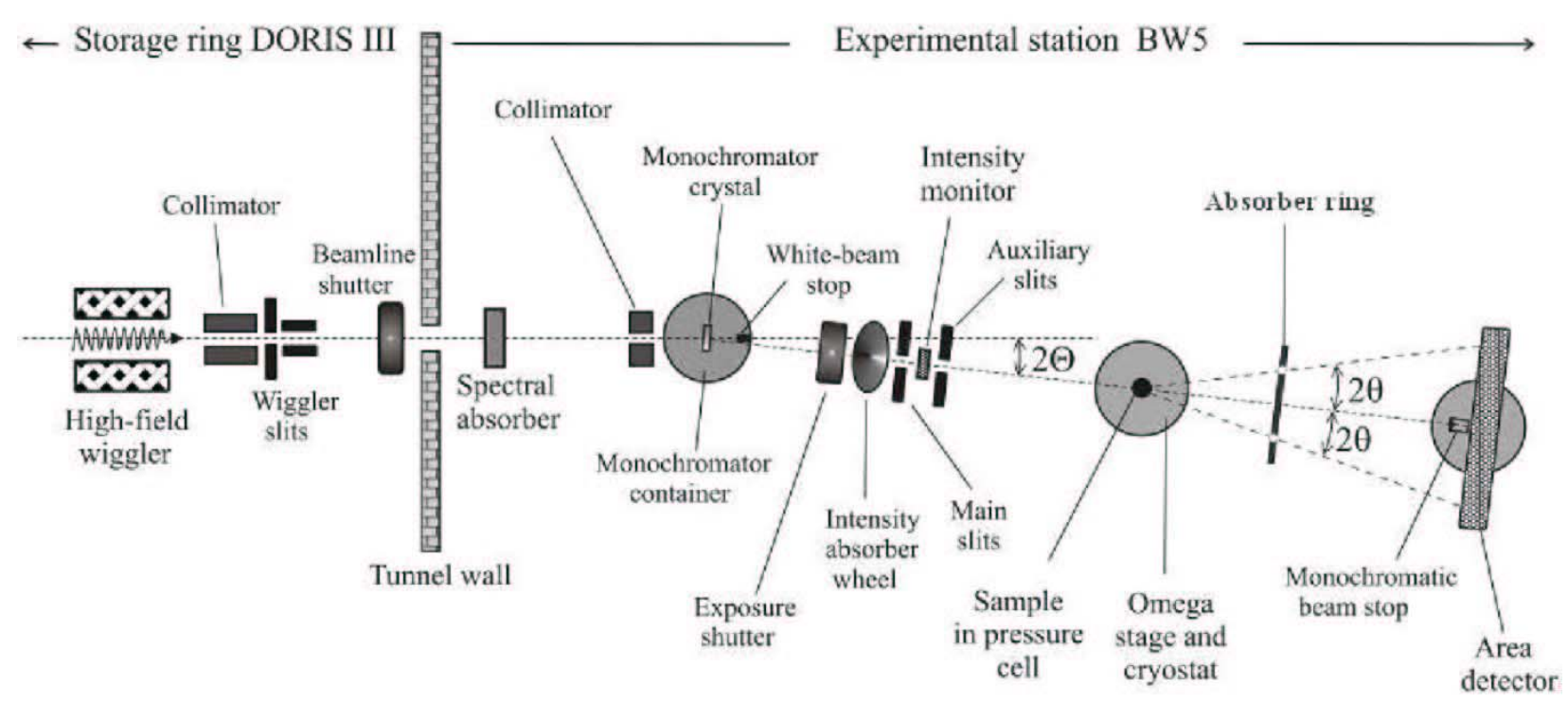

Figure 3. Schematic drawing of the set-up used for in situ $\mathrm{X}$-ray kinetic studies of the $\mathrm{CH}_{4}$-hydrate formation at BW5-HASYLAB, Hamburg.

The sample diffraction was then detected by an image plate detector MAR 345. Its major components are:

- a circular image plate with a diameter of $345 \mathrm{~mm}$ fixed to a circular platter mounted normally to the incident X-ray beam;

- a He-Ne laser/photomultiplier scanning head configuration which is moving radially along the active surface of the image plate;

- and an amplifier/ADC (Analog Digital Converter) unit, which converts the analogue photomultiplier signal into a digital one ready for storage on a hard disk and immediate use by an image display software.

The principle of work of an image plate detector is similar to the one of a photographic film. When the plate is exposed to X-rays an image is temporarily stored in a phosphor coating. After the exposure to the X-rays is completed the plate starts to rotate and a scanning unit moves from the edge to the centre of the plate reading the data in a spiral format. As the scanning head moves towards the center, the speed of the plate rotation increases to maintain a constant speed under the scanning head. When the scanning is completed the scanning head is withdrawn and strong lights are turned on to erase the plate. Erasing is necessary to remove any residual signal from the Xrays before the next exposure. The obtained digital image is then stored in a PC. 


\subsubsection{Long-Time Image Plate Detector Measurements - Problems and Outline}

The in situ X-ray diffraction was used to observe reactions of methane gas hydrate growth run in the same way as it was done for the in situ neutron diffraction studies (Ch. 3.1.6). The starting $\mathrm{H}_{2} \mathrm{O}$ - and $\mathrm{D}_{2} \mathrm{O}$-ice powder made by spraying technique (Ch. 3.1.5) was transported at a temperature of liquid nitrogen to BW5 at DESY-HASYLAB, Hamburg. Like in the neutron diffraction experiments (Ch. 3.1), each sample was placed in a cooled pressure cell at an applied small stream of gas and then the Bridgman seal was closed. After the sample was inserted in the cryostat and the temperature was equilibrated at a chosen value high gas pressure was applied and a data collection was started. Two-dimensional X-ray powder diffraction data were registered by an image plate detector. One plate diameter of 1800 pixels (or a diameter of $180 \mathrm{~mm}$ with a pixel size of $100 \mu \mathrm{m}$ ) was chosen for the data collection. The scanning and erasing of the image plate took 55s. Time resolved image data were taken during $\mathrm{CH}_{4}$-clathrate runs (see Table 1).

\begin{tabular}{|c|c|c|c|c|c|c|c|}
\hline \multirow{2}{*}{$\begin{array}{c}\text { Series } \\
\text { No. }\end{array}$} & \multicolumn{7}{|c|}{ Conditions of experiments } \\
\cline { 2 - 8 } & $\begin{array}{c}r_{i 0}, \\
\mu \mathrm{m}\end{array}$ & Ice Ih & $\begin{array}{c}T, \\
\mathrm{~K}\end{array}$ & $\begin{array}{c}P(f), \\
\mathrm{MPa}\end{array}$ & $\begin{array}{c}P_{d}\left(f_{d}\right), \\
\mathrm{MPa}\end{array}$ & $\begin{array}{c}\text { Excess } \\
\text { fugacity, } \\
\left(f-f_{d}\right) / f_{d}\end{array}$ & $\begin{array}{c}\text { Duration, } \\
\mathrm{h}\end{array}$ \\
\hline \multirow{3}{*}{1} & \multirow{3}{*}{35} & $\mathrm{D}_{2} \mathrm{O}$ & 272 & $6.00(5.20)$ & $2.5(2.36)$ & 1.2 & 4 \\
\cline { 3 - 8 } & & $\mathrm{H}_{2} \mathrm{O}$ & 268 & $6.00(5.16)$ & $2.18(2.07)$ & 1.49 & 10.5 \\
\cline { 3 - 8 } & & $\mathrm{H}_{2} \mathrm{O}$ & 258 & $6.0(5.05)$ & $15.7(15)$ & 2.37 & 14 \\
\hline \multirow{3}{*}{2} & \multirow{3}{*}{3} & $\mathrm{D}_{2} \mathrm{O}$ & 268 & $6.00(5.16)$ & $2.18(2.07)$ & 1.49 & $55^{*}$ \\
\cline { 3 - 8 } & \multirow{2}{*}{27} & $\mathrm{D}_{2} \mathrm{O}$ & 253 & $6.0(4.99)$ & $13.4(12.9)$ & 2.87 & 24 \\
\hline & & $\mathrm{D}_{2} \mathrm{O}$ & 268 & $6.00(5.16)$ & $2.18(2.07)$ & 1.49 & 25 \\
\cline { 3 - 8 } & $\mathrm{H}_{2} \mathrm{O}$ & 263 & $6.0(5.11)$ & $18.4(17.5)$ & 1.92 & $80^{*}$ \\
\cline { 3 - 8 } & $\mathrm{H}_{2} \mathrm{O}$ & 253 & $6.0(4.99)$ & $13.4(12.9)$ & 2.87 & 26 \\
\hline
\end{tabular}

* full period of intermittent runs

Table 1. Experimental p-T conditions of $\mathrm{CH}_{4}$ gas hydrate formations studied in situ by $\mathrm{X}$-ray diffraction method.

Each image was a result of a collection of the diffracted photons in the detector during a horizontal rotation of the cryostat at $\omega$ of 4 degree/min. The rotation of the sample during its 
imaging was done to obtain smooth Debye-Scherrer rings instead of spotty ones corresponding to an appearance of single grains at a fixed sample position. Additionally the sample rotation increased the measurement statistics and improved the peak-to-background ratio and the peak shape. Prior to starting a data acquisition, single snapshots of 1-5 min were taken to check whether the Debye-Scherrer rings were well visible. Thus, the time of data acquisition was chosen basically by eye. The time period/per image used for the first set of runs was initially 1 and $5 \mathrm{~min}$ for the fast reaction part and $20 \mathrm{~min}$ for the slower part. For the second and third measurement campaign a constant acquisition time of 5 and 3 min was used, respectively. The image data processing software FIT2D of Hammersley (2003) allowed an integration of the intensities of the Debye-Scherrer rings of each image giving a one-dimensional diffraction pattern (see Fig. 4). 

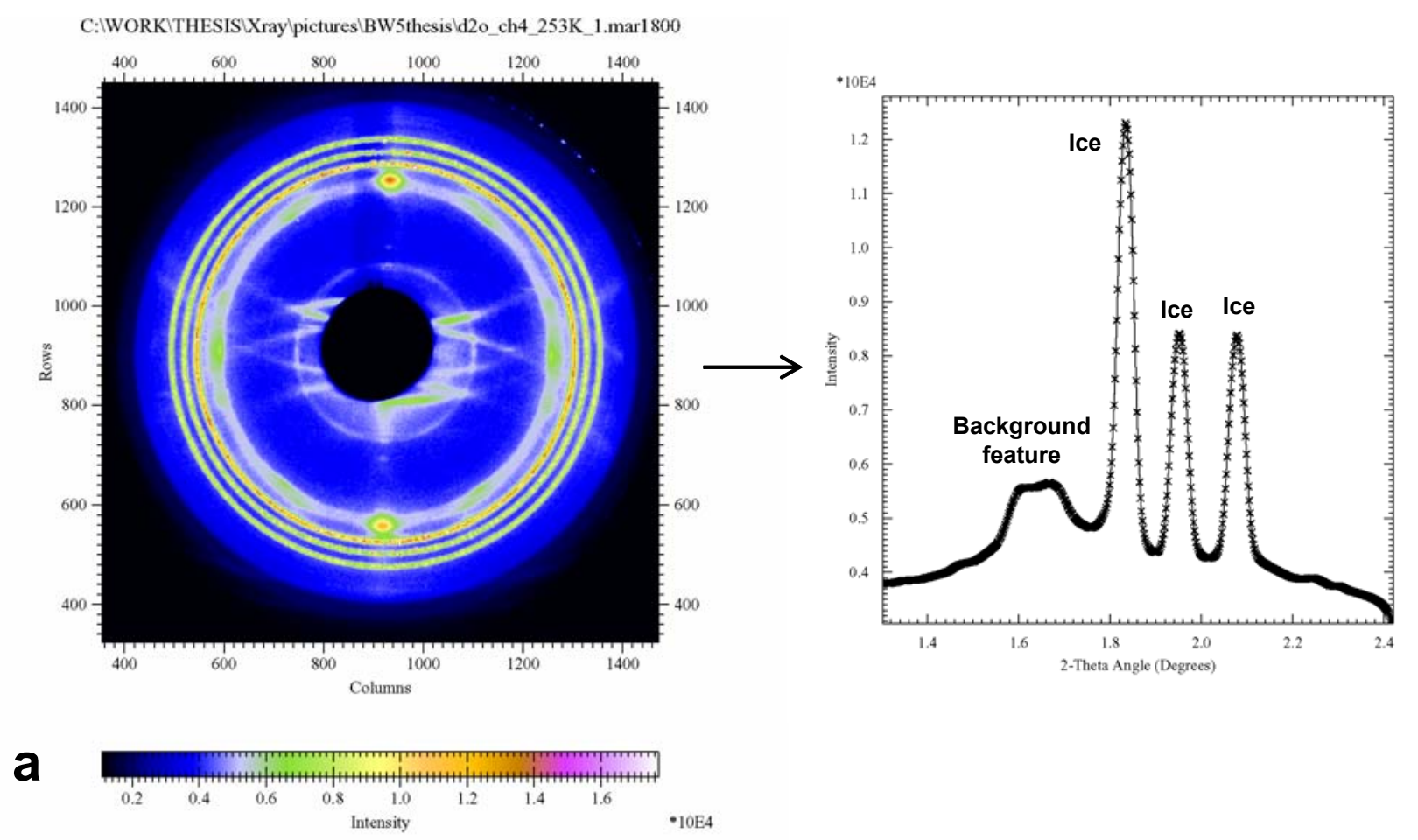

C: $\mid$ WORK $\backslash T H E S I S \backslash X r a y \backslash$ pictures $\backslash B W 5$ thesis $\backslash$ 20_ch4_253K_120.mar 1800
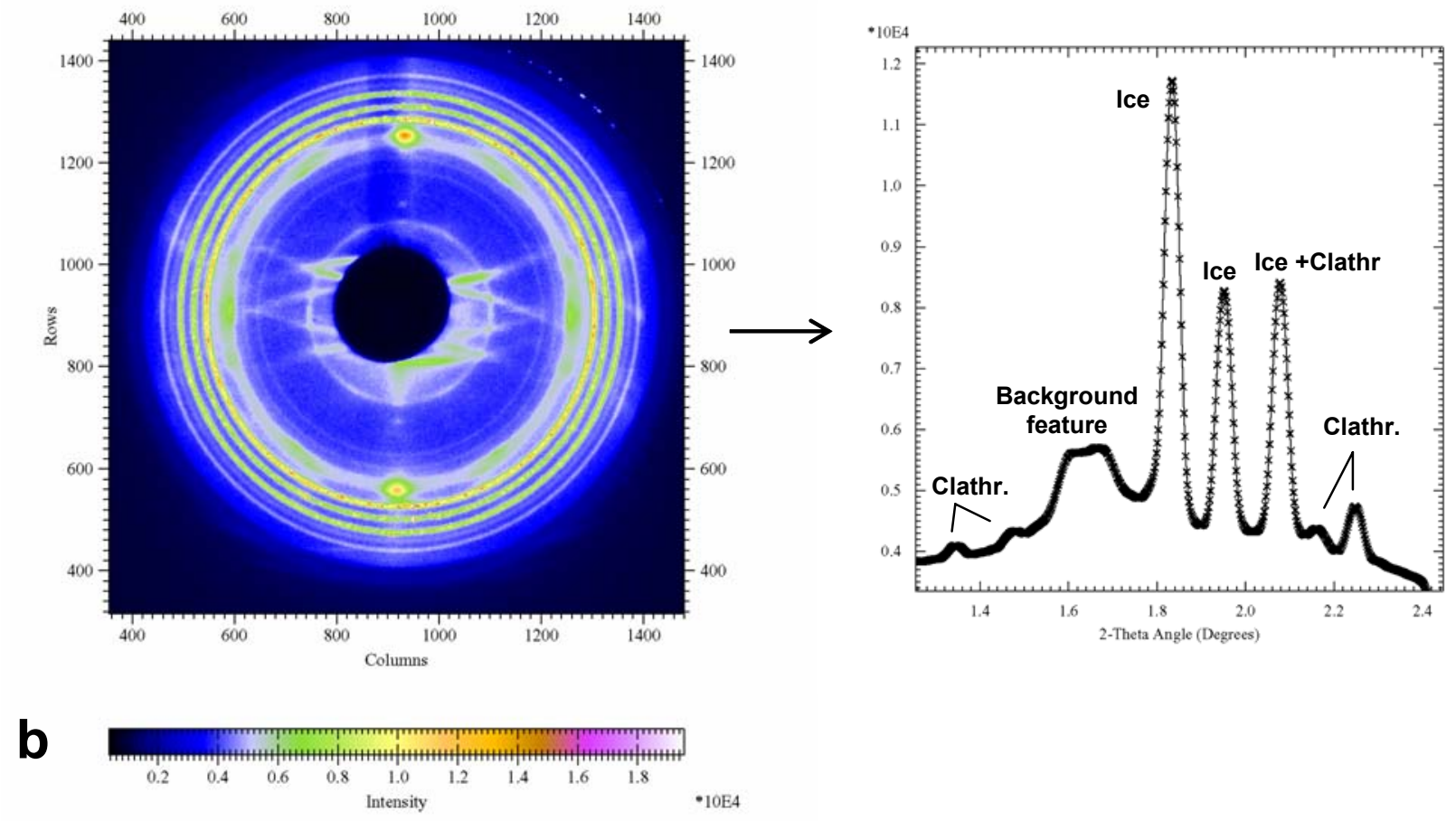

Figure 4. X-ray diffraction patterns of $\mathrm{D}_{2} \mathrm{O}-\mathrm{CH}_{4}$ gas hydrate forming at $6.0 \mathrm{MPa}$ and $-20^{\circ} \mathrm{C}$ after 6 min (a) and $24 \mathrm{~h} \mathrm{(b)}$ of reaction were registered by an area detector as Debye-Scherrer rings. The integration of the intensities along the rings resulted in one-dimensional diffraction patterns (pictures on right). The background feature originates from precipitates of the pressure cell. 
It was done as follows: geometrical corrections of pixel intensities were applied by a refinement of the beam center and any non-orthogonality of the detector to the main beam. Then intensities of pixels at equal 2-theta from the beam center (intensities of Debye-Scherrer rings) were integrated and normalized to the number of contributing pixels.

Each obtained output 2-theta diffraction pattern in a GSAS format was processed by a Rietveld refinement method (see Ch. 3.1.2). The automatic procedure developed for the processing of the neutron diffraction data (see Ch. 3.1.8) was applied for the X-ray kinetic data as well. As a result the ice-to-hydrate ratio was determined for each data set and the growth of the phase fraction of methane hydrate with time was plotted. The growth of $\mathrm{D}_{2} \mathrm{O}-\mathrm{CH}_{4}$ clathrate at $20^{\circ} \mathrm{C}$ and 6.0 MPa for a period of $24 \mathrm{~h}$ is shown as a typical example of the obtained kinetic results (see Fig. 5a). The gas hydrate structure model of Klapproth (2002) was used for Rietveld refinement of the data (see Appendix B). The observed variations of the clathrate phase fraction were unphysical and were unlikely to come from the measured sample. A correlation of these variations with intensity changes of the incident X-ray beam, measured by a monitor (a $\mathrm{Si}$ semiconductor detector) was observed (Fig. 5b). A slow increase of the hydrate fraction (respectively a decrease of the ice fraction) during an intensity drop of the primary photon beam caused by a decrease of the positron current in the storage ring, was observed. When the ring was refilled with positrons the primary photon beam jumped and a decrease in the hydrate fraction was obtained. In order to avoid a possible influence of the intensity change on the results of the phase fractions in the sample, the one-dimensional diffraction data were corrected for the variable incident X-ray beam. The intensities $I_{\exp }^{i}$ recorded in each data file $i$ were normalized using the intensity of the related monochromatic beam $I_{M}^{i}$. A normalization factor $N_{i}=\frac{I_{M}^{i}}{I_{m}^{\min }}$, where $I_{m}^{\min }$ is a minimum of the counts registered by the beam monitor over the reaction period, was calculated. Then the diffraction data $i, i=1 . . n$ with normalized intensities $I_{N}^{i}=\frac{I_{\text {exp }}^{i}}{N_{i}}$ were processed. The obtained kinetic results were not much improved (see Fig. 5c). Other normalization factors using the current of the storage ring or integrals over specific parts of the diffraction pattern (background ranges) did not change the result of clathrate formation shown with Fig.5c. 

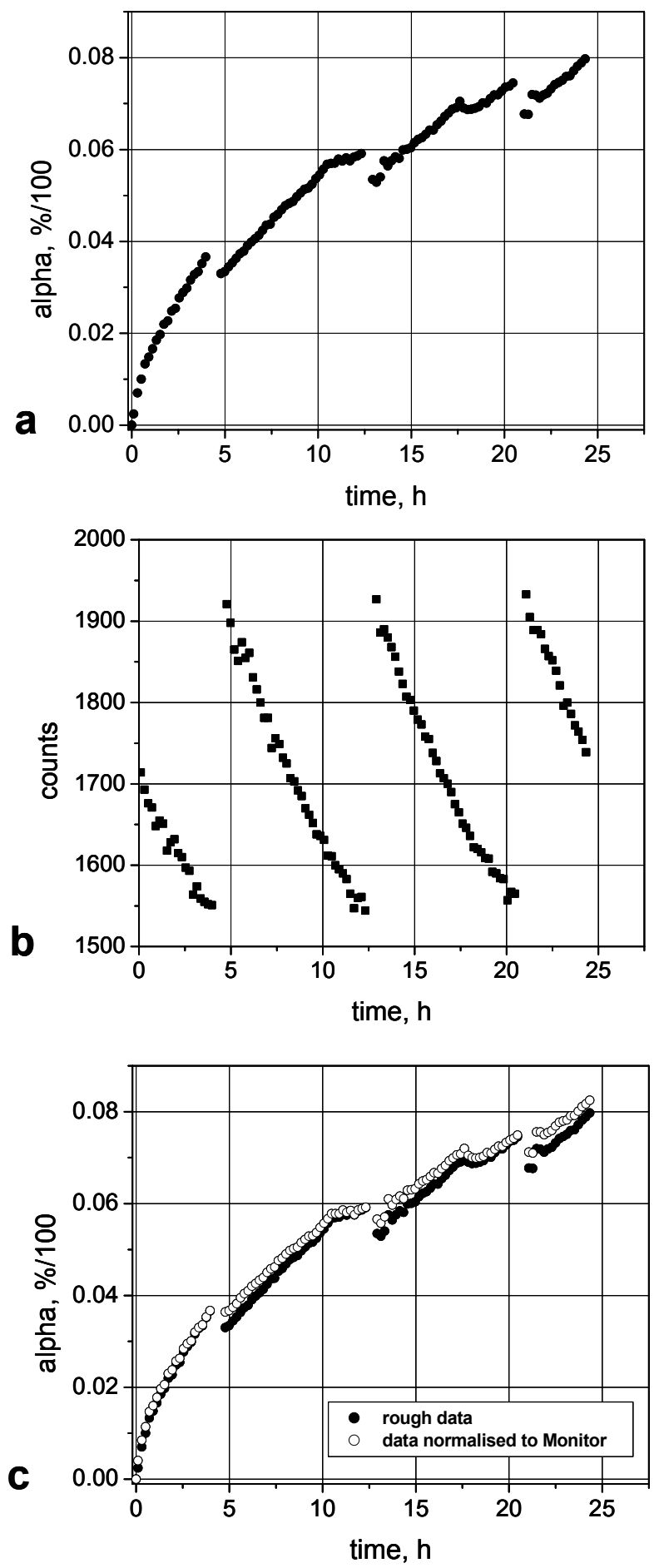

Figure 5. Growth of $\mathrm{D}_{2} \mathrm{O}-\mathrm{CH}_{4}$ hydrate at $-20{ }^{\circ} \mathrm{C}$ and $6.0 \mathrm{MPa}$ (a) obtained by GSAS. Monitor counts registered during the reaction (b) were used to normalize the measured intensities but the obtained results of the gas hydrate fraction vs time were still unsatisfying (c) (see text). 
Then the source of this problem was searched in the image plate used to collect the twodimensional diffraction data. The starting ice material was not a good powder and it was found that sometimes single ice grains or clusters of grains could cause an overload of the detector at some pixels as it is shown for example with Fig.6. According to the characteristics of the detector, the response of the detector for X-rays is linear within its dynamic range. The dynamic range of mar 345 image plate detector is 0 to 131000 counts per pixel, limited by the 17 bits used by the ADC unit. A value of 65535 counts (or a 16-bits integer number) is the maximum intensity per pixel, which is allowed for a mar345 format. When a larger amount of photons reaches the detector, an overflow in the data record occurs and a float value of 9.999990E5, instead of the integer one, is assigned to the related image pixel. This was the reason the integrated intensity of the strongest ice reflection of (100) to have an irregular shape (Fig.6a-b). An applied mask threshold of 1E5 counts per pixel made the pixels with float values to be skipped during the intensity integration. As a result the shape of (100) reflection was smoothed (Fig.6c). It should be mentioned that the detector was overloaded only at some pixels corresponding to that particular Bragg peak. 

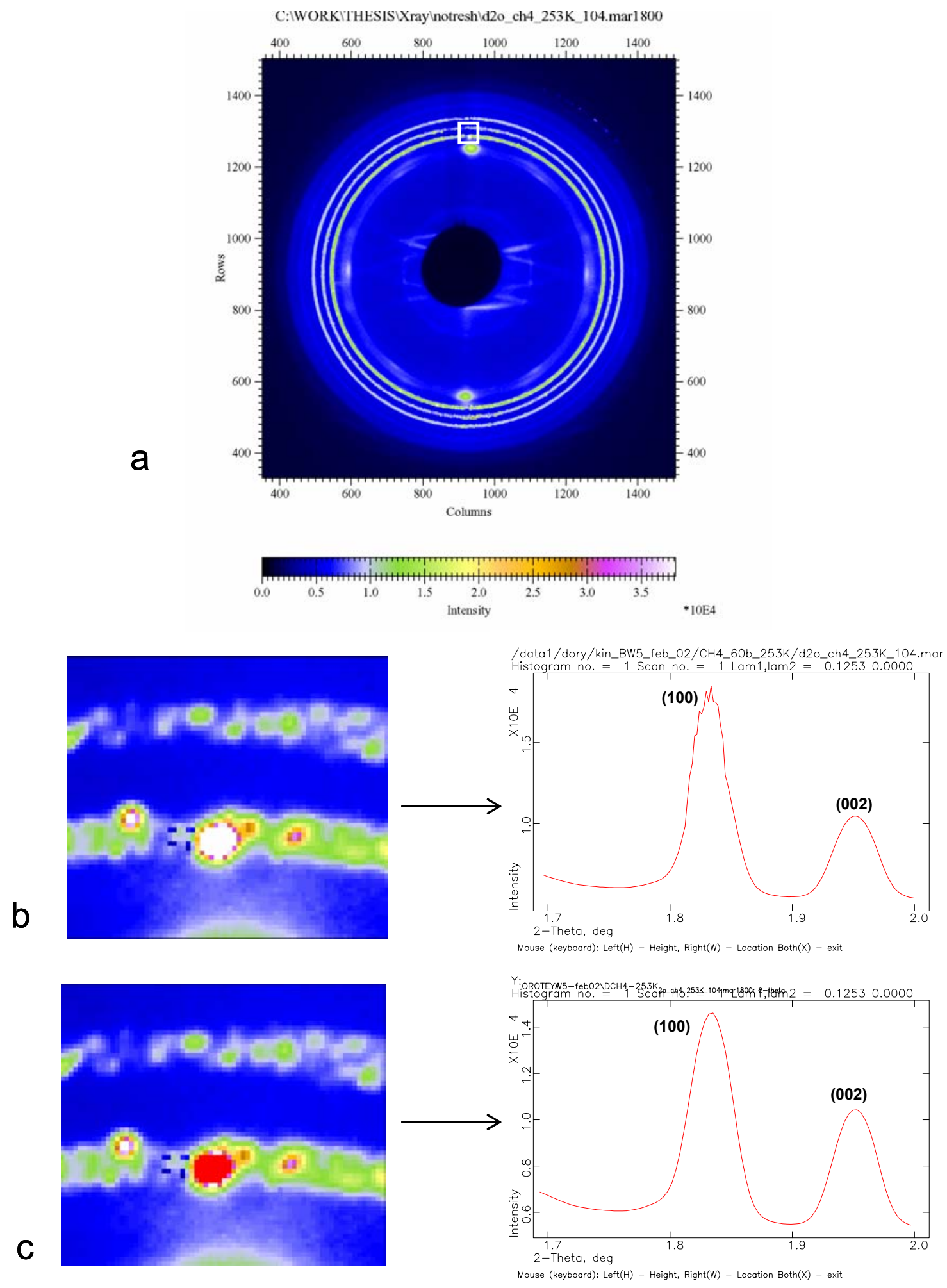

Figure 6. FIT2D plot (a) of $\mathrm{D}_{2} \mathrm{O}-\mathrm{CH}_{4}$ hydrate formed after $21 \mathrm{~h}$ of reaction at $-20^{\circ} \mathrm{C}$ and $6.0 \mathrm{MPa}$. A large ice grain (small squire) can cause overloading (b) of some pixels of the detector (white area) giving an irregular shape of the integrated Bragg intensity of (100) reflection. A threshold mask applied to the overloaded pixels (c) improved the integrated Bragg peak intensity. 
An automatic integration of two-dimensional data $\left(\mathrm{D}_{2} \mathrm{O}-\mathrm{CH}_{4}\right.$ growth at $\left.6.0 \mathrm{MPa},-20^{\circ} \mathrm{C}\right)$ at an applied threshold of 1E5 was performed by a macro made in FIT2D. The obtained 2-theta diffraction patterns of GSAS format were again analysed by the Rietveld refinement and the results were compared with the previous ones (see Fig.7).

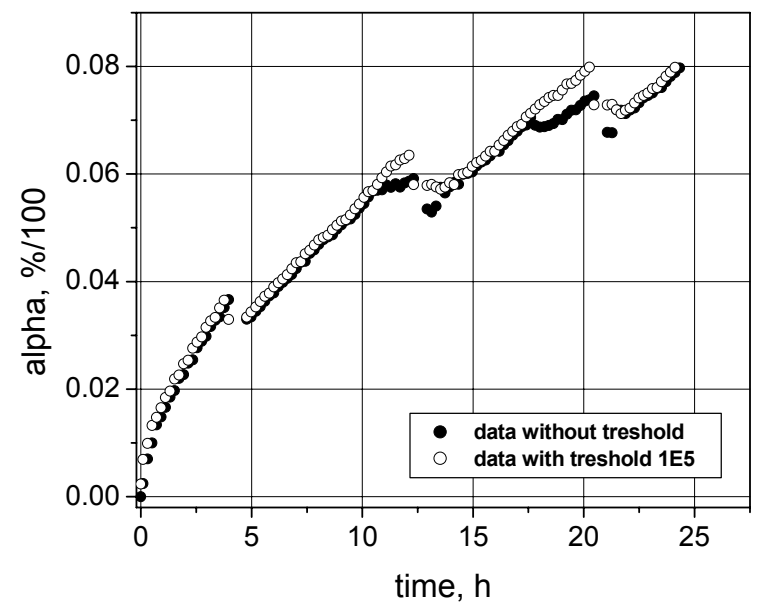

Figure 7. Comparison of the methane clathrate fraction deduced by the Rietveld refinement of GSAS data obtained from two-dimensional data with and without applied threshold of 1E5 counts/pixel.

A smoothing of the kinetic curve was observed at the points of overloading of the detector. Nevertheless the general trend of the methane clathrate kinetics, showing unexplained jumps and variable slopes with time, was not much improved.

In order to find out the source of the problem, described above, a rough check of the intensities of the one-dimensional diffraction patterns was done. The strongest intensities of two ice and one clathrate Bragg reflections with Miller indices of (100), (002) and (321), respectively were deduced for about 50 data files manually. A smooth change in the ratio of the maximum intensities $\frac{I_{002}}{I_{321}}$ of an ice and a clathrate reflection was observed (see Fig.8a). It behaved as independent on the jumps in the intensity of the primary beam. Such a result is logical because of the linearity of the detector, i.e. the image plate would register less intensity at less incident photons and thus, the ratio of the intensities of the Bragg peaks should not be affected. 

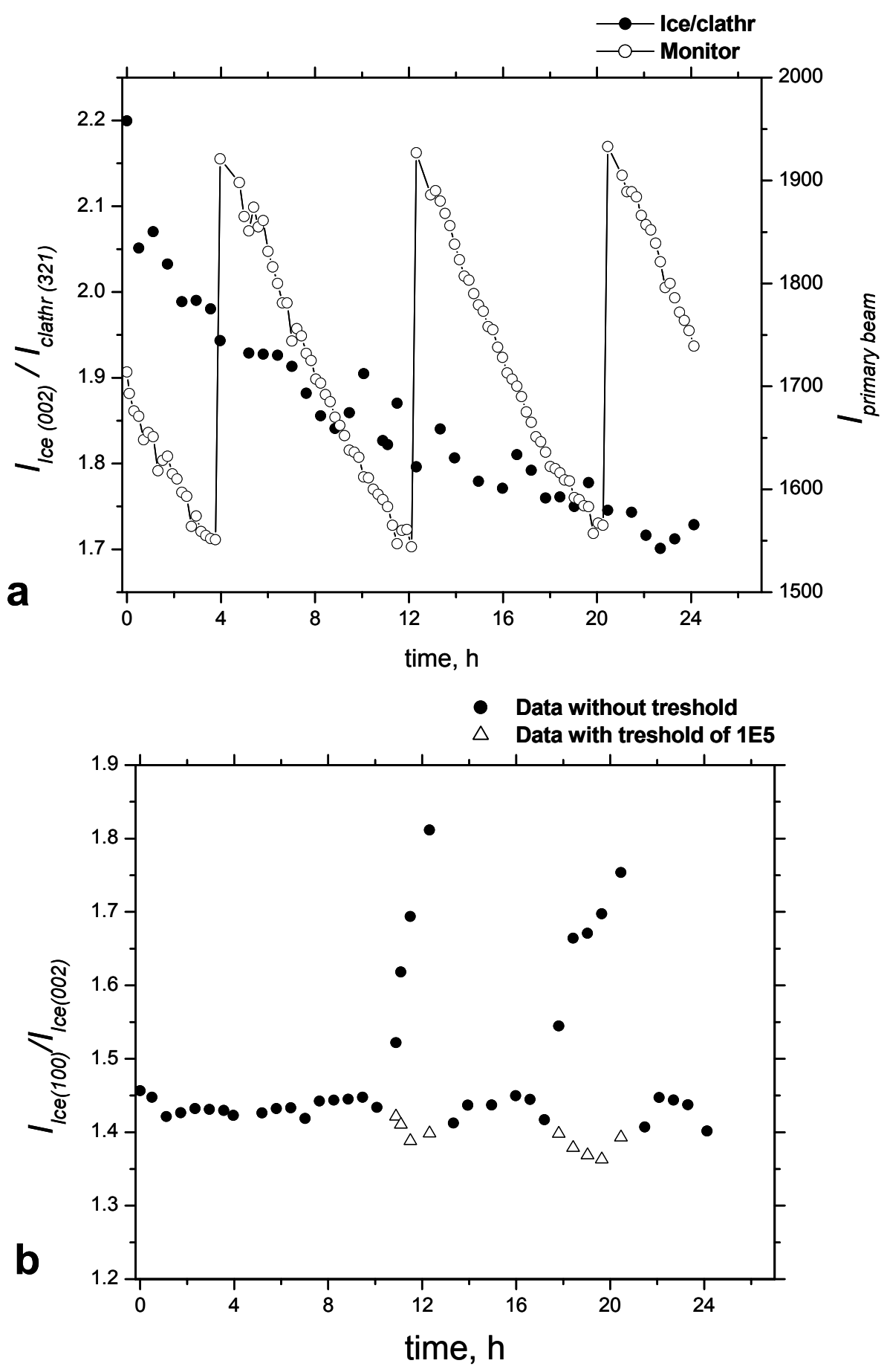

Figure 8. Plot of the ratios of the maximum intensities of (002) ice- and (321) clathrate reflections (a) calculated from the rough data (black circles) showed a smooth change with the primary beam (white circles). The overloading of detector pixels, observed only at the ice reflection of (100), caused erratic changes in the ratios of (100) and (002) intensities (b) that can be corrected with an intensity threshold (white triangles). 
As it is seen on the plot (Fig. 8b) the ratio of the maximum intensities of two ice reflections showed jumps caused by the appearance of large ice grains at (100) crystallographic plane. A threshold of pixel intensities used during the intensity integration smoothed the variation of the calculated ratio. Such a small variation may affect the fraction results but could not explain the observed the erratic jumps and changes of the kinetic slopes.

Then the main problem was found in the automatic data processing. The correlations of the different parameters of the calculated diffraction pattern (e.g. Eq. 3-5, Ch. 3.1.2) have to be considered when the Rietveld refinement method is applied. The parameters of the histogram scale and the clathrate and ice phase fractions correlate between each other and could not be refined simultaneously. Thus, the values of two of them, for example the histogram scale and the phase fraction of clathrate, were refined when the third one was fixed (e.g. phase fraction of ice). The only a way found to describe the background of the X-ray data in GSAS was by using manually fixed background values plus cosine Fourier series with only one parameter (independent on 2 $\theta$ ). There was a problem that if the calculated background does not match the measured one, the refined value of the histogram scale would be affected because of the parameters' correlation. The refined parameters then were the phase fraction of clathrate, the histogram scale and one background parameter. When all the data were automatically refined by GSAS the correlations described above affected the calculation of the ice-to-hydrate ratios.

One possible solution is the creation of a new program, which locates in each file the background and peak intensities within a certain criteria. Then an automatic run of this program with all data files has to be developed. To make such a tool was not possible in the remaining time of the $\mathrm{PhD}$ work. 


\subsection{Gas-Consumption (pVT) Method}

As it was shown before (Ch. 3.1) the neutron diffraction is the best tool to follow in situ the gas hydrate formation kinetics, in particular for the very initial part of the reaction. The limited beam time allocation on the neutron sources required a search of an alternative method for in situ studies of the hydrate kinetics. The use of a laboratory technique based on gas consumption during the formation reaction (pVT method) was found as a solution for following in situ the hydrate reaction.

\subsubsection{Method Basics}

Gas and ice molecules are the building units of a newly forming gas hydrate structure. Therefore, if at least one of the initial phases has a limited number of molecules, a measure of their decrease caused by a transition to a new phase would correlate with the rate of growth. Gas hydrate formation can be observed in situ either by a neutron diffraction record of ice consumption (Ch. 3.1) or by a continuous pressure record during the reaction. The latter is the so called gas consumption (pVT) method, which uses the pressure drop during the gas hydrate growth in a closed ice-gas system to measure the reaction rate, i.e. the amount of clathrate formed per unit time. The ideal gas law (Eq. 18, Ch.2) gives the relation $P=\frac{N}{V} k_{B} T$ between the pressure at a given temperature and the number of gas molecules $N$ per unit volume $V$ (gas density). A change of the gas density would cause a change in the measured pressure. If there is no supply of gas during the gas hydrate formation the pressure drops because of the encaging of gas molecules in the forming clathrate cages. The rate of measured pressure drop correlates with the rate of trapping of gas molecules in the growing gas hydrate structure and it is proportional to the reaction rate.

\subsubsection{Set-Up and Data Collection}

The set-up used for the in situ laboratory reaction runs was the same as the one for the neutron diffraction experiments (see Ch. 3.1.4) except that the low temperature in the gas-consumption measurements was provided by a low temperature bath instead of a He-flow cryostat (Fig. 1). 


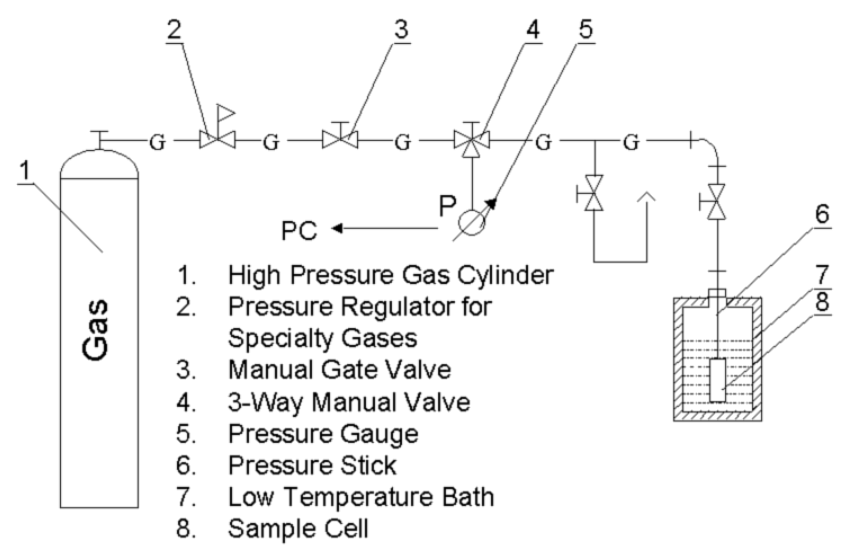

Figure 1. High pressure/low temperature set-up used for in situ measurement of the gas consumption during the methane gas hydrate growth.

The used bath was a type of Neslab RTE 140 Bath/Circulator with Microprocessor Controller. The bath temperature, provided by a circulation of a fluid, is controlled with accuracy of $\pm 0.1^{\circ} \mathrm{C}$. Temperature range is from $-40^{\circ} \mathrm{C}$ to $150^{\circ} \mathrm{C}$.

During the laboratory runs pressure was measured by a piezoelectric pressure sensor (No 5, Fig.1) of model ASHCROFT Type KXD, which was calibrated regularly with Heise mechanical manometers. According to the technical manual the pressure sensor works within a range of $0-400$ bar with an accuracy of $0.25 \%$ and it is linear within the temperature interval of $0-50^{\circ} \mathrm{C}$. Variations in the ambient temperature within this interval can give a measurement uncertainty of $\pm 0.3 \% / 10 \mathrm{~K}$ at a reference temperature of $20^{\circ} \mathrm{C}$ (zero point). An RS232 serial communication between the sensor electronics and the PC was established and permitted a continuous data acquisition of the gas pressure. A commercial 32-bit software Windmill 5 with COMIML - serial device module for RS232 - was used.

Two set-ups, as the one shown in Fig. 1, were used to study the methane clathrate kinetics. Both systems were connected to a common gas source and used calibrated pressure sensors to measure the pressures during the reactions. The pVT systems, called Stick 1 and Stick 2, are shown in Fig.2. 


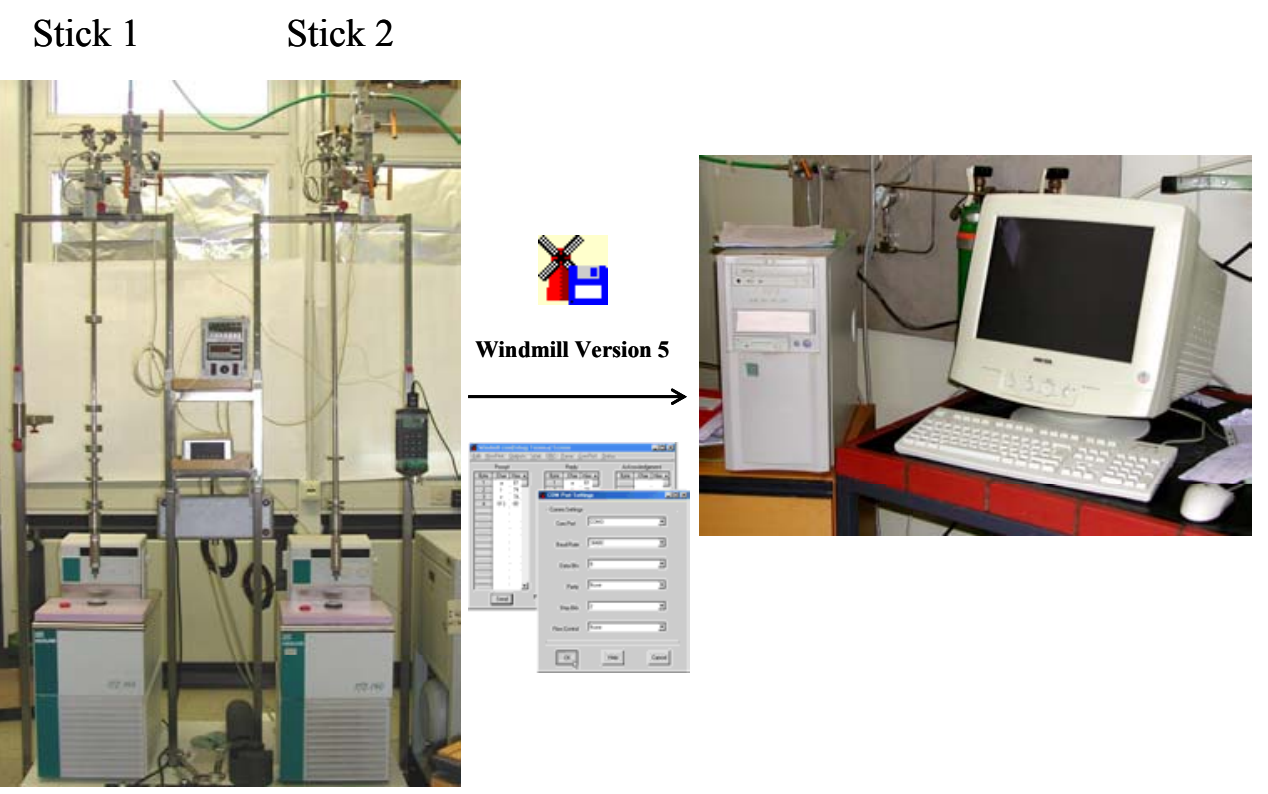

Figure 2. Pressure sticks and temperature baths used for in situ gas-consumption measurements at the laboratory (Göttingen). Values of the gas pressure during the reactions were recorded in a PC.

A measurement of an absolute value of the free volume of each set-up was difficult to perform with the desired precision. For the sake of comparison it was sufficient to determine the volume ratio of the both set-ups. It was done as follows: each system was closed at ambient pressure by the 3-way manual valve (Fig.1). Then the pressure lines were filled with methane gas up to the closed valve and pressure of 6.0 $\mathrm{MPa}$ was applied. After the opening the valve of each stick, a pressure drop $P_{i}, i=1,2$, depending on the volume of the stick, was obtained. The ratio of the volumes $\frac{V_{\text {Stick } 2}}{V_{\text {Stick } 1}}=\frac{P_{1}}{P_{2}}$ (law of Boyle-Mariotte) was measured at three different temperatures (Fig. 3) and a weak temperature dependence within a wide temperature interval of about $30{ }^{\circ} \mathrm{C}$ was observed. 


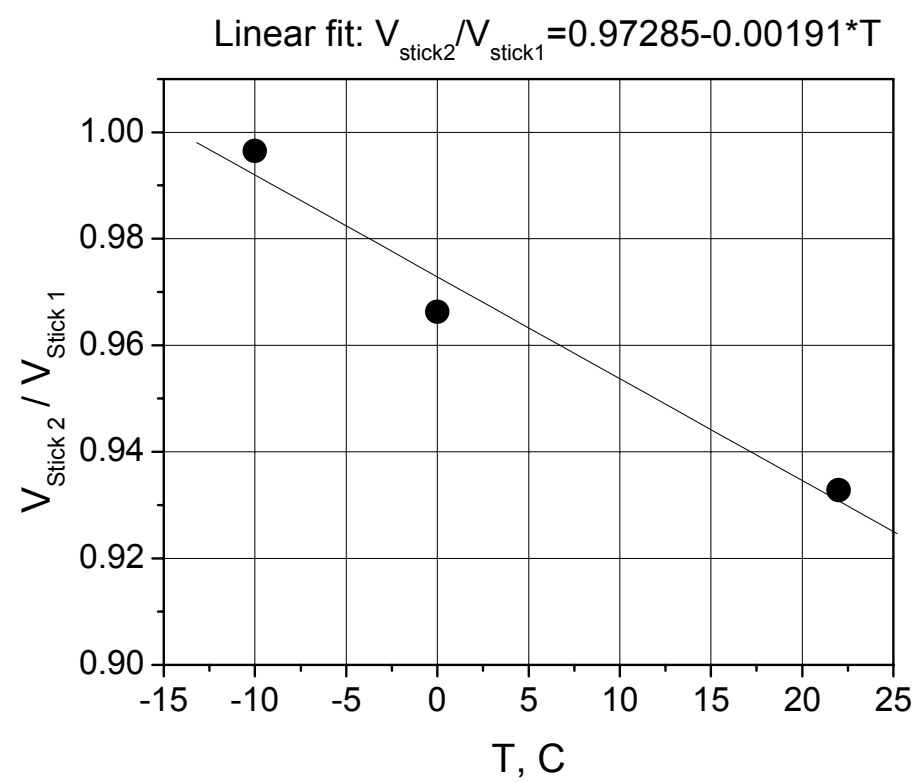

Figure 3. Volume ratio of the two set-ups (Stick 1 and Stick 2) of the gas-consumption measurements at the laboratory (Göttingen).

The gas-consumption method relies on the pressure drops to obtain the kinetics of gas hydrate growth. In order to prevent any income or outcome of gas in the reacted system it had to be completely closed. Thus, precise checks of both set-ups for possible leaks through the connections between the high-pressure units were made before each reaction run. Prior to starting a measurement the pressure stick was closed at applied high pressure and watched for a pressure drop for few hours. If a leak was not observed the system was considered as closed.

An ice powder with a typical grain size of $54 \mu \mathrm{m}$, prepared and stored in the way described previously (see Ch. 3.1.5), was used as a starting material to perform the pVT kinetic runs. Before starting of the reactions the pressure cell was cooled in liquid nitrogen for few minutes. The high-pressure lines were flashed by methane gas. An opening of the manual valve (No 4, Fig.1) provided a small stream of gas when the ice sample was inserted into the pressure cell, fixed to the sample stick. Then the Bridgman seal and the manual valve were closed. The pressure cell was inserted in the bath, already adjusted at a chosen temperature, and left for about 10 minutes to equilibrate. Then by an opening of the manual valve desired gas pressure was applied and the valve was closed. At the same time, the data acquisition of the pressure in the closed ice-gas system was started. The reaction of gas hydrate formation was observed by the pressure drop with time. The time steps of the data collection were $30 \mathrm{~s}$ or $1 \mathrm{~min}$ for the initial 
fast part of the reaction and 5, 10 or 15 min for the later slower parts of the reaction. The pressure in the system was adjusted manually from time to time in order to maintain it within typically a few percent of the desired value. The recorded pressure drop was then converted to an amount of gas consumed with time. An example kinetic run of $\mathrm{CH}_{4}$-clathrate formation observed in situ by the gas- consumption (pVT) method is shown on Fig.4.

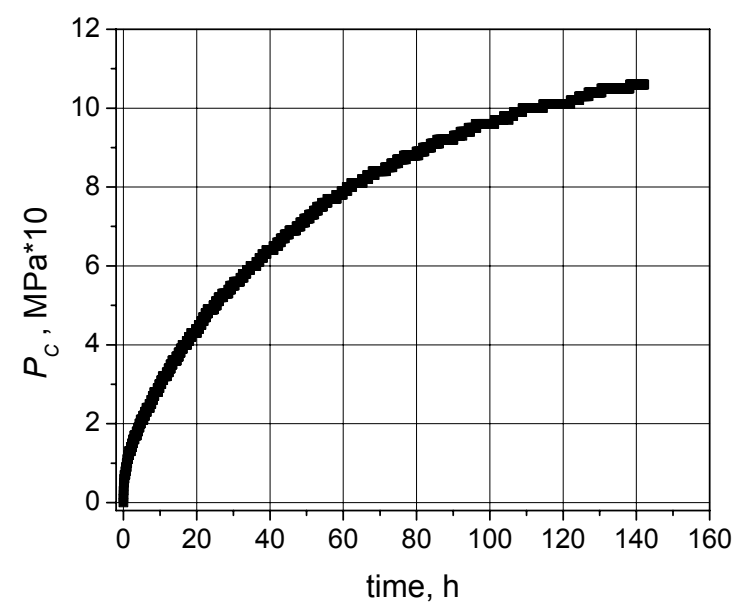

Figure 4. Gas consumption $P_{C}$ during a formation of $\mathrm{CH}_{4}-\mathrm{H}_{2} \mathrm{O}$ gas hydrate at $-10^{\circ} \mathrm{C}$ and $6.0 \mathrm{MPa}$.

It was observed that the pressure records of the summer reactions varied much more than the winter ones. Then the ambient temperature was measured by contacted to the pressure gauges temperature sensors of type EXTECH 421508 with thermocouple K type with accuracy of $\pm\left(0.05 \%+0.3^{\circ} \mathrm{C}\right)$. At applied high pressure it was found that by a holding the stick capillary by hand for about 5 min the pressure was increased with 0.3 bar for $5{ }^{\circ} \mathrm{C}$ increase of the measured temperature. The similar effect was observed when the pressure sensor was warmed up by hand, i.e. an increase of 0.6 bar occurred when the temperature increased with $8{ }^{\circ} \mathrm{C}$. These observations suggested the measured gas pressure most likely is influenced by the temperature of the stick and the ambient conditions. It was found that the room temperature, which was controlled by an air conditioning system, was still varying. These ambient temperature fluctuations during the recorded reactions caused periodical variations of the gas pressure in the pVT system (Fig. 5a). Together with the pressure, values of the ambient temperatures were recorded. As it is shown with a part of one reaction (Fig. 5a) a correlation between the 24-hour trend of the room temperature and the measured gas pressure was observed. For example, pressure variations of 
about 0.6 bar at a change of the ambient temperature of about $2 \mathrm{~K}$ for a period of $8 \mathrm{~h}$ were recorded. They could not come from the measuring system, which possesses a high accuracy according its characteristics, given by the manufacturer (see above description of the pressure sensor). The sensor drift of the measured values with time is less than a fraction of percent per year and also cannot explain the observed fluctuations. Thus, as it was mentioned before, the source of the recorded pressure variations had to be searched in the thermodynamic behavior of the gas in the stick. Even in a small capillary volume of the stick, neglected with respect to the cell volume, the gas underwent a big temperature gradient at a large contacting surface with the ambient atmosphere. During its pathway to the sample the income gas passed regions with nonconstant atmosphere temperature of the stick environment causing a gas expansion or a shrinking. Thus, the variable gas pressure in the capillary influenced the record during the reactions and could not be neglected. There were two possible ways to deal with the problem: one was to find a way to correct the pressure record for the change of the ambient temperature; and the other was to improve the set-up in order to reduce the volume of the gas exposed to the ambient conditions. A procedure to correct the recorded gas pressures for the fluctuations of the ambient temperature was developed together with Georgi Genov (GZG-Göttingen). It is explained in details in Appendix C. The ambient temperature, necessary for the corrections, was measured at one point, close to the pressure gauge. To obtain the temperature variations along the whole pressure stick, i.e. to measure the stick temperature profile with time, is not a trivial task to be done. Thus, the pressure corrections for one point of the stick were multiplied by a factor of $\gamma$ in order to gain the pressure corrections for the whole stick. The values of $\gamma$ were determined by eye when the curve of the corrected pressure drop became smoothed (Fig. 5a). Figure $5 \mathrm{~b}$ shows an example how one correction of the pressure for the ambient temperature smoothes the obtained curve of the consumed pressure. 

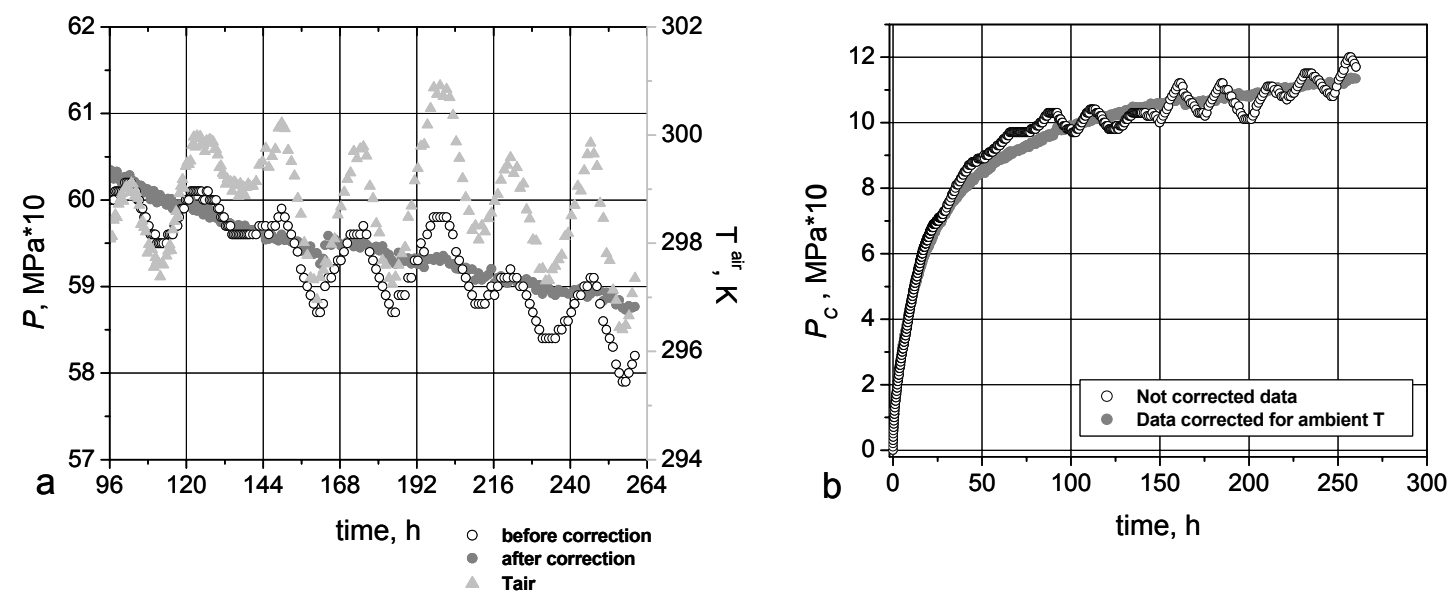

Figure 5. Pressure recorded during a growth of $\mathrm{CH}_{4}-\mathrm{D}_{2} \mathrm{O} @ 6.0 \mathrm{MPa},-5^{\circ} \mathrm{C}$ (a) showed variations which correlated quite well with a 24-hour changes of the ambient temperature. An applied data correction using a factor $\gamma$ of 1.5 (see the text) smoothed the pressure. It improved the corresponding kinetic curve (b) as it is shown with the plot of the consumed gas calculated from the corrected (grey circles) and non-corrected (white circles) pressures.

The reproducibility of the gas-consumption (pVT) measurements with the set-ups described above was checked. Kinetic runs of $\mathrm{CH}_{4}$-hydrate formation were done at identical thermodynamic conditions with $\mathrm{D}_{2} \mathrm{O}$ - or $\mathrm{H}_{2} \mathrm{O}$ ice-gas systems and a good agreement between the results obtained by both set-ups was obtained (see Fig.6).
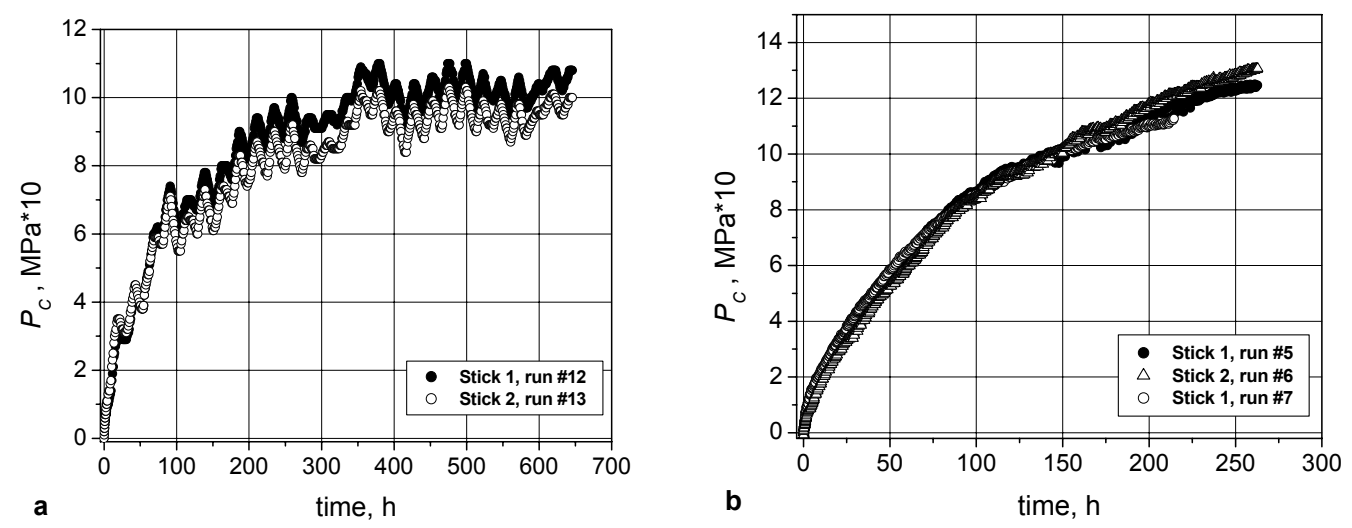

Figure 6. Plots of the methane clathrate reactions measured by both pVT set-ups. Formations of (a) $\mathrm{H}_{2} \mathrm{O}-\mathrm{CH}_{4}$ (a) 6.0 Mpa, $-3^{\circ} \mathrm{C}$ and (b) $\mathrm{D}_{2} \mathrm{O}-\mathrm{CH}_{4} @ 6.0 \mathrm{MPa},-10^{\circ} \mathrm{C}$ are shown (see also Table 1, Ch. 3.3.4).

The ambient temperature fluctuations caused an oscillatory behavior of the consumed pressure at a temperature of $-3^{\circ} \mathrm{C}$ recorded simultaneously in both set-ups during the summer runs. The later kinetic runs at $-10^{\circ} \mathrm{C}$ were corrected for the ambient temperature fluctuations and showed a good 
reproducibility of the recorded reactions - the winter runs (simultaneous records in both set-ups, \#5 and \#6) and the summer one (run \#7).

\subsubsection{Sample Recover and Measurement}

The non-stoichiometry of the clathrate structures (Sloan, 1998) makes difficult to determine the amount of gas hydrate at an absolute scale using the number of encaged gas molecules. In addition the absolute volume of the set-up is not known and the gas law cannot be used to get the number of the trapped gas molecules. Thus, an X-ray measurement of each quenched sample would give the amount of gas hydrate relative to the one of ice, left from the reaction.

The penetration power of the laboratory X-ray sources was not enough to perform the measurement of the sample under pressure. Thus, a sample recovery to liquid nitrogen was necessary to avoid the hydrate decomposition at ambient pressure. Consequently, in order to be measured by X-ray, the gas hydrate sample had to be moved from one point to another within its stability field. Initially the following procedure was used (procedure 1): when a reaction was stopped the pressure cell was cooled in liquid nitrogen for few minutes. Then the gas pressure was released and the Bridgman seal was opened. The unscrewing of the seal took few minutes because of the deep temperature reached by the pressure cell. The sample was recovered and then stored at liquid nitrogen temperature. The disadvantage of this procedure was that the low temperatures, required by the hydrate stability at ambient pressure (below 190K), made the opening of the Bridgman seal very difficult. During this manipulation the thermodynamic conditions of the hydrate were not controlled well when the sample was taken out of the pressure cell. As a result some of the samples, recovered in this way, accidentally were moved out from the hydrate stability field and partly decomposed (see Ch. 3.3.5). For the later runs a new procedure (procedure 2) was used to recover the samples to the liquid nitrogen temperature. When the stick was taken out of the bath the gas pressure was maintained. Then under pressure the Bridgman seal was opened within about ten second and the sample was recovered in liquid nitrogen.

Before the X-ray diffraction measurements of the recovered samples, they were crushed in liquid nitrogen and filled in a sample holder already cooled in a liquid $\mathrm{N}_{2}$. To avoid the entering of frost into the sample the whole procedure was done for few minutes in a cold room at a temperature of $-10^{\circ} \mathrm{C}$. Each recovered sample was then investigated at $80 \mathrm{~K}$ in a custom-made 
Philips MRD diffractometer equipped with an APD helium closed-cycle cryostat. X-ray histograms were measured at three $\omega$-positions of the sample. They were used to obtain the hydrate weight fraction (alpha) with a precision of $\pm 3 \%$, given by the data processing and the difference in the measured histograms (Evgeny Goreshnik-GZG, Göttingen, private communications).

For each kinetic run a ratio $\alpha / P_{c}$ of the hydrate fraction of the quenched sample to the total consumed gas was multiplied by the gas-consumption data. Thus, the values of the clathrate fraction were deduced as a function of time.

\subsubsection{Gas-Consumption Data of $\mathrm{CH}_{4}$-clathrate Growth}

The gas-consumption method described above was used to study the long-term methane clathrate growth, lasting typically few weeks. The influence of the thermodynamic conditions (pressure and temperature) and the isotopic difference between $\mathrm{H}_{2} \mathrm{O}$ and $\mathrm{D}_{2} \mathrm{O}$ ice on the rate of $\mathrm{CH}_{4}$ clathrate formation was investigated. Table 1 shows the conditions, the set-ups and the durations of the reactions studied by the pVT method. Information about the applied temperature correction, the recovery procedure and the X-ray measurement of the reacted samples are also given for each kinetic run.

A fast grain growth of the ice at temperatures close to the melting point made it difficult to perform high temperature studies of the methane clathrate growth. A fast decrease of the initial ice surface hindered or even suppressed the nucleation of $\mathrm{CH}_{4}$-hydrate and its following growth. In order to proceed clathrate formation runs at high temperatures a hydrate was initially formed at a low temperature. The formed hydrate cover on each ice grain prevents any fast ice growth in this case. Then it was used to run high temperature reactions. Samples were led to react at 6.0 $\mathrm{MPa}$ and $-7^{\circ} \mathrm{C}$ for $4 \mathrm{~h}$ to form the initial hydrate. Then these samples were used as a starting material for kinetic runs at temperatures of -5 to $-1^{\circ} \mathrm{C}$ (see Table 1$)$. 


\begin{tabular}{|c|c|c|c|c|c|c|c|c|c|c|}
\hline Run & $\begin{array}{c}\text { Stick } \\
\text { No }\end{array}$ & $\begin{array}{c}\text { Starting } \\
\text { Ice/Clathrate }\end{array}$ & $\begin{array}{l}\mathbf{T}, \\
\mathbf{K}\end{array}$ & $\begin{array}{l}\text { P(f), } \\
\text { MPa }\end{array}$ & $\begin{array}{c}\mathbf{P}_{d}\left(\mathbf{f}_{d}\right) \\
\mathbf{M P a}\end{array}$ & $\begin{array}{c}\text { Excess } \\
\text { fugacity, } \\
\left(\mathbf{f}-\mathbf{f}_{\mathrm{d}}\right) / \mathbf{f}_{\mathrm{d}}\end{array}$ & $\begin{array}{c}\text { Duration, } \\
h\end{array}$ & $\begin{array}{c}\text { Correct. } \\
\gamma\end{array}$ & $\begin{array}{c}\text { Recover } \\
\text { procedure } \\
\text { No } \\
\end{array}$ & X-ray \\
\hline$\# 1$ & 2 & $\mathrm{H}_{2} \mathrm{O}$ & 245 & $6.0(4.87)$ & $1.03(0.996)$ & 3.89 & 839 & - & 1 & yes \\
\hline$\# 2$ & 1 & $\mathrm{D}_{2} \mathrm{O}$ & 253 & $6.0(4.99)$ & $1.34(1.29)$ & 2.87 & 340 & 1.0 & 2 & yes \\
\hline$\# 3$ & 2 & $\mathrm{H}_{2} \mathrm{O}$ & 258 & $6.0(5.05)$ & $1.57(1.50)$ & 2.37 & 215 & 1.5 & 1 & yes \\
\hline$\# 4$ & 1 & $\mathrm{H}_{2} \mathrm{O}$ & 263 & $6.0(5.11)$ & $1.84(1.75)$ & 1.92 & 142 & - & 1 & yes \\
\hline$\# 5$ & 1 & $\mathrm{D}_{2} \mathrm{O}$ & 263 & $6.0(5.11)$ & $1.84(1.75)$ & 1.92 & 263 & 1.0 & 2 & yes \\
\hline$\# 6$ & 2 & $\mathrm{D}_{2} \mathrm{O}$ & 263 & $6.0(5.11)$ & $1.84(1.75)$ & 1.92 & 263 & 1.0 & 2 & yes \\
\hline$\# 7$ & 1 & $\mathrm{D}_{2} \mathrm{O}$ & 263 & $6.0(5.11)$ & $1.84(1.75)$ & 1.92 & 214 & 1.7 & 1 & yes \\
\hline$\# 8$ & 1 & $\mathrm{D}_{2} \mathrm{O}$ & 263 & $3.0(2.77)$ & $1.84(1.75)$ & 0.58 & 506 & $1.0^{(1)}$ & 2 & yes \\
\hline$\# 9$ & 2 & $\mathrm{D}_{2} \mathrm{O}$ & 263 & $9.0(7.07)$ & $1.84(1.75)$ & 3.04 & 506 & $1.0^{(1)}$ & 2 & yes \\
\hline$\# 10$ & 1 & $\mathrm{D}_{2} \mathrm{O}$ & 268 & $6.0(5.16)$ & $2.19(2.07)$ & 1.49 & 260 & 1.5 & 1 & yes \\
\hline$\# 11 *$ & 2 & $\mathrm{H}_{2} \mathrm{O}-\mathrm{CH}_{4}$ & 268 & $6.0(5.16)$ & $2.19(2.07)$ & 1.49 & 333 & 1.7 & 1 & no \\
\hline$\# 12 *$ & 1 & $\mathrm{H}_{2} \mathrm{O}-\mathrm{CH}_{4}$ & 270 & $6.0(5.19)$ & $2.33(2.20)$ & 1.36 & 646 & - & 1 & yes \\
\hline$\# 13 *$ & 2 & $\mathrm{H}_{2} \mathrm{O}-\mathrm{CH}_{4}$ & 270 & $6.0(5.19)$ & $2.33(2.20)$ & 1.36 & 646 & - & 1 & yes \\
\hline$\# 14 *$ & 1 & $\mathrm{H}_{2} \mathrm{O}-\mathrm{CH}_{4}$ & 271 & $6.0(5.19)$ & $2.41(2.28)$ & 1.28 & 119 & - & 1 & no \\
\hline$\# 15 *$ & 2 & $\mathrm{H}_{2} \mathrm{O}-\mathrm{CH}_{4}$ & 271 & $6.0(5.19)$ & $2.41(2.28)$ & 1.28 & 119 & - & 1 & yes \\
\hline$\# 16 *$ & 2 & $\mathrm{H}_{2} \mathrm{O}-\mathrm{CH}_{4}$ & 272 & $6.0(5.20)$ & $2.50(2.36)$ & 1.20 & 165 & - & 1 & yes \\
\hline
\end{tabular}

All gas-consumption runs used starting ice powder with a mean radius of $27 \mu \mathrm{m}$.

* Runs, performed at high temperatures using ice grains covered initially by methane hydrate (see the text).

Table 1. Detailed information about the $\mathrm{CH}_{4}$-gas hydrate formations studied by the gas-consumption method. The last two columns give information about the recovery procedure of the quenched samples and whether they are measured by X-ray or not. Some of the kinetic data were corrected for ambient temperature fluctuations (column marked as "Correct.") and a correction factor $\gamma$ is given (see also Fig. 5) A problem in the serial communication of the temperature sensor after first $270 \mathrm{~h}$ of reaction and a non-recoverable temperature record allowed only a partial correction of the two data sets denoted by a superscript (1). 


\subsubsection{Data Comparison}

To check for consistency of the kinetic results obtained by the gas-consumption and neutron diffraction method both were compared with each other. The kinetics of methane clathrate formation at pressure of $6.0 \mathrm{MPa}$ and two different temperatures, starting from deuterated ice powder, was recorded by neutron diffraction and gas consumption (Fig.7). The comparison of the results obtained by both methods showed that the reactions observed in the laboratory developed slightly faster. The methane gas, reacting with the ice sample, was cooled along its way through the stick capillary for about $100 \mathrm{~cm}$ (within cryostat chamber) and $5 \mathrm{~cm}$ (within bath) at the setup used for the neutron diffraction and the gas consumption, respectively. The reactions, observed by the pVT method, used a gas experiencing a strong temperature gradient within the stick capillary from ambient temperature to the one of the reaction. As a result the obtained kinetics could slightly differ from the one recorded in the cryostat.
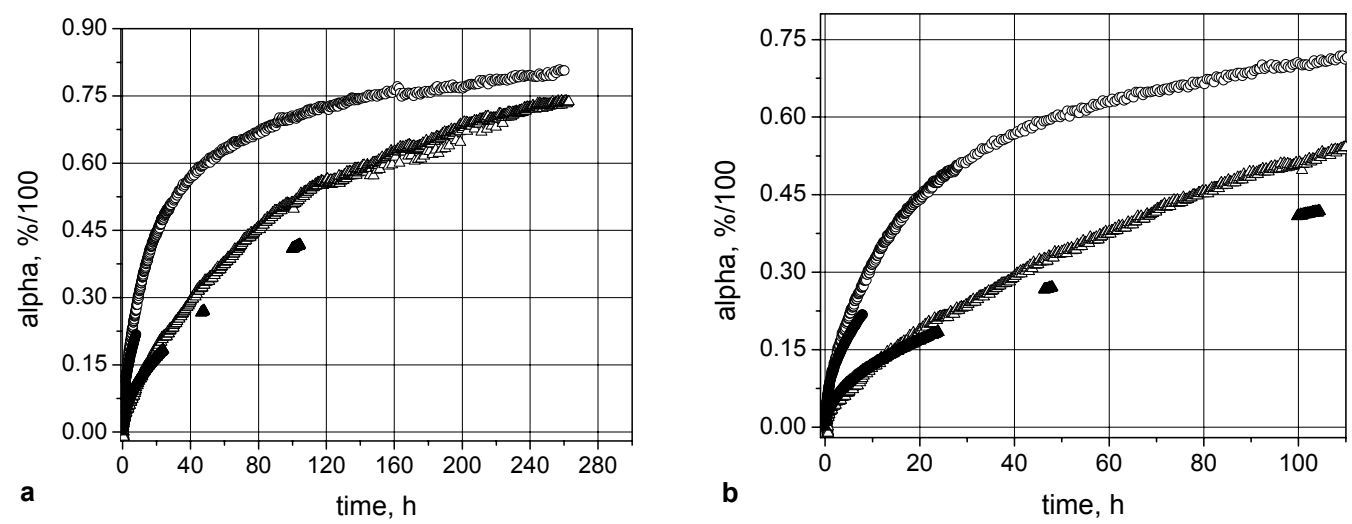

Figure 7. Comparison of $\mathrm{D}_{2} \mathrm{O}-\mathrm{CH}_{4}$ hydrate kinetic runs (a) monitored by a neutron diffraction- (black symbols) and a pVT-method (white symbols). A magnification of the initial reaction kinetics from (a) is shown in (b). The reactions at temperatures of $-5^{\circ} \mathrm{C}$ and $-10^{\circ} \mathrm{C}$ are marked by circles and triangles, respectively.

As it was described in Ch. 3.3.3 the gas-consumption method required a recovery of the quenched samples. As a result of the recovery procedure some of the samples, reacted at pressure of 6.0 Mpa, were decomposed and their gas-consumption data could not be converted to clathrate fraction data. The samples formed at 6.0 MPa (which did not decompose) were used to obtain a calibration for the gas-consumption data recorded with both pVT set-ups. The ratios of the measured clathrate fractions $\alpha$ to the related consumed amounts of gas $P_{C}$ were plotted versus temperature (Fig. 8). The points, corresponding to the decomposed samples, were excluded in the linear fit of the data and $\alpha / P_{C}$ was deduced as a function of the temperature. 


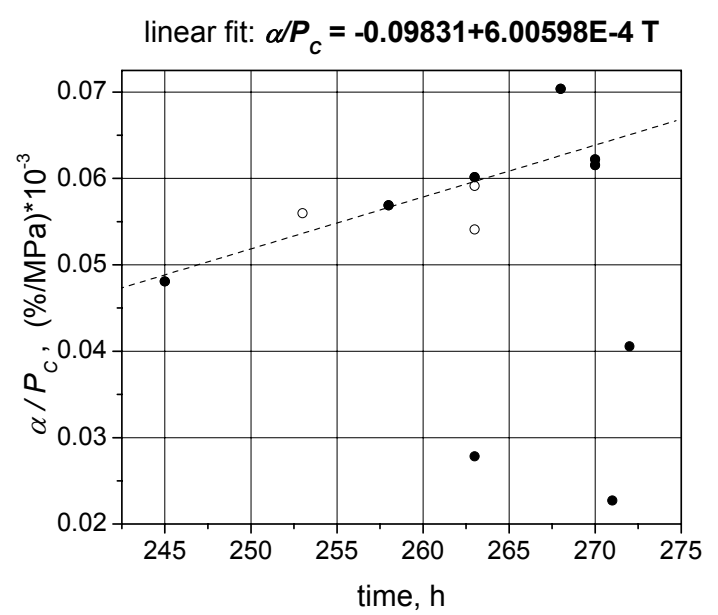

Figure 8. Ratio of the formed hydrate $\alpha$ to the consumed gas $\boldsymbol{P}_{C}$ is plotted as a function of the temperature. When procedure 1 was used to recover the samples (black circles) three of them were decomposed that gave very low values of $\alpha$. Afterwards procedure 2 was implemented (white circles). The $\alpha / P_{C}$ ratios of the decomposed samples was obtained from a linear fit of the other ratios and the lost quantities of hydrate were recovered (see the discussions in the text).

The obtained mathematical expression was used to calibrate the gas-consumption data of each decomposed sample and to deduce the corresponding gas hydrate kinetics (Fig. 9). Kinetic runs of samples recovered by both procedures were compared and a disagreement between the results of the gas consumption and the hydrate fraction was observed. The kinetics of the $\mathrm{CH}_{4}$ gas hydrate (\#5, Table 1) was used as a reference one because of the reliable recovery procedure used for the sample quenching. For each decomposed sample the calibration function (Fig.8) was used to convert the amount of consumed gas to an amount of hydrate. 

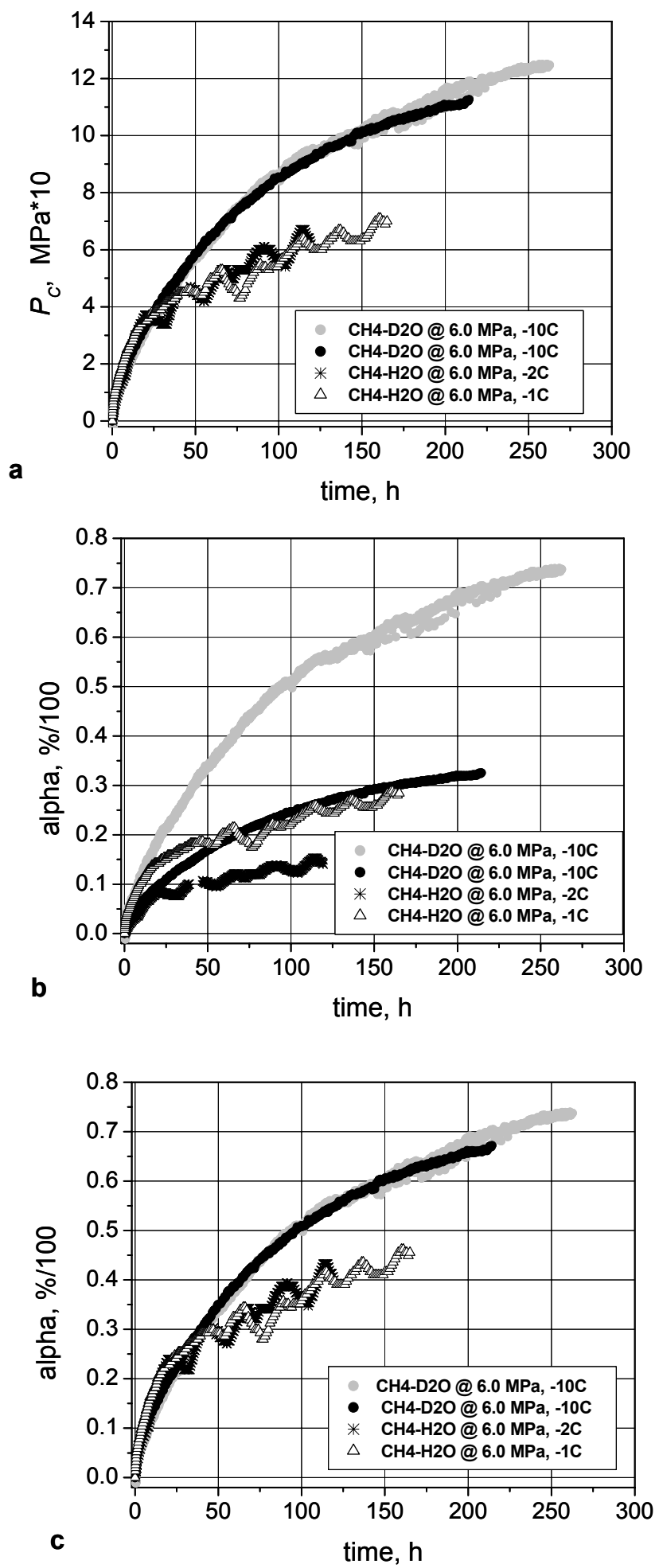

Figure 9. The gas consumptions obtained (a) during the clathrate formation runs \#5, \#7, \#15, \#16 (see Table 1) were compared with the hydrate fractions of the quenched samples (b) recovered by procedure 2 (grey symbol) or 1 (black symbols). The obtained low values of clathrate fraction (see also Fig. 8) resulting from the sample decomposition were corrected (c) using the amount of consumed gas. 
Clathrate growths of quenched samples, which were not measured by X-rays (see Table 1), were also obtained from recorded gas consumptions using the formula described above (see Fig.10).
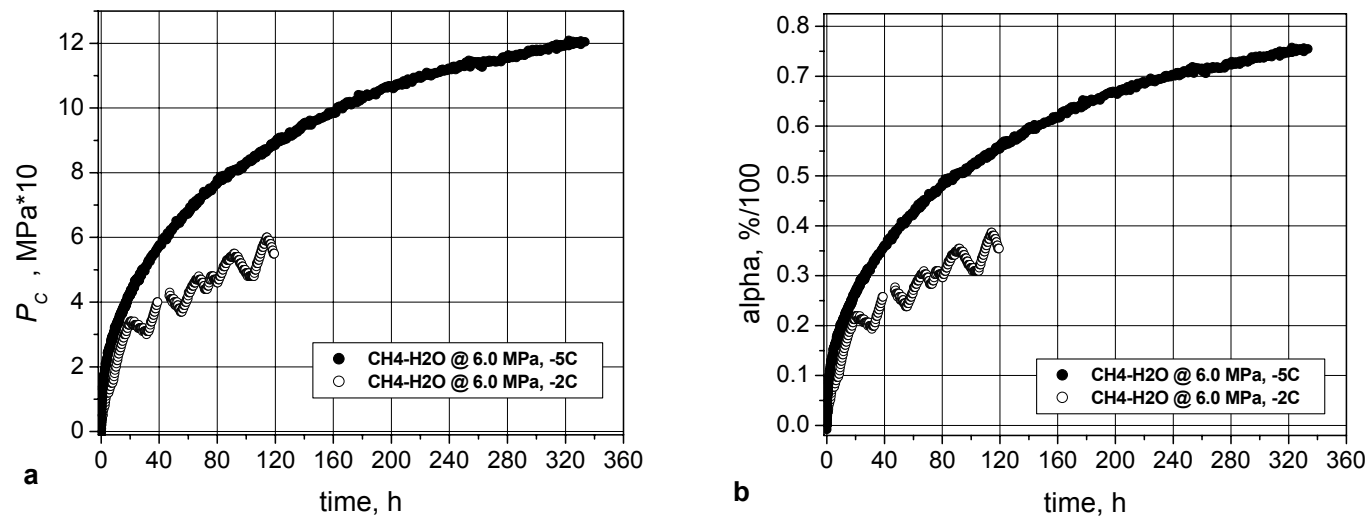

Figure 10. Gas consumed during gas hydrate formation reactions (a) can be used to obtain the amount of formed hydrate (b) in case of an absence of X-ray measurements of the quenched samples. Data of $\mathrm{CH}_{4}-\mathrm{H}_{2} \mathrm{O}$ gas hydrate growth at pressure of 6.0 MPa and temperatures of $-5^{\circ} \mathrm{C}$ and $-2^{\circ} \mathrm{C}$ are marked with black and white circles, respectively.

The observed oscillatory behavior of the measured gas consumption was not corrected for the ambient-temperature fluctuations because they were not recorded at that time. 


\subsection{FE-SEM Method}

Field Emission Scanning Electron Microscopy (FE-SEM) was used to study the microstructural aspects of the formation of gas hydrates starting from a well-characterized ice powder under controlled pressure and temperature conditions.

\subsubsection{Physics of the Scanning Electron Microscopy}

Scanning electron microscopy (SEM) can image and analyse the surface of three-dimensional objects. It uses electrons as a source for an image formation. The SEM work-principle is based on the de Broglie-wave character of electrons and their charge. As electronegative particles the electrons can be focused by means of a magnetic or an electrostatic field. When an electron beam reaches a target sample several electron-sample interactions can occur and can give information about the target. The most important interaction processes are schematically shown in Figure 1.

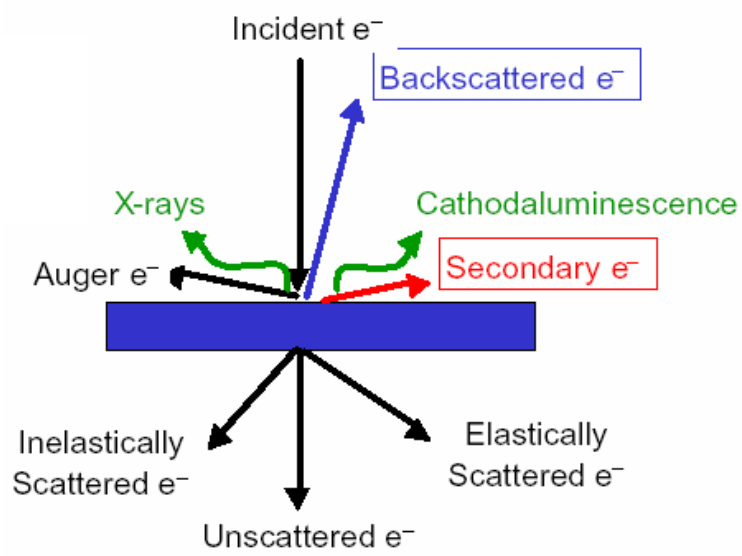

Figure 1. Schematic drawing of the elementary atomic interaction processes between an incident electron and a target sample.

Depending on their origin the electrons, coming from the beam-specimen interactions, can be grouped in several types. When an incident electron collides with an atom in the sample a deflection of the electron's path, known as Rutherford elastic scattering, occurs. Incident electrons, which are completely backscattered from the specimen surface, are called backscattered electrons. Since the scattering angle and the backscattered electron yield are strongly dependent on the atomic number of the involved nucleus, the detected backscattered electrons yield images with compositional information for the sample. 
When a high-energy electron passes close to the atoms in the specimen, it can give some of its energy to lower-energy electrons of the atomic shells. As a result secondary electrons are produced. The amount of energy given to the secondary electrons is very small and thus only those of them, which are produced within a very thin surface layer (less than $10 \mathrm{~nm}$ thick), are able to escape from the sample. The detection of these secondary electrons provides highresolution topographical images.

When a secondary electron leaves the inner atomic shell a vacancy is produced. Its filling with a higher energy electron from the same atom will cause an energy release either via an emitting of an outer electron (Auger electron) or by an emitting of an X-ray. Each auger electron has a characteristic energy corresponding to the element from which it is emitted and brings information about the specimen composition. The characteristic lines of an X-ray spectrum correspond to the electronic transitions involved in the energy release. Since these lines are specific for a given element, the composition of the material can be deduced. This can be used to provide a quantitative information about the elements present at a given point of the sample, so called Energy-dispersive analysis of X-rays (EDX), or alternatively to map the amount of a particular element as a function of position.

Cathodoluminescence (CL) or the emission of an ultraviolet, a visible or an infrared light is caused by an electron bombardment of semiconductors and mineral samples. In combination with an EBIC (Electron-Beam-Induced Current) in semiconductors, CL is used to image lattice defects because of the recombination of charge carriers at the lattice defects.

Unscattered electrons are incident electrons, which are transmitted through a thin specimen without any internal interaction. Incident electrons, which interact with the atoms of the sample in accordance with Bragg's law (no energy loss) and as a result deflect from their initial path, are called elastically scattered electrons. In contrast inelastically scattered electrons are the incident electrons, which loose their energy in the interaction with the specimen atoms. Absorption of incident electrons by the sample can make it a charged object. This phenomenon is known as a "charging" of the sample.

The backscattered and secondary electrons are the most important signals for an image recording. The backscattered electrons provide a good compositional imaging of the sample, while the secondary electrons produce better topographical images. 


\subsubsection{FE-SEM Instrument - Description and Image Output}

Ex situ studies on gas hydrates were made using a high-resolution cryo field-emission scanning electron microscope type LEO 1530 Gemini installed at GZG, Abt. Angewandte Geologie (Figure 2). It is equipped with a cryo transfer unit (Oxford instruments), which permits SEM measurements at low temperatures to be made. Dry $\mathrm{N}_{2}$-gas, taken from a Dewar filled with liquid nitrogen at $-190^{\circ} \mathrm{C}$, is used to cool a cryo stage. The temperature of the cryo stage is controlled by the gas flow and the level of liquid nitrogen in the Dewar.

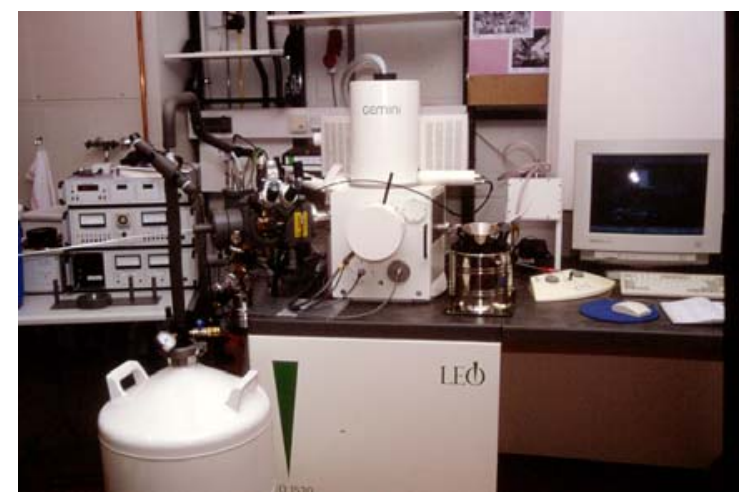

Figure 2. Picture of the cryo field-emission scanning electron microscope (LEO1530) used for the gas hydrates studies. The photo is provided by K.Techmer, GZG, Kristallographie.

The main principle of the FE-SEM instrument is shown in Figure 3. The electrons, coming from a Schottky cathode, are pulled towards the specimen surface by means of applied potentials $\mathrm{V}_{0}$ and $V_{B}$ and focused on it by a GEMINI column lens system. A beam booster maintains high beam energy throughout the whole electron optical column. An electromagnetic multi-hole beam aperture changer is incorporated close to the electron source. In conjunction with the magneticfield lens, this is used to select an optimum beam aperture angle and to tune the probe current. The electron beam scans the specimen in a raster controlled by small coils of wire carrying the controlling current (scan coils). The details about the LEO 1530 microscope and the working conditions, at which the FE-SEM images on gas hydrates are made, are described in the following sections. 


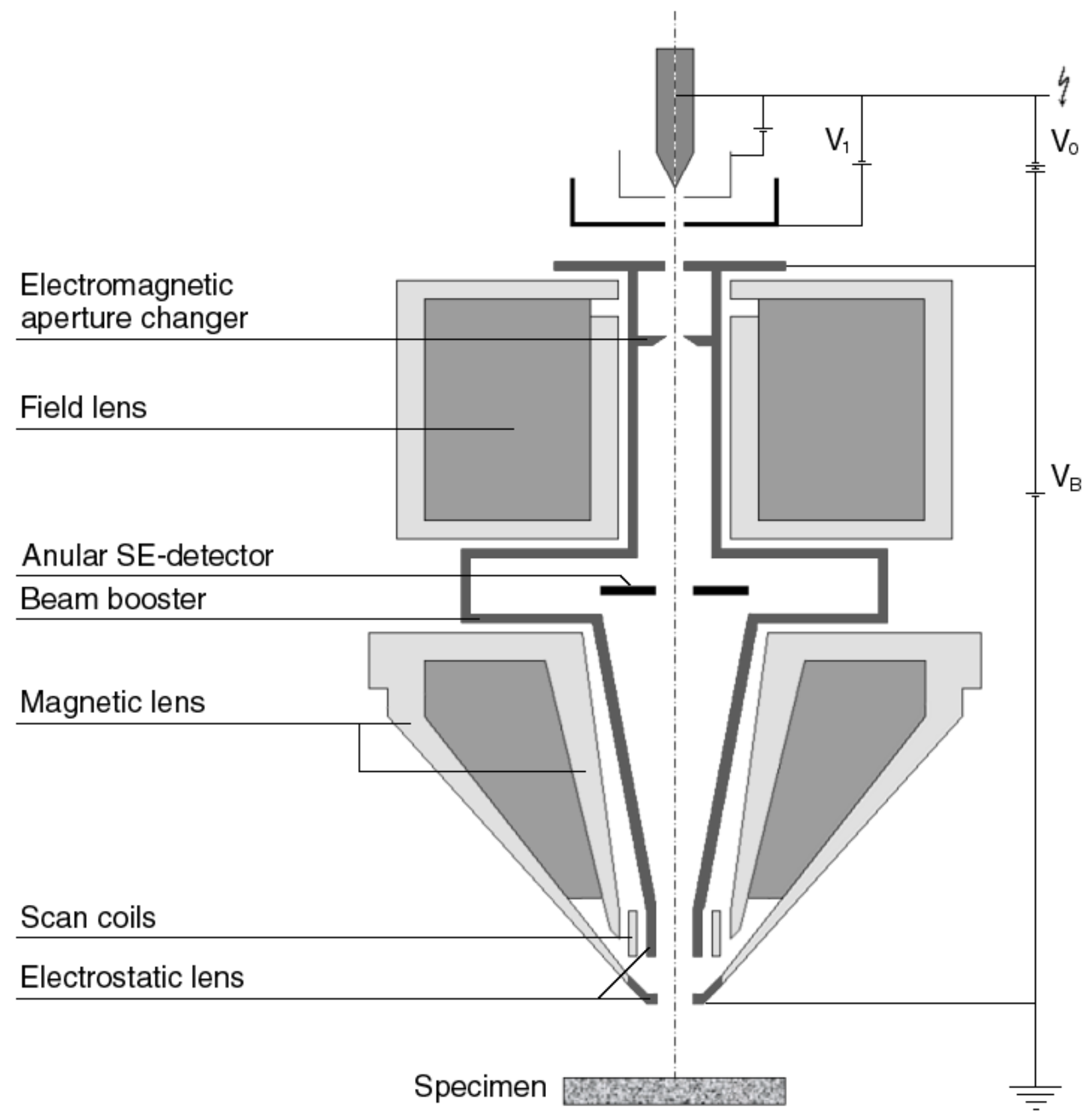

Figure 3. Schema of the electron optics of a LEO 1530 Gemini electron microscope. The potentials $V_{0}, V_{1}$ and $V_{B}$ denote an applied extractor voltage at the first anode, an accelerator voltage at the second anode and a booster voltage, respectively. Original pictures were provided by Till Heinrichs, GZG, Abt. Angewandte Geologie. 
Electrons from a hot Schottky field-emission cathode are extracted and accelerated by means of potential differences between the cathode tip and the first and the second anode, respectively. The primary electrons are produced by $\mathrm{ZrO} / \mathrm{W}(100)$-type or Schottky-emission cathode (Figure 4).
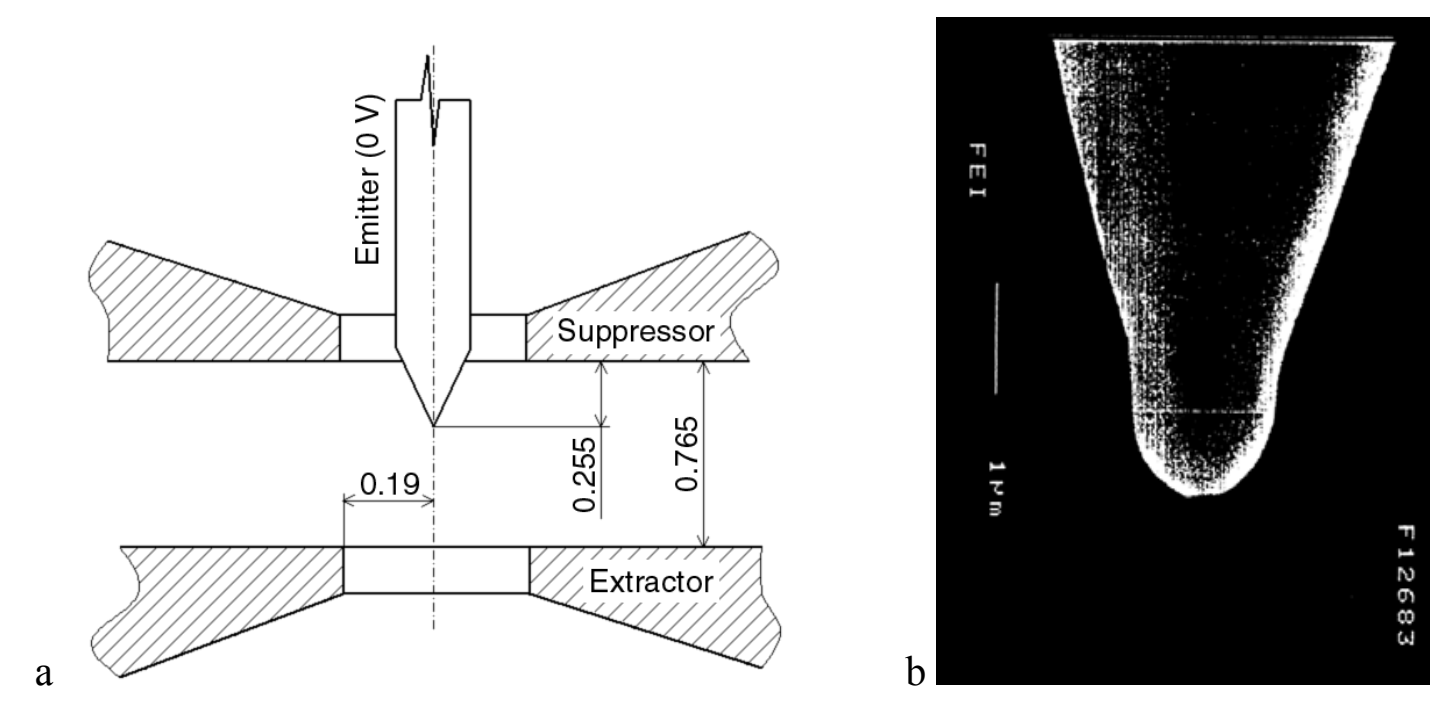

Figure 4. Cross-section of Schottky type field-emission gun (a) with an emitter tip shown on a SEM picture (b). Original pictures were provided by Till Heinrichs, GZG-Göttingen.

The potential barrier or the minimum energy required for an electron to leave the surface of the tip is lowered by a $\mathrm{ZrO}$ coating from $4.5 \mathrm{eV}$ (in a thermionic tungsten emitter) to $2.8 \mathrm{eV}$ (in a $\mathrm{ZrO} / \mathrm{W}$ emitter). The electrons are extracted by an application of a voltage of $5 \mathrm{kV}$ to an extractor anode. Selected (100) crystallographic orientation of the tip concentrates the emission within a cone with a semi-apex angle of about $0.1 \mathrm{rad}$ as a result of the dependence of the potential barrier on the surface orientation of the tip. A second anode accelerates the emitted electrons. The brightness of the field-emission gun is $5 \cdot 10^{8} \mathrm{~A} / \mathrm{cm}^{2}$ sr that is about 100-1000 times higher than the one of the conventional thermionic gun $\left(\sim 10^{5}-10^{6} \mathrm{~A} / \mathrm{cm}^{2} \mathrm{sr}\right)$. The size of $10-20 \mathrm{~nm}$ of the crossover (the smallest beam cross-section) of the $\mathrm{ZrO} / \mathrm{W}$ Schottky type gun is 1000 times smaller than the one of the tungsten thermionic gun $(20-50 \mu \mathrm{m})$. The field emission cathode is surrounded by a negative biased suppressor electrode, which collects the dispersed electrons with a kinetic energy lower than the one of the extracted electrons. In order to avoid the cathode tip to be destroyed by an ion bombardment from the residual gas an ultrahigh vacuum of $10^{-9}$ mbar (UHV) is applied. The vacuum is also necessary to provide an unobstructed travel of the electron 
beam from the electron gun down the column and to the target sample. Moreover, vacuum conditions are necessary for the resulting secondary electrons because their energy is lower than the one of the incident electron beam and their paths could easily be altered by the atmospheric atoms, possibly skewing the analysis results.

The electron beam has a transverse chromatic aberration (an enlargement of the focal spot), caused by a spread of electron energy when the beam is scanned in the deflection system. This effect can decrease the resolution of the outer areas of an image. LEO 1530 is equipped with a Gemini lens (magnetic and electrostatic lens, Figure 3), specially designed to diminish the chromatic aberration by a diminishing of the electron beam energy. They are made as an analog of the optic achromatic lens, consisting of two component lenses, one convergent and the other one - divergent. A working distance is defined as the distance between the electrostatic lenses and the specimen. With an increase of the distance the observed surface area increases together with the spherical aberration, resulting in a blurred image. With decrease of the working distance the spherical aberration will decrease. An optimum distance from the specimen, providing an optimum observed area and a low spherical aberration, has to be found. At a position of the specimen closer to the front lenses a higher magnification of the observed object can be obtained. The magnification in the SEM results is the ratio of the area of the screen showing the image to the area scanned on the specimen. An increase of the magnification is therefore achieved by scanning the electron beam over a smaller area of the specimen.

The FE-SEM images are obtained by the secondary electrons. They are detected by a scintillator-photomultiplier combination known as a standard Everhart-Thornley detector. The secondary electrons are collected by a grid-biased $+350 \mathrm{~V}$ and accelerated to the scintillator. The interaction of the secondary electrons with the scintillator causes an emission of photons. This light is guided to a photomultiplier tube, which converts the photons of light into a current. The current depends on the number of secondary electrons hitting the scintillator. By a measuring of this current a point of a relevant gray-scale value is displayed on the graphic container control (Picture box) of the imaging software. Repeating this procedure for the whole observed area leads to the complete image build-up. After the scan is finished and the save command is entered the content of the Picture box is saved on the hard disk.

The tiny electron beam and the Gemini lenses permit the SEM images obtained at low $\mathrm{kV}$ to have high resolution and contrast. The improved contrast makes a better gray scale differentiation of the topology of the surface and more details are distinguished. A slow 
sublimation of the sample surface takes place when the sample stays in the vacuum more than half an hour. A quick sublimation within a defined area of the sample can be obtained with an increase of the beam accelerating voltage up to $2.5-3 \mathrm{kV}$ and an increase of the probe current from $50 \mathrm{pA}$ to $100-200 \mathrm{pA}$.

A charging of the surface is another effect, which can appear during the scanning. It depends on the sample and the length of time of scanning. The faster the scanning is, the lower is the charging. The topology of the surface also can lead to an observable charging, for example, in cases of sharp edges at the surface.

The LEO 1530 microscope was used to obtain FE-SEM images of gas hydrates which were of a major importance to deduce their microstructure.

\subsubsection{Image Data Collection}

Samples of ice powder, used as a starting material, and methane gas hydrate, formed in the laboratory (Göttingen), were stored in liquid nitrogen. The ice and clathrate samples were transferred to a pre-cooled cryo-stage of the LEO 1530 microscope via an interlock. The sample holder was cooled in liquid nitrogen and then transferred into the chamber with a droplet of nitrogen left in the sample space. The electron microscopic work was carried out at temperatures below $-165^{\circ} \mathrm{C}$ and no decomposition of the gas hydrate was observed. Once the UHV was obtained a low-voltage mode $(1.5-2 \mathrm{kV})$ for the primary electrons forming an electron-probe current of 20-50 pA at the specimen surface was applied. The value of the electron voltage was recorded as an EHT value in a comment field of each image. FE-SEM pictures on starting ice spheres as well as on grown methane hydrates were collected at a working distance of about 10-12 $\mathrm{mm}$. A distance of $6 \mathrm{~mm}$ was used when there was a need of higher magnification. The images were obtained typically at a slow scan rate (30s/frame). The charging of the specimen surface during the measurement appeared to be much smaller in case of the gas hydrate as compared to the ice. The ice powders used as a starting material and the gas hydrates obtained after reactions with a methane gas were investigated by the FE-SEM and series of about 20-30 images per sample loading were collected. 


\subsubsection{Starting Material Observations}

The morphology of the starting material was investigated by FE-SEM and the obtained images were used to make a quantitative analysis of the produced ice spheres. Figure 5 shows a typical image of the produced ice powder containing spheres obtained by the spraying technique (see Chapter 3.1.5). Some of the ice spheres showed a cracked surface. It was caused by the fast freezing of the sprayed water droplets in liquid nitrogen. Provoked by the resulting large temperature gradients the frozen water droplets cracked due to differences in thermal contraction.
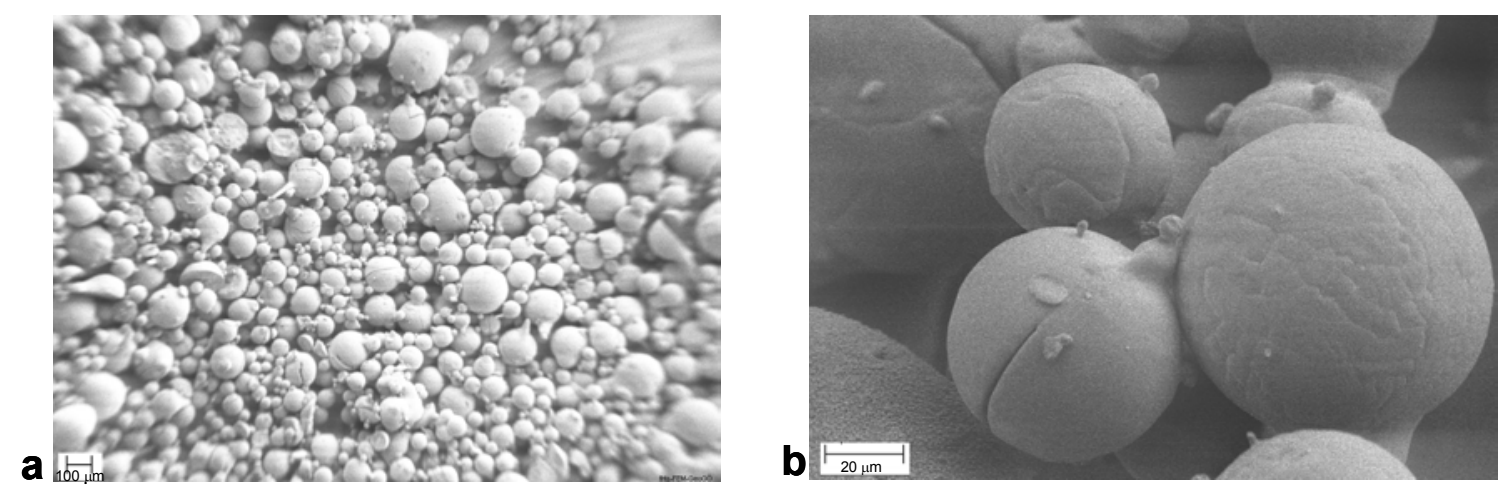

Figure 5. FE-SEM images, made on the starting material, show the initial hydrogenated-ice-Ih powder (a) consisting of ice spheres with an average diameter of 40-60 $\mu \mathrm{m}$ that can be distinguished at a larger magnification (b). Some of the ice grains have cracks caused by the method of their preparation.

The collected FE-SEM pictures were used to estimate a size distribution of the ice spheres produced by the spraying technique. A record of the diameters $d$ of about 1000 ice spheres per imaged sample was performed using software Image Tool and the results were statistically processed with Origin 6.0. Measurements on different batches showed that the size distribution of the ice spheres, sprayed with the same nozzle, was well reproducible and has a lognormal shape (Figure 6). It means that the plot of the grain diameters on a logarithmic scale $\ln (d)$ versus frequency of occurrence followed a normal distribution. From the fitted Gaussian a mean value and a standard deviation was calculated. 

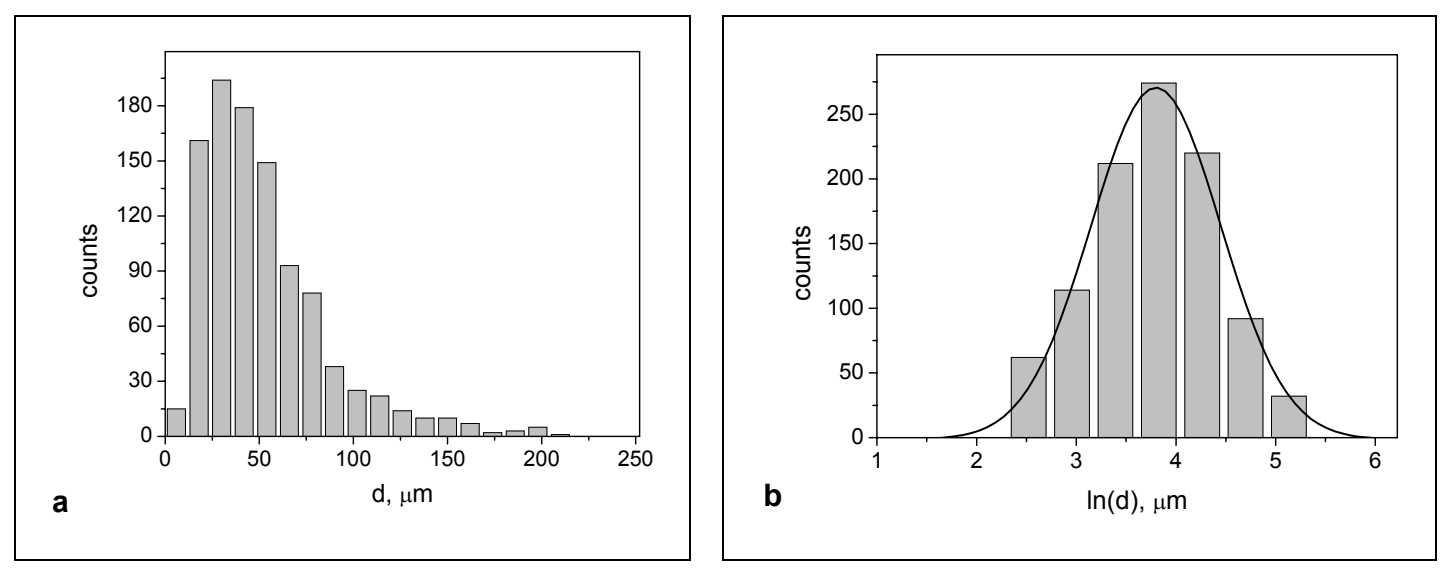

Figure 6. Histogram of the size distribution (a) obtained by a measuring of 1006 deuterated spherical ice grains imaged by FE-SEM. It converts to a histogram of a lognormal size distribution (b) when the grain diameters are plotted in a logarithmic scale $(\ln (d))$. The generated Normal distribution had a mean value $\langle\ln d\rangle$ of $3.8 \mu \mathrm{m}$ and a standard deviation $\sigma_{\ln (d)}$ of $0.65 \mu \mathrm{m}$ giving a mean grain size of $55.2 \mu \mathrm{m}$ (see Eq. 3 in the text).

When a normally distributed random variable $X$ is known, a lognormally distributed random variable $Y$ can be calculated by: $Y=e^{X}$. A mean $\langle Y\rangle$ and a standard deviation $\sigma_{Y}$ of the lognormal variable can be obtained from the mean $\langle X\rangle$ and the standard deviation $\sigma_{X}$ of the normal variable using the equations $1-2$

$$
\begin{aligned}
& \langle Y\rangle=e^{\langle X\rangle} \cdot e^{\sigma_{X}^{2} / 2} \\
& \sigma_{Y}=\langle Y\rangle \cdot \sqrt{e^{\sigma_{X}^{2}}-1}
\end{aligned}
$$

If the variable $X$ is replaced by a $\ln (d)$ and the variable $Y$ is replaced by $d$ the equations (1)-(2) transform to

$$
\begin{aligned}
& \langle d\rangle=e^{\langle\ln (d)\rangle} \cdot e^{\sigma_{\ln (d)}^{2} / 2} \\
& \sigma_{d}=\langle d\rangle \cdot \sqrt{e^{\sigma_{\ln (d)}^{2}}-1}
\end{aligned}
$$

where $\langle d\rangle$ and $\sigma_{d}$ are the mean and the standard deviation of a grain diameter calculated using the mean and standard deviation of the $\ln (d)$. The ratio between $\sigma_{d}$ and $\langle d\rangle$, obtained as

$$
\sigma_{0}=\frac{\sigma_{d}}{\langle d\rangle}=\sqrt{e^{\sigma_{\ln (d)}^{2}}-1}
$$

is called a relative standard deviation (Relative STD). 
Using of different nozzles, a powder of different particle size can be produced. Table 1 shows the mean values and the relative standard deviations obtained by the statistical processing of the FE-SEM images of four different batches of the starting ice powder.

\begin{tabular}{|c|c|c|c|}
\hline SeriesNo & Ice Ih & $\langle d\rangle$ & Relative STD, $\sigma_{0}$ \\
\hline 1 & $\mathrm{D}_{2} \mathrm{O}$ & 55.2 & 0.73 \\
\hline 2 & $\mathrm{D}_{2} \mathrm{O}$ & 52.3 & 0.87 \\
\hline 3 & $\mathrm{H}_{2} \mathrm{O}$ & 52.6 & 0.45 \\
\hline 4 & $\mathrm{H}_{2} \mathrm{O}$ & 69.7 & 1.0 \\
\hline
\end{tabular}

Table 1. Average grain diameter and its relative standard deviation obtained from different series of ice powder used in the kinetic experiments. The batches 1-3 were made with a different nozzle than the one used for 4 showing a starting material of a different mean diameter can be produced with different nozzles. The low value of $\sigma_{0}$ obtained for 3 is result of a low $\sigma_{\ln (d)}$ obtained from the lognormal distribution.

\subsubsection{Microstructural Observations}

FE-SEM images of gas hydrates showed an unique ability of the clathrates to form crystals of few tens of micrometers with a submicron porous microstructure (Figure 7).
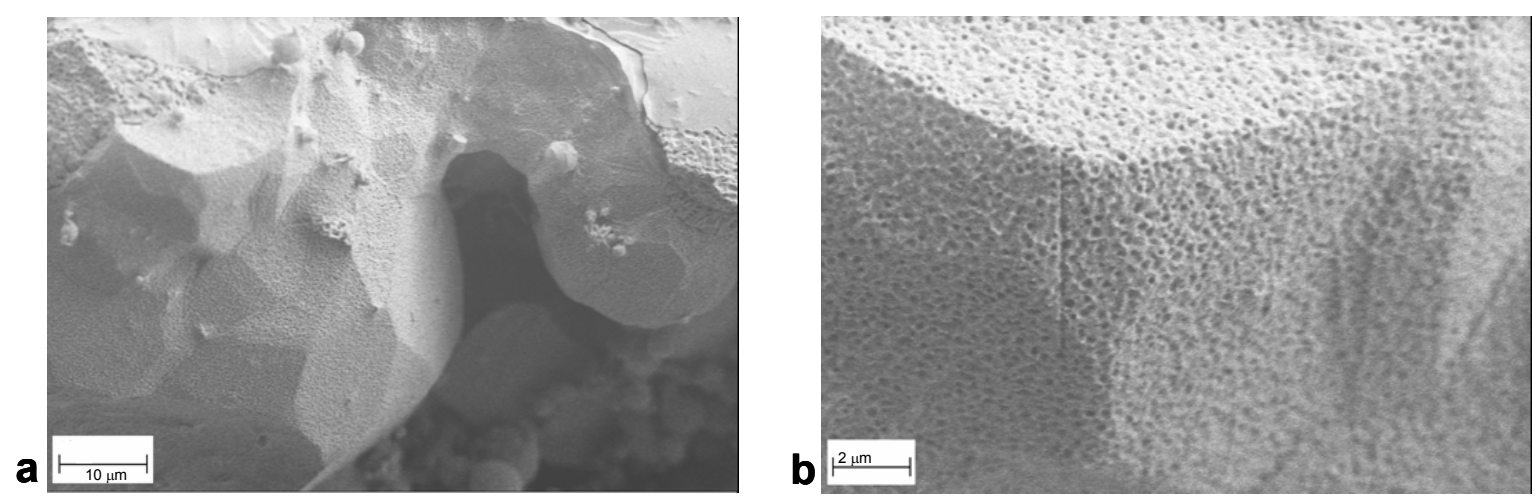

Figure 7. Artificial $\mathrm{H}_{2} \mathrm{O}$-methane gas hydrate, formed for 2 weeks of reaction at $6.0 \mathrm{MPa}$ and $264 \mathrm{~K}$. The typical hydrate crystallites grown from ice (a) were rather small $(20-30 \mu \mathrm{m})$ and showed a clear submicron porous structure at a higher magnification (b).

Methane and Carbon dioxide form gas hydrates with observed pores of a mean size in the order of several $100 \mathrm{~nm}$ (macropores) and several $10 \mathrm{~nm}$ (mesopores), respectively (Figure 8). 

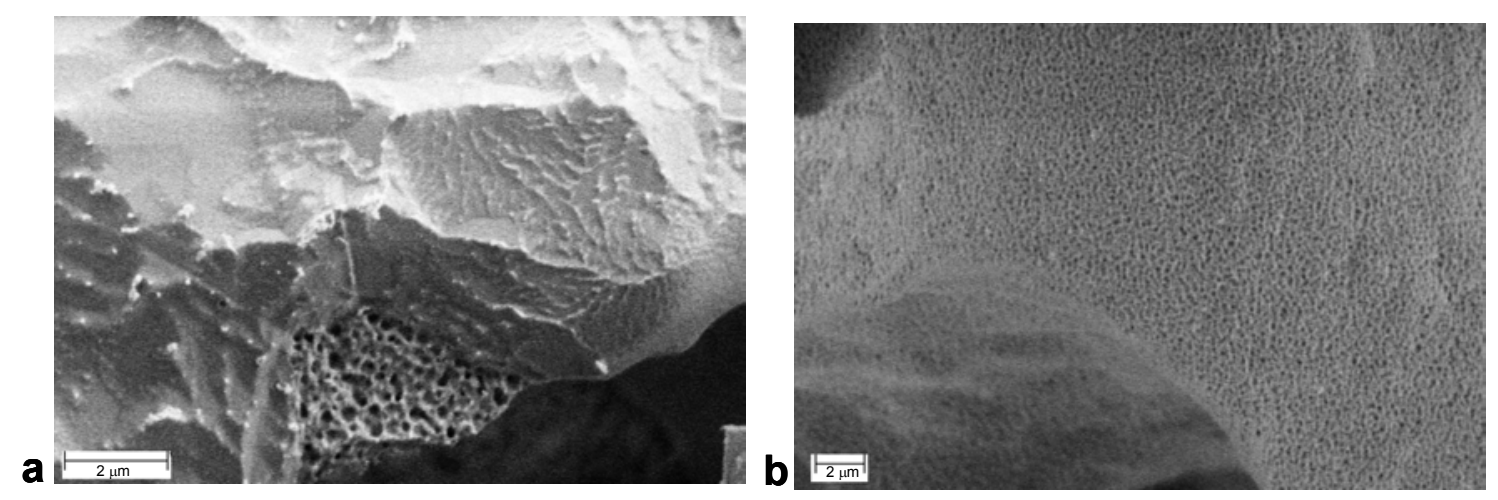

Figure 8. Methane is observed to form gas hydrate microstructure with about ten times bigger pores (a) then ones formed by carbon dioxide (b). The reactions are performed at $-1{ }^{\circ} \mathrm{C}$ and a pressure of $3.5 \mathrm{MPa} \mathrm{CH}_{4}$ (for 23h) and 20MPa $\mathrm{CO}_{2}$ (for 18h), respectively.

Indistinguishable porous microstructures were observed in the FE-SEM images of the gas hydrate samples formed at different thermodynamic conditions from hydrogenated and deuterated systems (Fig. 9). 

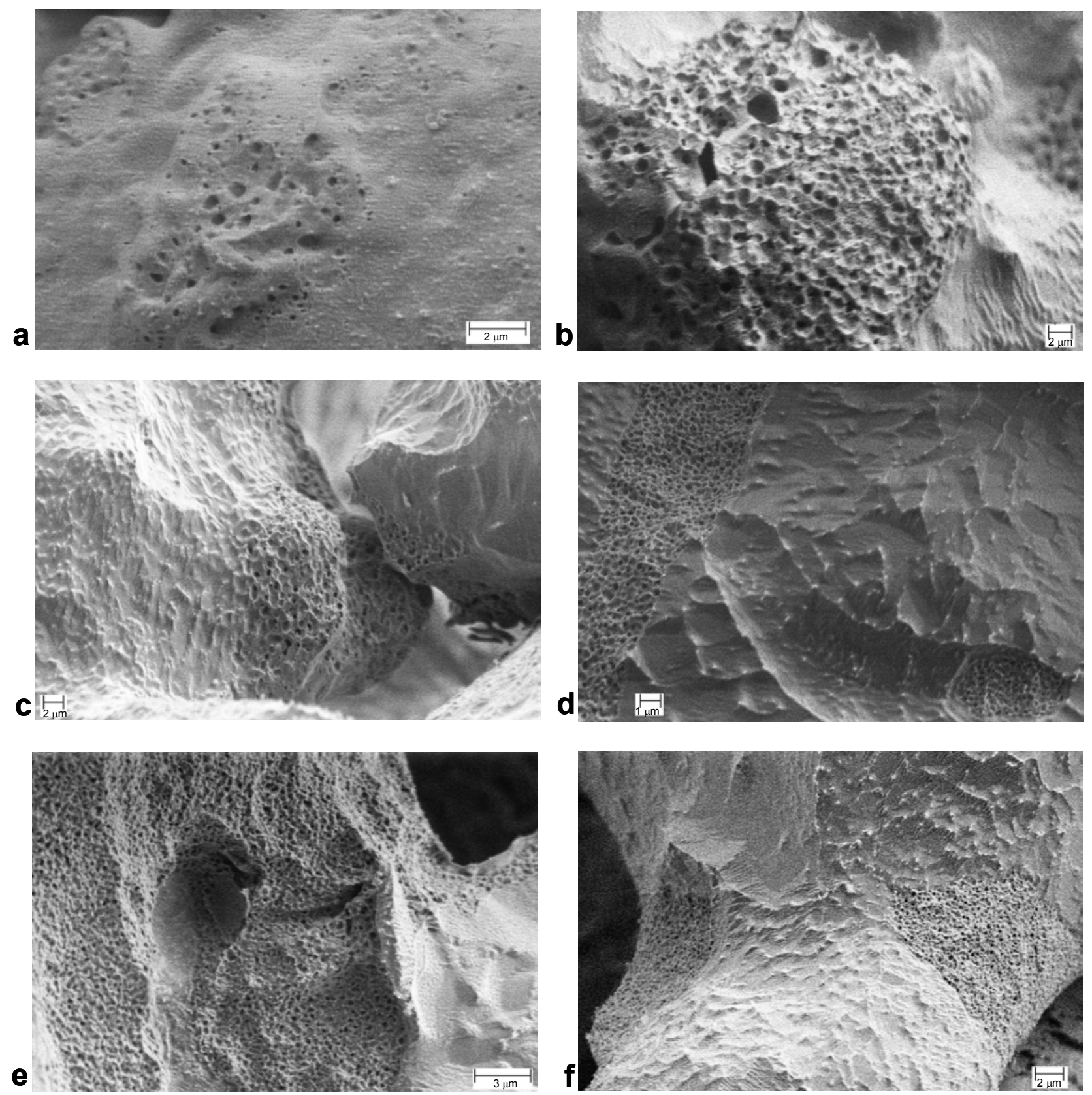

Figure 9. Different samples revealed the unique gas-hydrate porous microstructure. A deuterated $\mathrm{CH}_{4}$-gas hydrate after $11.5 \mathrm{~h}$ of formation at $-43^{\circ} \mathrm{C}$ and $3.5 \mathrm{MPa}$ is shown in (a). Hydrogenated methane clathrate structures grown at $6.0 \mathrm{MPa}$ and $-20^{\circ} \mathrm{C}$ (for 23h) and $-15^{\circ} \mathrm{C}$ (for 14h) are presented in (b) and (c), respectively. The images (d) and (e) show the porous microstructure of $\mathrm{D}_{2} \mathrm{O}-\mathrm{CH}_{4}$ formed at $6.0 \mathrm{MPa}$ and temperatures of $5^{\circ} \mathrm{C}(8 \mathrm{~h})$ and $-1^{\circ} \mathrm{C}(18 \mathrm{~h})$, respectively. A similar structure is observed at a temperature of $-1{ }^{\circ} \mathrm{C}$ and a lower pressure of 3.5 MPa. 
FE-SEM images of a gas hydrate formed for a long period of time are shown in Fig. 10. The reacted sample was consolidated and it had to be broken in order to get a part of it for the SEM investigations. Afterwards, the sample, providing some inner surfaces, showed a hydrate growth in the open pore space in the sample, beyond the initial ice grains.
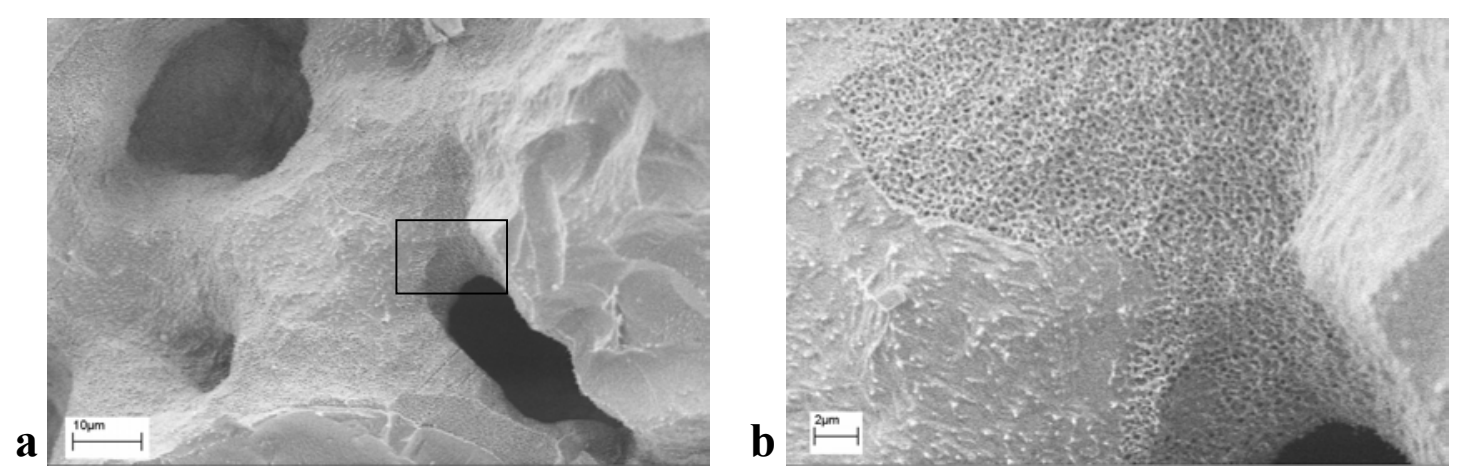

Figure 10. $\mathrm{D}_{2} \mathrm{O}$ ice-grains covered and sintered together by $\mathrm{CH}_{4}$-hydrate after $55 \mathrm{~h}$ of reaction at $6.0 \mathrm{MPa}$ and $268 \mathrm{~K}$. A larger magnification of the left-corner area (b) showed the porous hydrate spreading into the open pore space of the initial ice sample.

The obtained FE-SEM images showed that there is no obvious dependency of the pore size neither on the pressure and temperature nor on the time of reaction. The porous microstructure with pores of a mean size in the order of several hundred nm was observed in all imaged methane clathrate samples.

\subsubsection{Methane Gas Hydrate Growth - FE-SEM approach}

The unique submicron porous structure of the $\mathrm{CH}_{4}$-gas hydrate was used to observe the ice-tohydrate transformation using the FE-SEM technique. A series of "interrupted" runs of a methane clathrate growth at pressure of $6.0 \mathrm{MPa}$ and a temperature of $-10^{\circ} \mathrm{C}$ was performed as follows: eight samples of hydrogenated ice powder were let to react with methane gas in the laboratory set-up used for the pVT kinetic measurements (see Chapter 3.3). The reactions were stopped after different time periods $-30 \mathrm{~min}, 1 \mathrm{~h}, 1.5 \mathrm{~h}, 2 \mathrm{~h}, 3 \mathrm{~h}, 4 \mathrm{~h}, 8 \mathrm{~h}$ and $24 \mathrm{~h}$. The quenched samples were stored in liquid nitrogen and then imaged by FE-SEM. Typical examples of observed different steps of the $\mathrm{CH}_{4}$-hydrate formation were selected from a few hundred electron micrographs and are shown in Fig.11-14. The boxes on the images (on the left) mark areas shown at a 
magnification in the black-bordered pictures (on the right). Overviews of the imaged samples are shown in the small central pictures.

The $\mathrm{CH}_{4}$-hydrate phase was identified with the observed macroporous structure. Its appearance and spreading out on the ice surface was followed by an imaging of samples reacted for different periods. At the beginning of the reaction a formation (nucleation) of hydrate patches on the ice surface and in the cracks of the ice grains was observed. Then the porous microstructure was found to grow simultaneously as a filling of the cracks, spreading on the ice surface and necking between adjacent particles. Subsequent dramatic changes of the grain surface with facetted crystallites growing outward were observed after $4 \mathrm{~h}$ of reaction. A continuing filling of the grain cracks was observed as well. Afterwards the gas hydrate microporous structure filled the voids between the ice spheres causing a sintering of the initial sample and leading to a macroscopically observed sample consolidation. After $24 \mathrm{~h}$ of methane hydrate formation at this temperature the original ice grain structure could not be distinguished any more. 

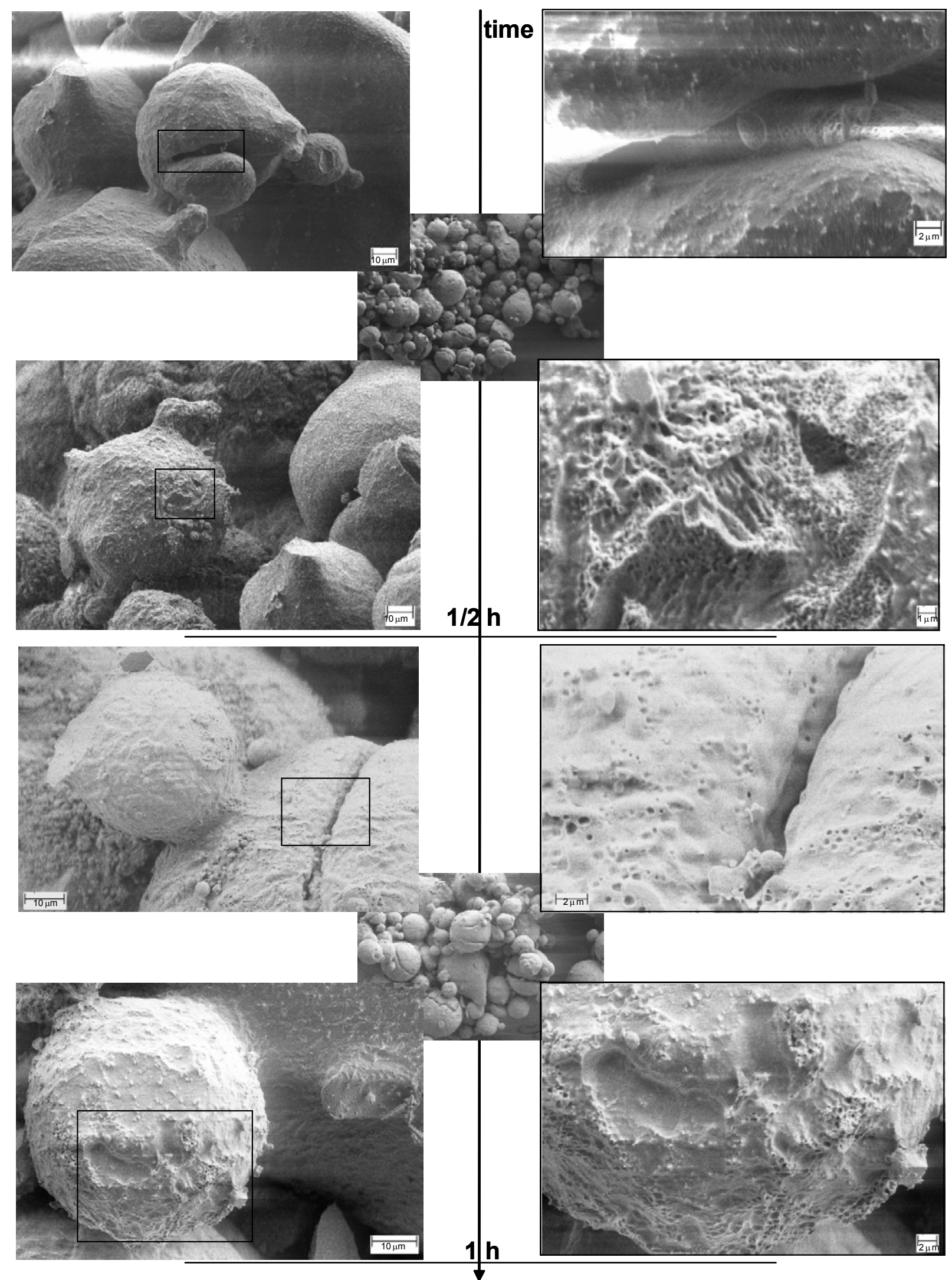

Figure 11. FE-SEM images of $\mathrm{CH}_{4}$-gas hydrates grown at $6.0 \mathrm{Mpa}$ and $-10^{\circ} \mathrm{C}$ during two runs stopped after 30 min and $1 \mathrm{~h}$ of reaction. 


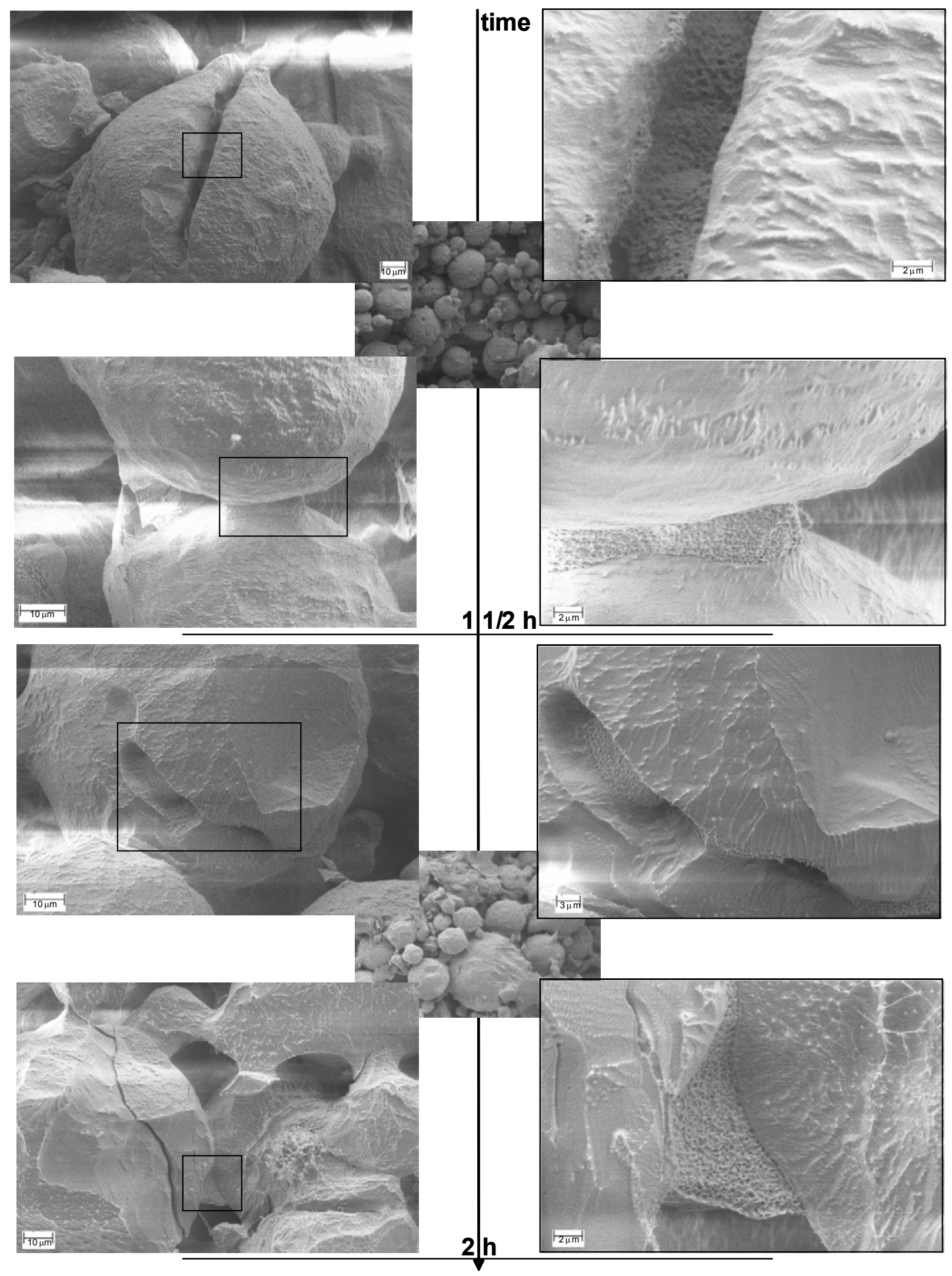

Figure 12. FE-SEM images of $\mathrm{CH}_{4}$-gas hydrates grown at $6.0 \mathrm{Mpa}$ and $-10^{\circ} \mathrm{C}$ during two runs stopped after 90 min and $2 \mathrm{~h}$ of reaction. 


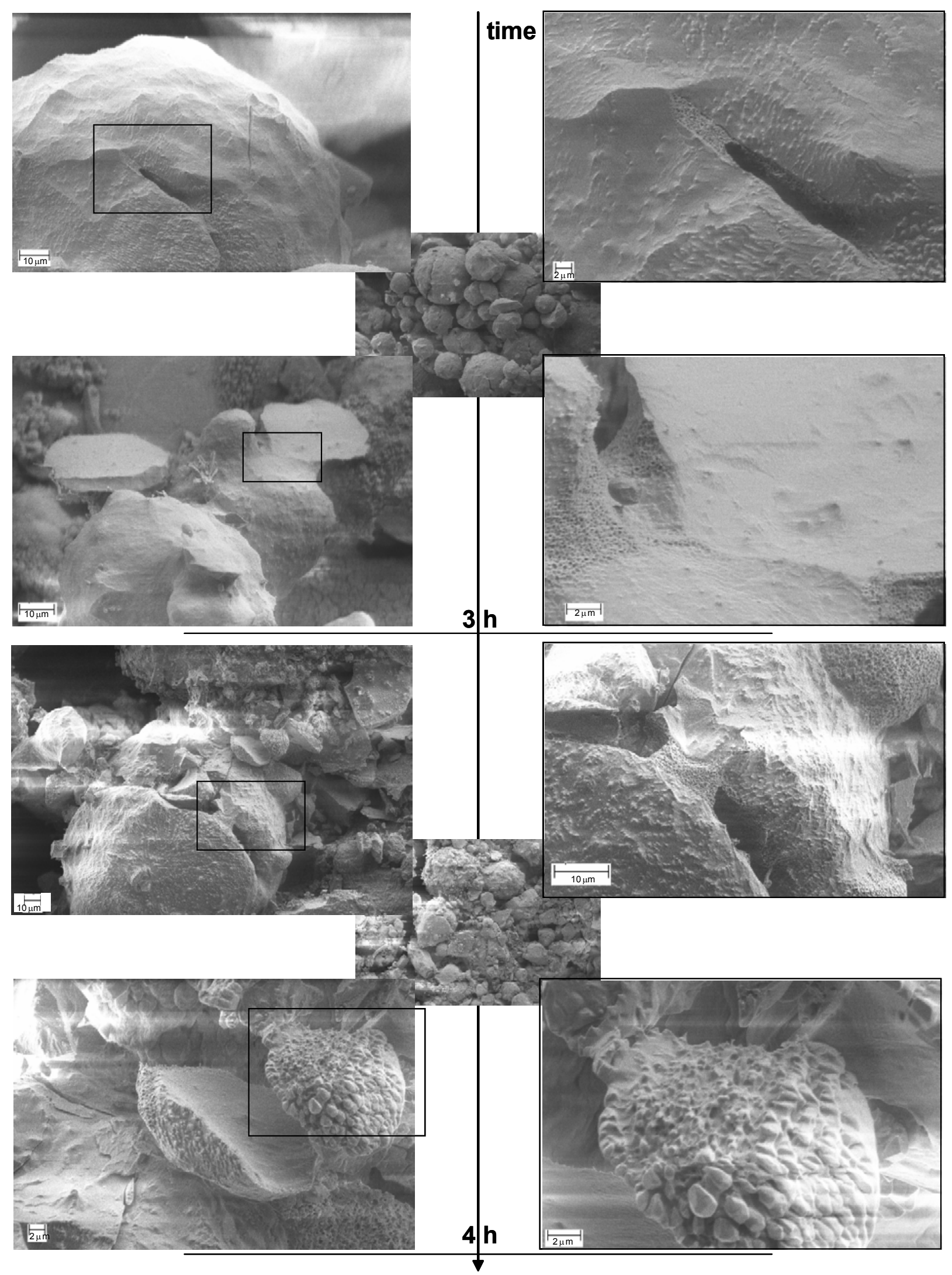

Figure 13. FE-SEM images of $\mathrm{CH}_{4}$-gas hydrates grown at 6.0Mpa and $-10^{\circ} \mathrm{C}$ during two runs stopped after $3 \mathrm{~h}$ and $4 \mathrm{~h}$ of reaction. 

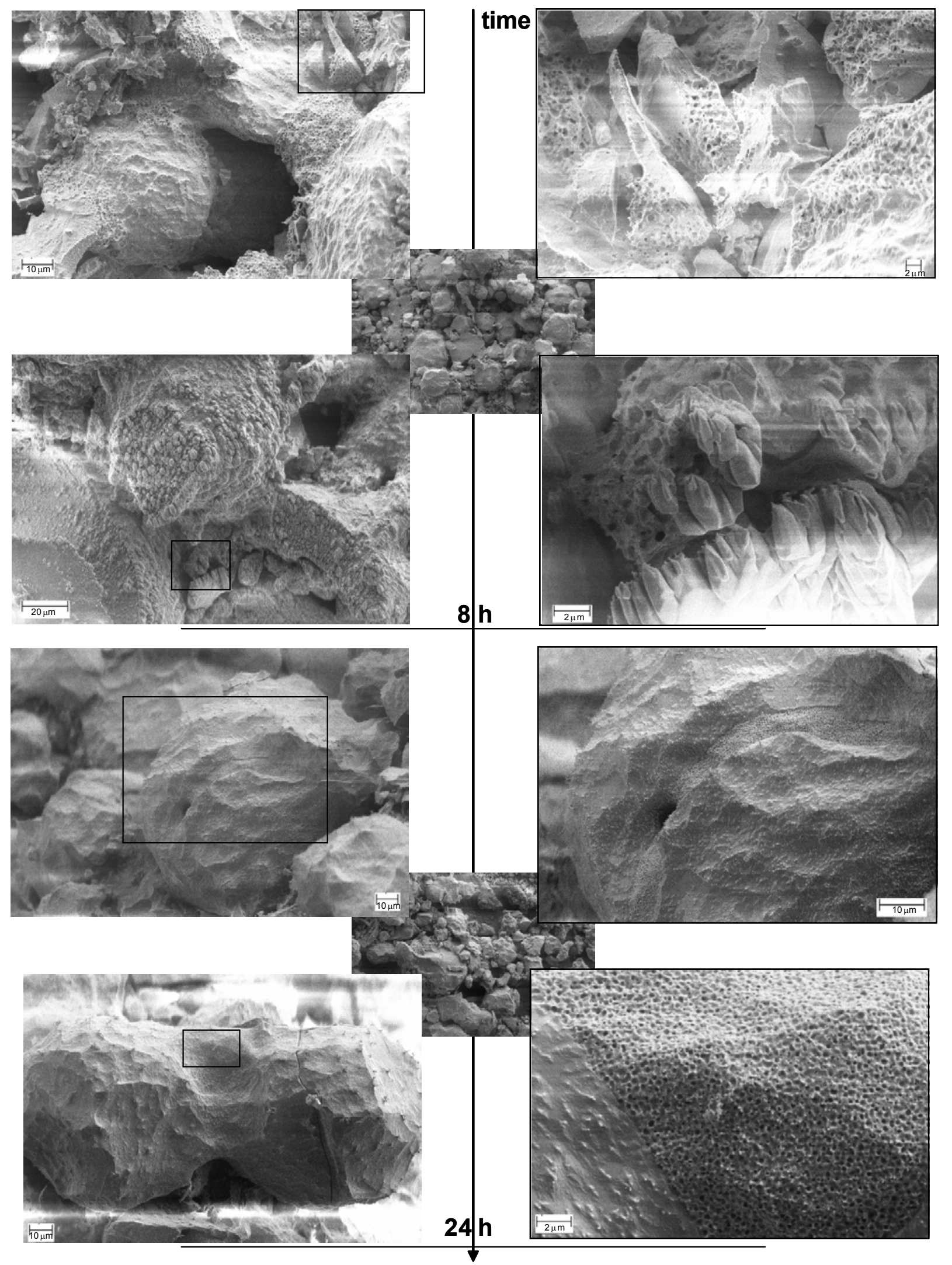

Figure 14. FE-SEM images of $\mathrm{CH}_{4}$-gas hydrates grown at $6.0 \mathrm{Mpa}$ and $-10^{\circ} \mathrm{C}$ during two runs stopped after $8 \mathrm{~h}$ and $24 \mathrm{~h}$ of reaction. 
The collected FE-SEM images of the state of the $\mathrm{CH}_{4}$-hydrate samples formed during the interrupted runs permitted a qualitative picture of the hydrate growth to be made. Figure 15 shows a schematic drawing of the changes of one grain from the initial sample, which transforms during a reaction.

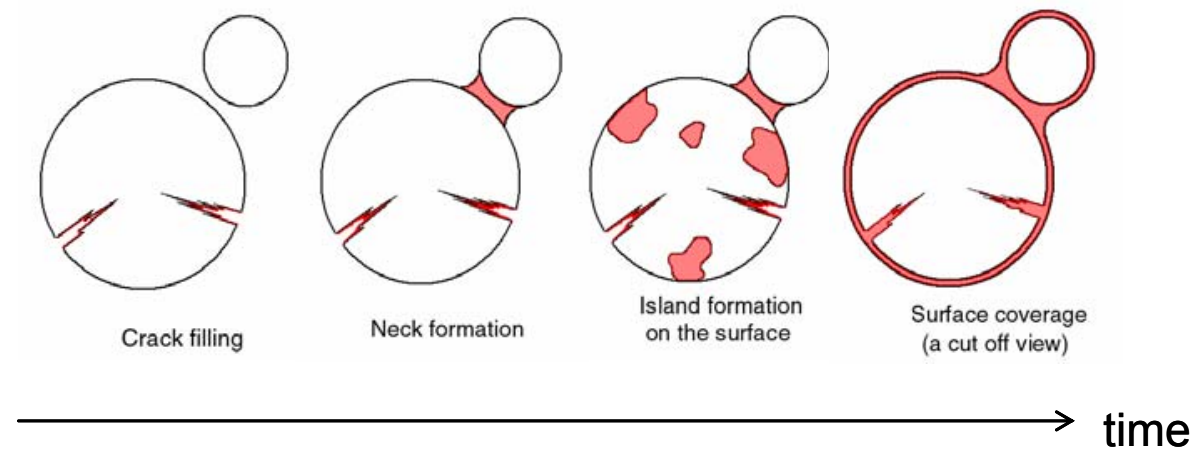

Figure 15. Sketches of different steps of a hydrate formation from ice spheres observed by FE-SEM (see the discussion in the text).

Starting from a well-defined ice powder of spherical grains (see Ch. 3.4.4) the gas-hydrate porous structure was observed to develop initially as a crack filling, necking of the ice grains and formation of patches (islands) on the ice surface (Fig. 11-12). These processes were observed to develop simultaneously at the temperature of the reaction $\left(-10^{\circ} \mathrm{C}\right)$. Subsequently the porous gashydrate structure grew and covered largely the ice surface (Fig.13) leading to a consolidated sample in which, however, the original ice grain structure can still be recognized. The final consolidated state of the sample (Fig. 10,14) was observed as a compact product with irregular shapes of the ice-hydrate particles and a few open pores spaces visible in the FE-SEM after the breaking of the sample. It should be mentioned also that within one sample different grains were observed at different reaction states, i.e. the reaction was developed at some places faster then at others. The FE-SEM observations of methane hydrates at different ages suggested that an ice grain undergoes different stages during its transformation to a hydrate. The clathrate formation starts with an initial coating of the ice surface followed by a spreading inward and outward from the initial ice grain surface. 


\subsection{Summary}

The use of different experimental methods to study the gas hydrate formation kinetics can be summarized as follows. The neutron diffraction method allowed the clathrate fraction to be obtained with a precision of a fraction of a percent even at very short times of data acquisition (30-60 s). In this way, the very initial part of the hydrate formation at temperatures of $230-$ 272K was deduced and no induction period was observed. All reactions started immediately (within a time resolution of few seconds) after applying of gas pressure higher than the decomposition one. Studies of the reaction in hydrogenated systems were also attempted using the neutron diffraction method but the precision of the obtained clathrate fractions was much lower than the one in the deuterated systems. However, the kinetic results from the hydrogenated samples did not indicate (within the large errors) any deviation from the reaction behavior of the deuterated samples. In situ X-ray diffraction studies of the gas hydrate formation were also attempted. A gas consumption (pVT) method was found as an alternative method to study in situ the methane hydrate growth. Although the precision of the obtained hydrate fraction was not as good as the one reached by the neutron diffraction, the gas consumption method was useful to make kinetic studies at different thermodynamic conditions in hydrogenated and deuterated systems. In particular, the pVT method showed a long-term development of the hydrate formation reaction. The use of a FE-SEM technique allowed the microstructure of the reacting samples to be observed. The collected images suggested the existence of different stages of the reaction starting with an initial hydrate coverage of the ice surface, followed by a hydrate spreading in the initial voids between the ice grains. Moreover, the gas hydrate formation was found to proceed with different speeds in the different parts of the sample. Thus, the measured rate of $\mathrm{CH}_{4}$-hydrate formation should be considered as an average quantity for the entire sample. 


\section{References}

Chazallon, B. (1999). Clathrates d'hydrate d'azote, d'oxigène et d'air - Etude par diffraction neutronique et spectroscopie Raman, Dissertation, Universität Göttingen.

Convert, P.; Hansen, T.; Oed, A.; Torregrossa, J. (1998). D20 high flux two axis neutron diffractometer, ICNS'97Proceedings, Physica B 241-243, 195-197.

Convert, P.; Hansen, T.; Torregrossa, J. (1999). The high intensity two axis neutron diffractometer D20 - first results. Proceedings of EPDIC6, Budapest 1998, Materials Science Forum (Trans. Tech. Publications), 321-324, 314-319.

Dianoux, A.; Lander, G. (2002). Neutron data booklet, Institut Laue-Langevin, France.

Hammersley, A. (2003). FIT2D, ESRF, France. http://www.bio.aps.anl.gov/mirror/www.esrf.fr/FIT2D/ftp/fit2d_11_012_i686_W2000.exe

Howard, C. J. (1982). The approximation of asymmetric neutron powder diffraction peaks by sums of Gaussians, J. Appl. Cryst. 15, 615-620.

International thermodynamic tables of the fluid state. [Vol.] 3: Carbon Dioxide (1973). International Union of Pure and Applied Chemistry, Pergamon Press.

International thermodynamic tables of the fluid state. [Vol.] 5: Methane (1976). International Union of Pure and Applied Chemistry, Pergamon Press.

Klapproth, A. Strukturuntersuchungen an Methan- und Kohlenstoffdioxid Clathrat-Hydraten (2002). Dissertation, Universität Göttingen.

Larson, A. C. and Von Dreele, R. B. (1990). GSAS - General Structure Analysis System Report LAUR, 86-748.

Richard, D.; Ferrand, M.; Kearley, G. J. Large Array Manipulation Program (LAMP), Institut Laue-Langevin (ILL), Grenoble, France, ftp://ftp.ill.fr/pub/cs/

Rietveld, H. M. (1967). Line profiles of neutron powder-diffraction peaks for structure refinement. Acta Crystallogr., 22, 151-2. 
Rietveld, H. M. (1969). A profile refinement method for nuclear and magnetic structures. J. Appl. Crystallogr., 2, 65-71.

Sloan E. D., Jr. Clathrate hydrates of natural gases (1998). Second edition, Marcel Dekker Inc.:New York.

Thompson, P.; Cox, D. E.; Hastings, J. B. (1987). Rietveld refinement of Debye Scherrer synchrotron X-ray data from $\mathrm{Al}_{2} \mathrm{O}_{3}$, J. Appl. Cryst. 20, 79-83.

Walker, M.B. General Editor of The Wordsworth Dictionary of Science \& Technology (1995). (C) W\&R Chambers Ltd and Cambridge University Press. 


\section{Chapter 4}

\section{Multistage Model}

A phenomenological approach tries to connect the evolution of a system with macroscopically observable quantities such as a composition, temperature, pressure, volume and time. It expresses the results in terms of "rate constants" and the relevant macroscopic parameters.

\subsection{What is phenomenological modelling?}

The modelling of a certain process is the selection of assumptions, which give the possibility to calculate and predict the properties of the system. One way is to obtain a closed mathematical expression giving a property as a function of selected variables. When the model cannot be expressed in a closed mathematical form results can be obtained numerically by an iterative method.

One possible way to obtain knowledge of how the nature is functioning is to define some hypothesis resulting in a model and test it by a comparing of its predictions with experimental information. It is not of a particular importance whether the predictions are made by an analytical calculation or by some numerical method. 


\subsection{Multistage Model of Gas Hydrate Growth from Ice Powder}

As it was already discussed in Ch.1 the initial gas hydrate formation is fast and then slows down (Fig.1, Ch. 1). Earlier on shrinking-core models formulated for a single ice particle were used to simulate a diffusion-limited clathrate-crystal growth on an ice surface (Takeya et al. (2000), Henning et al. (2000), and Wang et al. (2002)). A porous gas hydrate structure, observed by FESEM (Klapproth, 2002; Klapproth et al., 2003; Kuhs et al., 2000; Suess et al., 2002), led to a multistage model of the clathrate formation from ice (Salamatin and Kuhs, 2002; Kuhs and Salamatin, 2003 a, 2003 b; Staykova et al. 2002, 2003). It associates the initial fast gas-hydrate growth with a coating of the ice surface with a hydrate film. The density of the water in a crystalline hydrate lattice of both types (I and II) is noticeably less than the one in the ice. Thus, the excess water molecules must be partly "evacuated" from the ice-hydrate contact area to vacate additional space for the newly formed hydrate structure. Thus, the pores would provide a transfer of the water and gas molecules until the pores became closed. Then diffusion would control the proceeding of the gas hydrate reaction. The phenomenological multistage model of gas hydrate formation from a well-described ice powder defines an initial stage (I) of a hydrate film spreading over the ice surface and two subsequent stages limited by (II) a clathration reaction at the icehydrate interface and (III) the gas and water transport (diffusion) through the hydrate shells surrounding the shrinking ice cores. As a principal parameter $\alpha$ defining the degree of a reaction the model uses a molar fraction of ice converted to hydrate.

\subsubsection{Basic Shrinking Ice-Core Model}

The multistage model used a geometrical description of a powder's particle growth developed by Arzt (1982) for a random dense packing of monodisperse spheres on the basis of the concept of Voronoi cells associated with the initial powder structure. The build-up of the starting material was characterised by an average number of contacts per particle (coordination number) $Z_{0}$ and a relative slope $C$ of the random packing density function. Experimental estimates for powder packing parameters deduced by Arzt (1982) were consistent with the observed porosity of the ice powder samples. 
During the clathrate formation process ice spheres of initial radius $r_{0}$ were transformed to ice cores of radius $r_{i}$ covered by the growing hydrate layers, which were modelled as spherical shells of external radius $r_{h}$ truncated at the inter-grain contact areas (Fig. 1).

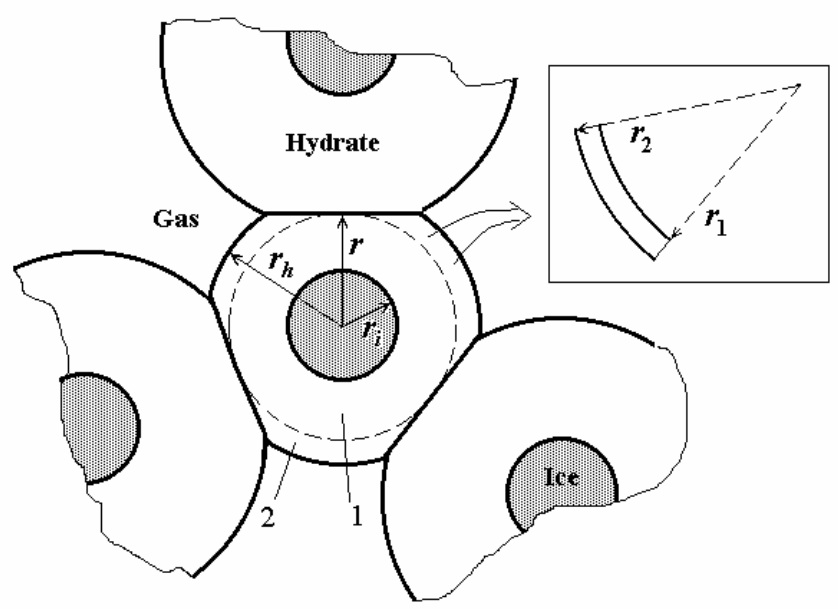

Figure 1. Gas-hydrate expansion into the open pore space of the ice sample shown by a schematic drawing of hydrate shell growth around a shrinking ice core. The dashed-line circle corresponds to the initial ice sphere of radius $r_{0}$. Notations of the convex truncated sub-layer 2 surrounding the inner spherical hydrate layer 1 were made for the discussions in Ch. 4.2.3.

In accordance with Salamatin and Kuhs, 2002 the reduction of the ice-core radius $r_{i}$ due to the hydrate layer growth was described by a mass balance equation

$$
\frac{d r_{i}}{d t}=-\delta_{0} \omega_{S} e^{-\omega_{S} t}-\frac{\omega_{V}}{\rho_{i}}\left(1-e^{-\omega_{S} t}\right)
$$

Here $\delta_{0}$ was the thickness of the ice layer converted to an initial hydrate film of thickness $d_{0}=\delta_{0}(1+E)$ spreading over the ice-grain surface; $\omega_{S}$ and $\omega_{V}$ denoted rates of the ice-surface coating and the ice-to-hydrate transformation, respectively.

The hydrate-phase expansion coefficient $E$ giving a proportion of the hydrate volume excess with respect to the consumed ice volume $E=\frac{\rho_{i}}{\rho_{h w}\left(1-\varepsilon_{h}\right)}-1$, was expressed via the molar density of ice $\rho_{i}$, the molar density of water $\rho_{h w}$ in a hydrate and meso-porosity $\varepsilon_{h}$ of the clathrate phase.

$\omega_{S}$ was defined as the fraction of the open (exposed to the ambient gas) ice surface which became covered by the initial hydrate film during a unit time period, while $\omega_{V}$ was the number of 
ice moles transformed to a hydrate phase per unit of time on a unit area of ice surface after its coating. Depending on the rate-limiting step at each stage of the hydrate formation process, $\omega_{V}$ described either the rate of the clathration reaction or the rate of the gas and water mass transfer through the hydrate shell. The parameter $\delta_{0}$ was small compared to the initial grain size $r_{0}$, whereas the rate of the initial hydrate film formation was assumed to be much higher than the one of the hydrate layer growth after the surface coating.

As a driving force of the hydrate formation was assumed the supersaturation of the gasice-hydrate system, $\ln \left(f / f_{d}\right)$, expressed via fugacities $f$ and $f_{d}$ of the gaseous phase at the imposed and decomposition pressures $P$ and $P_{d}$ at a given temperature $T$. For each stage, this force determined the clathration kinetics and was distributed among different steps of the ice-tohydrate conversion in proportion to their apparent resistances; namely, $k_{S}^{-1}$ for the initial hydrate film spreading over the ice surface, $k_{R}{ }^{-1}$ and $k_{D}{ }^{-1}$ for the clathration reaction and gas/water permeation through the hydrate layer, respectively. Conventionally, the following relations were assumed:

$$
\omega_{S}=k_{S} \ln \frac{f}{f_{d}}, \quad \omega_{V}=\frac{k_{R} k_{D}}{k_{R}+k_{D}} \ln \frac{f}{f_{d}}
$$

The clathration rate constants were assumed to be Arrhenius-type functions of temperature:

$$
k_{J}=k_{J}^{*} \exp \left[\frac{Q_{J}}{R}\left(\frac{1}{T_{*}}-\frac{1}{T}\right)\right], \quad J=S, R, D,
$$

where $k_{J}^{*}$ and $Q_{J}$ denoted the clathration rate constant at the reference temperature $T_{*}$ and the activation energy of the $J$-type step, respectively, and $R$ was the gas constant.

The phenomenological model (1)-(3) was considered as a theoretical basis for the detailed analysis of the hydrate formation kinetics.

\subsubsection{Model Extension Including Crack Filling}

Thermal stresses, which occurred in freezing water droplets sprayed into liquid nitrogen during preparation of ice powder, resulted in fracturing of some of ice spheres (Ch.3.4.4). As it was discussed by Genov at al., 2004, a crack-filling part of the initial surface coverage had to be distinguished as a first step of the clathration reaction at low temperatures. Based on the FE-SEM observations, the crack-filling process, schematically shown in Fig. 2, was assumed as an 
independent relatively short sub-stage of the initial stage I developing simultaneously with the hydrate film patches spreading over the spherical surface of the reference grain.

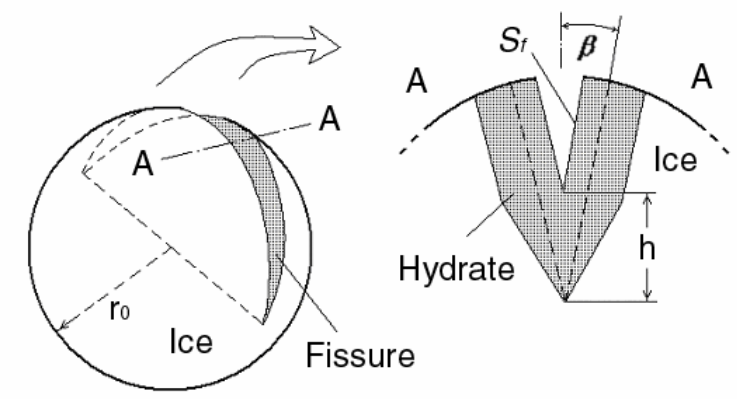

Figure 2. Schematic drawing of the hydrate formation in an ice-grain fissure and its A-A cross-section. The ice at the cleavage sides $S_{f}$ is converted to hydrate at constant rate $\omega_{R}{ }^{\prime}$, and the height $h$ of the fissure filling linearly increases with time. For further notations see text.

The volume filling degree $\chi$ in a reference ice grain of initial radius $r_{i 0}$ was calculated by means of analogues of Eqs. (1) and (2) for hydrate formation on the crack surface, designating all corresponding characteristics by primes (see Appendix D). Thus, for instance, the thickness $\delta_{0}{ }^{\prime}$ of the ice layer converted to the initial hydrate film spreading over the crack sides was introduced together with the respective rates $\omega_{S}{ }^{\prime}$ and $\omega_{R}{ }^{\prime}$ of the crack surface coating and the clathration reaction, the latter two being related to the temperature dependent rate constants $k_{S}^{\prime}$ and $k_{R}^{\prime}$ with activation energies $Q_{S}^{\prime}$ and $Q_{R}^{\prime}$. Then, as explained in Appendix D, for the mean crack-opening angle $\beta$ the average height $h$ of the fissure filling (see Fig. 2) normalised by $r_{i 0}$ was explicitly expressed (at constant $\omega_{R}{ }^{\prime}$ ) versus time $t$ :

$$
\begin{gathered}
\xi=h / r_{0}=a^{\prime}\left(1-e^{-\omega_{s}^{\prime} t}\right)+b^{\prime} t, \quad h<r_{0}, \\
a^{\prime}=\frac{E}{\beta r_{0}}\left(\delta_{0}^{\prime}-\frac{\omega_{R}^{\prime}}{\rho_{i} \omega_{S}^{\prime}}\right), b^{\prime}=\frac{E \omega_{R}^{\prime}}{\beta \rho_{i} r_{0}},
\end{gathered}
$$

until the complete filling is reached at $h=r_{i 0}$, i.e. $\xi=1$.

The area $S_{f}$ of each crack side exposed to the ambient gas decreased as the hydrate filled the fissure. The volume of hydrate growing in the crack was calculated in Appendix E by integration of the incremental mean hydrate layers with respect to $h$, which yields the filling degree $\chi$ in the following form: 


$$
\chi=\frac{3}{4} \pi \xi-\frac{3}{2} \xi \arcsin \xi-\frac{3}{2}\left(1-\xi^{2}\right)^{1 / 2}+\frac{1}{2}\left(1-\xi^{2}\right)^{3 / 2}+1, \quad \xi<1,
$$

and $\chi \equiv 1$ for $\xi>1$.

Although this relationship was derived for the fissure penetrating to the centre of a reference grain, it had a general structure and it can be used for any smaller mean initial relative depth of cracks by appropriate correction of $a^{\prime}$ and $b^{\prime}$ (e.g. by $\beta$ ).

\subsubsection{Analysis of Permeation Resistance of Hydrate Layer}

The shape of the hydrate layer formed from the reference ice grain was represented as a truncated sphere of radius $r_{h}$ (see Fig. 1). The ice core shrank due to the inward growth of the hydrate layer. However, because of the smaller density of water in the porous hydrate phase, the excess water molecules must be transported to the outward hydrate surface exposed to the ambient gas, and the hydrate layer simultaneously expanded into the marco-pore space of the sample between the original ice grains.

The spherical boundary of radius $r$ in Fig. 1 divided the hydrate shell into two sublayers 1 and 2: from $r_{i}$ to $r$ and from $r$ to $r_{h}$, respectively. The permeation (diffusion) resistance of the spherical sub-layer 1 was known from the diffusion theory (Crank, 1975). To estimate the resistance of the truncated sub-layer 2, it was assumed (Staykova et al., 2003) that locally the mass transfer process in it was similar to diffusion through a concave spherical layer of the same thickness with the same total areas of bounding surfaces (see the insert in Fig. 1). The following expression for the permeation rate constant in Eq. (2) could be written:

$$
k_{D}=\frac{\rho_{i} D}{r_{i}} \frac{\sqrt{s} r_{h} r}{\sqrt{s} r_{h}\left(r-r_{i}\right)+r_{i}\left(r_{h}-r\right)}
$$

where $D$ was the apparent gas/water mass transfer (permeation) coefficient (Salamatin et al., 1998). The temperature dependence of the permeation coefficient follows Eq. (3) with ${k_{D}}^{*}$ corresponding to $D^{*}$ at the reference temperature $T *$ in Eq. (6). 


\subsubsection{Polydisperse Variant of Shrinking Ice-Core Model}

The initially used monodisperse approximation of the ice starting material (Staykova et al. (2003)) was quite appropriate to describe the early stages of hydrate formation until the volumetric expansion and geometric interaction of the growing hydrate shells become principal factors controlling the reaction rate. In this case an extension of the model taking into account the difference in the ice grain size and the volume interaction between the particles (valid for the later part of the reaction) was necessary. The geometry of ice powder of randomly packed ice spheres was described in lognormal-sized (polydisperse) approximation, in terms of initial and current mean-volume radii $\left(\bar{r}_{0}\right.$ and $\left.\bar{r}_{i}\right)$ of ice cores. As a starting point of the sample modelling an ice particle of radius $r_{0}$ in the monodisperse powder with random dense packing of ice spheres of average radius $\bar{r}_{0}$, was considered (see Fig. 3 ).

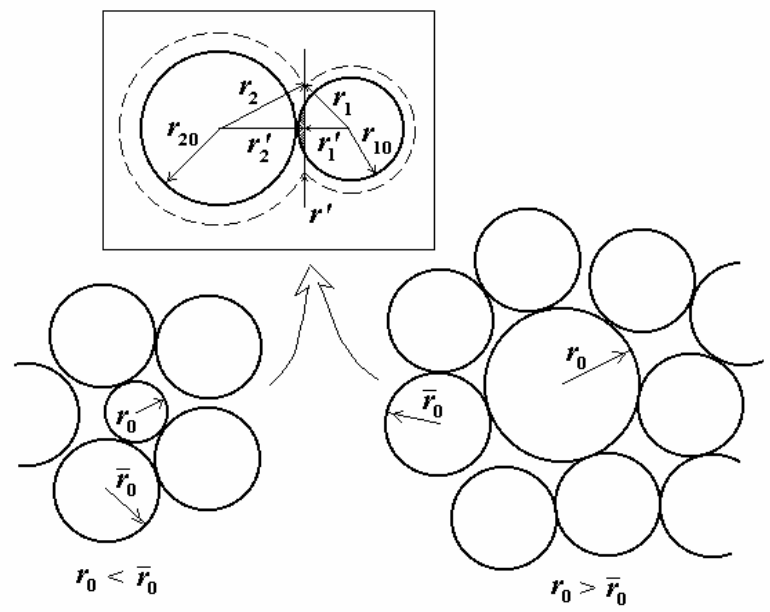

Figure 3. A schematic drawing of the conditionally averaged initial structure of an ice powder sample around a reference particle, which is smaller (left) or larger (right) than the average particle. The insert shows the growth geometry of two spheres in contact.

Arzt's approach was extended to polydisperse powder (Kuhs and Salamatin, 2003 a), when the above scenario was also assumed for the average* ice particles surrounding the reference one. All

\footnotetext{
* Each ice particle in a polydisperse powder with random dense packing had a specific relative environment of surrounding grains and the initial particle size did not determine uniquely the process of its conversion to hydrate. Consequently, the interaction of a reference ice sphere in the powder with neighbouring spheres was described on average. In this context, the above-introduced radii $r_{i}$ and $r_{h}$ should be understood as the mean characteristics of all ice-hydrate particles which developed from initial ice grains of the same radius $r_{0}$. As a result the number of icehydrate particles in each initial size fraction can be considered as constant and the sample at any moment $t$ was modelled by ice-core size distribution function (eq. (E.3), Appendix E).
} 
of them consisted of the inner ice core of radius $\bar{r}_{i}$ surrounded by the truncated spherical hydrate shell of external radius $\bar{r}_{h}$. The existing contact areas between the reference particle and the neighbouring ones increased, and additional contacts were formed as $r_{h}$ and $\bar{r}_{h}$ were growing. The evolution of a single reference ice grain of initial radius $r_{0}$ was modelled in interaction with the simultaneous ice-to-hydrate conversion in the surrounding average monodisperse medium of randomly packed spheres of radius $\bar{r}_{0}$ (see Fig.3 and Appendix E). Correspondingly, the fraction $s$ of the free hydrate surface area exposed to the ambient gas, the specific surface area of macrovoids $S_{m}$ and the macro-porosity $\varepsilon_{m}$ of the sample decreased. Finally, the current sample geometry was related to the ice core radius by means of the hydrate volume expansion factor $E$. Due to the gas-hydrate expansion, the permeability of the pore channels formed by the original ice grains in the sample decreased, and the pores got closed at a certain macro-porosity $\varepsilon_{m c}$, related to a definite value of the free surface fraction $\bar{s}_{c}$ of average particles. This cut off the ambient gas flow towards the particles and stopped the clathration process. In polydisperse powder the closeoff porosity was preferentially reached near the bigger grains, which became isolated with time before their complete transformation to the hydrate phase. All important geometrical characteristics of the polydisperse powder which were necessary to describe the problem (1)-(3) of the conversion of a single ice grain to hydrate in the powder medium and to model the evolution of the ice grain ensemble are given in Appendix F.

First, in accordance with Staykova et al. (2003), the ice-to-hydrate conversion at the earlier stages was assumed as limited by the reaction. Consequently, $\omega_{V}$ is constant $\left(\omega_{V} \approx \omega_{R}\right)$, and the right hand side of Eq. (1) does not depend on $r_{0}$, that is $d r_{i} / d r_{0} \approx 1$ in Eq. (E.3), Appendix E. As a result, the ice-core size distribution function retains the lognormal shape and is simply shifted with time to the left along the $r_{i}$-axis. So, multiplication of Eq. (E.3), Appendix E, by $r_{i}^{2} f_{0}\left(r_{0}\right)$ and its integration with respect to $r_{0}$ yields the averaged equation of the same structure as the one used in the monodisperse approximation (Staykova et al., 2003):

$$
\frac{d \alpha}{d t}=S_{i}\left[\rho_{i} \delta_{0} \omega_{S} e^{-\omega_{S} t}+\bar{\omega}_{V}\left(1-e^{-\omega_{S} t}\right)\right]
$$


where $\alpha$ and $S_{i}$ are determined by Eqs. (E.6), Appendix E, and the mean reaction rate $\bar{\omega}_{V} \approx \omega_{R}$. The asymptotic solution of (1) (Kuhs and Salamatin, 2003 a) in case of a polydisperse system was:

$$
\bar{r}_{i} / r_{0}=1-A\left(1-e^{-\omega_{S} t}\right)-B t
$$

with the mean-volume ice-grain radius $\bar{r}_{0}$ and

$$
A=\frac{1}{\bar{r}_{0}\left(1+\bar{\sigma}_{0}^{2}\right)}\left(\delta_{0}-\frac{\omega_{R}}{\rho_{i} \omega_{S}}\right), B=\frac{\omega_{R}}{\bar{r}_{0} \rho_{i}\left(1+\bar{\sigma}_{0}^{2}\right)},
$$

where $\bar{\sigma}_{0}$ is the relative standard deviation of the initial grain size $r_{0}$ in the ice powder.

Equation (8) required that the plot of $\bar{r}_{i} / \bar{r}_{0}=(1-\alpha)^{1 / 3}$ against time $t$ in the beginning of the hydrate formation during stage II limited by the clathration reaction for $t>>\omega_{S}^{-1}$ should be a straight line with slope $B$ and intercept $1-A$, i.e. $\bar{r}_{i} / \bar{r}_{0}=1-A-B t$

In the case of crack filling, for the reaction-limited kinetics of the hydrate formation process valid for small $\alpha$ the asymptotic relation was:

$$
(1-\alpha)^{1 / 3} \approx 1-\frac{\varepsilon_{f}}{3 E} \chi(\xi)-A\left(1-e^{-\omega_{S} t}\right)-B t
$$

As before, Eq. (9) required that the plot of $(1-\alpha)^{1 / 3}$ against time $t$ in the beginning of the hydrate formation during stage II limited by the clathration reaction for $t>>\omega_{S}^{-1}$ should be a straight line with slope $B$ and intercept $1-A-\varepsilon_{f}(3 E)$.

For the diffusion-limited stage it results from Eq. (6) that the rate of the hydrate formation $\omega_{V}$ in Eqs. (1) and (2) was inversely proportional to $r_{i}$. The averaging procedure applied to the basic Eq.(1) after multiplication by $r_{i}^{2}$ would lead to a term $\left\langle r_{i}^{2} \omega_{V}\right\rangle \sim\left\langle r_{i}\right\rangle$, where the average \langle\rangle was defined in Eq. (E.5), Appendix E. With the assumption of the ice-core size distribution being approximately a lognormal one, Eq. (7) could be written with $\bar{\omega}_{V}$ expressed as

$$
\begin{gathered}
\bar{\omega}_{V}=\omega_{R}\left(1-\frac{1}{1+F \theta}\right), \\
F=\frac{D \rho_{i}}{\bar{r}_{0} k_{R}}, \quad \theta=\frac{\sqrt{s} \bar{R}_{h} \bar{R}}{\bar{R}_{i}\left[\sqrt{s} \bar{R}_{h}\left(\bar{R}-\bar{R}_{i}\right)+\bar{R}_{i}\left(\bar{R}_{h}-\bar{R}\right)\right],}
\end{gathered}
$$


where $\bar{R}_{i}=\bar{r}_{i} / \bar{r}_{0}, \bar{R}_{h}=\bar{r}_{h} / \bar{r}_{0}$, and $\bar{R}=\bar{r} / \bar{r}_{0}$ were the mean normalised radii with $\bar{r}$ determined as average distance from the ice core centre to an average contact plane (see Fig. 1)

$$
\bar{r}=\bar{r}_{h}\left[1-\frac{2(1-\bar{s})}{\bar{Z}}\right], \quad \bar{Z}=\bar{Z}_{0}+C\left(\bar{R}_{h}-1\right) .
$$

The dimensionless complex $F$ in Eq. (10) was a principal parameter responsible for the onset of stage III controlled (or influenced) by the gas/water mass transfer through hydrate shells surrounding the shrinking ice cores. The normalised factor $\theta$ changed from infinitely large values in the beginning of the clathration reaction to the first order of magnitude in the later phase of the gas-hydrate formation. Thus, the ice-hydrate system could never pass to the stage III at large $F$, and $\omega_{V} \approx \omega_{R}$ in Eqs. (2) and (10). For $F<<1$ the stage II would become extremely short and would finish together with the stage I directly replaced by the diffusion-limited stage III. Intermediate values of $F \sim 1$ would correspond to the onset of the stage III simultaneously controlled by both (reaction and diffusion) steps.

More details on the mathematics describing the evolution of the ice grain ensemble in the reacting polydisperse sample could be found in (Kuhs and Salamatin, 2003 a).

Polydisperse model simulations with and without the crack filling parameterisation (Kuhs and Salamatin, 2003 b and 2003 a, respectively) were performed by software POWDER 2 and POWDER 3, respectively (see Appendix G). The average model (Kuhs and Salamatin, 2003 a, 2003 b) is valid until the smaller-size fraction of ice cores would start to disappear while the bigger ice particles would become isolated and would be excluded from the reaction. This would completely change the size distribution in the ensemble of ice cores involved in the ice-to-hydrate transformation process, and the simplified averaged model (7)-(10) breaks down in this case.

\subsection{Conclusion}

A general phenomenological model describes a multistage process of gas hydrate formation from polydisperse ice powder. It assumed that the rate of ice-to-hydrate transformation at the different stages of the process depends linearly on the driving force, i.e. on the supersaturation of the gasice-hydrate system. The model describes three predictable stages. They are the initial stage I of the hydrate-film spreading over the ice surface and the two subsequent stages II and III of the hydrate-layer growth limited by the clathration reaction (including the gas transport along the ice- 
hydrate interface) and by the diffusive gas/water mass transfer through the hydrate shells, respectively. In general the mathematics of the model is quite universal, and allows for different physical interpretations. Therefore it should be considered as a universal multistage model of the hydrate growth. 


\section{References}

Arzt, E. (1982). The influence of increasing particle coordination on the densification of spherical powders. Acta Metallurgica, 30, 1883-1890.

Crank, J. (1975). The mathematics of diffusion. Clarendon Press, Oxford.

Genov, G.; Kuhs, W. F.; Staykova, D. K.; Goreshnik, E.; Salamatin, A. N. (2004). Experimental studies on the formation of porous gas hydrates. American Mineralogist, Vol. 89, No. 8-9, $1228-1239$.

Henning, R.W.; Schultz, A.J.; Thien, Vu; Halpern, Y. (2000). Neutron diffraction sudies of $\mathrm{CO}_{2}$ clathrate hydrate: formation from deuterated ice. Journal of Physical Chemistry A, 104, 5066-5071.

Klapproth, A. (2002). Strukturuntersuchungen an Methan- und Kohlenstoffdioxid-ClathratHydraten. Dissertation, Universität Göttingen.

Klapproth, A.; Goreshnik, E.; Staykova, D.; Klein H.; Kuhs W.F. (2003) Structural Studies of Gas Hydrates. Canadian Journal of Physics, 81, 503-518.

Kuhs W.F.; Klapproth, A.; Gotthardt, F.; Techmer, K.; Heinrichs, T. (2000). The formation of meso- and macroporous gas hydrates. Geophysical Research Letters 27(18), 2929-2932.

Kuhs, W.F.; Salamatin, A.N. (2003 a). Formation of porous gas hydrates from polydispersed powders: Model concepts. Project "Modelling gas-hydrate formation on ice and transport properties of porous hydrates" subject to the BMBF Project "Gas-Hydrate im Geosystem", Res. Rep. No4 (January, 2003).

Kuhs, W.F.; Salamatin, A.N. (2003 b). Modelling formation of porous gas hydrates from polydispersed ice powders, taking account of crack filling. Project "Modelling gas-hydrate formation on ice and transport properties of porous hydrates" subject to the BMBF Project "Gas-Hydrate im Geosystem", Res. Rep. No5 (August, 2003).

Salamatin, A.N.; Hondoh, T.; Uchida, T.; Lipenkov, V.Y. (1998). Post-nucleation conversion of an air bubble to clathrate air-hydrate crystal in ice. Journal of Crystal Growth, 193, 197-218. 
Salamatin, A.N.; Kuhs W.F. (2002). Formation of porous gas hydrates. Proceedings of the Fourth International Conference on Gas Hydrates, Yokohama, May 19-23, 766-770.

Staykova, D.K.; Kuhs, W.F.; Salamatin A.N.; Hansen, T. (2003). Formation of porous gas hydrates from ice powders: diffraction experiments and multistage model. J. Phys. Chem. B, 107, 10299-10311.

Suess, E.; Bohrmann, G.; Rickert, D.; Kuhs, W.F.; Torres, M.E.; Trehu, A.; Linke, P. (2002). Properties and fabric of near-surface methane hydrates at Hydrate Ridge, Cascadia Margin. Proc. 4th Int. Conf. Gas Hydrates, 740-744.

Takeya, S.; Hondoh, T.; Uchida, T. (2000). In-situ observations of $\mathrm{CO}_{2}$ hydrate by X-ray diffraction. Annals of the New York Academy of Sciences, 912, 973-982.

Wang, X.; Schultz, A.J.; Halpern, Y. (2002). Kinetics of ice particle conversion to methane hydrate. Proc. 4th Int. Conf. Gas Hydrates, 455-460. 


\section{Chapter 5}

\section{Interpretation of $\mathrm{CH}_{4}$-Gas Hydrate Kinetic Data}

\subsection{Methane Clathrate Formation - Data and Results}

The experimental neutron-diffraction and gas-consumption methods (Ch. 3.1, 3.3) produced in situ kinetic data on the gas hydrate formation from ice below the quadruple point. Their comparison and analysis is given in the following sections.

\subsubsection{Neutron Diffraction Kinetic Data - Short-Term Growth}

A dependence of the hydrate growth rate on the thermodynamic conditions, the grain size of the starting material and the type of the hydrate former was investigated by neutron diffraction experiments (Ch.3.1).

The Rietveld analyses of sequential powder diffractograms determined the values of gas hydrate phase fraction (or the degree of a reaction - alpha) with an accuracy of about $0.2 \%$. The plots of the clathrate weight fraction versus time were compared for different kinetic runs and analysed (see Fig. 1-6). Each plotted symbol corresponds to a value of the gas hydrate weight fraction obtained from one data file.

A pressure influence on the growth kinetics is illustrated by runs performed at $6.0 \mathrm{MPa}$ and $3.5 \mathrm{Mpa}$, and temperatures of $272 \mathrm{~K}$ and $230 \mathrm{~K}$, i.e. $4.7 \mathrm{~K}$ and $43 \mathrm{~K}$ below the $\mathrm{D}_{2} \mathrm{O}$-ice melting point, respectively (Figure 1). 


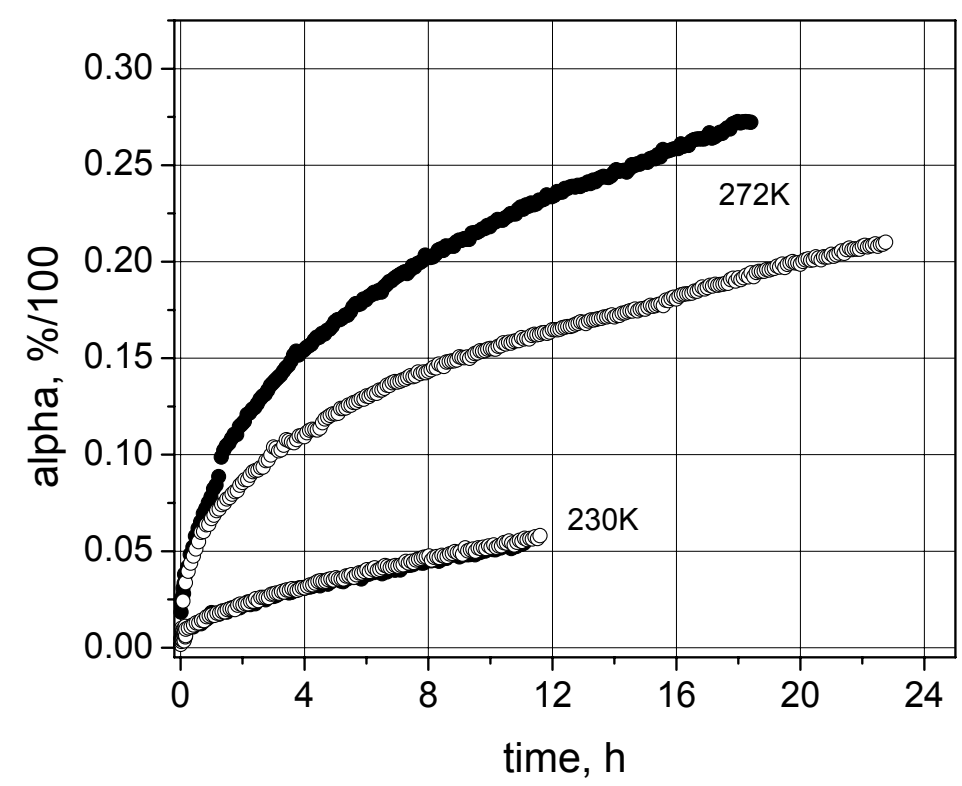

Figure 1. Comparisons of the rates of $\mathrm{CH}_{4}-\mathrm{D}_{2} \mathrm{O}$ hydrate growth at temperatures of $272 \mathrm{~K}(3.5$ and $6.0 \mathrm{MPa})$ and $230 \mathrm{~K}(3.5$ and 6.0 MPa). White and black circles correspond to 3.5 and 6.0 MPa, respectively.

At higher temperature the difference in the pressure clearly influenced the rate of $\mathrm{CH}_{4}$-hydrate formation. An increase of the growth rate with an increase of the pressure from $3.5 \mathrm{MPa}$ to 6.0 $\mathrm{MPa}$ at temperature of $272 \mathrm{~K}$ (i.e. an excess fugacity from 0.36 to 1.2 , respectively) was obtained at three times higher excess fugacity. At temperature of $230 \mathrm{~K}$ the same pressure difference had a minor role on the growth kinetics at an excess fugacity of 4.21 and 6.97 , respectively.

The ice-to-hydrate conversion observed in $\mathrm{D}_{2} \mathrm{O}-\mathrm{CH}_{4}$ systems was definitely a temperature-dependent process (Fig. 2). The observed methane hydrate kinetics revealed an increase of the reaction rate with an increase of the temperature. For instance, about $5 \%$ hydrate was formed for $10 \mathrm{~h}, 1.5 \mathrm{~h}, 20 \mathrm{~min}$ and $10 \mathrm{~min}$ at 230, 263, 270 and $272 \mathrm{~K}$, respectively The reaction at $268 \mathrm{~K}$ developed faster then it was expected for a reaction within the range of 263 and $270 \mathrm{~K}$. 


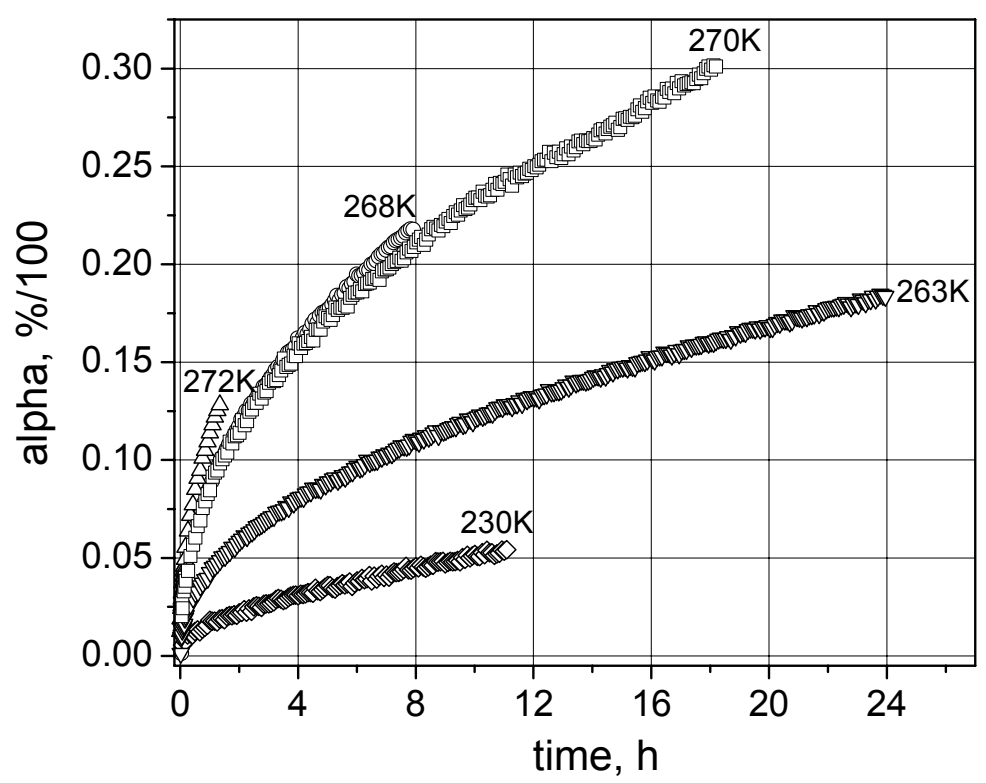

Figure 2. Temperature dependence of the kinetics of $\mathrm{CH}_{4}$-hydrate formation at gas pressure of $6.0 \mathrm{MPa}$.

One short reaction ( 1.5 h) at $272 \mathrm{~K}$ and 6.0 MPa (Fig. 3) was additionally conducted to compare the experimental results obtained with a different mean size of the initial ice powder.

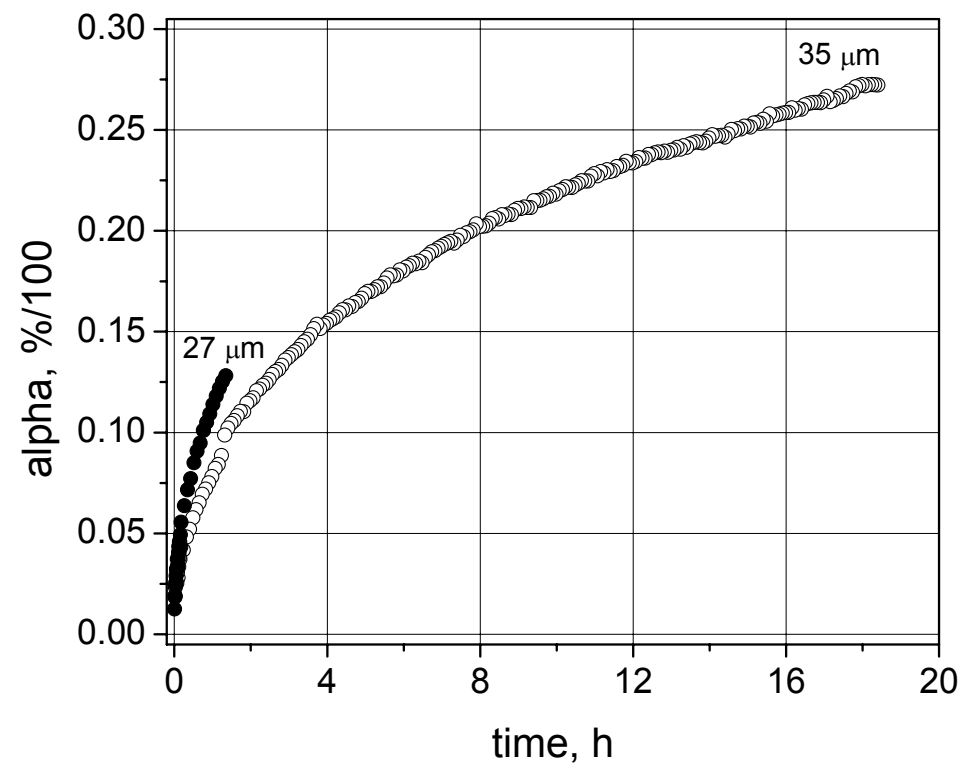

Figure 3. Kinetic curves obtained for the $\mathrm{CH}_{4}-\mathrm{D}_{2} \mathrm{O}$ hydrate formation at identical thermodynamic conditions (6 MPa and $272 \mathrm{~K}$ ) of two experiments with different mean diameters of the ice particles: 70 and $54 \mu \mathrm{m}$ for long and short run, respectively. The reaction rate was inversely proportional to the grain size. 
The reaction rate was noticeably higher in the sample with smaller grains. From these experiments an average factor of $1.3 \pm 0.16$ was determined as a ratio of the degrees of the reactions at a given time. At a constant packing density the number of the ice spheres, placed in a unit volume, would increase with a decrease of their radius. Consequently the reacting ice surface would increase and would give a higher reaction rate.

Data of $\mathrm{CH}_{4-}$ and $\mathrm{CO}_{2}$ - hydrate formations at temperature of $272 \mathrm{~K}$ were compared, showing a clear difference in the reaction kinetics of both gases (Fig. 4).

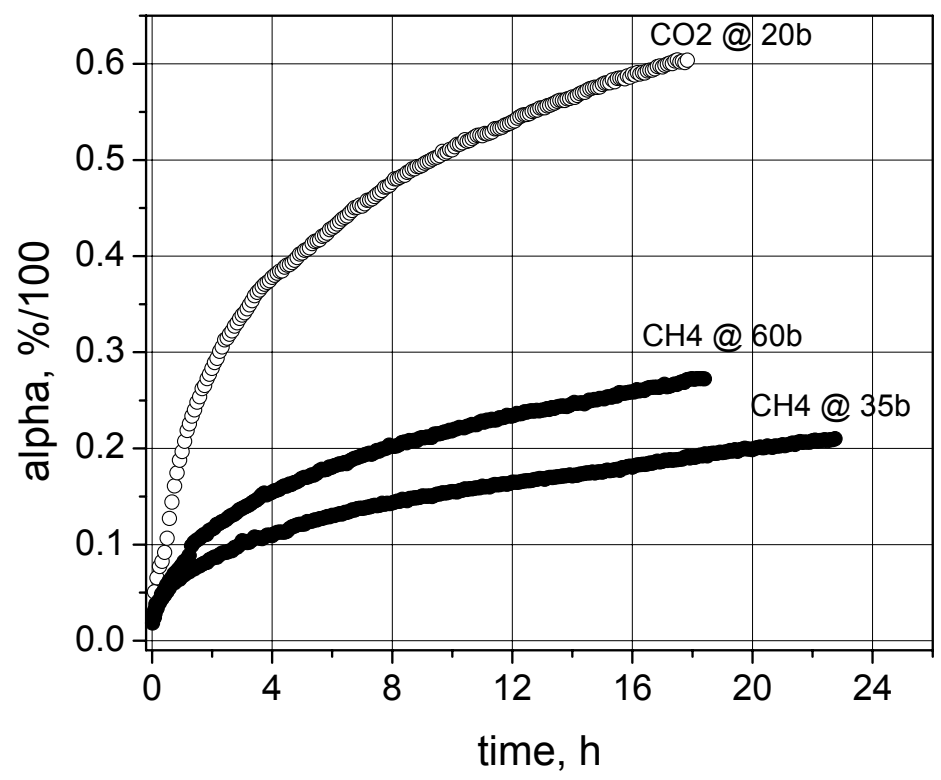

Figure 4. Comparison of hydrate formation rates at two different hydrate formers: $\mathrm{CH}_{4}(3.5$ and 6.0MPa) and $\mathrm{CO}_{2}(2.0 \mathrm{MPa})$ at temperature of $272 \mathrm{~K}$.

The maximum conversion of ice into type I gas hydrate at the end of the runs was $21 \%(27 \%)$ for $\mathrm{CH}_{4}$ and $60 \%$ for $\mathrm{CO}_{2}$ at respective excess fugacities of 0.36 (1.2) and 0.73 . Thus, $\mathrm{CO}_{2}$ reacted about 3 times faster than $\mathrm{CH}_{4}$ at high temperatures.

In case of $\mathrm{CO}_{2}$-clathrate growth, similar to the earlier observations by Fleyfel and Devlin (1991) for a temperature of $150 \mathrm{~K}$, a transient formation of type II structure was observed at 272K (see Fig. 5). 

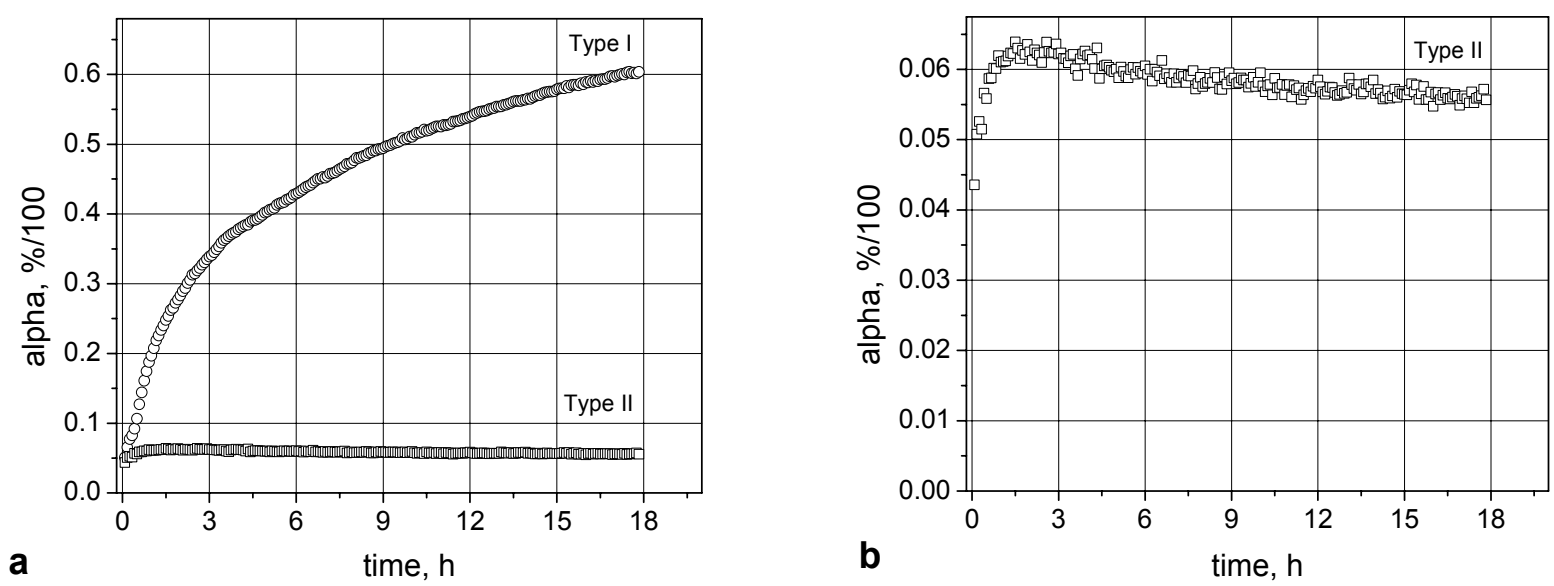

Figure 5. Kinetics of a simultaneous formation of type I and type II structures of $\mathrm{CO}_{2}$-hydrate at $272 \mathrm{~K}$ (a). The development of structure II is shown in more details on a plot (b).

The kinetics of type II $\mathrm{CO}_{2}$-hydrate showed that the structure behaved as a metastable phase, which was formed fast initially and then slowly started to diminish. This pointed to an important detail of the nucleation mechanism that it appears easier to nucleate the thermodynamically less stable type II structure which posses a higher proportion of small cages versus large cages (2:1 for type II and 1:3 for type I hydrates). This observation appeared to be in agreement with the results of ab initio calculations (Chihaia et al., 2004) indicating a higher stability of the small cage buckyball water clusters (see Ch. 2.5).

Intermittent runs performed in the way described in (Ch.3.1.6) provided long-term reactions of $\mathrm{CH}_{4}$-hydrate formation at temperatures of $263 \mathrm{~K}$ and $270 \mathrm{~K}$. Their comparison is shown in Fig. 6. The obtained data gave direct information on a 5-to-6-days regime of the hydrate growth at two different temperatures. The long-term studies of the methane clathrate growth were continued using the gas-consumption method. 


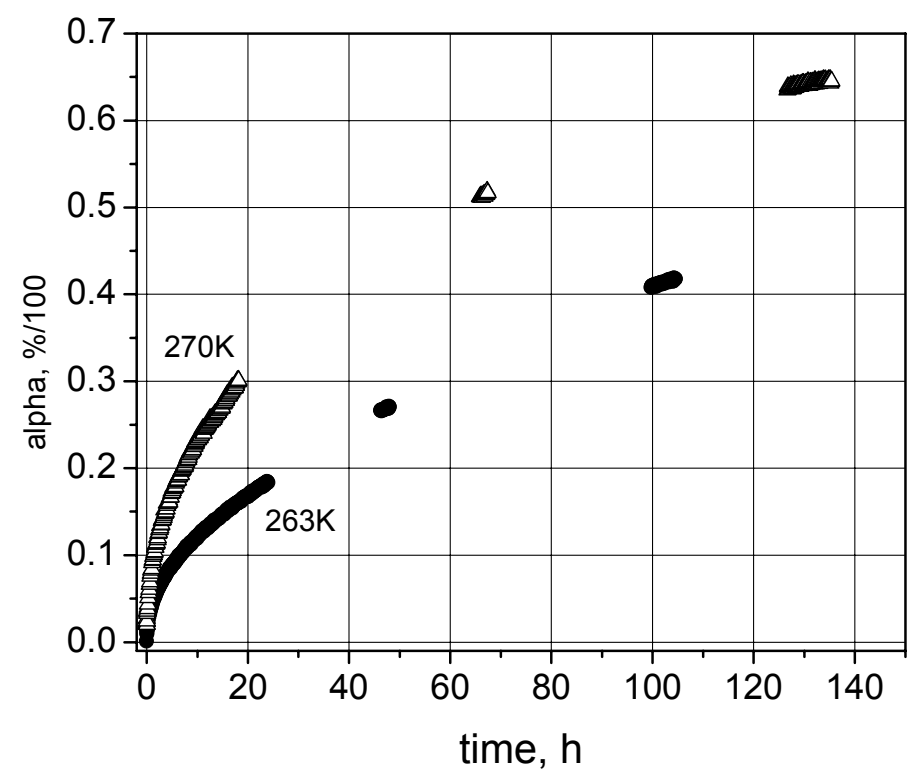

Figure 6. Comparison of the results obtained by long-term runs of $\mathrm{D}_{2} \mathrm{O}-\mathrm{CH} \mathrm{H}_{4}$ hydrate formation at two temperatures: $-3^{\circ} \mathrm{C}$ and $-10^{\circ} \mathrm{C}$, marked with white triangles and black circles, respectively.

\subsubsection{Gas-Consumption Kinetic Data - Long-Term Growth}

The gas-consumption method (Ch.3.3) was used to study the long-term $\mathrm{CH}_{4}$-gas hydrate growth in hydrogenated and deuterated systems at temperatures higher than $245 \mathrm{~K}$. The obtained kinetic data, giving the relative amount of hydrate with an accuracy of about $3 \%$, together with in situ diffraction data (Ch.5.1.1), resulted in an large set of runs studying the temperature, pressure and isotope dependence of the growth rate of methane clathrate.

A dependence of the methane hydrate rate of formation on the temperature for a longterm reaction period (about two weeks) was obtained by a comparison of runs performed with $\mathrm{H}_{2} \mathrm{O}$ - and $\mathrm{D}_{2} \mathrm{O}$ - ice at constant pressure of $6.0 \mathrm{MPa}$ and temperatures of 245-268K (see Fig.7). 


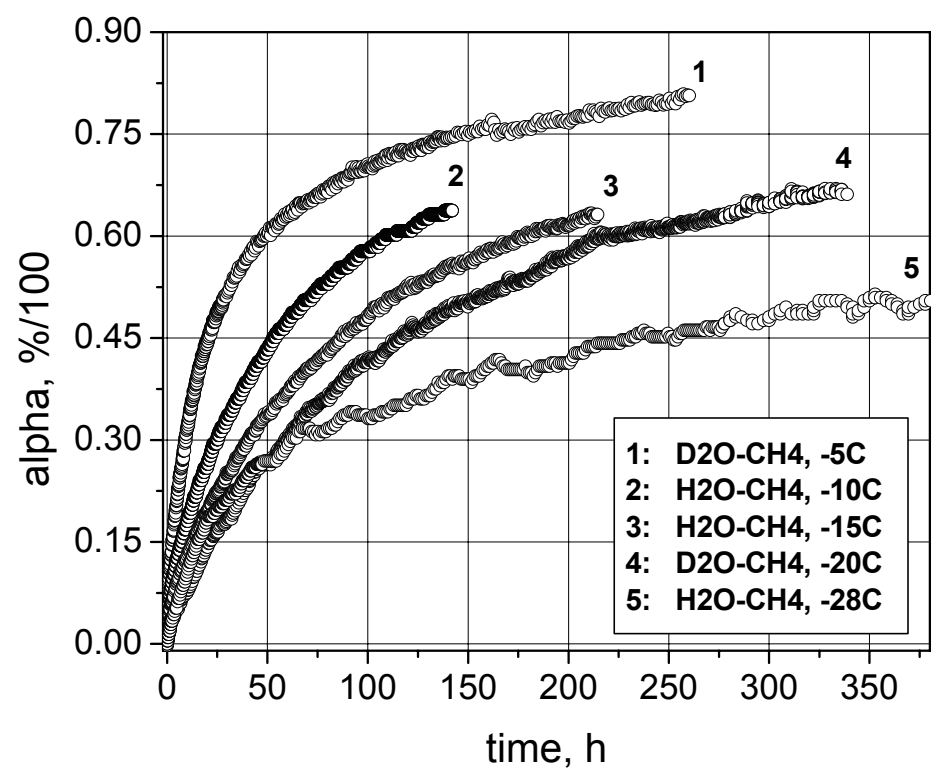

Figure 7. Plot of the clathrate fraction (alpha) versus time obtained for $\mathrm{CH}_{4}$-gas hydrate growth in deuterated and hydrogenated systems at pressure of $6.0 \mathrm{MPa}$ and temperatures between $-28^{\circ} \mathrm{C}$ and $-15^{\circ} \mathrm{C}$. The oscillatory behaviour at $-\mathbf{2 8}^{\circ} \mathrm{C}$ came from the fluctuations of the ambient temperature which were not corrected in the pressure reading (see for details $\mathrm{Ch}$. 3.3)

At first glance, a comparison of the runs showed, for example, about $45 \%$ of methane clathrate was formed from $\mathrm{H}_{2} \mathrm{O}$-ice for about $230 \mathrm{~h}, 87 \mathrm{~h}$ and $53 \mathrm{~h}$ at $-28^{\circ} \mathrm{C},-15^{\circ} \mathrm{C}$ and $-10^{\circ} \mathrm{C}$, respectively. The same amount of gas hydrate was grown from $\mathrm{D}_{2} \mathrm{O}$-ice for $117 \mathrm{~h}$ and $20 \mathrm{~h}$ at $-20^{\circ} \mathrm{C}$ and $-5^{\circ} \mathrm{C}$, respectively. This result was in agreement with the tendency of a decrease of the formation period with an increase of the temperature obtained above for $\mathrm{H}_{2} \mathrm{O}$ systems. Thus, the first estimate of an increase of the clathrate fraction formed per unit time with an increase of the temperature from $-28^{\circ} \mathrm{C}$ to $-5^{\circ} \mathrm{C}$ showed that the temperature could play a major role on the formation kinetics. The isotope difference between $\mathrm{D}_{2} \mathrm{O}$ and $\mathrm{H}_{2} \mathrm{O}$ in the reacting systems did not change dramatically the kinetic curves suggesting that this factor plays a minor role in the process of methane hydrate growth.

Methane gas hydrate formation runs performed at constant temperature and different pressures showed a pressure (or fugacity) dependence of the growth kinetics. The reactions at temperature of $-10^{\circ} \mathrm{C}$ and gas pressure of $9.0 \mathrm{MPa}, 6.0 \mathrm{MPa}$ and 3.0MPa were compared (see Fig. 8). 


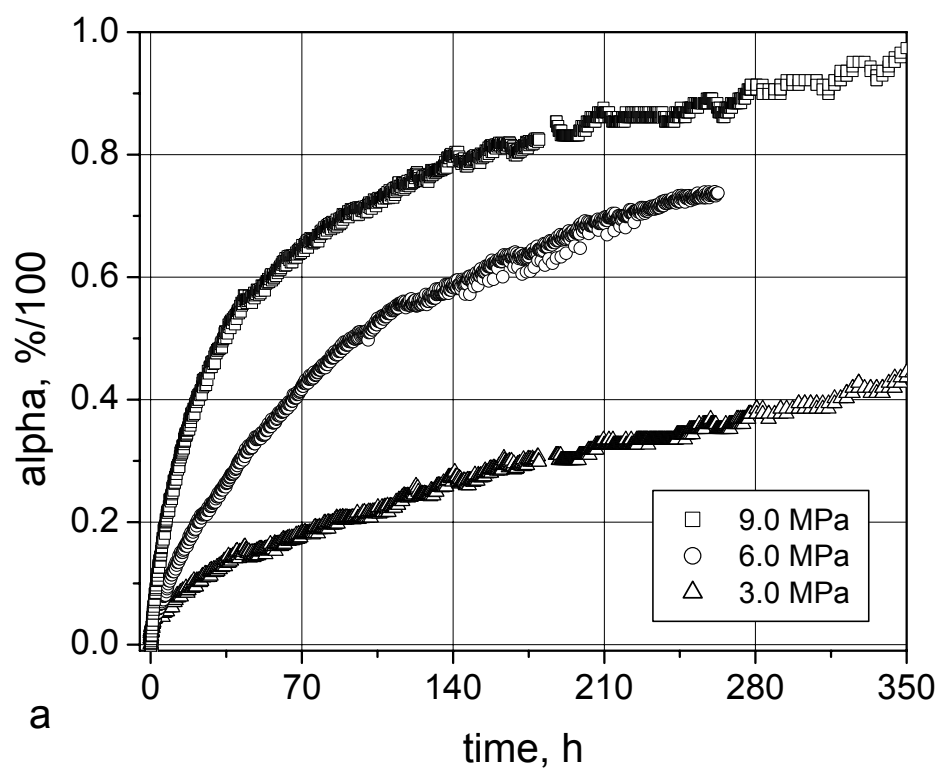

Figure 8. Plot of methane hydrate formation at temperature of $-10^{\circ} \mathrm{C}$ and decreasing pressure of $9 \mathrm{MPa}$, $6 \mathrm{MPa}$ and $3 \mathrm{MPa}$, respectively (runs \#9, \#5 and \#8, Table 1, Ch. 3.3). The obvious oscillatory behaviour of the clathrate fraction at $9 \mathrm{MPa}$ is caused by pressure readings, which were not corrected for ambient temperature changes.

The limited number of formation runs of methane hydrate at different pressures gave only a first idea for a possible role of the excess fugacity in the growth rate. It was observed, for example, that $40 \%$ of methane clathrate was formed for about $22 \mathrm{~h}, 66 \mathrm{~h}$ and $320 \mathrm{~h}$ at $9.0,6.0$ and $3.0 \mathrm{MPa}$, respectively. Correspondingly the excess fugacity was 3.04, 1.92 and 0.58 (see Table 1). Thus, at 5- and 3- times higher fugacity then the lowest one (at 3.0 MPa), the formation time was reduced $15(320 \mathrm{~h} / 22 \mathrm{~h})$ and $5(320 \mathrm{~h} / 66 \mathrm{~h})$ times, respectively.

Deuterated and hydrogenated ice powder was used as a starting material for reactions performed at equivalent thermodynamic conditions. A slightly faster formation of methane clathrate from $\mathrm{H}_{2} \mathrm{O}$ - with respect to $\mathrm{D}_{2} \mathrm{O}$-system was obtained at temperature of $-10^{\circ} \mathrm{C}$ and pressure of 6.0 MPa (see Fig. 9). Such a result could be expected because of the shift in the $\mathrm{H}_{2} \mathrm{O}$ and $\mathrm{D}_{2} \mathrm{O}$ melting points. Still, the isotope difference plays a minor role on the growth rate of methane clathrate, at least at temperatures well below the melting point. 


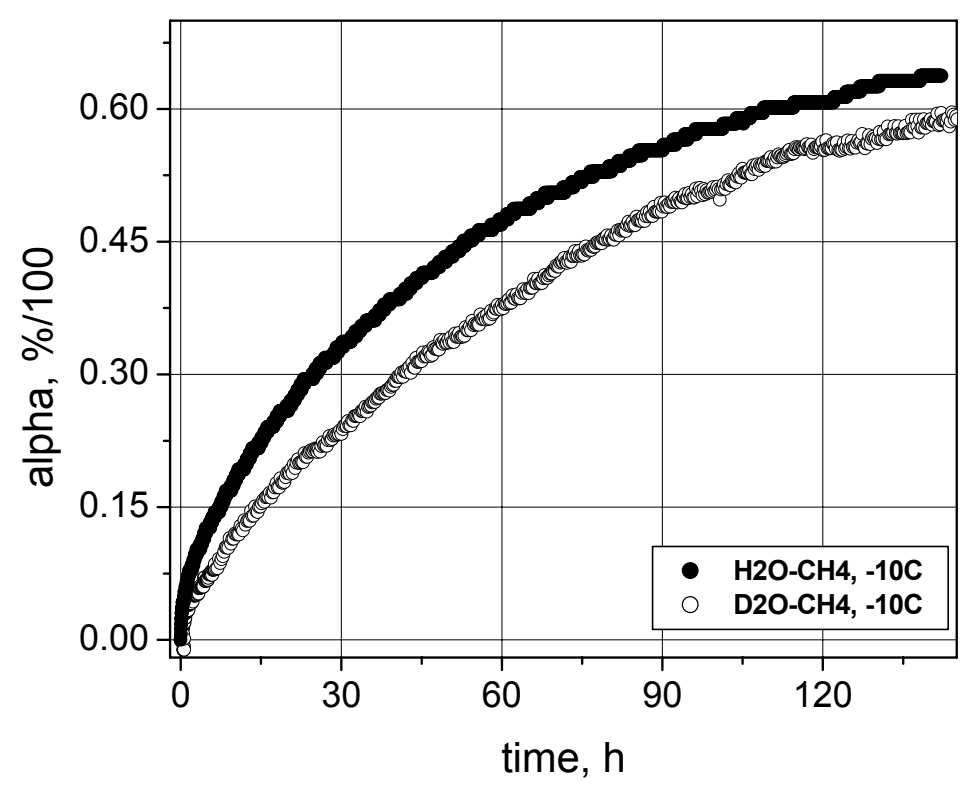

Figure 9. Comparison of $\mathrm{H}_{2} \mathrm{O}-\mathrm{CH}_{4}$ and $\mathrm{D}_{2} \mathrm{O}-\mathrm{CH}_{4}$ growth at temperature of $-10^{\circ} \mathrm{C}$ and pressure of $6.0 \mathrm{MPa}$ (runs \#4, \#5, Table 1, Ch. 3.3).

Gas hydrate formation runs at temperatures very close to the melting point of ice had problems because of largely un-controlled changes of the ice microstructure at these temperatures. As it was mentioned before (Ch. 3.3.4) using the idea of the clathrate formation in different steps (Ch.4) a trial to form a hydrate covering the ice powder (stage I) before being exposed to high temperature conditions was made and reactions at temperatures close to the melting point of ice were run. A set of $\mathrm{H}_{2} \mathrm{O}$-ice samples was reacted at pressure of $6.0 \mathrm{MPa}$ and temperature of $-7^{\circ} \mathrm{C}$ for about $4 \mathrm{~h}$. The obtained samples were used as a starting material for a set of high temperature kinetic runs and the results of the forming gas hydrate were compared (see Fig. 10). The methane clathrate growth turned out to be insensitive to a temperature in the range from -3 to $-1{ }^{\circ} \mathrm{C}$. The figure shows the $\mathrm{CH}_{4}$-gas hydrate grew slower at temperatures higher or equal to $-3^{\circ} \mathrm{C}$ than at lower temperature of $-5^{\circ} \mathrm{C}$. The suppression of the clathrate growth at increasing temperatures was most likely a result of a decrease of the ice surface occurring close to the melting point. The rate of $\mathrm{CH}_{4}$-gas hydrate growth at temperature of $-5^{\circ} \mathrm{C}$ was slower in the hydrogenated system than in the deuterated one reacting at about $5^{\circ} \mathrm{C}$ and $9{ }^{\circ} \mathrm{C}$ below the ice melting point, respectively. Thus, it was not possible to deduce the role of the isotope difference, independently of the one of the changing ice surface, at a temperature of $-5^{\circ} \mathrm{C}$. 


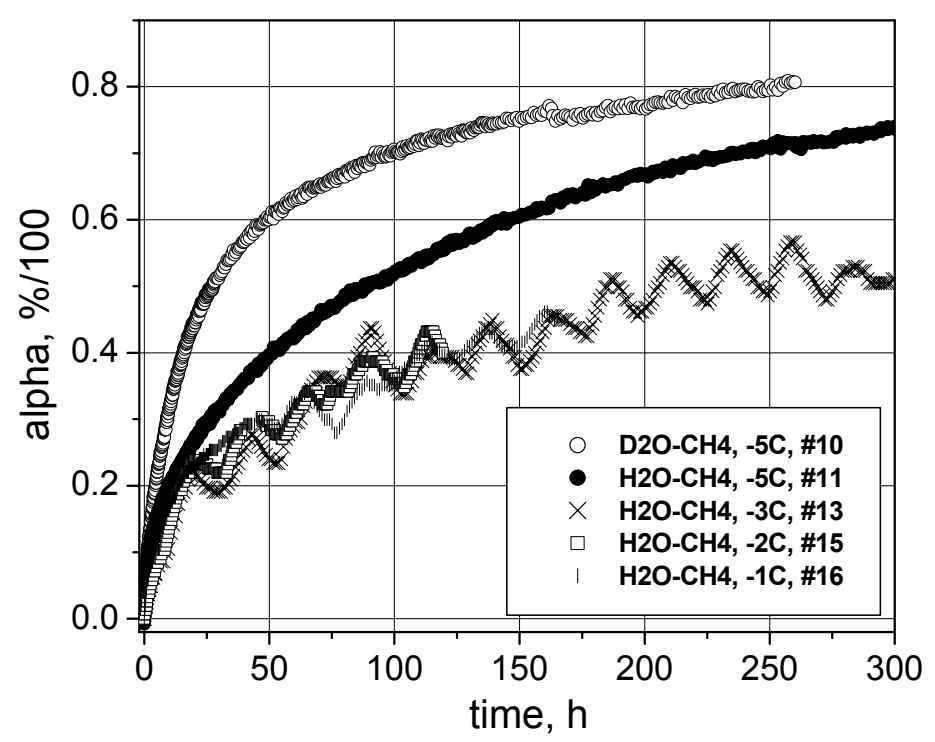

Figure 10. Plots of $\mathrm{CH}_{4}$-gas hydrate formation runs at pressure of 6.0 MPa and temperatures close to the melting point of ice (Table 1, Ch. 3.3). The oscillatory behaviour of the clathrate fraction at temperatures of 270-272K results from a missing ambient temperature correction for the kinetic runs.

Even though the ice was pre-reacted to form an initial clathrate cover, the high temperature growth was proceeding in a clear contrast to the expected increase of the growth rate with an increase of the temperature.

\subsubsection{Multistage Model Fit of Data - Results and Discussion}

As it was mentioned before the process of gas hydrate growth from ice, observed by different experimental methods (Ch.3), was described theoretically by a multistage model (Ch. 4). Mathematical simulations of the process of methane clathrate growth at different thermodynamic conditions were performed as it is described in Appendix G. Thus, the geometrical evolution of an ice sample during a reaction was described by the change of the relative ice core radius $\bar{r}_{i} / \bar{r}_{0}{ }^{*}$, used to calculate the reaction degree. The best fits to the experimental data gave kinetic parameters of the simulated multistage processes of gas hydrate growth (Table 1).

$* \bar{r}_{0}$ and $\bar{r}_{i}$ were initial and current mean-volume radii of ice cores 


\begin{tabular}{|c|c|c|c|c|c|c|c|c|c|}
\hline \multicolumn{5}{|c|}{ Conditions of experiments } & \multicolumn{5}{|c|}{ Parameters of reaction $\left(k_{R}\right)$ and diffusion $(D)$ limited kinetics } \\
\hline $\begin{array}{l}\text { Ice-Gas } \\
\text { System }\end{array}$ & $\begin{array}{l}T, \\
\mathbf{K}\end{array}$ & $\begin{array}{l}P(f) \\
\text { MPa }\end{array}$ & $\begin{array}{c}P_{d}\left(f_{d}\right) \\
\mathbf{M P a}\end{array}$ & $\begin{array}{c}\text { Duration } \\
\text { h }\end{array}$ & $\begin{array}{l}k_{S} \\
1 / \mathbf{h}\end{array}$ & $\begin{array}{c}k_{R} \\
\mathrm{kmol} / \mathrm{m}^{2} \mathrm{~h}\end{array}$ & $\underset{\mathbf{m}^{2} / \mathbf{h}}{D}$ & $F=\frac{D \rho_{i}}{\bar{r}_{0} k_{R}}$ & $\begin{array}{c}\delta_{0}, \\
\mu \mathbf{m}\end{array}$ \\
\hline $\mathrm{D}_{2} \mathrm{O}-\mathrm{CO}_{2}$ & 272 & $2.0(1.73)$ & $1.08(1.00)$ & 18 & 0.95 & 0.0015 & $2 \mathrm{E}-11$ & 0.01 & 7.0 \\
\hline $\mathrm{D}_{2} \mathrm{O}-\mathrm{CH}_{4}$ & 272 & $3.5(3.22)$ & $2.5(2.36)$ & 23 & (1) 0.8 & (1) $1.3 \mathrm{E}-5(>0.8 \mathrm{E}-3)$ & (1) $>1.0 \mathrm{E}-10(1.4 \mathrm{E}-12)$ & (1) $8.2(0.002)$ & (1) $3.5(3.0)$ \\
\hline $\mathrm{D}_{2} \mathrm{O}-\mathrm{CH}_{4}$ & 272 & $6.0(5.20)$ & $2.5(2.36)$ & 18 & (1) 0.8 & (1) $1.5 \mathrm{E}-5(>0.83 \mathrm{E}-3)$ & (1) $>1.0 \mathrm{E}-10(1.45 \mathrm{E}-12)$ & (1) $7.1(0.002)$ & (1) $3.3(2.8)$ \\
\hline $\mathrm{D}_{2} \mathrm{O}-\mathrm{CH}_{4}$ & 268 & $6.0(5.16)$ & $2.19(2.07)$ & 8 & (1) 0.45 & (1) $1.0 \mathrm{E}-5(>0.1 \mathrm{E}-3)$ & (1) $>1.0 \mathrm{E}-10(8.2 \mathrm{E}-13)$ & (1) $13.9(0.011)$ & (1) $3.2(3.0)$ \\
\hline $\mathrm{D}_{2} \mathrm{O}-\mathrm{CH}_{4}$ & 230 & $3.5(3.02)$ & $0.59(0.58)$ & 11.5 & $\begin{array}{l}{ }^{(1)} 0.02 \\
\text { (2) } 0.0145\end{array}$ & (1) & ( & $\begin{array}{l}- \\
-\end{array}$ & $\begin{array}{l}\text { (1) } 3.2(3.2) \\
\text { (2) } 1.6\end{array}$ \\
\hline $\mathrm{D}_{2} \mathrm{O}-\mathrm{CH}_{4}$ & 230 & $6.0(4.62)$ & $0.59(0.58)$ & 11 & $\begin{array}{l}\text { (1) } 0.02 \\
\text { (2) } 0.012\end{array}$ & $\begin{array}{l}- \\
-\end{array}$ & $\begin{array}{l}- \\
-\end{array}$ & $\begin{array}{l}- \\
-\end{array}$ & $\begin{array}{l}\text { (1) } 2.5(2.5) \\
\text { (2) } 1.6\end{array}$ \\
\hline $\mathrm{H}_{2} \mathrm{O}-\mathrm{CH}_{4}$ & 245 & $6.0(4.87)$ & $1.03(0.996)$ & 839 & 0.02 & $1.4 \mathrm{E}-6$ & $1.3 \mathrm{E}-13$ & 0.13 & 5.5 \\
\hline $\mathrm{D}_{2} \mathrm{O}-\mathrm{CH}_{4}$ & 253 & $6.0(4.99)$ & $1.34(1.29)$ & 340 & 0.04 & $4.5 \mathrm{E}-6$ & $7.4 \mathrm{E}-13$ & 0.23 & 2.9 \\
\hline $\mathrm{H}_{2} \mathrm{O}-\mathrm{CH}_{4}$ & 258 & $6.0(5.05)$ & $1.57(1.50)$ & 215 & 0.1 & $6.8 \mathrm{E}-6$ & $1.0 \mathrm{E}-12$ & 0.20 & 2.7 \\
\hline $\mathrm{H}_{2} \mathrm{O}-\mathrm{CH}_{4}$ & 263 & $6.0(5.11)$ & $1.84(1.75)$ & 142 & 0.2 & $1.0 \mathrm{E}-5$ & $2.5 \mathrm{E}-12$ & 0.35 & 2.7 \\
\hline $\mathrm{D}_{2} \mathrm{O}-\mathrm{CH}_{4}$ & 263 & $6.0(5.11)$ & $1.84(1.75)$ & 263 & 0.2 & 7.2E-6 & $1.9 \mathrm{E}-12$ & 0.37 & 1.8 \\
\hline $\mathrm{D}_{2} \mathrm{O}-\mathrm{CH}_{4}$ & 263 & $6.0(5.11)$ & $1.84(1.75)$ & 263 & 0.2 & $5.5 \mathrm{E}-6$ & $1.9 \mathrm{E}-12$ & 0.48 & 1.5 \\
\hline $\mathrm{D}_{2} \mathrm{O}-\mathrm{CH}_{4}$ & 263 & $6.0(5.11)$ & $1.84(1.75)$ & 214 & 0.2 & $8.1 \mathrm{E}-6$ & $1.8 \mathrm{E}-12$ & 0.31 & 1.6 \\
\hline $\mathrm{D}_{2} \mathrm{O}-\mathrm{CH}_{4}$ & 263 & $6.0(5.11)$ & $1.84(1.75)$ & 104.5 & 0.2 & $4.7 \mathrm{E}-6$ & $1.35 \mathrm{E}-12$ & 0.40 & 1.9 \\
\hline $\mathrm{D}_{2} \mathrm{O}-\mathrm{CH}_{4}$ & 263 & $3.0(2.77)$ & $1.84(1.75)$ & 506 & 0.2 & $3.0 \mathrm{E}-6$ & 7.2E-13 & 0.33 & 2.1 \\
\hline $\mathrm{D}_{2} \mathrm{O}-\mathrm{CH}_{4}$ & 263 & $9.0(7.07)$ & $1.84(1.75)$ & 506 & 0.2 & $2.5 \mathrm{E}-5$ & $1.9 \mathrm{E}-12$ & 0.11 & 2.9 \\
\hline $\mathrm{D}_{2} \mathrm{O}-\mathrm{CH}_{4}$ & 268 & $6.0(5.16)$ & $2.19(2.07)$ & 260 & 0.4 & $7.5 \mathrm{E}-5$ & $2.4 \mathrm{E}-12$ & 0.04 & 4.0 \\
\hline $\mathrm{D}_{2} \mathrm{O}-\mathrm{CH}_{4}$ & 270 & $6.00(5.19)$ & $2.33(2.20)$ & 135.4 & 0.7 & 9E-5 & $1.35 \mathrm{E}-12$ & 0.02 & 2.2 \\
\hline
\end{tabular}

Table 1. Experimental conditions and kinetic parameters deduced by fitting with the polydisperse multistage model. The short-term kinetic data (up to 24h) were modelled without crack filling (Ch. 4.2.4) and the obtained parameters are referenced under (1). They showed that the initial clathrate growth, recorded for a short time, could be described by parameters of either a reaction-limited (without brackets) or a diffusion-limited kinetics (with brackets) and the given values should be regarded as an order of magnitude of $k_{R}$ and $D$ for both possible cases. The model including the crack filling (Ch.4.2.2) was used to fit the data at $230 \mathrm{~K}$ and the kinetic parameters are referenced under (2). 
The polydisperse model assumes that the surface coating was the only process, taking place in the first stage of the reaction. Ignoring the crack filling sub-stage (Ch. 4.2.2) led to a discrepancy between the observation and model fit at the very initial part of the reaction. With the decrease of the temperature the initial misfit became more pronounced as it is shown in Fig. 11, for example, with the best fits found at $6.0 \mathrm{MPa}$ and the temperatures of 272, 270 and $263 \mathrm{~K}$.

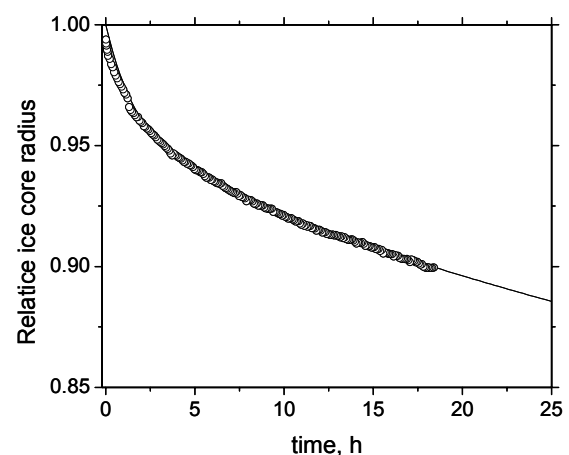

a

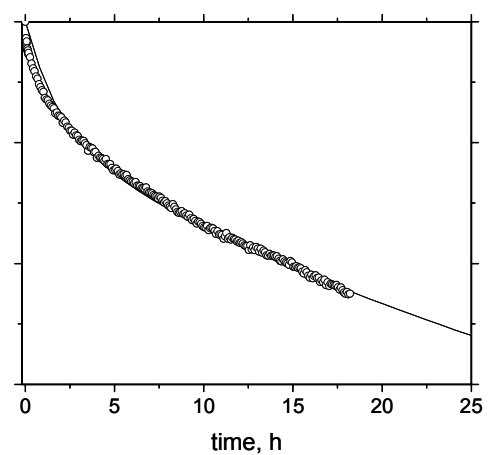

b

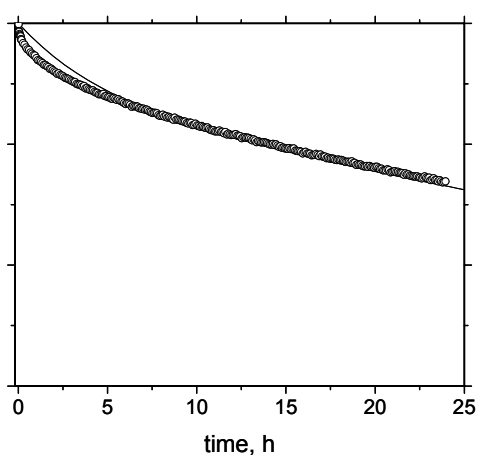

C

Figure 11. Model predictions (solid lines) fitted to the neutron diffraction data of methane hydrate formation (circles) at 6.0 MPa and 272, 270 and 263K (plots a-c, respectively).

The typical time scale $\left(k_{S}^{-1}\right)$ of the surface coating (stage I) had grown from about $1.4 \mathrm{~h}$ to approximately 2-3 days as the temperature decreased from 270 to $230 \mathrm{~K}$ (see Table 1). The kinetic data of methane hydrate growth with a reaction degree not exceeding 20-30\% were equally well fitted by the model simulations either of a reaction- or a diffusion-limited process (see Table 1, the values referenced under (1)). This is further discussed below. The fits of the short-term reactions at $230 \mathrm{~K}$ were insensitive to changes in $k_{R}$ and $D$ and thus, only the surface coating parameters were deduced. In the light of low-temperature SEM observations of $\mathrm{CO}_{2}$ clathrate formation, a crack filling sub-stage was distinguished from the later surface coverage (Genov et al., 2004). The SEM studies of methane hydrate formation at high temperature suggested that the filling of the cracks in the ice grains occurred more or less simultaneously with the necking of adjacent particles as well as the formation and lateral spreading of hydrate patches on the spherical ice grain surface. A simultaneous treatment of these effects in the fitting of the model to the high temperatures data sets caused problems due to parameter correlations. 
Therefore, only a re-analysis of the low-temperature neutron data was done using the extended model with a crack-filling stage, which had become available only in the final stage of this work (Fig. 12, Table 1, the values referenced under (2)).
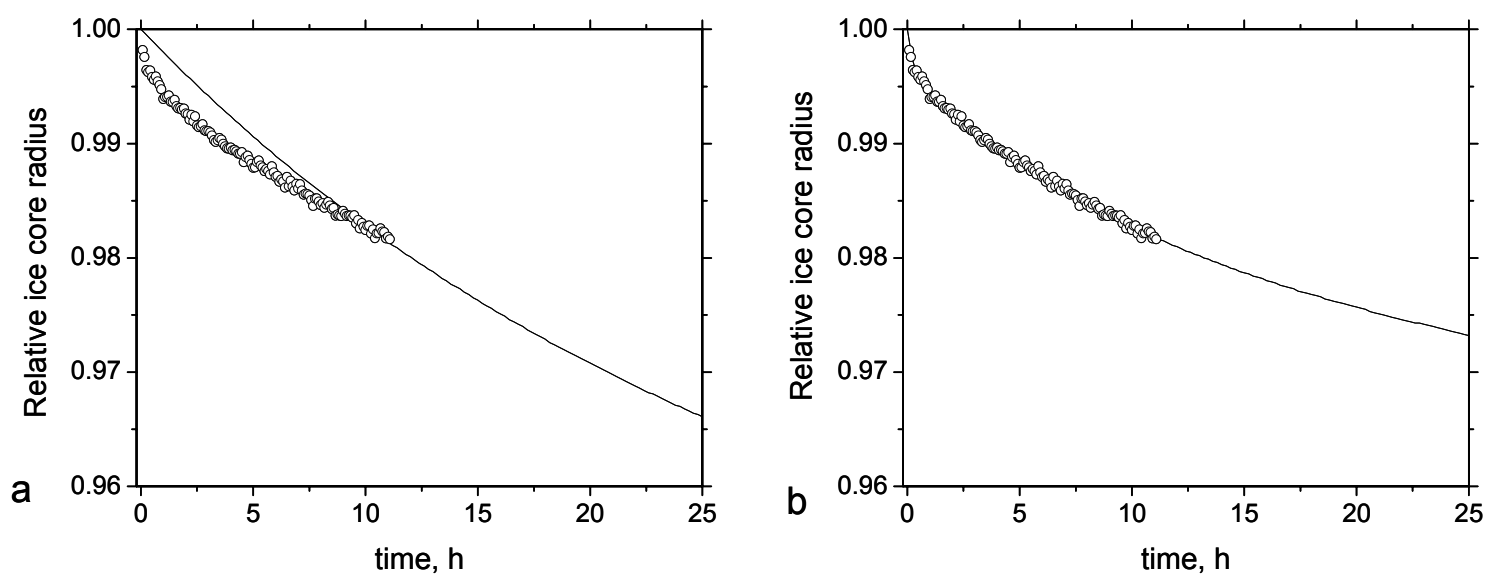

Figure 12. Model predictions (solid lines) of the polydisperse model not simulating (a) and simulating (b) a crack-filling sub-stage plotted together with the data of the initial growth of $\mathrm{CH}_{4}-\mathrm{D}_{2} \mathrm{O}$ hydrate at $6.0 \mathrm{MPa}$ and 230K. The misfit of the very initial part of the reaction observed in (a) was improved when the crack filling was included (b).

The reactions at $230 \mathrm{~K}$ were interpreted as pure crack fillings (Fig. 12b) during the first half an hour $\left(k_{S}\right.$ ' 2), followed by the surface coatings. Transitions to the following stages (II and III) of the reactions were not observed because of the short period (about $11 \mathrm{~h}$ ) of the measurements.

The transition of the surface coating to the later reaction- and diffusion-limited stages of $\mathrm{CH}_{4}$-clathrate formation was obtained by fits of the neutron-diffraction and gas-consumption data recorded for periods of days to weeks. Figure 13 gives examples of best fits found for diffraction and consumption data at temperatures of $270,263 \mathrm{~K}$ and $253,268 \mathrm{~K}$, respectively, and constant pressure of 6.0 MPa. 

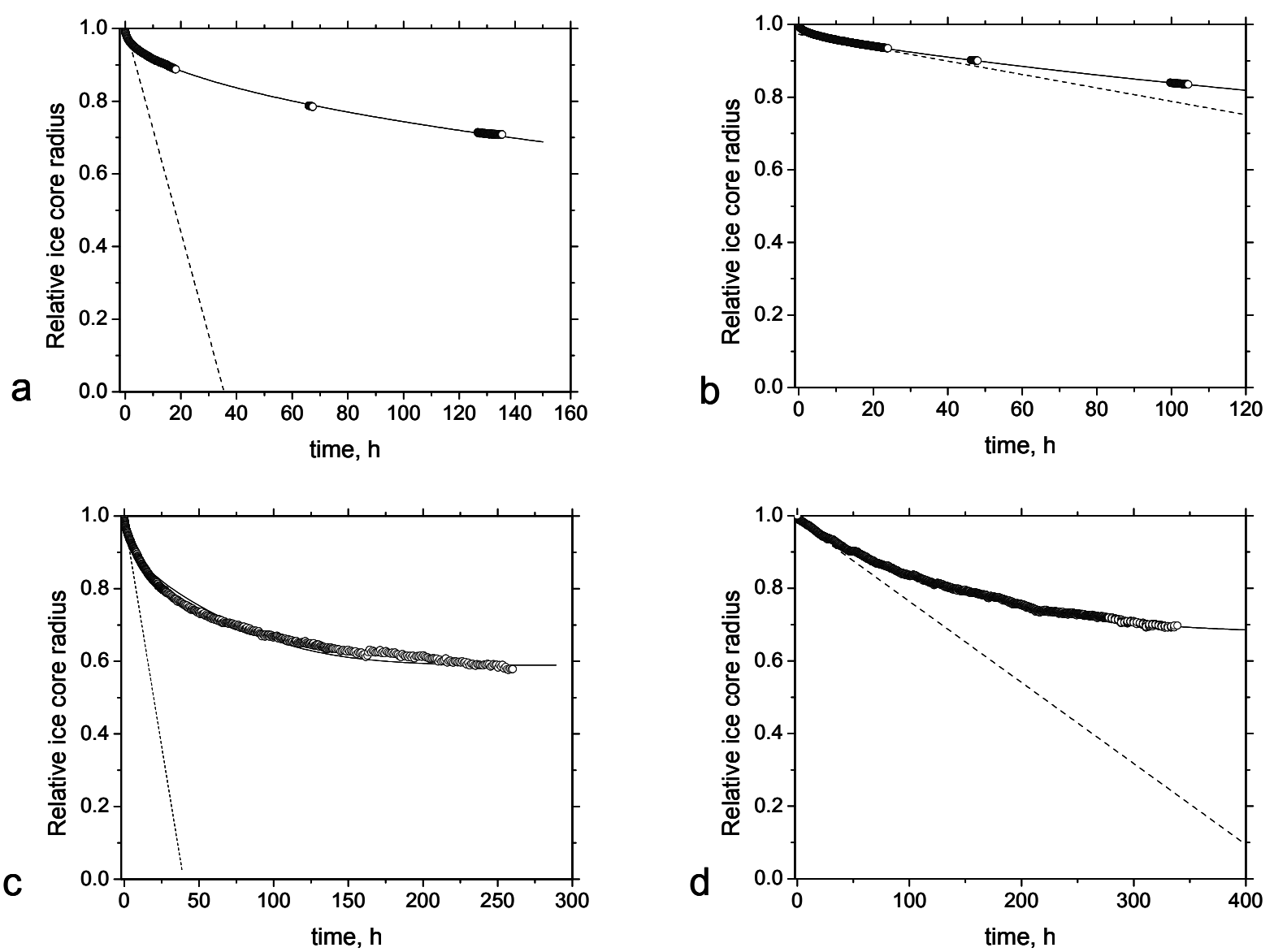

Figure 13. Model predictions (solid lines) of $\mathrm{CH}_{4}-\mathrm{D}_{2} \mathrm{O}$ formations at 6.0 Mpa, which were measured (circles) by neutron-diffraction (plots (a)-(b)) and gas-consumption (plots (c)-(d)) methods. The dashed lines, corresponding to a linear approximation of Eq. 8 for stage II (see Ch. 4.2.4), indicated the reactions at higher temperatures of $270 \mathrm{~K}$ and $268 \mathrm{~K}$ (plots (a) and (c), respectively) reached the diffusion-limited stage faster then the ones at lower temperatures of $263 \mathrm{~K}$ and $253 \mathrm{~K}$ (plots (b) and (d), respectively).

Each dashed line was a simulation of an ideal reaction-limited process (no diffusion limitation). The reactions observed by both methods underwent a transition to a diffusion-limited stage (shown by the point of discrepancy between the dashed and solid lines) faster at a higher temperature then at a lower one. One similar tendency of a late and later transition to the diffusion-controlled step on lowering the pressure (Fig. 14) was observed at 9.0, 6.0 and 3.0MPa and $263 \mathrm{~K}$. 


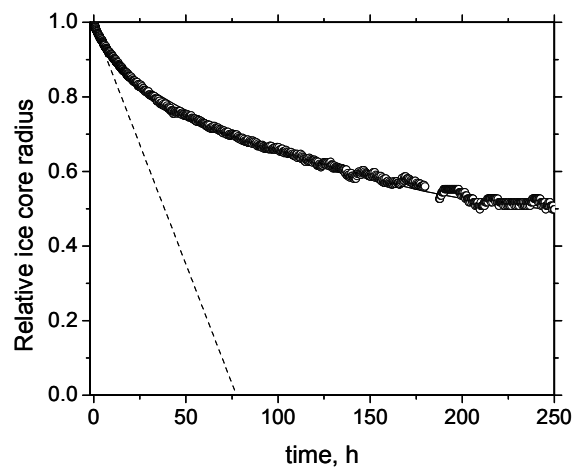

a

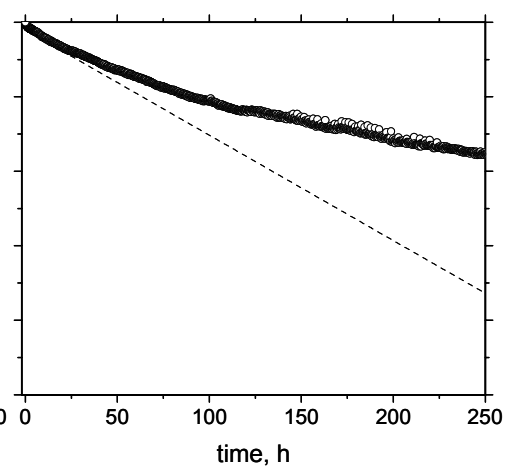

b

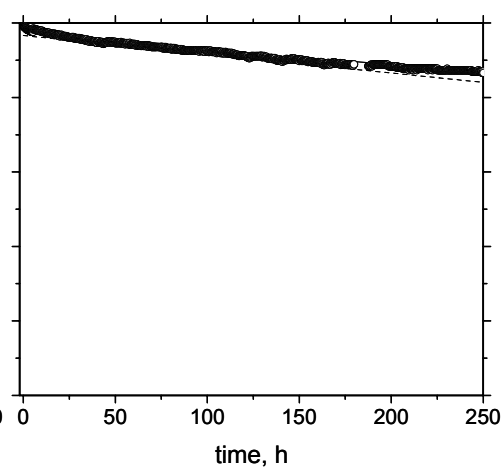

C

Figure 14. The transition to stage III proceeded faster at higher pressure then at lower ones as it is indicated by the discrepancies of the dashed lines (stage II) and the plots of the model predictions (solid lines) fitted to the data of $\mathrm{CH}_{4}-\mathrm{D}_{2} \mathrm{O}$ formation (circles) of 9.0 MPa, 6.0 MPa and 3.0 MPa (plots (a), (b) and (c), respectively).

A dimensionless diffusion-to-reaction factor $F$ (Eq. (10), Ch.4.2.4) contains information on the onset of stage III limited by the gas/water mass transfer through the hydrate shells. Large values of $F$ mean that the reacting system could never transit to stage III. On the opposite, values of $F<<1$ correspond to an extremely short stage II, which would overlap with stage I and would be almost immediately replaced by a diffusion-limited stage III. As it was discussed above the short-term kinetics of methane hydrate growth of less than $30 \%$ could be equally well fitted by polydisperse-model predictions either for reaction- $(F \sim 10-100)$ or diffusion-limited $\left(F \sim 10^{-3}-10^{-2}\right)$ process (see Table 1 , the values referenced under (1)). Long-term runs at $\mathrm{T}<$ $268 \mathrm{~K}$ gave intermediate values of $F \sim 1$, describing the onset of stage III as simultaneously controlled by both processes of reaction and diffusion. The reactions performed at $\mathrm{T} \geq 268 \mathrm{~K}$, giving values of $F \sim 10^{-2}$, suggested that the high temperature kinetics of $\mathrm{CH}_{4}$ - as well as $\mathrm{CO}_{2}$ clathrate growth was most likely controlled by diffusion.

The best fits of $\mathrm{CH}_{4}$-clathrate growth showed a temperature dependence of the rate constants of the surface coating $\left(k_{S}\right)$, reaction-limited $\left(k_{R}\right)$ and diffusion-limited $(D)$ stages of the process. Thus, the formation of methane gas hydrate appears to be an activated process within the investigated temperature interval for which an Arrhenius behavior of the rate constants was assumed. As it was shown before (Ch. 5.1.2) the shift between the $\mathrm{D}_{2} \mathrm{O}$ and $\mathrm{H}_{2} \mathrm{O}$ melting points influenced the gas hydrate kinetics at very high temperatures. At lower temperatures the difference in the reactions from $\mathrm{D}_{2} \mathrm{O}$ - and $\mathrm{H}_{2} \mathrm{O}$ - ice was barely significant. Thus, the limited 
number of values deduced for hydrogenated systems were combined with the ones for deuterated systems in the Arrhenius plots. When an activation energy $Q_{S}$ of the surface coverage stage of $\mathrm{CH}_{4}$-clathrate formation was extracted two different temperature regions, above and below $250 \mathrm{~K}$ $(1 / \mathrm{T}=0.004)$, had to be considered. For the higher and lower temperature region the values of the activation energy were found as $89.6 \pm 3.1$ and $12.8 \pm 5.0 \mathrm{~kJ} / \mathrm{mol}$, respectively (Fig. 15a). The higher activation energy may be connected to changes of the ice surface occurring at temperatures approaching the melting point of ice (Ch. 2.3.1). Nevertheless, more experimental data at low temperatures are necessary to clarify this. The activation energy of $\mathrm{CH}_{4}$ - hydrate formation on the assumption of a reaction-limited process (stage II) was found as $86.2 \pm 17.1$ $\mathrm{kJ} / \mathrm{mol}$ (Fig. 15b). The variation of $k_{R}$ at $6.0 \mathrm{MPa}$ and $263 \mathrm{~K}$ (Table 1) suggested that the stage II was not well determined within the kinetic curves. Thus, the transition between stage I and stage III at these conditions was somewhat smeared and the second stage most likely was overlapped by the surface coating and diffusion-limited stages. In this relation it was already mentioned that the FE-SEM (Ch. 3.4.6) observations on the reacted sample showed the reaction proceeded with a different rate in the different parts of the sample. Thus, a global average reaction within the whole sample was measured by the in situ used techniques. In addition the model is an approximate description of the complex geometry of the heterogeneous hydrate reaction. Thus, an uncertainty of $k_{R}$ at one temperature was shown with the plot of an average of all corresponding $\ln \left(k_{R}\right)$ within an interval limited by the maximum and minimum (Fig. 15b). There was no direct measurements of the uncertainties at other temperatures and thus, the one deduced at $263 \mathrm{~K}$ was assumed as a representative of the rest as well. An activation energy of $56.3 \pm 12.9$ $\mathrm{kJ} / \mathrm{mol}$ of stage III (diffusion-limited) of $\mathrm{CH}_{4}$-clathrate growth was obtained (Fig. 15c). Similar activation energy of $14.7 \mathrm{kcal} / \mathrm{mol}(61.3 \mathrm{~kJ} / \mathrm{mol})$ was obtained by Wang et al., 2002 who studied the kinetics of the $\mathrm{CH}_{4}$-hydrate formation from deuterated ice particles as a diffusion-controlled process. Moreover, similar activation energy of a diffusion-limited $\mathrm{CO}_{2}$-clathrate growth from polydisperse ice powder $(54.6 \mathrm{~kJ} / \mathrm{mol})$ was deduced by Genov et al., 2004. 

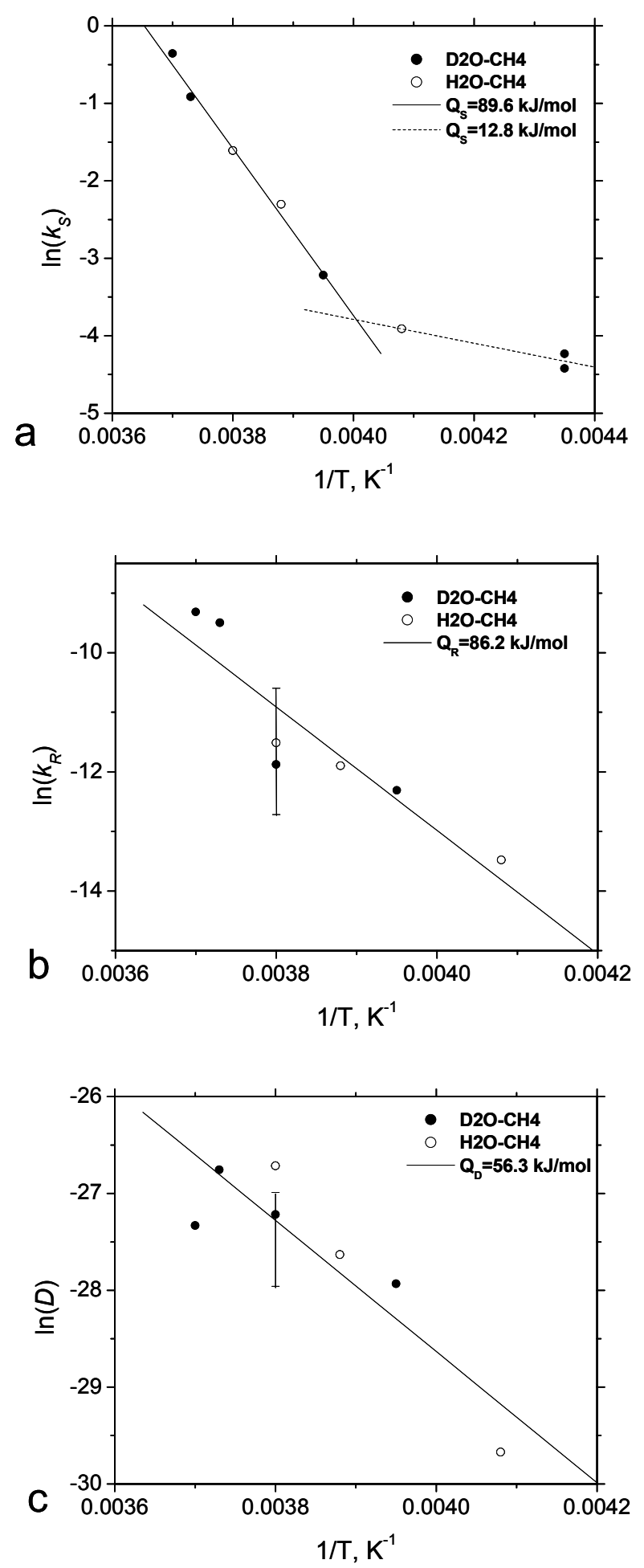

Figure 15. Arrhenius plots of the surface coverage (a), reaction-limited (b) and diffusion-limited (c) stages of $\mathrm{CH}_{4}$-hydrate formation. Black and white circles marked best-fit model parameters obtained for the reactions in deuterated and hydrogenated systems, respectively (see Table 1 , the values denoted with ${ }^{(1)}$ and ${ }^{(2)}$ were not included). Variations of the values of $k_{R}$ and $D$ at $263 \mathrm{~K}$ deduced for $\mathrm{D}_{2} \mathrm{O}-\mathrm{CH}_{4}$ systems were marked with error bars. 


\subsection{Summary}

The collected experimental material on the formation of methane clathrate was accounted for by a phenomenological approach of this multistage process. In all observed $\mathrm{CH}_{4}$-clathrate formation runs the kinetic curve had a strongly non-linear development with time in the initial stage and flattened in the later stages still keeping a smooth overall shape.

A clear difference between $\mathrm{CO}_{2}$ and $\mathrm{CH}_{4}$ clathrate formers at high temperature was established. The isotopic difference between $\mathrm{H}_{2} \mathrm{O}$ and $\mathrm{D}_{2} \mathrm{O}$ was found to be negligible in terms of clathrate formation properties. The reaction curves of $\mathrm{CH}_{4}$-clathrate formation at high temperatures were smooth and made it difficult to discern the transition between the stages. The time scales of the ice surface coating $\left(k_{S}^{-1}\right)$ ranged from several hours to several days, depending on the thermodynamic conditions (see Table 1). The rates of the subsequent stages were deduced from the best data fits and suggested that the methane hydrate formation is a temperature dependent process. The estimations of the activation energies of the different stages were given. The surface coating stage showed a stronger dependence on the temperature (higher activation energy) when the melting point of ice was approached. An obtained change in the activation energy at about $250 \mathrm{~K}$ is a preliminary estimate and needs more experimental data in this temperature region to be corroborated. The smearing of the different stages in the kinetic curves led to variations in the obtained rate constants for stage II and III (see the error bars, Fig. 15b, c). Thus, the transition from stage I to stage III was not well determined within the kinetic curves. This is most likely because of the averaging of the formation process within the whole sample smearing the periods of the different steps as well as the fast proceeding of stage II related to the free transport of gas and water molecules through the hydrate shells. Moreover Georgi Genov (GZG-Göttingen, private discussions), who studied FE-SEM images of the gas hydrate and processed some of them by developed for this image software, deduced a topology corresponding to a foam* microstructure. Likewise, measurements of the specific surface area (SSA) of partly reacted $\mathrm{CH}_{4}$-gas hydrate samples were made (Andreas Zeller, diploma student, GZG-Göttingen) using a BET (Brunauer, Emmet and Teller, 1938) adsorption method originally developed for SSA measurements of snow (Legagneux et al., 2002, Legagneux et al., 2003) in order to check to which extend the nanometric pores are interconnected. The SSA measurements showed an increase of the SSA mainly during the surface coverage stage I reaching a limiting value, which

\footnotetext{
* foam - solution consisting of two phases - dispersed and continuous one.
} 
suggested that, the porosity was only partly open over a scale of a few $\mu \mathrm{m}$. Thus, most likely, only the initial hydrate film spread over the ice-grain surface exhibit a high permeability. Further on, as thicker clathrate layers developed the mass transport at the later stages of the observed clathration reaction most likely occurred by bulk diffusion. 


\section{References}

Brunauer, S.; Emmet, P.H.; Teller, E. (1938). Adsorption of gases in multimolecular layers. $J$. Am. Chem. Soc., 60, 309-319.

Chihaia, V.; Adams, S.; Kuhs, W. F. (2004). Influence of water molecules arrangement on structure and stability of $5^{12}$ and $5^{12} 6^{2}$ buckyball water clusters. A theoretical study. Chemical Physics, 297, 271-287.

Fleyfel, F.; Devlin, J.P. (1991). Carbon dioxide clathrate hydrate epitaxial growth: spectroscopic evidence for formation of the simple Type-II $\mathrm{CO}_{2}$ hydrate. Journal of Physical Chemistry, 95, 3811-3815.

Genov, G.; Kuhs, W. F.; Staykova, D. K.; Goreshnik, E.; Salamatin, A. N. (2004). Experimental studies on the formation of porous gas hydrates. American Mineralogist, Vol. 89, No. 8-9, 1228-1239.

Legagneux, L.; Cabanes, A.; Dominé, F. (2002). Measurement of the specific surface area of 176 snow samples using methane adsorption at 77K. Journal of Geophysical Research, 107, 4335-4349.

Legagneux, L.; Lauzier, T.; Dominé, F.; Kuhs, W.F.; Heinrichs, T.; Techmer, K. (2003). Rate of decay of snow during isothermal experiments and morphological changes studied by scanning electron microscopy. Canadian Journal of Physics, 81, 459-468.

Wang, X.; Schultz A.J.; Halpern Y. (2002) Kinetics of ice particle conversion to methane hydrate. Proceedings of the Fourth International Conference on Gas Hydrates, Yokohama, May 19-23, 2002, 455-460. 


\section{Summary}

The thesis work presented here has to be regarded as a first step to gain quantitative information on the growth kinetics of methane hydrate from ice. For this goal different experimental methods were tested and their possibilities and restrictions to follow in situ the clathrate formation were deduced.

The complex experimental set-up needed for the low-temperature/high-pressure conditions of gas hydrate formation put constraints on the possible techniques of investigation. The neutron powder diffraction method was found to be the best tool for a direct observation of the growth process in particular in the first stage of the reaction. An automatic data processing was developed and optimised to extract the kinetic information. The relative amount of new phase, obtained with a rather high precision, had a meaning of an average value for the entire sample. The method was confined to deuterated systems and the period of investigation limited by the time allocation of neutron facilities to a few days. Nevertheless, the neutron diffraction method extracted results of $\mathrm{CH}_{4}$-hydrate growth at different pressures and temperatures, which was a valuable piece of information for the studied process. The observed clathrate formation was very rapid initially and then slowed down in at least two further stages. In addition, a difference between $\mathrm{CO}_{2}$ and $\mathrm{CH}_{4}$ hydrate formers was established.

The synchrotron X-ray diffraction was the next promising tool for direct in situ observations of the gas hydrate growth from $\mathrm{D}_{2} \mathrm{O}$ - and $\mathrm{H}_{2} \mathrm{O}$ - ice. A number of different problems appeared when the method was tested. The obtained diffraction patterns contained irregular background features and attempts to process the X-ray kinetic data in the way as it was done for the neutron diffraction data, failed. In order to use the synchrotron X-ray diffraction for studies of the gas hydrate kinetics a new approach of the data processing will have to be developed.

Using the high-pressure/low-temperature set-up available in the physico-chemical laboratory of Abt. Kristallography - GZG, Göttingen, a gas consumption method was developed and optimised for in situ measurements of $\mathrm{CH}_{4}$-gas hydrate formation. As a result long-term reactions of few weeks were recorded. Although the gas consumption method was not as precise as neutron diffraction, it provided very important information on the later stages of $\mathrm{CH}_{4}$-hydrate growth and allowed for a comparison of reactions from deuterated and hydrogenated ice. The 
isotopic difference between $\mathrm{H}_{2} \mathrm{O}$ and $\mathrm{D}_{2} \mathrm{O}$ was found to be un-important at low temperature and played some role only on approaching the melting point.

Starting the reactions from well-characterised ice powder allowed the modelling of $\mathrm{CH}_{4}$ hydrate growth from the beginning till the end of the runs. The FE-SEM investigations provided important ex situ observations of the changes occurring during the hydrate formation. The SEM studies led to a possible scenario of a reaction converting an ice powder, consisting of spherical grains, to a consolidated sample of sub-micron porous structure. The FE-SEM played a key role for the development of the phenomenological multistage model describing the clathrate formation process as follows: an initial hydrate-film spreading over the ice surface (stage I) is followed by an inward and outward hydrate growth limited by the clathration reaction at the hydrate-ice interface (stage II) and the diffusion (stage III) of gas and water through the growing hydrate shells.

The in situ kinetic data of $\mathrm{CH}_{4}$-gas hydrate formation were simulated by the multistage model of gas hydrate growth within the entire reaction periods. The obtained kinetic parameters of the different stages of the studied $\mathrm{CH}_{4}$-hydrate formations were used to calculate the activation energies of the three stages of methane clathrate growth.

Although the molecular mechanisms of the gas hydrate formation are not clarified yet, the experimental studies provided valuable quantitative information on the $\mathrm{CH}_{4}$-hydrate growth. Interestingly, the sub-micron structure revealed by the laboratory-made gas hydrates was found undistinguishable from the one of the natural gas-hydrates from the ocean sea floor, mostly formed from liquid water. The modelling of the formation of synthetic methane clathrates resulted in kinetic parameters, forming the basis for an estimation of similar processes occurring in the nature at geological time scales. 


\section{Appendices}




\section{Appendix A.1}
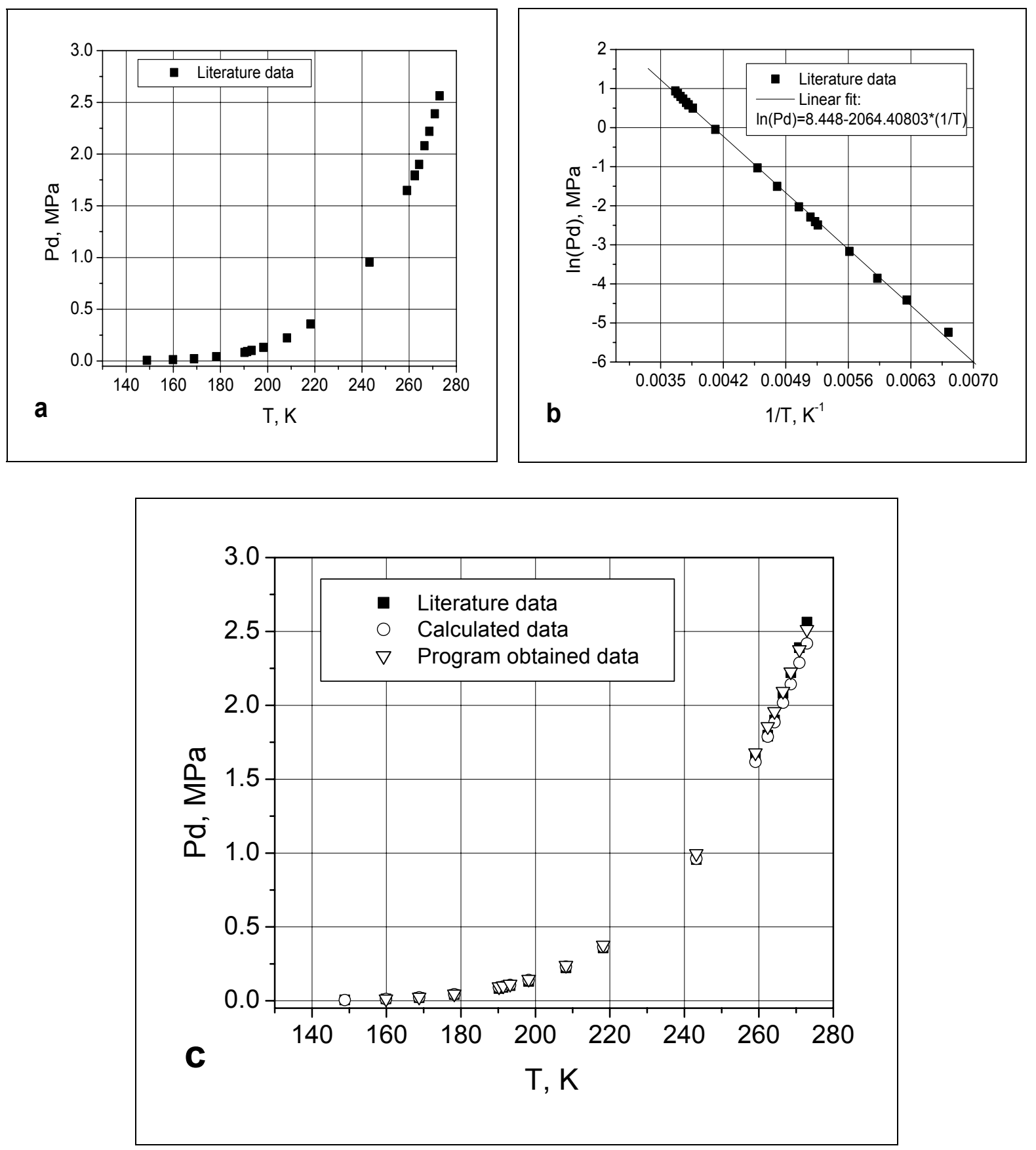

Figure 1. Literature data (Sloan, 1998) (a) were used to obtain a formula for the decomposition pressure $P d$ as a function of temperature $T$ using the linear fit (b) of $\ln (P d)$ as a function of $1 / T$. (c) The $P d$ values calculated by the formula (circles), are compared with the literature values (black squires) and the values calculated by the program of Sloan (CSMHYD.EXE, 1998) ${ }^{1}$ (triangles), giving a good agreement between the experimental and calculated decomposition pressures.

${ }^{1}$ Sloan E. D., Jr. (1998). Clathrate hydrates of natural gases. Second edition, Marcel Dekker Inc.:New York. 


\section{Appendix A.2}

A system of linear equations (1) is used to calculate the values of fugacity $f$ at gas pressures $P$ of the performed kinetic experiments (Ch. 3.1-3.3). $T_{i}, P_{i}$ are values taken from the International thermodynamic tables for $\mathrm{CH}_{4}$ and $\mathrm{CO}_{2}$ fluid (1976) at temperature and pressure which are lower $(i=1)$ and higher $(i=2)$ than the searched ones. The parameters $c_{i j}$ are fugacity/pressure ratios $(f / p)$ taken for a temperature $T_{i}$ and pressure $P_{j}$. The solution of the linear system (1) gives a value of fugacity $f$ at certain pressure $P$ and temperature $T$.

$$
\begin{aligned}
& a_{1}+b_{1} * T_{1}=c_{11} \\
& a_{1}+b_{1} * T_{2}=c_{21} \\
& a_{2}+b_{2} * T_{1}=c_{12} \\
& a_{2}+b_{2} * T_{2}=c_{22} \\
& a_{1}+b_{1} * T=c_{1} \\
& a_{2}+b_{2} * T=c_{2} \\
& m+n * P_{1}=c_{1} \\
& m+n * P_{2}=c_{2} \\
& m+n * P=c \\
& f=c * P
\end{aligned}
$$

For each experimental pressure the linear system (1) is solved by a source code running under Maple 7 software. 


\section{Appendix A.3}

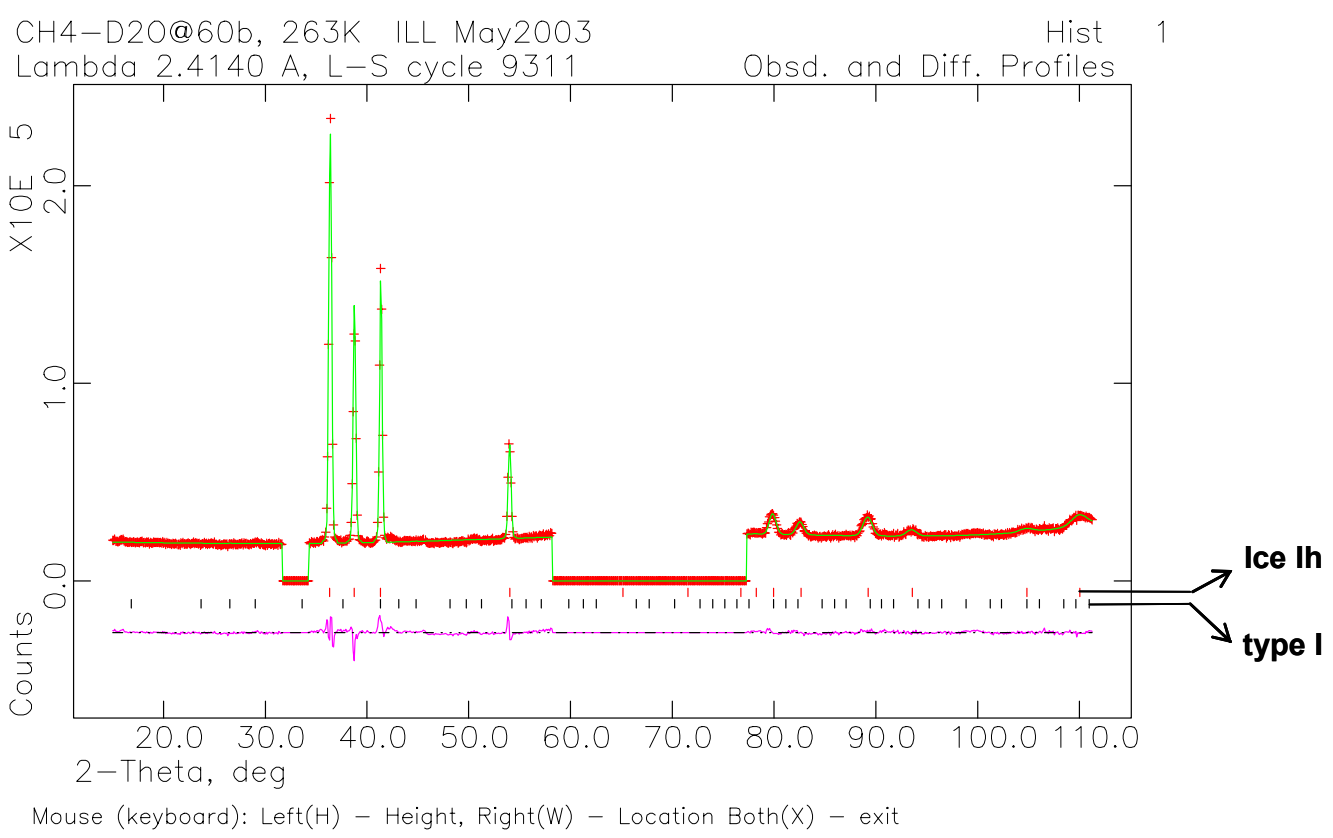

a

Mouse (keyboard): Left(H) - Height, Right(W) - Location Both $(X)$ - exit

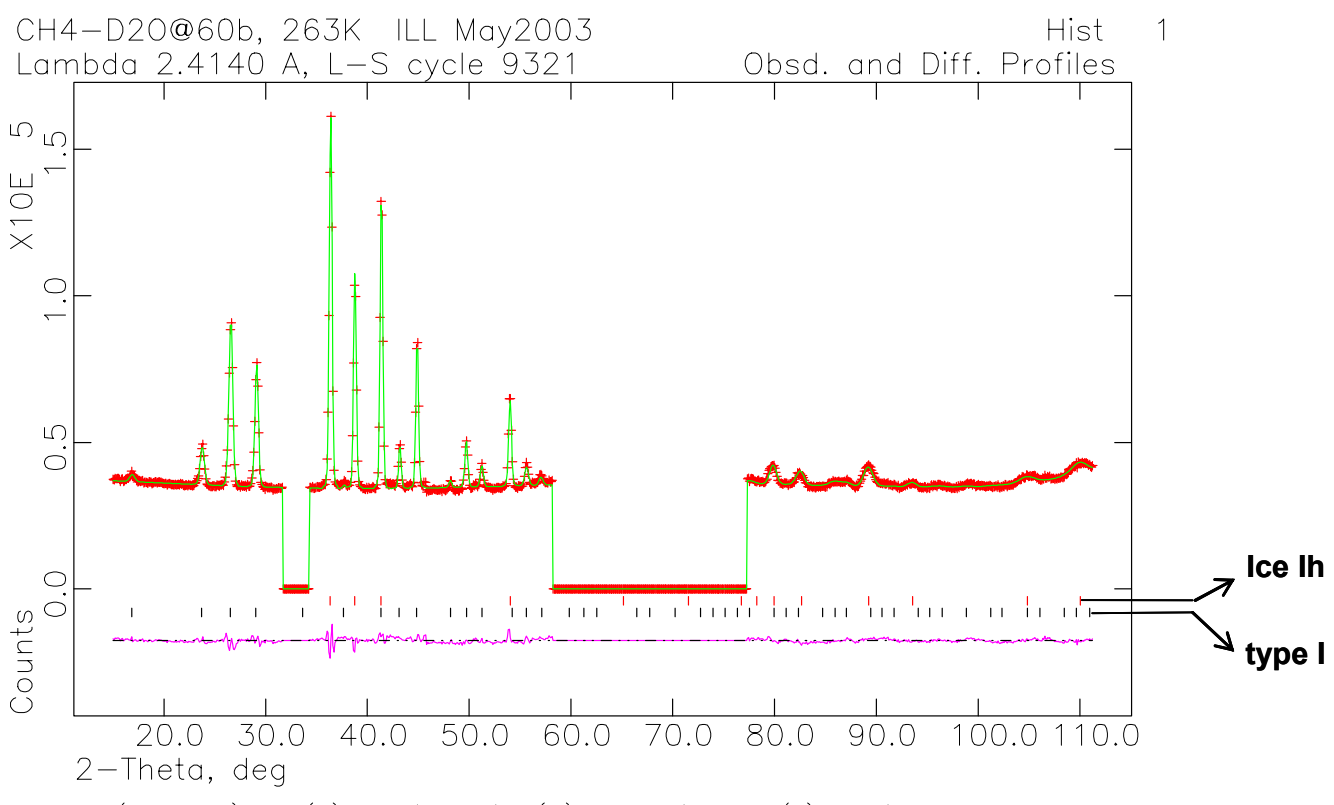

b

Mouse (keyboard): Left(H) - Height, Right(W) - Location Both $(X)$ - exit

Figure 2. GSAS plot of Rietveld fit of the neutron diffraction data of $\mathrm{CH}_{4}-\mathrm{D}_{2} \mathrm{O}$ hydrate formation at 6.0 MPa, $263 \mathrm{~K}$ corresponding to (a) 1 -min data slice obtained after high gas pressure is applied (type-I: $(0.1 \pm 0.4) \%)$ and (b) 5-min data slice obtained after $104.5 \mathrm{~h}$ of reaction (type $\mathrm{I}$ : $(41.8 \pm 0.2) \%)$. The observed and calculated fit values are marked with crosses and a continuous line, respectively. The excluded regions cover the pressure cell scattering. 
Model of type $\mathrm{I}_{2} \mathrm{O}-\mathrm{CH}_{4}$ gas hydrate at $3.5 \mathrm{MPa}$ and $273 \mathrm{~K}$ obtained from neutron diffraction studies (Klapproth, 2002).

\begin{tabular}{cccccc}
\hline \hline \multirow{2}{*}{ Type } & \multicolumn{2}{c}{ Fractional coordinates } & U $\begin{array}{c}\text { Uiso } \mathbf{x 1 0 0}, \\
{\left[\AA^{2}\right]}\end{array}$ & Occ. \\
\cline { 2 - 4 } & $\mathbf{x}$ & $\mathbf{y}$ & $\mathbf{z}$ & & \\
\hline $\mathrm{O}(1)$ & 0.0000 & 0.3107 & 0.1184 & 5.56 & 1.0000 \\
$\mathrm{O}(2)$ & 0.1805 & 0.1805 & 0.1805 & 6.79 & 1.0000 \\
$\mathrm{O}(3)$ & 0.2500 & 0.0000 & 0.5000 & 6.20 & 1.0000 \\
$\mathrm{D}(4)$ & 0.2318 & 0.2318 & 0.2318 & 2.83 & $0.4690^{*}$ \\
$\mathrm{D}(5)$ & 0.0000 & 0.4322 & 0.2003 & 3.40 & 0.4680 \\
$\mathrm{D}(6)$ & 0.0000 & 0.3775 & 0.1589 & 5.78 & 0.4690 \\
$\mathrm{D}(7)$ & 0.0000 & 0.3214 & 0.0337 & 4.60 & 0.4690 \\
$\mathrm{D}(8)$ & 0.0677 & 0.2619 & 0.1315 & 4.90 & 0.4680 \\
$\mathrm{D}(9)$ & 0.1260 & 0.2341 & 0.1644 & 8.80 & 0.4680 \\
$\mathrm{H}(10)$ & 0.0279 & 0.0446 & 0.0725 & 11.02 & 0.1086 \\
$\mathrm{C}(11)$ & 0.0000 & 0.0000 & 0.0000 & 10.02 & 0.6464 \\
$\mathrm{C}(12)$ & 0.2500 & 0.5000 & 0.0000 & 24.95 & 0.9342 \\
$\mathrm{H}(13)$ & 0.3016 & 0.5516 & 0.0516 & 27.45 & 0.267 \\
$\mathrm{H}(14)$ & 0.3394 & 0.5000 & 0.0000 & 27.45 & 0.267 \\
$\mathrm{H}(15)$ & 0.2500 & 0.5896 & 0.0000 & 27.40 & 0.267 \\
\hline \hline
\end{tabular}

Table 1. Model of deuterated methane gas hydrate used to process the corresponding kinetic neutron diffraction data.

\footnotetext{
* The values for all D atoms should be the same and were given mistakenly different in Klapproth, 2002.
} 


\section{Appendix A.4}

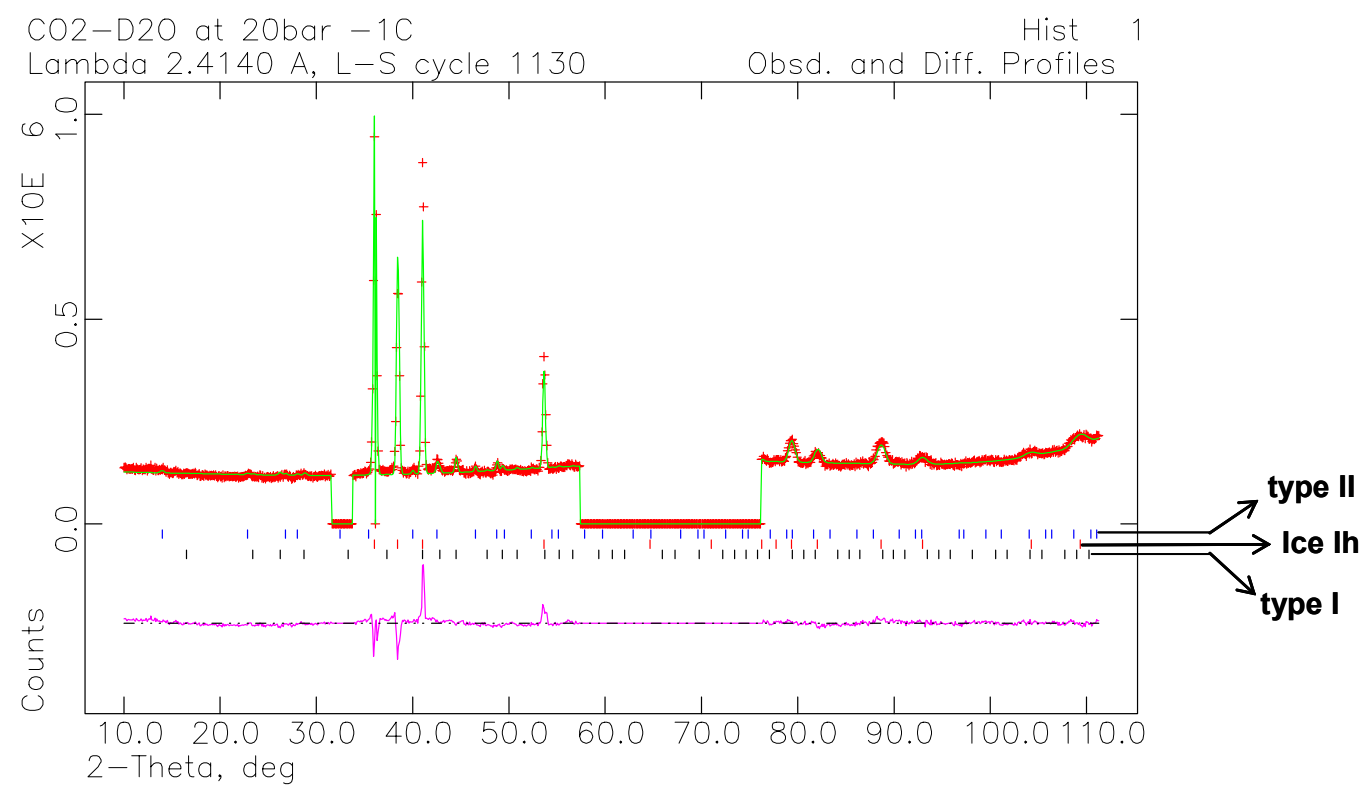

a

Mouse (keyboard): Left(H) - Height, Right(W) - Location Both $(X)$ - exit

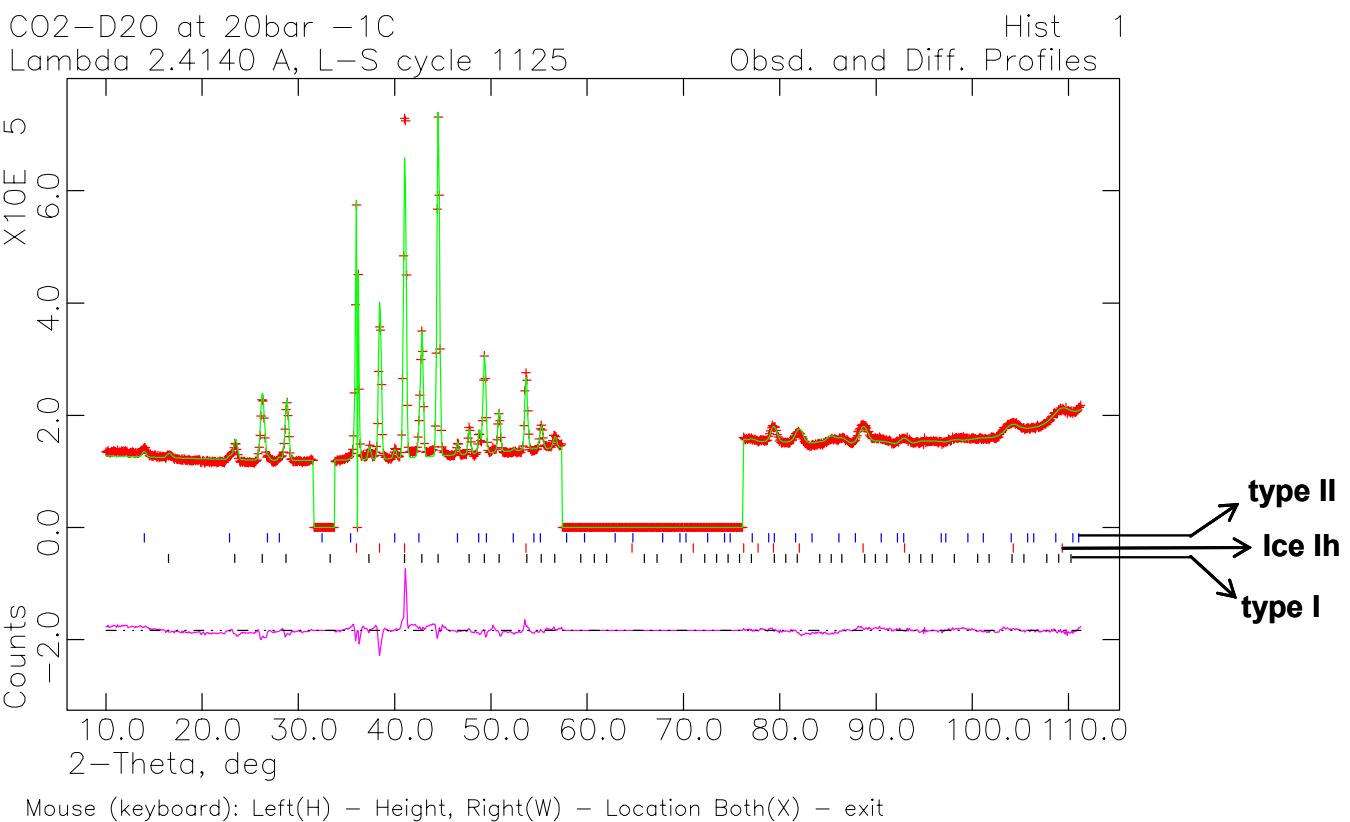

Figure 3. GSAS Rietveld refinement of the data of $\mathrm{CO}_{2}-\mathrm{D}_{2} \mathrm{O}$ hydrate formed at $2.0 \mathrm{MPa}, 272 \mathrm{~K}$ with included type II phase. The diffraction patterns correspond to (a) $5 \mathrm{~min}$ of reaction (type I: (5.1 \pm 0.6$) \%$; type II: (4.4 \pm $0.5) \%)$; and (b) $18 \mathrm{~h}$ of reaction (type I: $(60.4 \pm 0.3) \%$; type II: $(5.6 \pm 0.3) \%)$. The observed and calculated fit values are marked with crosses and a continuous line, respectively. The excluded regions cover the pressure cell scattering. A narrow region (36.0-36.2) $2 \theta$ is excluded because of a dead (not counting) detecting cell. 
Model of type $\mathrm{I}_{2} \mathrm{O}-\mathrm{CO}_{2}$ gas hydrate at $2.0 \mathrm{MPa}$ and $273 \mathrm{~K}$ obtained from neutron diffraction studies (Klapproth, 2002).

\begin{tabular}{cccccc}
\hline \hline \multirow{2}{*}{ Type } & \multicolumn{2}{c}{ Fractional coordinates } & U $\begin{array}{c}\text { Uisox100, } \\
{\left[\AA^{2}\right]}\end{array}$ & Occ. \\
\cline { 2 - 4 } & $\mathbf{x}$ & $\mathbf{y}$ & $\mathbf{z}$ & & \\
\hline $\mathrm{O}(1)$ & 0.0000 & 0.3089 & 0.1182 & 6.48 & 1.0000 \\
$\mathrm{O}(2)$ & 0.1804 & 0.1804 & 0.1804 & 6.60 & 1.0000 \\
$\mathrm{O}(3)$ & 0.2500 & 0.0000 & 0.5000 & 8.88 & 1.0000 \\
$\mathrm{D}(4)$ & 0.2303 & 0.2303 & 0.2303 & 5.02 & 0.4900 \\
$\mathrm{D}(5)$ & 0.0000 & 0.4349 & 0.2016 & 6.78 & 0.4900 \\
$\mathrm{D}(6)$ & 0.0000 & 0.3778 & 0.1629 & 8.70 & 0.4900 \\
$\mathrm{D}(7)$ & 0.0000 & 0.3186 & 0.0376 & 6.13 & 0.4900 \\
$\mathrm{D}(8)$ & 0.0691 & 0.2636 & 0.1355 & 5.61 & 0.4900 \\
$\mathrm{D}(9)$ & 0.1168 & 0.2289 & 0.1607 & 6.86 & 0.4900 \\
$\mathrm{O}(10)$ & 0.4710 & 0.9094 & 0.7545 & 21.10 & 0.2430 \\
$\mathrm{C}(11)$ & 0.2574 & 0.5000 & 0.0000 & 18.76 & 0.4850 \\
$\mathrm{C}(12)$ & 0.0000 & 0.0000 & 0.0000 & 5.20 & 0.5150 \\
$\mathrm{O}(13)$ & 0.0966 & 0.0000 & 0.0000 & 5.20 & 0.1710 \\
\hline \hline
\end{tabular}

Table 2. Model of type I $\mathrm{CO}_{2}$-gas hydrate used to process the corresponding kinetic neutron diffraction data. 
Model of type II $\mathrm{D}_{2} \mathrm{O}-\mathrm{CO}_{2}$ gas hydrate made from a model of type II $\mathrm{N}_{2}$-hydrate (Chazallon, 1999) with $\mathrm{N}_{2}$ replaced by $\mathrm{CO}_{2}$ molecules in the cages. The orientations of the guest molecules are kept with $\mathrm{C}$-atom positioned in the middle between the two $\mathrm{N}$-atoms. The $\mathrm{O}$-atoms are placed according to their distance (1.15 $\AA$ ) from the carbon atom (Klapproth, 2002).

\begin{tabular}{|c|c|c|c|c|c|}
\hline \multirow[t]{2}{*}{ Type } & \multicolumn{3}{|c|}{ Fractional coordinates } & \multirow{2}{*}{$\begin{array}{c}\mathbf{U}_{\text {iso }} \mathbf{x} 100 \\
{\left[\AA^{2}\right]}\end{array}$} & \multirow[t]{2}{*}{ Occ. } \\
\hline & $\mathbf{x}$ & $\mathbf{y}$ & $\mathbf{Z}$ & & \\
\hline $\mathrm{O}(1)$ & 0.8750 & 0.8750 & 0.8750 & 5.00 & 1.0000 \\
\hline $\mathrm{O}(2)$ & 0.7820 & 0.7820 & 0.7820 & 5.00 & 1.0000 \\
\hline $\mathrm{O}(3)$ & 0.8177 & 0.8177 & 0.6296 & 5.00 & 1.0000 \\
\hline $\mathrm{D}(4)$ & 0.8435 & 0.8435 & 0.8435 & 5.50 & 0.4960 \\
\hline $\mathrm{D}(5)$ & 0.8145 & 0.8145 & 0.8145 & 5.50 & 0.4960 \\
\hline $\mathrm{D}(6)$ & 0.7954 & 0.7954 & 0.7266 & 5.50 & 0.4960 \\
\hline $\mathrm{D}(7)$ & 0.8038 & 0.8038 & 0.6805 & 5.50 & 0.4960 \\
\hline $\mathrm{D}(8)$ & 0.8583 & 0.8583 & 0.6279 & 5.50 & 0.4960 \\
\hline $\mathrm{D}(9)$ & 0.7278 & 0.8549 & 0.5867 & 5.50 & 0.4960 \\
\hline $\mathrm{C}(10)$ & 0.0000 & 0.0000 & 0.0000 & 7.76 & 0.9150 \\
\hline $\mathrm{O}(11)$ & 0.0000 & 0.0000 & 0.0665 & 7.76 & 0.3050 \\
\hline $\mathrm{C}(12)$ & 0.3750 & 0.3750 & 0.3750 & 23.23 & 1.0000 \\
\hline $\mathrm{O}(13)$ & 0.3750 & 0.3750 & 0.4415 & 23.23 & 0.3330 \\
\hline
\end{tabular}

Table 3. Model of type II $\mathrm{CO}_{2}$-gas hydrate used to process the corresponding kinetic neutron diffraction data. 


\section{Appendix A.5}

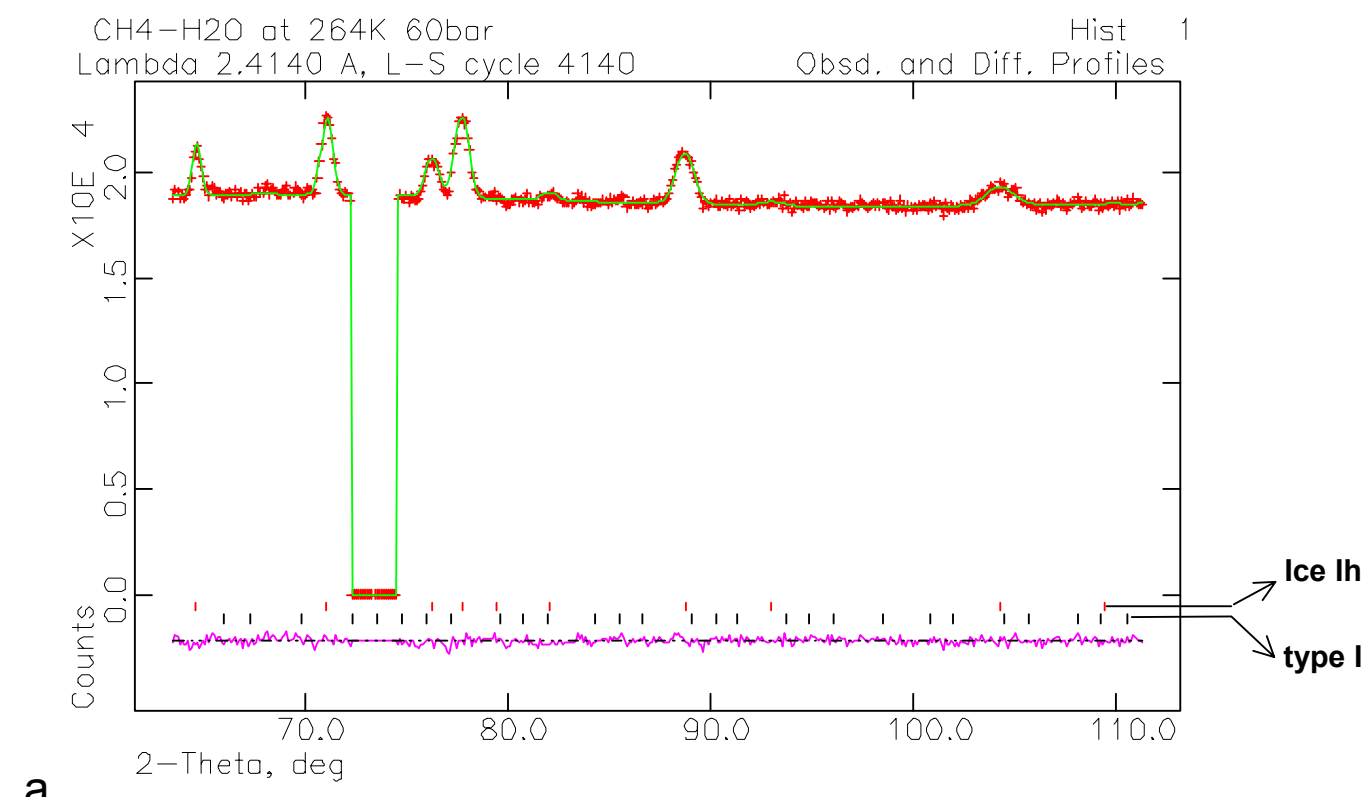

a

Mouse (keyboord) Left(H) - Height, Right(W) - Location Both(X)

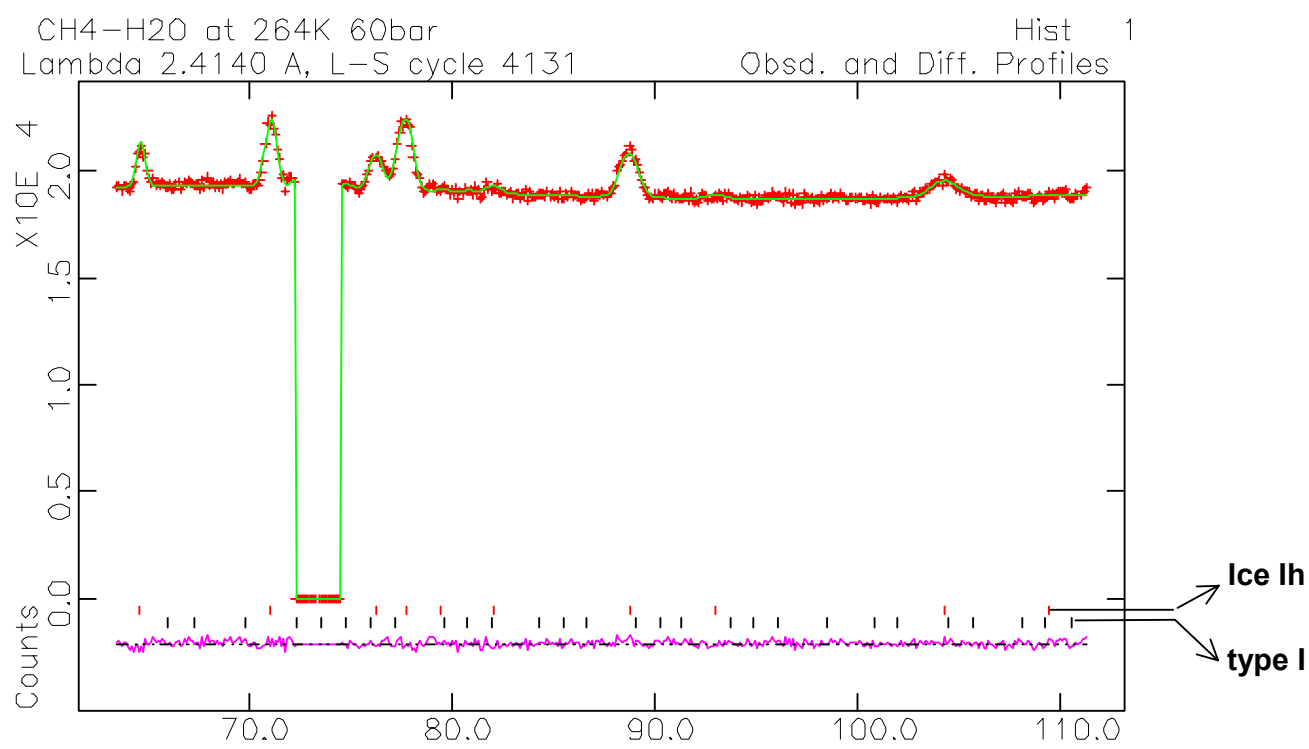

b

Mouse (keyboard). Left $(H)$ - Height, Right $(W)$ - Locotion Both $(X)$ - exit

Figure 4. GSAS plot of the Rietveld fit of the neutron diffraction data related to 10 min slice of $\mathrm{CH}_{4}-\mathrm{H}_{2} \mathrm{O}$ hydrate formation at 6.0 MPa, 264K after (a) $10 \mathrm{~min}$ of the reaction (type-I: $(0.1 \pm 2.0) \%$ ) and (b) $17.5 \mathrm{~h}$ of the reaction (type-I: $(11.8 \pm 1.6) \%)$. Observed and calculated fit values are marked with crosses and a continuous line, respectively. The excluded region covers the pressure cell scattering. 
Model of type $\mathrm{I} \mathrm{H}_{2} \mathrm{O}-\mathrm{CH}_{4}$ gas hydrate was made using the one of $\mathrm{D}_{2} \mathrm{O}-\mathrm{CH}_{4}$-gas hydrate phase ${ }^{1}$.

\begin{tabular}{|c|c|c|c|c|c|}
\hline \multirow[t]{2}{*}{ Type } & \multicolumn{3}{|c|}{ Fractional coordinates } & \multirow{2}{*}{$\begin{array}{c}\mathbf{U}_{\text {iso }} \mathbf{x} 100 \\
{\left[\AA^{2}\right]}\end{array}$} & \multirow[t]{2}{*}{ Occ. } \\
\hline & $\mathbf{x}$ & $\mathbf{y}$ & $\mathbf{z}$ & & \\
\hline $\mathrm{O}(1)$ & 0.0000 & 0.3107 & 0.1184 & 5.56 & 1.0000 \\
\hline $\mathrm{O}(2)$ & 0.1805 & 0.1805 & 0.1805 & 6.79 & 1.0000 \\
\hline $\mathrm{O}(3)$ & 0.2500 & 0.0000 & 0.5000 & 6.20 & 1.0000 \\
\hline $\mathrm{H}(4)$ & 0.2318 & 0.2318 & 0.2318 & 2.83 & $0.4690^{2}$ \\
\hline $\mathrm{H}(5)$ & 0.0000 & 0.4322 & 0.2003 & 3.40 & 0.4680 \\
\hline $\mathrm{H}(6)$ & 0.0000 & 0.3775 & 0.1589 & 5.78 & 0.4690 \\
\hline $\mathrm{H}(7)$ & 0.0000 & 0.3214 & 0.0337 & 4.60 & 0.4690 \\
\hline $\mathrm{H}(8)$ & 0.0677 & 0.2619 & 0.1315 & 4.90 & 0.4680 \\
\hline $\mathrm{H}(9)$ & 0.1260 & 0.2341 & 0.1644 & 8.80 & 0.4680 \\
\hline $\mathrm{H}(10)$ & 0.0279 & 0.0446 & 0.0725 & 11.02 & 0.1086 \\
\hline $\mathrm{C}(11)$ & 0.0000 & 0.0000 & 0.0000 & 10.02 & 0.6464 \\
\hline $\mathrm{C}(12)$ & 0.2500 & 0.5000 & 0.0000 & 24.95 & 0.9342 \\
\hline $\mathrm{H}(13)$ & 0.3016 & 0.5516 & 0.0516 & 27.45 & 0.267 \\
\hline $\mathrm{H}(14)$ & 0.3394 & 0.5000 & 0.0000 & 27.45 & 0.267 \\
\hline $\mathrm{H}(15)$ & 0.2500 & 0.5896 & 0.0000 & 27.40 & 0.267 \\
\hline
\end{tabular}

Table 4. Model of $\mathrm{H}_{2} \mathrm{O}-\mathrm{CH}_{4}$ hydrate used to process the corresponding kinetic neutron diffraction data.

\footnotetext{
${ }^{1}$ A slightly higher $\mathrm{U}_{\text {iso }}$ can be expected for $\mathrm{H}$ as compared to $\mathrm{D}$ atoms. The difference will, however, barely affect the low-angle data of interest in the kinetic diffraction runs and is ignored in this work.

${ }^{2}$ The values for $\mathrm{H}(4)-\mathrm{H}(9)$ atoms should be the same (the corresponding values were given mistakenly different in Klapproth, 2002).
} 


\section{Appendix B}

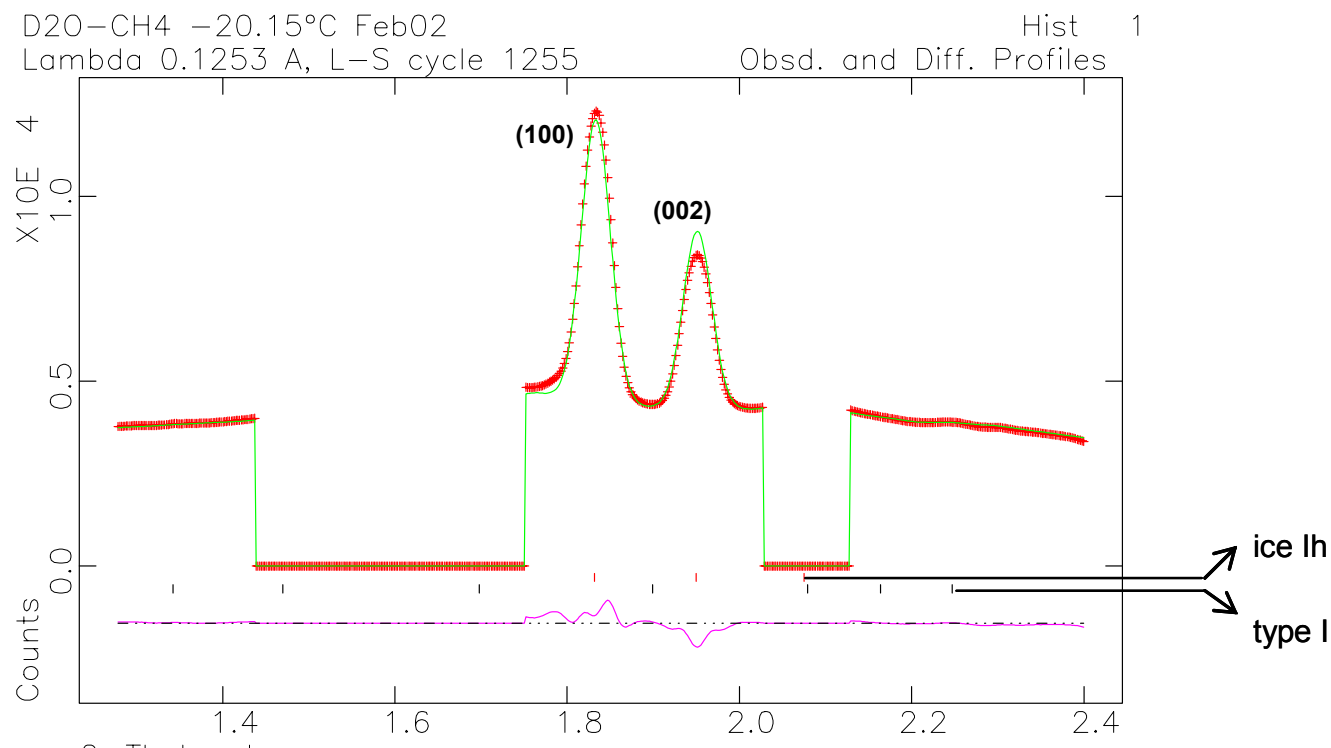

a

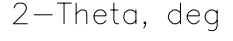

Nouse (keyboard): Left(H) - Height, Right(W) - Location Both(X) - exit

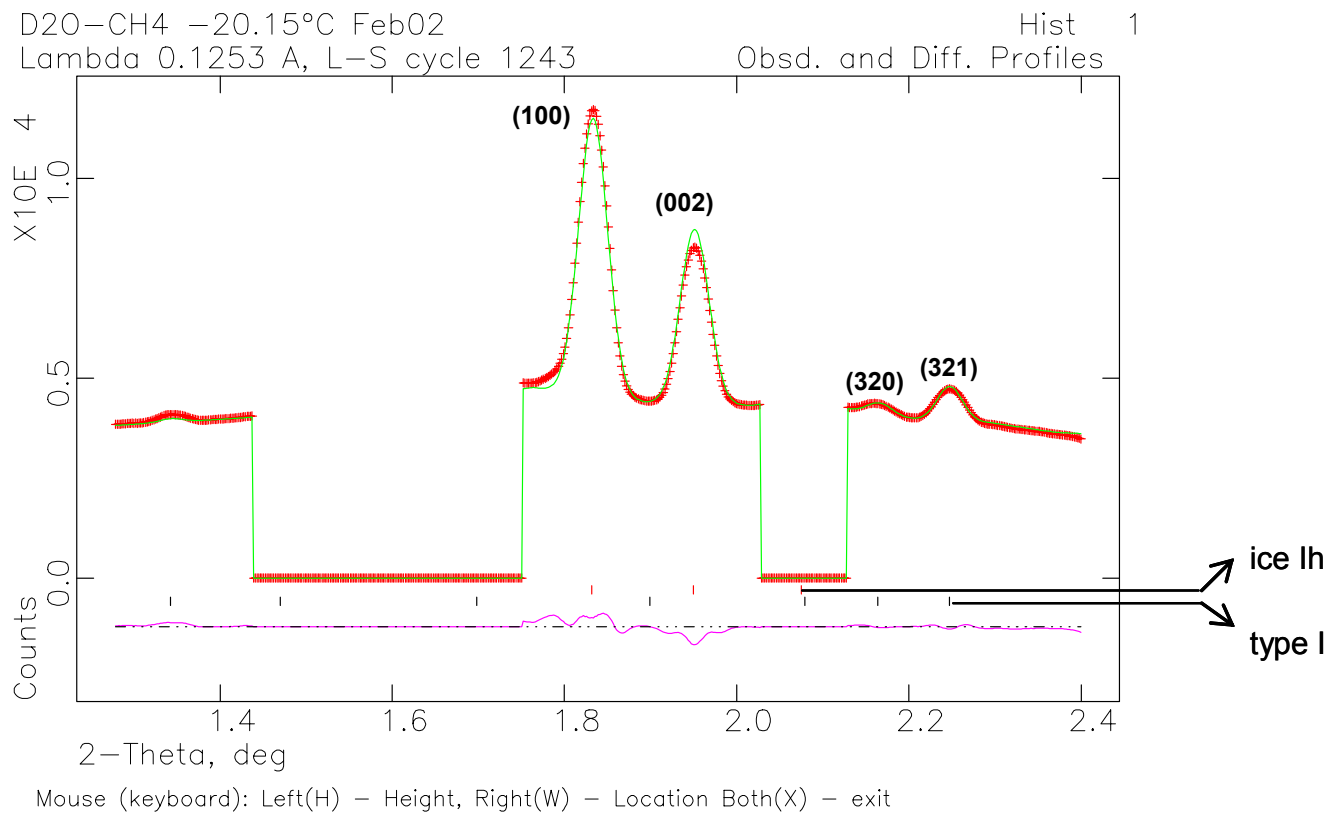

Figure 5. GSAS plot of Rietveld fit of the $\mathrm{X}$-ray diffraction data corresponding to $\mathrm{CH}_{4}-\mathrm{D}_{2} \mathrm{O}$ hydrate formation at 6.0 MPa, $253 \mathrm{~K}$ for (a) $6 \mathrm{~min}$ and (b) for $24 \mathrm{~h}$ of reaction (type I: $(8.0 \pm 0.3) \%$ ). The observed and calculated values are given by crosses and as a continuous line, respectively. The numbers in brackets marked (hkl) Miller indices of the observed Bragg reflections. 2 $\theta$-ranges covering the scattering contributions of the pressure cell or containing overlapping reflections are excluded. The ice was not a good powder and the refinement of the overlapped ice-clathrate peak could affect the value of clathrate fraction as a result of parameters correlations (for details see $\mathrm{Ch}$. 3.2.2). 
Parameters of methane clathrate model used to process X-ray diffraction data. The model is obtained from X-ray diffraction studies (Klapproth, 2002) of $\mathrm{H}_{2} \mathrm{O}-\mathrm{CH}_{4}$ gas hydrate formed at 6.0MPa and $271 \mathrm{~K}$.

\begin{tabular}{|c|c|c|c|c|c|}
\hline \multirow[t]{2}{*}{ Atoms } & \multicolumn{3}{|c|}{ Fractional coordinates } & \multirow{2}{*}{$\begin{array}{c}\mathbf{U}_{\text {iso }} \mathbf{x} 100 \\
{\left[\AA^{2}\right]}\end{array}$} & \multirow[t]{2}{*}{ Occ. } \\
\hline & $\mathbf{x}$ & $\mathbf{y}$ & $\mathbf{z}$ & & \\
\hline $\mathrm{O}(1)$ & 0.0000 & 0.3086 & 0.1173 & 4.00 & 1.000 \\
\hline $\mathrm{O}(2)$ & 0.1845 & 0.1845 & 0.1845 & 4.00 & 1.000 \\
\hline $\mathrm{O}(3)$ & 0.2500 & 0.0000 & 0.5000 & 4.00 & 1.000 \\
\hline $\mathrm{D}(4)$ & 0.2120 & 0.2120 & 0.2120 & 6.46 & 0.500 \\
\hline $\mathrm{D}(5)$ & 0.2950 & 0.0710 & 0.5000 & 6.46 & 0.500 \\
\hline $\mathrm{D}(6)$ & 0.0000 & 0.3550 & 0.1620 & 6.46 & 0.500 \\
\hline $\mathrm{D}(7)$ & 0.0000 & 0.3480 & 0.0460 & 6.46 & 0.500 \\
\hline $\mathrm{D}(8)$ & 0.0300 & 0.2670 & 0.1090 & 6.46 & 0.500 \\
\hline $\mathrm{D}(9)$ & 0.2040 & 0.1470 & 0.1510 & 6.46 & 0.500 \\
\hline $\mathrm{H}(10)$ & 0.0110 & 0.0319 & 0.0518 & 11.02 & 0.121 \\
\hline $\mathrm{C}(11)$ & 0.0000 & 0.0000 & 0.0000 & 10.02 & 0.734 \\
\hline $\mathrm{C}(12)$ & 0.2500 & 0.5000 & 0.0000 & 24.95 & 0.980 \\
\hline $\mathrm{H}(13)$ & 0.2869 & 0.5369 & 0.0369 & 27.45 & 0.280 \\
\hline $\mathrm{H}(14)$ & 0.3138 & 0.5000 & 0.0000 & 27.45 & 0.280 \\
\hline $\mathrm{H}(15)$ & 0.2500 & 0.5640 & 0.0000 & 27.40 & 0.280 \\
\hline
\end{tabular}




\section{Appendix C}

The pressure values measured in the laboratory studies of $\mathrm{CH}_{4}$-clathrate formation were corrected with respect to ambient temperature fluctuations measured at one place, close to the pressure gauge. Gas pressure $P_{0}$, measured at a reference moment $t_{0}$, is related to the ambient temperature $T_{0}^{a i r}$ at this moment. Fluctuations $\widetilde{P}_{i}$ of the measured pressure $P_{i}$ caused by fluctuations of the ambient temperature $T_{i}^{a i r}$ at moments $t_{i} \neq t_{0}$ can be calculated by the law of Charles (see notation in C.1-C.2). At each moment of one running gas hydrate reaction, $P_{i}$ can be regarded as a sum of two values: one, $\bar{P}_{i}$ corresponding to a constant (reference) temperature and another, $\widetilde{P}_{i}$ giving the deviation caused by the change of the temperature with respect to the reference one. After the subtraction of the fluctuations from the measured pressure, values corresponding to constant ambient conditions $\bar{P}_{i}$ can be obtained. A coefficient $\gamma$ is introduced as a multiplication factor giving the pressure corrections for the whole stick (see discussion in the text).

$$
\begin{aligned}
& \mid \begin{array}{l}
P\left(t_{0}\right)=P_{0} \\
P\left(t_{i} \neq t_{0}\right)=P_{i} \\
T^{\text {air }}\left(t_{0}\right)=T_{0}^{\text {air }} \\
T^{\text {air }}\left(t_{i} \neq t_{0}\right)=T_{i}^{\text {air }}
\end{array} \\
& \mid \begin{array}{l}
P_{0}=\bar{P}_{0} \\
P_{i}=\bar{P}_{i}+\gamma \widetilde{P}_{i} \\
\widetilde{P}_{i}=\frac{P_{0}}{T_{0}^{a i r}} T_{i}^{\text {air }} \quad(\mathrm{V}=\text { const }) \\
\bar{P}_{i}=P_{i}-\gamma \widetilde{P}_{i}
\end{array}
\end{aligned}
$$




\section{Appendix D}

It was assumed that on average the initial fissure in a reference ice grain of radius $r_{0}$ had the form of a cleft penetrating to the sphere centre with the small angle $2 \beta$ between its sides $S_{f}$ (see Fig.2, Ch. 4). In general the crack filling was regarded as passing through two analogous stages of (1) hydrate film coating the fissure surface and (2) reaction-controlled growth of the hydrate layer on the two crack sides. Thus, per unit of time, additional fraction $\omega_{S}^{\prime} e^{-\omega_{S}^{\prime} t}$ of the fissure surface was covered with the initial hydrate film, formed of the ice layer of thickness $\delta_{0}{ }^{\prime}$, and on the coated area exposed to the ambient gas, the ice layer of $\omega_{R}^{\prime} / \rho_{i}$ thickness transformed to hydrate. In each case the newly formed porous hydrate layer was $1+E$ times thicker than the consumed ice. The incremental excess hydrate volume entered into the cleft from both sides and led to an increase in the average height $h$ of the fissure filling. The above process was described by the equation:

$$
\beta \frac{d h}{d t}=E \delta_{0}^{\prime} \omega_{S}^{\prime} e^{-\omega_{S}^{\prime} t}+E \frac{\omega_{R}^{\prime}}{\rho_{i}}\left(1-e^{-\omega_{S}^{\prime} t}\right)
$$

and resulted in Eq. (4), Ch.4. Simultaneously, the cleavage-side area (initially equal to $\pi r_{0}^{2} / 2$ ) decreased

$$
\frac{d S_{f}}{d h}=-2 \sqrt{r_{0}^{2}-h^{2}}
$$

whereas the hydrate volume $v_{f}$ formed in and around the fissure increased

$$
\frac{d v_{f}}{d h}=2 \beta S_{f} \frac{1+E}{E}
$$

Sequential integration of the latter equations with respect to $h$ from 0 to $h$ yielded

$$
S_{f}=\frac{\pi r_{0}^{2}}{2}\left(1-\frac{2}{\pi} \arcsin \frac{h}{r_{0}}\right)-h \sqrt{r_{0}^{2}-h^{2}}
$$

and $v_{f}=2 \beta r_{0}^{3} \frac{1+E}{E}\left[\frac{\pi}{2} \xi-\xi \arcsin \xi-\left(1-\xi^{2}\right)^{1 / 2}+\frac{1}{3}\left(1-\xi^{2}\right)^{3 / 2}+\frac{2}{3}\right], \xi=h / r_{0}$.

The total hydrate volume which completely filled the fissure corresponded to $\xi=1$, and the last relation determined the current filling degree $\chi=v_{f} / v_{f}(\xi=1)$ given by Eq. (5), Ch.4. 


\section{Appendix E}

The experimental grain-size fractional histogram of the starting material (ice powder) was approximated by a lognormal distribution:

$$
\varphi_{0}\left(r_{0}\right)=\frac{1}{r_{0} \sigma_{a} \sqrt{2 \pi}} \exp \left[-\frac{\left(\ln r_{0}-a\right)^{2}}{2 \sigma_{a}^{2}}\right],
$$

where $r_{0}$ was the initial ice particle radius; $a$ and $\sigma_{a}^{2}$ are the parameters of the distribution, the mathematical expectation and standard deviation of the random value $\ln r_{0}$, respectively.

The sample was approximated with a truncated form of the above presentation, which was non-zero only within a certain finite interval of $\ln r_{0}$. A $2.5 \%$-accuracy range of $\ln r_{0}$-variation for the lognormal law (E.1) was fixed with the limits $a \pm 2.25 \sigma_{a}$. This means that the initial grainsize distribution function was taken as

$$
\begin{gathered}
f_{0}\left(r_{0}\right)= \begin{cases}1.025 \varphi_{0}\left(r_{0}\right), & r_{0} \in\left[r_{\text {min }}, r_{\text {max }}\right] ; \\
0, & r_{0} \notin\left[r_{\text {min }}, r_{\text {max }}\right] .\end{cases} \\
(\text { E. } 2) \\
r_{\text {min }}=\exp \left(a-2.25 \sigma_{a}\right), \quad r_{\text {max }}=\exp \left(a+2.25 \sigma_{a}\right) .
\end{gathered}
$$

Statistical ensemble of particles in the sample at any moment $t$ was represented by the icecore size distribution function

$$
f\left(r_{i}, t\right)=f_{0}\left(r_{0}\right)\left[\frac{d r_{i}(t)}{d r_{0}}\right]^{-1}, r_{i}>0
$$

Eq. (E.3) was the principal balance equation, which described the evolution of the sample.

By definition, the total fraction of ice grains, which currently were not completely converted to hydrates, was given by

$$
n=\int f\left(r_{i}, t\right) d r_{i}
$$

whereas the ensemble averaged value $\langle\psi\rangle$ of any characteristic $\psi\left(r_{0}, t\right)$, was determined as

$$
\langle\psi\rangle=\int \psi\left(r_{0}, t\right) f_{0}\left(r_{0}\right) d r_{0}
$$

Accordingly, the initial and current mean-volume radii $\left(\bar{r}_{0}\right.$ and $\left.\bar{r}_{i}\right)$ of ice cores and their specific surface area $S_{i}$ were

$$
\bar{r}_{0}=\left\langle r_{0}^{3}\right\rangle^{1 / 3} ; \bar{r}_{i}=\left\langle r_{i}^{3}\right\rangle^{1 / 3} ; S_{i}=3\left\langle r_{i}^{2}\right\rangle /\left(\rho_{i} \bar{r}_{0}^{3}\right) ; \alpha=1-\bar{r}_{i}{ }^{3} / \bar{r}_{0}{ }^{3}
$$


where $\rho_{i}$ was the molar density of ice. Expression for $S_{i}$ should be additionally divided by the factor $1+\bar{\sigma}_{0}{ }^{2}$, where $\bar{\sigma}_{0}$ is the relative standard deviation of the grain size.

A model addition describing the crack filling $\chi$ (see Appendix D) calculates the average filling degree as:

$$
\bar{\chi}=\left\langle r_{0}^{3} \chi / \bar{r}_{0}^{3}\right.
$$

and the reaction degree as:

$$
\alpha=\frac{1}{1-\varepsilon_{f}}\left[\left(1-\frac{\bar{r}_{i}^{3}}{\bar{r}_{0}^{3}}\right)\left(1-\varepsilon_{f} \frac{1+E}{E}\right)+\bar{\chi} \frac{\varepsilon_{f}}{E}\right]
$$

where $\varepsilon_{f}$ is the total crack void fraction of ice grains in a sample. 


\section{Appendix F}

In a random dense packing without particle rearrangement, the current number of contacts (coordination number) $Z$ per reference grain plunged into the monodisperse powder of average particles could be expressed after Arzt (1982) as a linear function of the respective hydrate shell radii $r_{h}$ and $\bar{r}_{h}$ :

$$
Z=Z_{0}+C\left(\frac{r_{h}-r_{0}+\bar{r}_{h}-\bar{r}_{0}}{2 \bar{r}_{0}}\right)
$$

Here $Z_{0}$ was the initial coordination number of the reference ice grain, and $C \sim 15.5$ was the slope of the random density function in the average monosize particle structure. It was assumed that in a polydispersed system $Z_{0}$ monotonically would increase with the relative size of the particle, and a possible parametric approximation could be written as

$$
Z_{0}=Z_{\text {min }}+\left(\bar{Z}_{0}-Z_{\text {min }}\right)\left(r_{0} / \bar{r}_{0}\right)^{\gamma}
$$

where $\bar{Z}_{0} \sim 7$ was the coordination number in the random dense packing of monosize spheres, exponent $\gamma \sim 1-2$ and minimum coordination number $Z_{\min } \sim 3$ are the approximation parameters.

The principal geometric characteristic of the sample structure which determined the interaction of the reference particle with the surrounding medium was the fraction $s$ of the free surface area of the hydrate shell (in units of $4 \pi r_{h}{ }^{2}$ ) exposed to the ambient gas. In the polydisperse powder this quantity was related to the development of a contact area between two growing spheres (reference and average ones) of different external radii $r_{h}$ and $\bar{r}_{h}$. The generalised analogue of Arzt's expression was extended to polydisperse systems (Kuhs and Salamatin, 2003) as

$$
s=1-\frac{\lambda_{0} Z_{0}}{2 r_{h}}\left(r_{h}-r_{0}+\bar{r}_{h}-\bar{r}_{0}\right)-\frac{\lambda_{0} C}{8 \bar{r}_{0} r_{h}}\left(r_{h}-r_{0}+\bar{r}_{h}-\bar{r}_{0}\right)^{2}
$$

where $\lambda_{0}=\bar{r}_{0} /\left(r_{0}+\bar{r}_{0}\right)$.

The mass balance of water molecules directly related the rate of the ice core radius decrease to the rate of the external hydrate radius increase

$$
\frac{d r_{h}}{d t}=-\frac{E r_{i}^{2}}{s r_{h}^{2}} \frac{d r_{i}}{d t}
$$

Correspondingly, from Eqs. (F.3)-(F.4) was obtained for the average grain $\left(r_{0}=\bar{r}_{0}\right.$, $\left.r_{h}=\bar{r}_{h}\right)$ 


$$
\frac{d \bar{r}_{h}}{d t}=-\frac{E \bar{r}_{i}^{2}}{s \bar{r}_{h}^{2}} \frac{d \bar{r}_{i}}{d t}
$$

where the mean free surface fraction $\bar{s}$ was

$$
\bar{s}=1-\frac{\bar{Z}_{0}}{2 \bar{r}_{h}}\left(\bar{r}_{h}-\bar{r}_{0}\right)-\frac{C}{4 \bar{r}_{0} \bar{r}_{h}}\left(\bar{r}_{h}-\bar{r}_{0}\right)^{2} .
$$

For a given reference particle a distance $r$ from the ice core centre to an average contact plane (see Fig. 1, Ch. 4) was introduced as

$$
r=r_{h}\left[1-\frac{2(1-s)}{Z}\right]
$$

Initial macro-porosity of the ice sample $\varepsilon_{m 0}$ was linked to the structural parameters $\bar{Z}_{0}$ and $C$ in (F.1)-(F.2) through quantity $\left(1-\varepsilon_{m 0}\right)^{-1}$ being equal to the maximum relative volume of the average ice hydrate particle with maximum mean particle radius $\bar{r}_{h}$ achievable at $\bar{s}=0$. Current porosity $\varepsilon_{m}$ and specific surface area of the original macro-pore space between the particles $S_{m}$ were formulated as

$$
\varepsilon_{m}=\varepsilon_{m 0}-\alpha\left(1-\varepsilon_{m 0}\right) E, \quad S_{m}=3 \bar{s} r_{h}^{2}\left\langle r_{0}^{2}\right\rangle /\left(\rho_{i} \bar{r}_{0}^{5}\right)
$$




\section{Appendix G}

In order to simulate the process of gas hydrate growth from polydisperse ice powder, POWDER 2 program required two sets of parameters, which described the sample and its reaction. The first one introduced the properties and structural parameters of the ice powder and gas hydrate: molar density of ice $\rho_{i}$; molar density of water in the hydrate $\rho_{h w}$; meso-porosity $\varepsilon_{h}$ of the clathrate phase; powder packing parameters $-\bar{Z}_{0}, C, Z_{\min }$, and $\gamma$. The second one included the reaction parameters of the process: the excess fugacity $F u g=\frac{f-f_{d}}{f_{d}}$; coating rate constant $k_{S}$; reaction rate constant $k_{R}$; permeation coefficient $D$; initial ice-film thickness $\delta_{0}$ converted to hydrate; close-off macro-porosity of the sample $\varepsilon_{m c}$; initial ice-core radius $r_{0}$ and its relative standard deviation Relative STD. The major part of model parameters was considered as known (Table 1):

- parameters of the ice: $\rho_{i}, \varepsilon_{m 0}$ (estimated from the measured packing density of about 65$70 \%$ in the Al-cans)

- parameters of the hydrate: $\rho_{h w}$ (calculated from the known $\mathrm{CH}_{4}-\mathrm{H}_{2} \mathrm{O}$ hydrate structure, Klapproth $2002^{1}$ ), $\varepsilon_{h}\left(\right.$ Suess, $\left.2002^{2}\right)$;

- $\varepsilon_{m c} \sim 0.08$ (Martinerie et al., 1994³)

- $\quad$ parameters of the powder ( $r_{i 0}$ and Relative STD, Ch. 3.4.4); sample packing parameters $\bar{Z}_{0}, C, Z_{\min }, \gamma($ Appendix F);

The parameter of Fug $=\frac{f-f_{d}}{f_{d}}$ is calculated according to the thermodynamic conditions of each reaction.

\footnotetext{
${ }^{1}$ Klapproth, A. (2002). Strukturuntersuchungen an Methan- und Kohlenstoffdioxid-Clathrat-Hydraten. Dissertation, Universität Göttingen

2 Suess, E.; Bohrmann, G.; Rickert, D.; Kuhs, W.F.; Torres, M.E.; Trehu, A.; Linke, P. (2002). Properties and fabric of near-surface methane hydrates at Hydrate Ridge, Cascadia Margin. Proc. 4th Int. Conf. Gas Hydrates, $740-744$.

3 Martinerie P.; Lipenkov V.Y.; Raynaud D.; Chappellaz J.; Barkov N.I., Lorius C. (1994). Air content paleo record in the Vostok ice core (Antarctic): A mixed record of climatic and glaciological parameters. Journal of Geophysical Research, 99 (D5), 10565-10576.
} 


\begin{tabular}{|c|c|}
\hline Parameters and denotations & Values \\
\hline \multicolumn{2}{|l|}{ Ice samples } \\
\hline Ice density $\rho_{i}, \mathrm{kmol} / \mathrm{m}^{3}$ & 51 \\
\hline Initial ice grain radius $r_{0}, \mu \mathrm{m}$ & $25-40$ \\
\hline Relative standard deviation of grain radii & 0.8 \\
\hline Average crack opening angle $\beta, \operatorname{rad} *$ & 0.06 \\
\hline Macro-porosity $\varepsilon_{m 0}$ & 0.33 \\
\hline Coordination number $\mathrm{Z}_{0}$ & 7 \\
\hline Random density slope of particle distribution $C$ & 15.5 \\
\hline \multicolumn{2}{|l|}{ Gas hydrates } \\
\hline Water density in hydrate phase $\rho_{h w}, \mathrm{kmol} / \mathrm{m}^{3}$ & 45 \\
\hline Sub-micron porosity of $\mathrm{CH}_{4^{-}}\left(\mathrm{CO}_{2^{-}}\right)$hydrates $\varepsilon_{h}$ & $0.15(0.1)$ \\
\hline
\end{tabular}

* parameter necessary for the crack-filling model extension (Genov et al., 2004 ${ }^{4}$ )

Table 1. Characteristics of ice samples and gas hydrates.

In order to break the correlations (Eqs.(8),(10), Ch.4) between the left parameters $\delta_{0}, k_{S}, k_{R}$ and $D$, the possible parameters' variations need to be additionally restricted. The parameter $k_{S}$ was estimated as follows: using FE-SEM images of the samples reacted at $263 \mathrm{~K}$ (Ch. 3.4.6) the surface coverage for the most part of the sample lasted between 4 and $8 \mathrm{~h}$. The reaction simulation (see below) at this temperature adjusted the time scale of the surface coating $\left(k_{S}^{-1}\right)$ to $5 \mathrm{~h}$. Additionally, the state observed by FE-SEM of a reacted sample at temperature of $263 \mathrm{~K}$ after about $1 \mathrm{~h}$ was found as a similar to the one reacted at $230 \mathrm{~K}$ for about $11 \mathrm{~h}$ (e.g. Fig 9a, 11 right-down corner, Ch. 3.4). Thus, a ten times longer period for the surface coating stage was assumed at a temperature of $230 \mathrm{~K}$ than at $263 \mathrm{~K}$. A time scale $\left(k_{S}^{-1}\right)$ of $50 \mathrm{~h}$ was estimated initially for methane hydrate formation at a temperature of $230 \mathrm{~K}$. For the rest of the temperatures

${ }^{4}$ Genov, G.; Kuhs, W. F.; Staykova, D. K.; Goreshnik, E.; Salamatin, A. N. (2004). Experimental studies on the formation of porous gas hydrates. American Mineralogist, Vol. 89, No. 8-9, 1228-1239. 
the initial values of $k_{S}$ were assumed (using the estimations above) and adjusted during the fitting procedure (see below).

Thus, only the principal model parameters $\delta_{0}, k_{R}$ and $D$ should be considered as tuning parameters. In particular $\delta_{0}$ controls the stage I, i.e. the increment of the reaction degree within the steepest initial part of the kinetic curve. The final phase of the reaction, i.e. $\alpha>60 \%$, is mainly influenced (limited) by the diffusion coefficient $D$, while the intermediate $10 \%<\alpha<$ $60 \%$, is a transition from reaction-limited to diffusion-limited hydrate formation process where both, $k_{R}$ and $D$, are important.

Model simulations were performed with a manual adjustment of the parameters $k_{S}$ (within a limited range for each temperature), $k_{R}, D$ and $\delta_{0}$ until a best fit between the simulated and experimental data was obtained and a minimum of the discrepancy norm (STD) ${ }^{5}$ was reached. The adjustment of the reaction parameters in the simulations was done as follows: an initial estimation of $k_{S}$ was given and the best-fit values of $k_{R}$ and $\delta_{0}$ were determined within a time period shorted in a way to include stage II at value of $D$ set as sufficiently large $\left(10^{-10}-10^{-9}\right)$. Then $k_{S}$ was adjusted within a time period including only the initial (non-linear) stage I. Then the full data set was reconsidered and $D$ was adjusted to optimize the simulation of diffusion-limited stage III. If a mismatch between the simulated and measured data was observed this procedure was repeated until STD could not be further minimized. It appeared that the model parameters were easily adjusted when the experimental data covered the final stage III of the reaction. In this case, the reaction-limited stage could be distinguished and the typical time $\left(k_{S}^{-1}\right)$ of the hydrate film coating of the ice surface reliably estimated. The values of $\mathrm{F} \sim \frac{D}{k_{R}}$ (Eq.(10)) were constrained of about 0.3 according to the best simulations of long-term reactions. As a result the tuning parameters of each reaction simulation were reproducible within the ranges restricted above.

In some cases, especially at very low temperatures $\mathrm{T}<220-230 \mathrm{~K}$ the initial stage should be additionally sub-divided into two sequential sub-stages simulated by the crack-filling extension of the polydisperse model (POWDER 3).

$5 S T D=\sqrt{\frac{\sum_{i=1}^{N}\left(\alpha_{i}^{s}-\alpha_{i}^{e}\right)^{2}}{N}}$, where $\alpha_{i}^{s}$ and $\alpha_{i}^{e}$ are the simulated and experimental values, respectively. 


\section{Acknowledgements}

I would like to thank Prof. Werner F. Kuhs for the invaluable supervision and help in producing this thesis.

I am also grateful to Alice Klapproth, Georgi Genov, Alexander Schaffler, Viorel Chihaia and Evgeny Goreshnik, for the useful discussions and technical support. Likewise, I thank Stefan Klapp for all these days he spent in front of the computer fighting the statistical distribution of the starting material.

Stefan Adams is gratefully acknowledged for introducing me some of the secrets of GSAS.

I am also thankful to Andrey Salamatin for the discussions on the model.

Thanks to Till Heinrichs (GZG, Abt. Angewandte Geologie) and Kirsten Techmer for teaching me in electron microscopy.

I would like to thank Heiner Bartels, Klaus Haepe and Eberhard Hensel for building the equipment and for software support.

I am grateful to Thomas Hansen, Louis Melesi and Jean-Luc Laborier (ILL,Grenoble) for the help in performing the experiments on D20 and ILL for the beam time and support. Likewise, I am thankful to Helmut Klein and Ulf Garbe (HASYLAB, Hamburg) for the help in performing the experiments on BW5 and HASYLAB for the beam time and support.

This work was supported by the DFG grant Ku920/9. 


\section{Lebenslauf}

Name:

Anschrift:

Geburtstag:

Geburtsort:

Staatsangehörigkeit:

Schulbildung:

Hochschulbildung:
1990

1996

2004
Doroteya Kancheva Staykova

Burg Grona 8

37079 Göttingen

Tel.: 0551/5042580

02.04 .1972

Stara Zagora, Bulgaria

bulgarisch

Mathematische Schule, St. Zagora

Universität Sofia, Physikalische Fakultät

Studienrichtung: Physik

Abschluß: Diplom-Physikerin

Thema der Diplomarbeit: Environmental

Gamma-Ray Spectrometry Utilization for

Determination of Ice and Snow Cover Thickness

Doktorat am Geowissenschaftlichen

Zentrum der Universität Göttingen

Abt. Kristallographie

Thema der Promotion: Kinetic studies of

Methane-Hydrate Formation from Ice Ih

Göttingen, den 23.03.2004 